University of Denver

Digital Commons @ DU

$1-1-2010$

\title{
Controllability and Observability of a Large Scale Thermodynamical System via Connectability Approach
}

Virdiansyah Permana

University of Denver

Follow this and additional works at: https://digitalcommons.du.edu/etd

Part of the Operations Research, Systems Engineering and Industrial Engineering Commons

\section{Recommended Citation}

Permana, Virdiansyah, "Controllability and Observability of a Large Scale Thermodynamical System via Connectability Approach" (2010). Electronic Theses and Dissertations. 506.

https://digitalcommons.du.edu/etd/506

This Dissertation is brought to you for free and open access by the Graduate Studies at Digital Commons @ DU. It has been accepted for inclusion in Electronic Theses and Dissertations by an authorized administrator of Digital Commons@DU. For more information, please contact jennifer.cox@du.edu,dig-commons@du.edu. 
CONTROLLABILITY AND OBSERVABILITY OF

A LARGE SCALE THERMODYNAMICAL SYSTEM

VIA CONNECTABILITY APPROACH

\author{
A Dissertation \\ Presented to \\ the Faculty of Engineering and Computer Science \\ University of Denver
}

In Partial Fulfillment

of the Requirements for the Degree

Doctor of Philosophy

By

Virdiansyah Permana

June 2010

Advisor: Dr. Rahmat Shoureshi 
(C) Copyright by Virdiansyah Permana 2010

All Rights Reserved 
Author: Virdiansyah Permana

Title: CONTROLLABILITY AND OBSERVABILITY OF A LARGE SCALE THERMODYNAMICAL SYSTEM VIA CONNECTABILITY APPROACH Advisor: Dr. Rahmat Shoureshi

Degree Date: June 2010

\begin{abstract}
This study presents a new approach to determine the controllability and observability of a large scale nonlinear dynamic thermal system using graph-theory. The novelty of this method is in adapting graph theory for nonlinear class and establishing a graphic condition that describes the necessary and sufficient terms for a nonlinear class system to be controllable and observable, which equivalents to the analytical method of Lie algebra rank condition. The graph theory of a directed graph (digraph) is utilized to model the system, and the rule of its adaptation in nonlinear class is defined. Subsequently, necessary and sufficient terms to achieve controllability and observability condition are investigated through the structural property of a digraph called connectability. It will be shown that the connectability condition between input and states, as well as output and states of a nonlinear system are equivalent to Lie-algebra rank condition. This approach has been proven to be easier from a computational point of view and is thus found to be useful when dealing with a large system.
\end{abstract}




\section{ACKNOWLEDGEMENT}

The writing of this dissertation has been one of the most significant academic challenges I have ever had to face. The pages of this dissertation hold far more than the culmination of years of study. These pages also reflect the relationship with many generous and inspiring people I have met since the beginning of my graduate work. The list is long and can be longer, but I cherish each contribution to my development as a scholar. It is to them that I owe my deepest gratitude:

To my advisor Dr. Rahmat Shoureshi, gracious mentor whose wisdom, knowledge and guidance, kindness, understanding and patience, as well as commitment to the highest standard, inspired me and motivated me. His mentorship is paramount in providing a well rounded experience consistent with my long-term career goals. He encouraged me to not only grow as good a research engineer but also as an independent thinker. For everything you have done to me, I thank you, Dr. Shoureshi.

To NiSource Energy Technologies and Department of Energy for the technical contributions, experimental data, expertise and financial support. The assistance of Dr. Robert Kramer of NiSource had made this research a challenging, yet enjoyable experience.

To my committee members Dr. Anneliese Andrews, Dr.Corinne Lengsfeld, Dr. Kimon P. Valavanis, Dr. Yun-Bo Yi for their encouraging words, thoughtful criticism, time and attention during busy quarters. 
To my professors at University of Denver as well as other universities, especially: Dr. Roger Salters, Dr. Peter Curtiss, Dr. William Levine, Dr. Moncef Krarti, Dr. David Chichka and Dr. Todd Murphey, who with open heartedly welcomed me for innumerable discussions. Their sincerity to share their knowledge is infectious and has been major driving forces through my graduate career.

To my colleagues for sharing their enthusiasms and comments on my work as well as assisting me with administrative tasks necessary for completing my doctoral program: Donna Kolosky, Dr. Sun Wook Lim, and Soon-To-Be Dr. Christopher Baker.

To my managers: Dr. Kenneth Langer and Jason Hainline, Cindy Cogil, Robert Fagg and John Harriman, as well as Rami Soppa whom I had and have the honor to work with as an engineer. The experience that they shared with me will always be treasured.

To my invaluable network of supportive, forgiving, generous and loving friends without them I could not have survived the process: the Sadagoris, the Renandos, the Arifins, the Ihsans, the Sumarnos, Paskal Kleden, Dida Navayette, Luthfia Tjakraamidjaja, Asri Poeraatmadja and Dhania Iman

To my parents, Sjaiful Bahri and Sri Mulyati, whom I owe everything I am today. They have always supported, encouraged and believed in me, in all my endeavors. To my two sisters, Gita Petrimalia and Riska Andalina, who never had a chance of having a brother nearby. Thank you for the love and understanding during the long years of my education. Also to my parent in law, Sodikin Sastrawidjaja and Ai Komariah, brothers and sister in law whose love is boundless. 
To my two sons, Valdean and Valkean Permana, who both were born before this dissertation is completed and who spent many days being apart to allow me to focus, I am deeply sorry. But I am coming home.

And to my wife Vena Annisa, who stood beside me through thick and thin. Her support, encouragement, patience and unwavering love were undeniably the bedrock upon which the past six years of my life have been built. Her tolerance of my uncontrollable mood is a testament in itself of her unyielding devotion and love.

For my Faith, my Family and my Friends 


\section{TABLE OF CONTENTS}

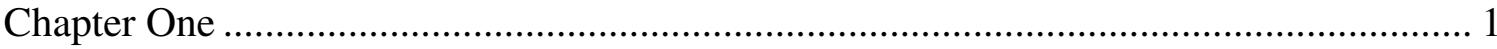

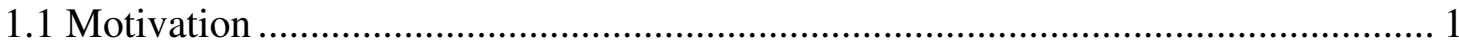

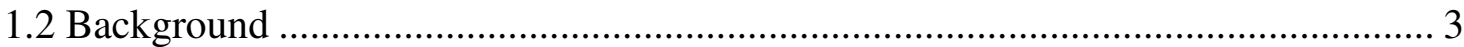

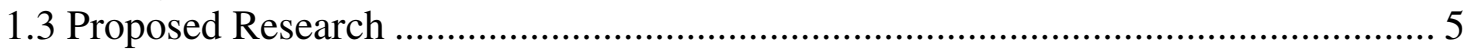

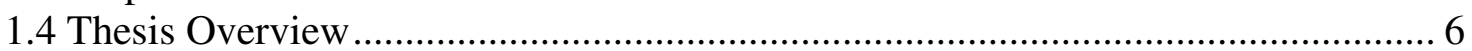

Chapter Two

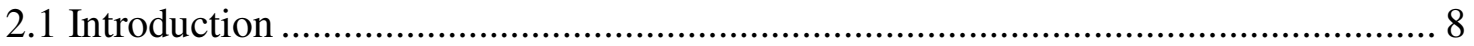

2.2 Controllability and Observability of Linear Systems.............................................. 12

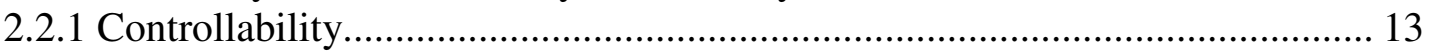

2.2.2 Observability ……………………………………………………….... 14

2.3 Controllability and Observability of Nonlinear System......................................... 15

2.3.1 Controllability of Input-Affine Nonlinear System ............................................ 17

2.3.2 Observability of Input-Affine Nonlinear System ........................................... 20

2.4 Controllability and Observability of Structured Systems ……………………….... 23

2.4.1 Structure Matrices and its Properties............................................................ 24

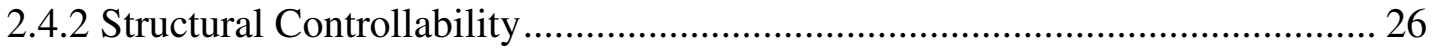

2.4.3 Structural Observability............................................................................... 27

2.5 Controllability and Observability of Large-Scale Systems ....................................... 27

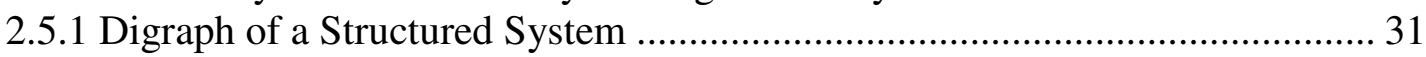

2.5.2 Controllability via Connectability .................................................................... 32

2.5.3 Observability via Connectability .................................................................. 33

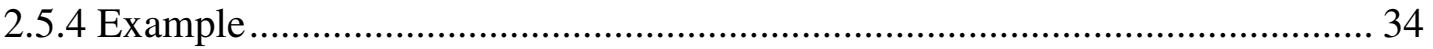

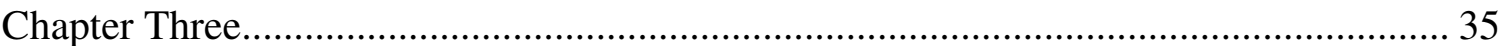

3.1 Thermal Dynamic Model for Control Purposes ....................................................... 36

3.2 Thermal Networks ...................................................................................... 38

3.3 State Space of Thermal Dynamic ................................................................... 43

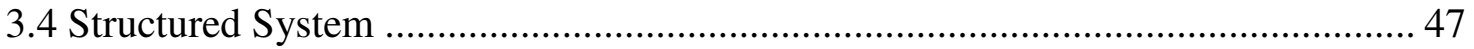

3.5 Digraph of Nonlinear System......................................................................... 50

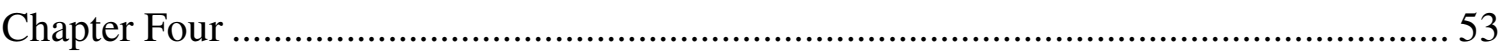

4.1 Building Combined Heat and Power: a System Description ................................... 54

4.2 Nonlinear Thermal Dynamic Model of BCHP System........................................... 64 


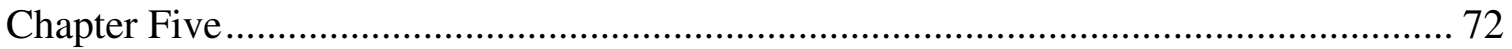

5.1 Controllability of a Non-Linear Thermal Dynamic System.................................. 72

5.2 Controllability of a Structured Non-Linear Thermal Dynamic System .................. 75

5.3 Controllability of a Structured Non-Linear Thermal Dynamic System via

Connectability Approach .............................................................................. 77

5.3.1 Digraph Definition for a Structured Thermal Dynamic System ..................... 78

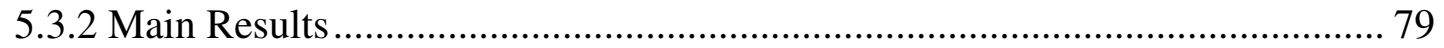

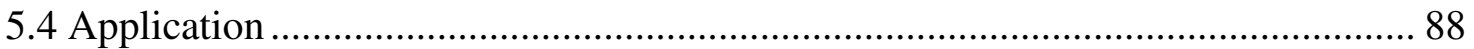

5.4.1 Local Controllability of a nonlinear 2-cell Heat Exchanger ........................... 88

5.4.2 Structural Controllability of BCHP system............................................... 92

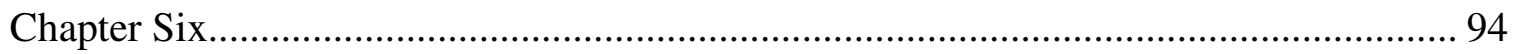

6.1 Observability of Non-Linear Thermal Dynamic System .................................. 94

6.2 Observability of Structured Non-Linear Thermal Dynamic System....................... 97

6.3 Observability of Structured Non-Linear Thermal Dynamic System via

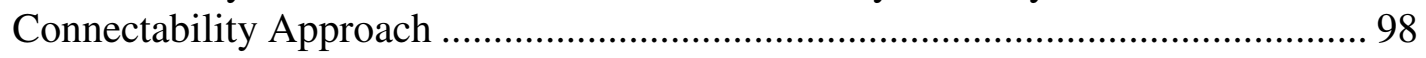

6.3.1 Digraph Definition for Structured Thermal Dynamic System ........................ 98

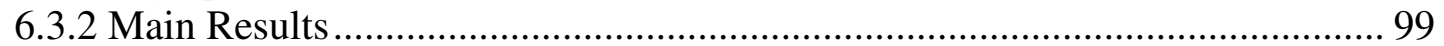

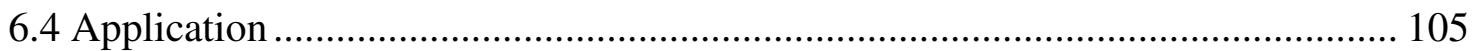

6.4.1 Local Observability of a nonlinear 2-cell Heat Exchanger ........................... 106

6.4.2 Structural Observability of BCHP System ............................................... 108

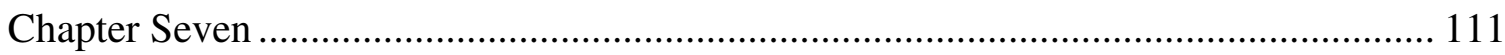

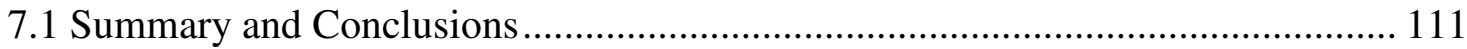

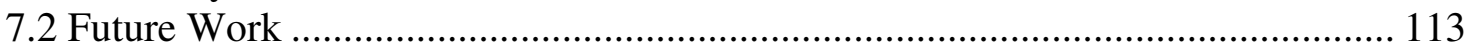

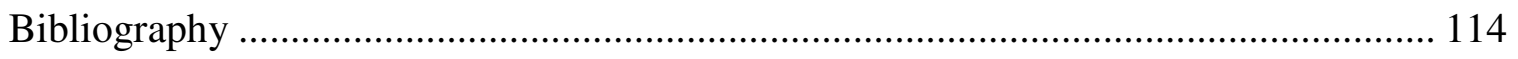

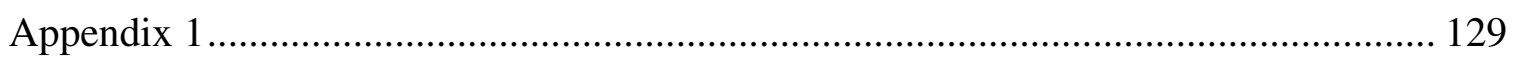

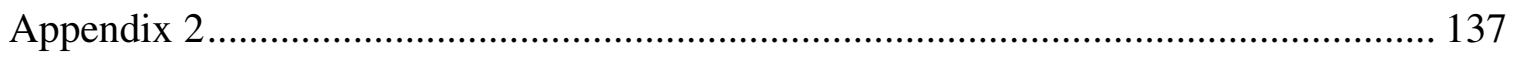

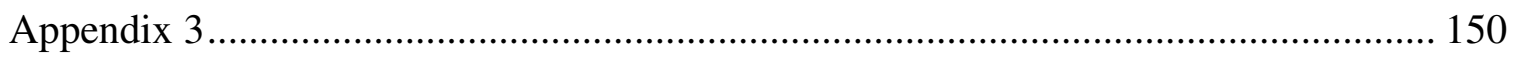

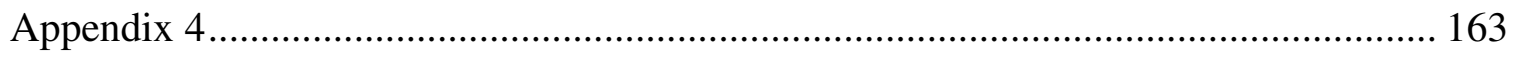




\section{LIST OF FIGURES}

Figure 1: Controllability [Willems 2005, 3] ......................................................... 9

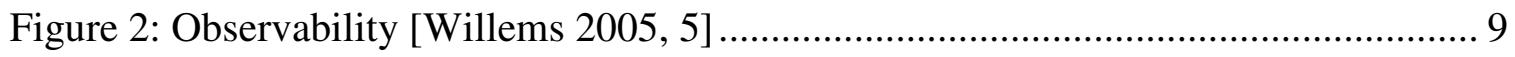

Figure 3: Control System with State Feedback...................................................... 10

Figure 4: State Feedback Control with Observer................................................... 10

Figure 5:(a) Large Scale Systems in Parallel and (b) Large Scale Systems in Series ...... 29

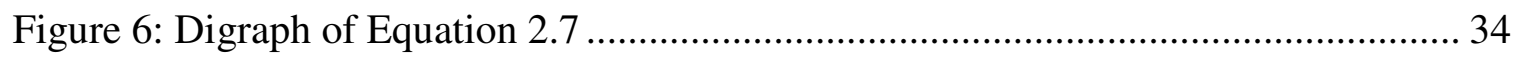

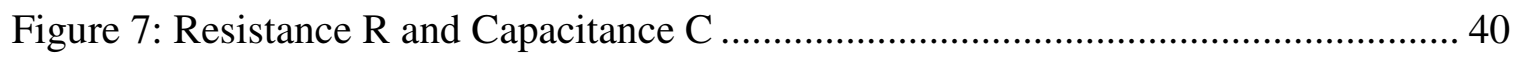

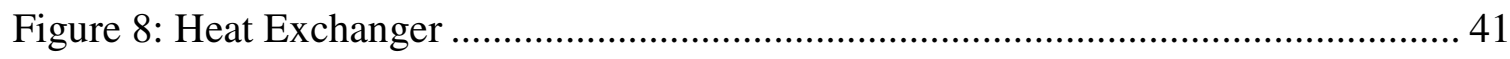

Figure 9: Circuit Model of a Heat Exchanger.......................................................... 42

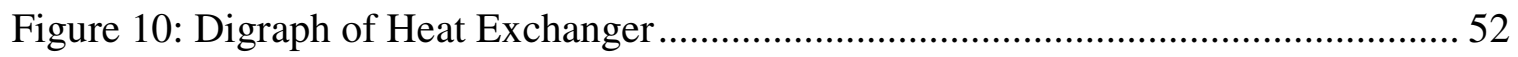

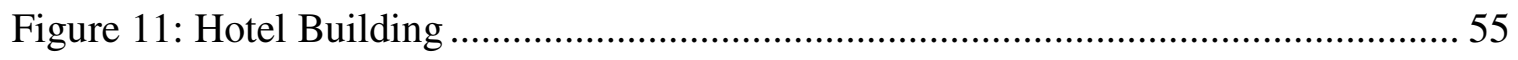

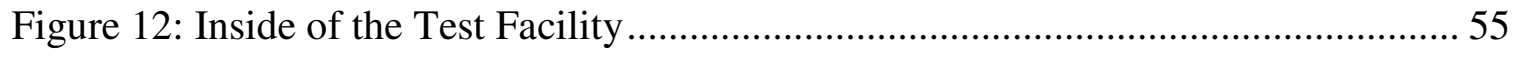

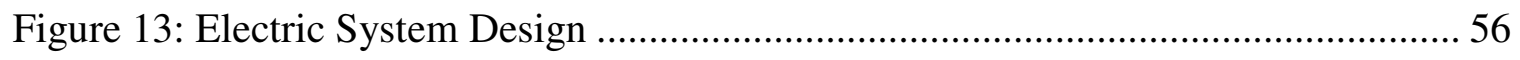

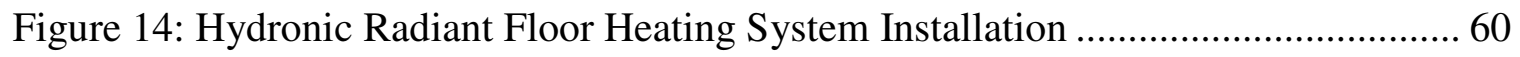

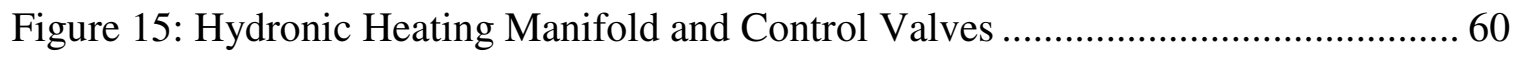

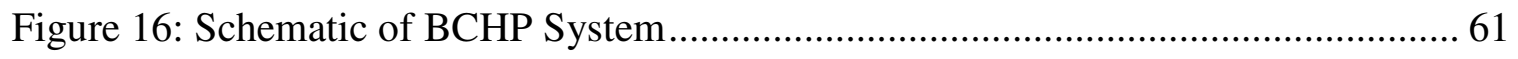

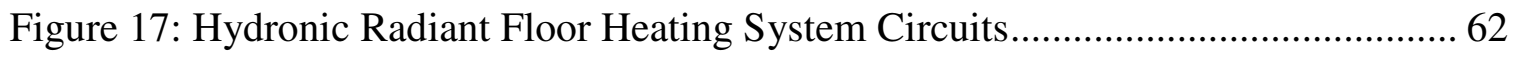

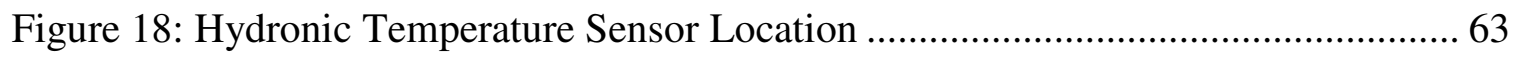

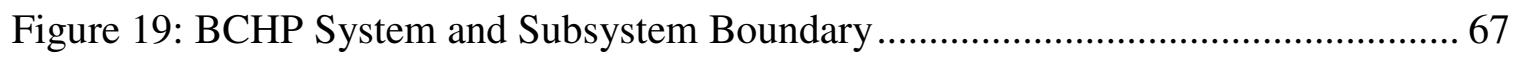

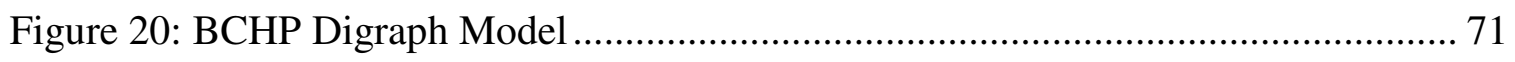




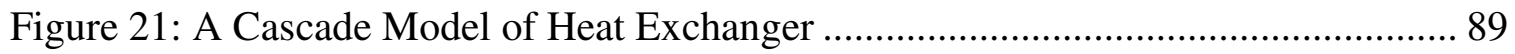

Figure 22: Digraph of 2-Cell Heat Exchanger ......................................................... 91

Figure 23: State Digraph of Nonlinear 2-Cell Heat Exchanger ................................... 91

Figure 24: Input Connectable BCHP State Digraph ................................................ 93

Figure 25: A Cascade Model of Heat Exchanger ...................................................... 106

Figure 26: Digraph of 2-Cell Heat Exchanger ....................................................... 107

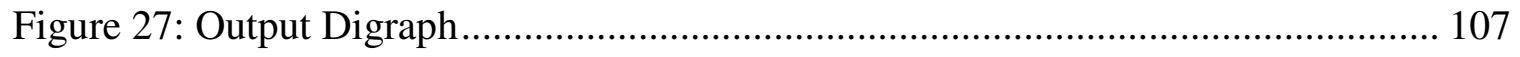

Figure 28: Output Digraph of BCHP system ..................................................... 110

Figure 29: BCHP System and Subsystem Boundary ............................................. 138 


\section{LIST OF TABLES}

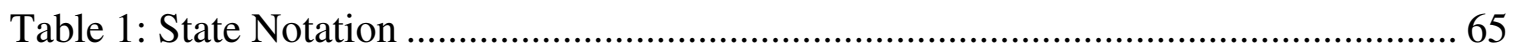

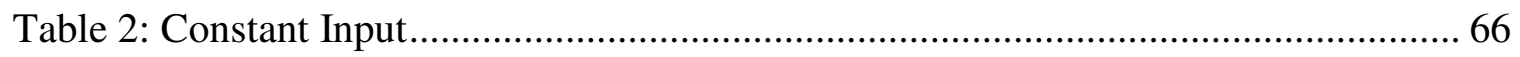

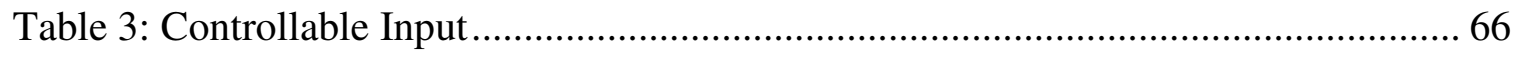

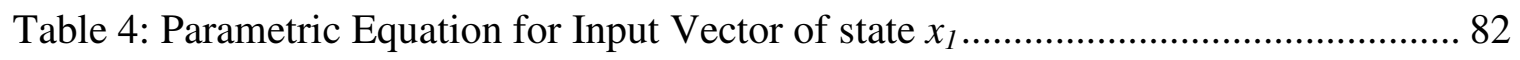

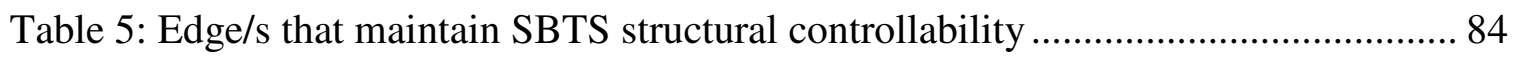

Table 6: Structural Controllability Result Summary ............................................... 86

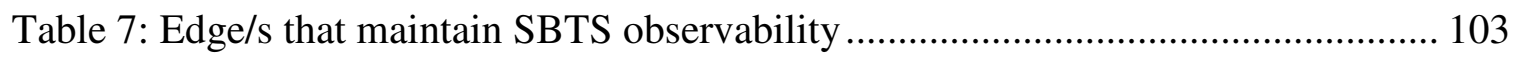

Table 8: Structural Observability Results ............................................................. 104

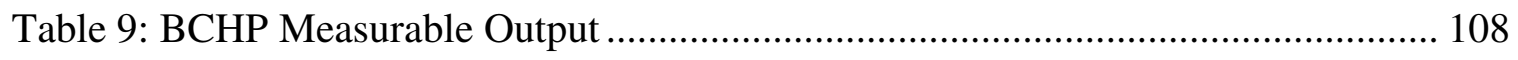

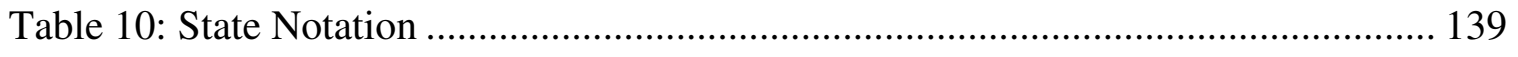

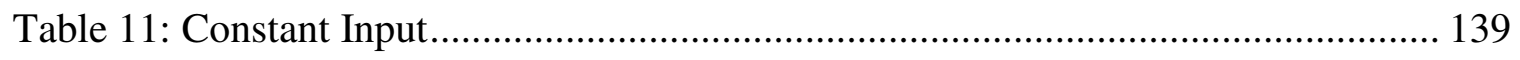

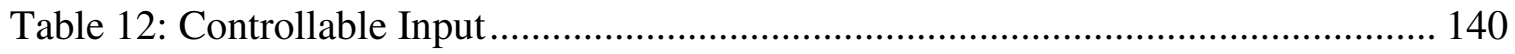




\section{Chapter One}

\section{INTRODUCTION}

This introductory chapter gives the motivation and problem statement for the research, as well as the overview of the thesis.

\subsection{Motivation}

Commercial buildings are a significant and growing consumer of global energy resources. America's 4.9 million commercial buildings span a great variety of functions, sizes, operating schedules and types, from large " $24 / 7$ " hospitals to small retail stores. Providing the necessary energy services in these buildings (lighting, comfort, fresh air, cooking, and power for computers and other equipment) required 6,500 trillion Btu of energy in 2003, $18 \%$ of the USA annual energy use as indicated on Department of Energy's Website. Commercial buildings also constitute the most electric-intensive sector in the country; $55 \%$ of their energy needs are provided by electricity, while $32 \%$ are

provided by natural gas. The growth of energy usage and the energy crisis together with the realization that energy resources are not inexhaustible, and the general trend towards 
a cleaner environment, have led to the development of many practices that aim at using energy as "optimally" as possible. This has materialized in the commercial building sector in the form of building equipment refinements and control system improvements.

In the refinement of building equipment, one of the significant trends, which becomes the interest of this research, is the idea for distributed power generation namely, the notion of power sources near the end user - such as a microturbine, especially with a conjunction of combined heat and power technology (CHP). This technology has received increasing attention by the general public due to its great potential to supply both thermal and electrical energy, which increase its fuel utilization up to $85 \%$ compared to a single electrical only type of distributed power generation as indicated in Department of Energy's Website. Furthermore, CHP also improves power quality and reliability as well as transmission and distribution system support, while at the same time lowering the greenhouse gas production. Since the capital cost of installing such equipment can greatly influence the decision making process, it is desirable to more fully utilize the energy produced. Hydronic radiant floor heating, absorption and desiccant cooling equipment, are examples of thermally activated equipment that can be interconnected with CHP. Furthermore, with deregulation in utility industry, some states also allow the end user to sell their own electricity produced back to them by interconnecting the CHP with the grid.

In control system improvement, application of optimal control has shown the potential to reduce U.S. commercial building energy consumption by about roughly $10 \%$ of current total use [Quartararo, Roth and Brodrick 2006, 66-68]. In addition, control systems offer significant peak demand reduction potential. An optimal control system is 
an emerging system that aims to optimize building energy cost and consumption, while at the same still maintaining the productivity of the building. In a typical office building, for example, to keep the productivity of the occupants in it, its energy use accounts for 30 percent of operating costs which is the largest single category of controllable costs. This condition opens a lot of potential for optimal control to reduce its energy usage while still maintaining the occupants' productivity.

As the building systems to be controlled become more interconnected, it becomes more difficult to design the controller for optimizing system operation. Mathematical model of the system becomes larger and more complex due to the nonlinearity of the real system. These high dimensionalities, nonlinearities, and complexities of interconnection in such a large-scale system provide difficulties not only in modeling, control or optimization, but also in the fundamental issues of stability, controllability, and observability. The problem of assessing these structural properties becomes much more difficult. This thesis is an effort to meet these challenges, especially in the analysis of structural properties of controllability and observability, which becomes the main interest of this research.

\subsection{Background}

Research in the area of controllability and observability of nonlinear systems has been addressed in many works [Lee and Marcus 1961; Hermann 1963; Hermes 1964; Balakrishnan 1966; Mohler and Rink 1968; Haynes and Hermes 1970; Kucera 1970; 
Brockett 1972; Jurdjevic and Sussmann 1972; Kreener 1974; Brockett 1975; Hermann and Krener 1977; Sussmann 1983; Sussmann 1987]. The necessary and sufficient conditions to achieve this property are very well established for several form of nonlinear systems. These conditions have been established using essentially differential geometric approach. However, the use of such tools always assumes the exact knowledge of the state space matrices which characterized the system's model. In many modeling problems, these matrices have a number of fixed zero entries determined by the physical laws while the remaining entries are not known precisely. To study the properties of these systems in spite of the poor knowledge, the idea is to keep the zero/non-zero entries in the state space matrices. Therefore, a model that conserves the fixed zeros while replacing the non-zero entries with a free parameter is considered. There are a huge amount of interesting works in the literature using this type of modeling technique [Lee and Marcus 1967; Lin 1974; Shields and Pearson 1976; Reinschke 1984]. The obtained model is called the structured model. These models are useful to describe the class of systems having the same structure because they capture most of the available structural information from physical laws. Moreover, their study requires a low computational burden which allows one to deal with large-scale systems. Because of these features, the structured systems are adapted to study properties like the controllability and observability. This thesis deals with this kind of system.

Many results on structured systems use graph-theoretic approach. This approach is mainly dedicated to linear systems for which many structural properties such as controllability, observability and stability of several classical control problems have been addressed [Lin 1974; Shields and Pearson 1976; Glover and Silverman 1976; Reinschke 
1984]. Furthermore, from these studies, it follows that graph-theoretic approach provides simple and elegant solutions and so is very well suited to analyze large-scale systems [Gilbert 1963; Chen and Desoer 1970; Brasch and Pearson 1971; Bhandarkar and Fahmy 1972; Grasselli 1972; Ito and Yonemura 1972; Klamka 1972; Hwang and Wolovich 1974; Davison and Wang 1975; Davison 1977]. Unfortunately, the number of studies utilizing graph-theory in nonlinear system is limited. There is clearly a need for the same type of approach for handling nonlinear systems, as well as the large-scale version of it.

\subsection{Proposed Research}

The objective of this research is to develop a simple theoretical analysis for controllability and observability of large-scale nonlinear systems with applications to thermal dynamic systems, which represent a building's energy system considered in this research. More precisely, the proposed research provides a simple necessary and sufficient condition to achieve controllability and observability of a nonlinear system using graph-theoretic approach. Furthermore, it is anticipated that the result of this research is equivalent to the differential geometric based controllability and observability criteria and is suitable to tackle large-scale system.

Most widely used controllability and observability analysis methods require full knowledge of the system to be controlled. Furthermore, these methods are computationally intensive, thus it is appropriate to handle smaller scale type of systems, 
that is, systems in the order of less than ten. However, given today's engineering problem that is characterized with high dimensionalities and nonlinearities, conventional analysis methods are insufficient. Controllability and observability analysis for a large scale linear system has been addressed through the use of structural controllability and obseravability techniques which take advantage of the sparsity structure that a large-scale system carries. However, few researches have addressed this subject for large-scale nonlinear system.

In this research the following subjects are expected to be developed:

1. Develop a graph theoretic approach for nonlinear system structural properties analysis as a way to manage a large-scale version of such class

2. Develop a method to evaluate the necessary and sufficient condition for controllability and observability of a nonlinear system based on the graph theory approach

3. Apply the obtained method to investigate the controllability and observability of real life problems such as building energy systems

\subsection{Thesis Overview}

Chapter two presents a comprehensive review of the area of controllability and observability for linear and nonlinear systems as well as for structured and large-scale systems. Given that the testbed of this research is thermal related energy building systems, Chapter three focuses on the theoretical basis for thermal dynamic system 
models development that is utilized for control design purposes. This includes a digraph model representation for nonlinear systems that is proposed in this research. The modeling theory described in Chapter three is subsequently applied to the actual, real life system under research and the obtained model is presented in Chapter four. The derivation of graph-theoretic approach for analyzing the controllability and observability of a structured nonlinear system is discussed in Chapter five. The derivation includes graph-theoretic definitions that are utilized to satisfy necessary and sufficient conditions for a class of nonlinear systems to be controllable and observable. The proof that the proposed method is equivalent to the Lie algebra rank condition is also presented. Chapter six describes the application of the proposed method to investigate a large-scale structured nonlinear thermal dynamic system that is considered in this research. A summary of the research results, conclusion and future work is presented in Chapter seven. 


\section{Chapter Two}

\section{LITERATURE REVIEW}

To obtain an appreciation for the work related to controllability and observability, it is the goal of the literature review to familiarize the reader with past and present work in the field. Due to the relevance of the main research topic of this dissertation, much of this chapter will focus on past and present success in the area of controllability and observability for systems such as: linear dynamic systems, structured systems, large-scale systems and non-linear systems. These results were selected since they are considered as the groundwork for this research.

\subsection{Introduction}

The two structural properties that play a fundamental role in both the theoretical and practical aspects of control design are controllability and observability (C\&O). Controllability deals with the ability of a dynamic system to steer its state from the initial condition to some desired state by controlling its inputs over a finite amount of time. Observability, on the other hand, deals with the ability of a dynamic system to reconstruct 
or identify the rest of the states' information given partial measurement of the system e.g., inputs and outputs. Figure 1 and 2 illustrate these concepts.
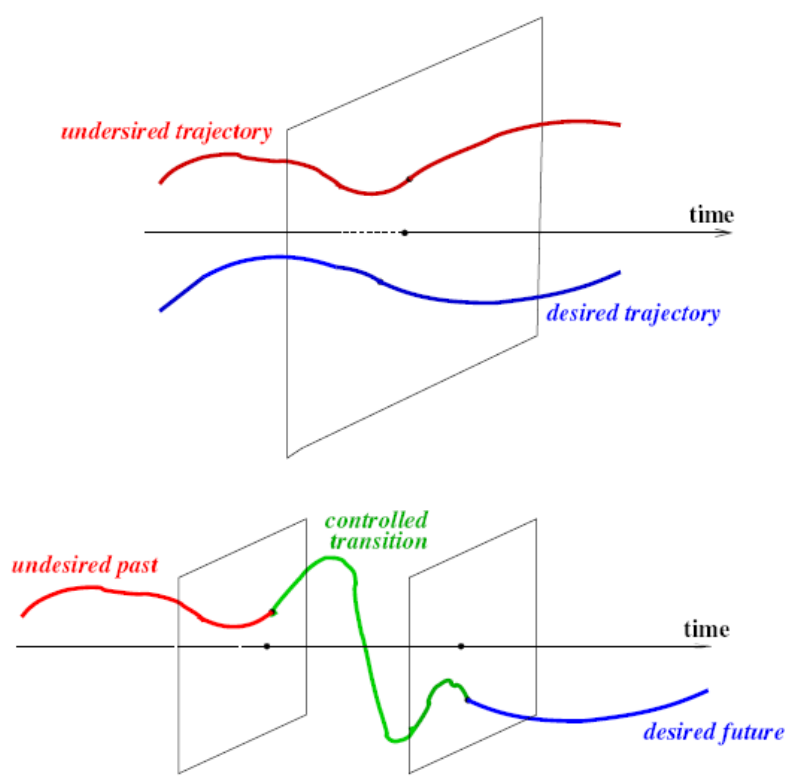

Figure 1: Controllability [Willems 2005, 3]

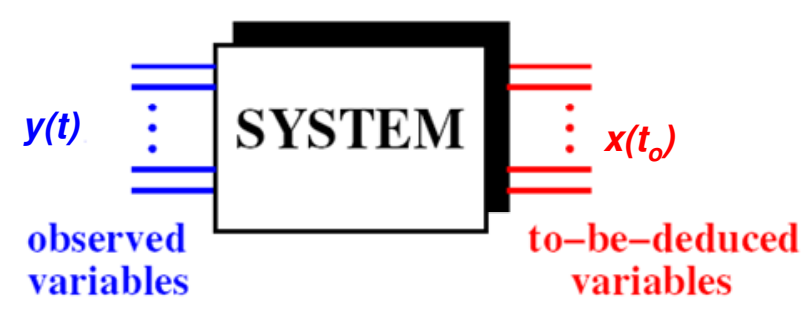

Figure 2: Observability [Willems 2005, 5]

To better understand how the concept of controllability and observability of a system play an important role in control theory, consider the following example. A typical linear dynamic closed-loop system is described by Figures 3 and 4 . 


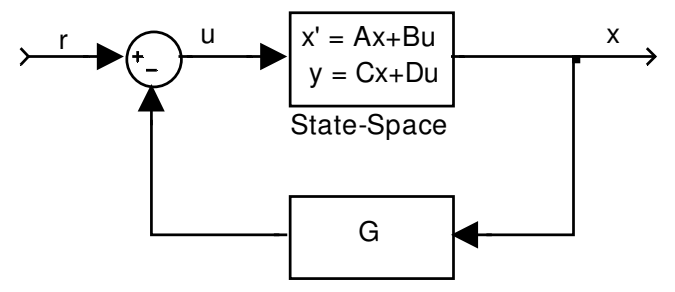

Figure 3: Control System with State Feedback

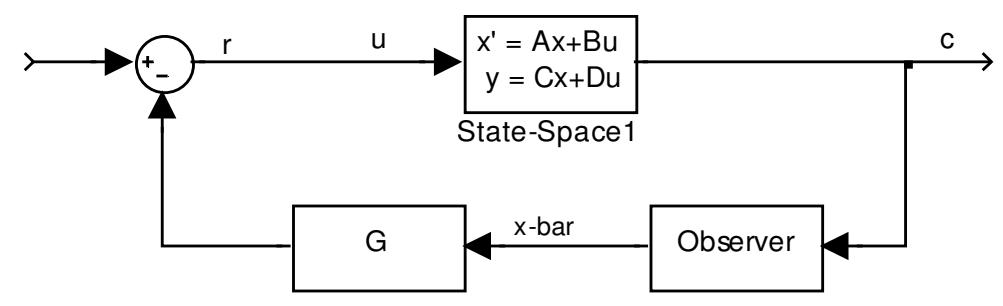

Figure 4: State Feedback Control with Observer

Assume that a state space system in Figure 3 and 4 can be described by Equation

\section{1:}

$\dot{x}(t)=A x(t)+B u(t)$

$y(t)=C x(t)$

A closed-loop system is established by feeding back the state variables $x$ through a constant feedback matrix $G$ which modifies the input function $u(t)$ to the following function:

$u(t)=-G x(t)+r(t)$

The closed loop system of Equation (2.1) is consequently transformed into Equation (2.3) where feedback matrix $G$ is now incorporated:

$\dot{x}(t)=(A-B G) x(t)+B r(t)$ 
The solution to the control system with state feedback is determined by whether or not a feedback matrix $G$ can be derived; that is, if Eigenvalues of $(A-B \cdot G)$ of the closed-loop system are of certain prescribed values. The existence of the solution to this Eigenvalues Technique (also known as Pole Placement Technique), is directly based on the concept of controllability. The system of 2.1 is said to be controllable if there exists a constant feedback matrix $G$ that grants the Eigenvalues of $(A-B \cdot G)$ to be arbitrarily assigned. Furthermore, when the state variable's information is being utilized as the feedback controller, this algorithm requires that the state variables are accessible.

However, feeding back states of the system holds two practical limitations during the design process. First, when the number of state variables for feedback is excessive, the cost of sensing each of these state variables can be unaffordable. Second, the feedback control cannot work as expected when all of the state variables are not directly accessible from the system. Figure 4 shows the block diagram of a closed-loop system, which can overcome this shortcoming through the use of an observer which estimates the system state variable from the system output $c(t)$. The observer estimates the state vector $x$-barbased on the observable output $c(t)$, which subsequently is utilized by the feedback matrix $G$ to generate the control input $c(t)$. When such an observer is attainable from a system described by Equation 2.1, the system is said to be observable. 


\subsection{Controllability and Observability of Linear Systems}

When modern control theory set the stage in the 1950s through the introduction of state-space model concepts, optimal control was born. During the development process of this optimal control, it was recognized that certain non-degeneracy assumptions were needed in establishing the results, that is, under what condition that a dynamic linear system is controllable in its entire state-space? However, it was not until the 1960s that the property of controllability was finally introduced by Kalman [1960, 1], which was applied to characterize the degrees of freedom available when attempting to control a system. Kalman and his team developed a method of testing called controllability rank test that is performed on a certain matrix called the controllability matrix constructed from the state-space matrices of a dynamic linear system. By using this rank condition test, the ability for control input to affect the state vector can be investigated; hence the controllability of a dynamic linear system can be determined. Another topic that was raised during the modern control era is state feedback control, that is, a control system that utilizes system input as a function only of the current state vector. However, in many control situations the system state vector is not available for direct measurement which makes it difficult to evaluate the control input functions. The device which reconstructs this state vector is called an observer which is itself a linear system driven by the available output and inputs to the original system. The ability for the system to obtain an observer is tested by using the observability rank test condition also developed by Kalman [1960, 1] and Luenberger [1964, 74]. The observability rank test condition is performed on a certain matrix formed by the state-space matrices of a dynamic linear 
system, called observability matrix. Both of Kalman's simple rank conditions for controllability and observability are ubiquitous in linear system analysis.

The basic theory of controllability and observability of linear systems developed by Kalman is described as follows. A control system represented by continuous linear time invariant (LTI) systems with the following general form of state-space models is considered:

$\Sigma= \begin{cases}\dot{x}(t)=f(x, u)=A x(t)+B u(t) & ;(\text { State }- \text { Equation }) \\ y(t)=g(x)=C x(t) & ;(\text { Output }- \text { Equation })\end{cases}$

where $x\left(t_{o}\right)=x(0)$ being initial condition; and $x(t) \in \mathfrak{R}^{n}, y(t) \in \mathfrak{R}^{p}, u(t) \in \mathfrak{R}^{l}$ being the state, output, and input vectors of finite dimensional space; and $A \in \Re^{n \times n}$, $B \in \mathfrak{R}^{n \times l}, C \in \mathfrak{R}^{p \times n}$ being time-constant, input and output matrices with constant (timeindependent) elements. The following definition describes the state-space model that represents Equation 1.

\section{Definition 2.2.1 (State Space Representation):}

The state space representation (SSR) that is described by Equation 2.4 is the triplet of constant matrices $(A, B, C)$. The dimension of an SSR is the dimension of the state vector: $\operatorname{dim}[x(t)]=n$. The state space $\chi$ is the set of all states:

$x(t) \in \chi, \operatorname{dim}[\chi]=n$

\subsubsection{Controllability}

Here the state controllability of an LTI system according to Kalman $[1960,1]$ is considered and is defined as the following: 


\section{Definition 2.2.2 (State Controllability):}

A state space model of a system is called state controllable if it is possible to drive any

state $x\left(t_{1}\right)$ to any other state $x\left(t_{2}\right)=x\left(t_{1}\right)$ that is desired with an appropriate input in finite $t=t_{2}-t_{1}$ time

In the case of an LTI system, the above definition is specialized to the following:

\section{Definition 2.2.3 (LTI State Controllability):}

A state space model of an LTI system given by its realization matrices $(A, B, C)$ is called state controllable if it is possible to drive any state $x\left(t_{1}\right)$ to any other state $x\left(t_{2}\right) \neq x\left(t_{1}\right)$ that is desired with an appropriate input in finite time $t=t_{2}-t_{1}$.

Subsequently, in order to determine the controllability of an LTI system, the following theorem defines its necessary and sufficient condition:

\section{Theorem 2.2.1 (Controllability Rank Test):}

A state-space model of an LTI system with realization matrices $(A, B, C)$ is state controllable if and only if the controllability matrix $C_{n}=\left[\begin{array}{lllll}B & A B & A^{2} B & \ldots & A^{n-1} B\end{array}\right]$ is of full rank, that is $\operatorname{rank}\left[C_{n}\right]=n$

\subsubsection{Observability}

Here the state observability of an LTI system according to Kalman $[1960,1]$ is considered and is defined as the following:

\section{Definition 2.2.4 (State Observability):}

Given the inputs and the outputs of a system over a finite time interval, if it is possible to determine the value of the states based on these values and a state-space system model as 
functions of inputs and outputs as well as its derivatives, then the system is called state observable.

In the case of an LTI system, the above definition is specialized to the following:

\section{Definition 2.2.5 (LTI State Observability):}

Given a state-space model by its realization matrices $(A, B, C)$ and the measured input and output signals $\left\{u(t), y(t) \mid t_{o} \leq t \leq t_{f}\right\}$, the system is state observable if the state signal $x$ at a given time $t_{0}$, such that $x\left(t_{0}\right)$ can be determined.

Subsequently, in order to determine the state observability of an LTI system, the following theorem defines its necessary and sufficient condition:

\section{Theorem 2.2.2 (Observability Rank Test):}

Given a state-space model of a LTI system by its realization matrices $(A, B, C)$, this statespace model is state observable if and only if the observability matrix $O_{n}=\left[\begin{array}{lllll}C & C A & C A^{2} & \ldots & C A^{n-1}\end{array}\right]$ is of full rank, that is $\operatorname{rank}\left[O_{n}\right]=n$.

\subsection{Controllability and Observability of Nonlinear System}

The following is the basic theory of nonlinear controllability and observability in a differential geometric approach that were gathered from the works of Lee and Marcus [1961, 36-58], Hermann [1963, 325-332], Hermes [1964, 241-260], Balakrishnan [1966, 465-568], Mohler and Rink [1968, 477-486], Haynes and Hermes [1970, 450-460], Kucera [1970, 160-168], Brockett [1972, 265-284], Jurdjevic and Sussmann [1972, 95-

116], Kreener [1974, 43-52], Brockett [1975, 54-63], Hermann and Krener [1977, 728- 
740], Sussmann [1983a, 1-116; 1987b, 158-194]. Nonlinear finite dimensional systems, which represent a wide class of nonlinear system is considered in this study. The general form of state space models of finite dimension nonlinear system is described by Equation 2.5 .

$\Sigma= \begin{cases}\dot{x}(t)=\tilde{f}(x(t), u(t)) & ;(\text { State }- \text { Equation }) \\ y(t)=\tilde{h}(x(t)) & ;(\text { Output }- \text { Equation })\end{cases}$

where $x, u, y$ being the state, input and output vectors and

$\tilde{f}: \Re^{n} \times \mathfrak{R}^{m} \mapsto \mathfrak{R}^{n}, \tilde{h}: \Re^{n} \times \mathfrak{R}^{m} \mapsto \mathfrak{R}^{p}$ being the smooth nonlinear mappings. If the nonlinear functions $\tilde{f}$ and $\tilde{h}$ above are in a special form, an input-affine form is obtained:

$\Sigma= \begin{cases}\dot{x}(t)=\tilde{f}(x(t), u(t))=g_{0}(x(t))+\sum_{i=1}^{m} g_{i}(x(t)) u_{i}(t) & ;(\text { State }- \text { Equation }) \\ y(t)=\tilde{h}(x(t))=h(x(t)) & ;(\text { Output }- \text { Equation })\end{cases}$

with the same state, input, and output vector $x, u$, and $y$ as above, and with the smooth nonlinear mappings $g_{i}: \mathfrak{R}^{n} \mapsto \mathfrak{R}^{n}$ for $i=0,1, \ldots, m, h: \mathfrak{R}^{n} \mapsto \mathfrak{R}^{p}$. It is important to observe that the input signals enter into the input-affine nonlinear state-space model in a linear way, that is, the mapping $\tilde{f}$ in the original general nonlinear state-space model in Equation 2.5 is linear with respect to $u$.

From the nonlinear control system theory point of view, the state-space model of a nonlinear system $\Sigma$ in Equation 2.6 is considered to represent a nonlinear control system whose system state $x$ evolves on an $n$-dimensional smooth connected manifold $M . x(t) \in M$ is the state of the system at time $t \in \mathfrak{R}$ where $M \subseteq \mathfrak{R}^{n}$ (an open subset of $\left.\Re^{n}\right), u_{1}(t), \ldots u_{m}(t)$ are real valued input functions that are piecewise constant and can take any value in an open interval $\mathrm{I} \subseteq \mathfrak{R}$ containing zero, and $y(t) \in \mathfrak{R}^{p}$ are the 
output vectors. $g_{0}: \mathfrak{R}^{n} \mapsto \mathfrak{R}^{n}$ is the drift vector field (analytic) on $M$, and $g_{i}: \Re^{n} \mapsto \Re^{n}$ for $i=1, \ldots, m$ are control vector fields (analytic) on $M$. It is assumed that the system $\Sigma$ is complete, that is, for every bounded measurable input $u_{i}(t)$ and every $x^{0} \in M$ there exists a solution to $\tilde{f}(x(t), u(t))$ such that $x\left(t^{0}\right)=x^{0}$ and $x(t) \in M$ for all

$t \in \mathfrak{R}$. Notation $\left.\left(u(t), \mid t^{0}, t^{1}\right\rfloor\right)$ is used to denote functions defined on $\left\lfloor t^{0}, t^{1}\right\rfloor$. In response to a set of constant inputs, the state of the system evolves along an integral curve of one of these vector fields. More generally, the state trajectory generated by a piecewise constant input vector will be composed of several segments, each of which lies along the integral curve of one of these vector fields.

Furthermore, the control variable $u$ represents the externally applied control inputs to the systems and the output variable $y$ represents the observable parameters of the system. The state variable $x$ may or may not be directly measurable and is used to represent the memory of the system. The past history of $\Sigma$ affects its future evolution only through information conveyed by this variable. Since the study is geared toward the local rather than global analysis, $U$ is denoted as an open neighborhood of $x^{0} \in M$.

\subsubsection{Controllability of Input-Affine Nonlinear System}

The problem statement of controllability in the nonlinear system case is characterized by the set of states that are reachable from a given initial state [Isidori 1995, 1-99]. The following definitions describe these concepts. 


\section{Definition 2.3.1 ( $U-$ reachable $)$ :}

Given a subset $U \subseteq M, x^{1}$ is $U$-reachablefrom $x^{0}$ (denoted by $\left.x^{1} R_{U} x^{0}\right)$ if there exists a bounded measurable control $\left.\left(u(t), \mid t^{0}, t^{1}\right]\right)$ satisfying $u(t) \in \Omega$ for $t \in\left[t^{0}, t^{1}\right\rfloor$ such that the corresponding solution $\left(x(t),\left[t^{0}, t^{1}\right)\right.$ of differential equation (2.6) satisfies $x\left(t^{0}\right)=x^{0}$, $x\left(t^{1}\right)=x^{1}$ and $x(t) \in U$ for all $t \in\left[t^{0}, t^{1}\right]$. Denote $R\left(x^{0}\right)=\left\{x^{1} \in M: x^{1} R x^{0}\right\}$ the set of points reachable from $x^{0}$

Based on this definition, the state controllability for a nonlinear system in general is defined as follows:

\section{Definition 2.3.2 (State Controllability):}

The system $\Sigma$ is said to be controllable at $x^{0}$ if $R\left(x^{0}\right)=M$ and $\Sigma$ controllable if $R(x)=M$ for every $x \in M$

This definition however may result in a longer time to reach the point near $x^{0}$. Therefore, a stronger notion of controllability is addressed locally which requires that the trajectory stay near $x^{0}$.

\section{Definition 2.3.3 (Local State Controllability):}

The system $\Sigma$ is said to be locally controllable at $x^{0}$ if for every neighborhood $U$ of $x^{0}$, $R_{U}\left(x^{0}\right)$ is also a neighborhood of $x^{0} ; \Sigma$ is locally controllable if it is locally controllable at every $x \in M$

Subsequently, a controllability distribution $\Delta_{C}$ is formulated. The controllability distribution $\Delta_{C}$ is the nonlinear analog of linear controllability matrix that is constructed using the Lie algebra of vector fields $\tilde{f}(x(t), u(t))$ on $M$ corresponding to constant 
control $u \in \mathrm{I}$. Hence, the controllability distribution $\Delta_{C}$ is equivalent to the controllability matrix $C_{n}$ of a linear system. It was suggested that if the dimension of $\Delta_{C}$ is constant or if the system $\Sigma$ is analytic, then there exists a unique maximal submanifold $M^{\prime}$ of $M$ through $x^{0}$ which carries all the trajectories of $\Sigma$ passing through $x^{0}$ such that any point on this submanifold can be reached from $x^{0}$ going forward and backward along the trajectories of the system. In particular if the dimension of $\Delta_{C}\left(x^{0}\right)$ is $n$ then $M^{\prime}=M$. Hence, the system is "controllable" in some sense. The following theorems define the necessary and sufficient conditions for a nonlinear system to be locally controllable.

\section{Theorem 2.3.1 (Controllability Rank Test):}

It is said that $\Sigma$ satisfies the controllability rank condition at $x^{0}$ if in a neighborhood of $x^{0}, \operatorname{dim}\left[\Delta_{C}\right]=n$. If this holds for all $x^{0} \in M$, then $\Sigma$ satisfies the controllability rank condition. Thus, if $\Sigma$ satisfies the controllability rank condition at $x^{0} \in M$, then $\Sigma$ has the local reachability property at $x^{0}$

On the basis of the above explanation, clearly the first step toward the analysis of local controllability of a nonlinear system is to find $R$, which in this case is established through the derivation of a controllability distribution $\Delta_{\mathrm{C}}$ using the Lie bracket. Isidori [1995, 1-99] proposes an algorithm for constructing the controllability distributions as follows: 


\section{Algorithm for Constructing the Controllability Distribution}

1. Starting Point: $\Delta_{0}=\operatorname{span}\left\{g_{1}, \ldots, g_{m}\right\}$

2. Development of the controllability Distribution: $\Delta_{k}=\Delta_{k-1}+\sum_{i=0}^{m}\left[g_{i}, \Delta_{k-1}\right]$

Note that one term in the last sum $\left[g_{i}, \Delta_{k-1}\right]$ is computed by using the functions

$\left(\phi_{1}, \ldots, \phi_{l}\right)$ spanning the distribution $\Delta_{\mathrm{k}-1}:\left[g_{i}, \Delta_{k-1}\right]=\operatorname{span}\left\{\left[g_{1}, \phi_{1}\right], \ldots,\left[g_{l}, \phi_{l}\right]\right\}$

3. Stopping Condition: If $\exists k^{*}$ such that $\Delta_{k}=\Delta_{k-1}$, then $\Delta_{\mathrm{C}}=\Delta_{k^{*}}=\left\langle g_{0}, \ldots, g_{m} \mid \Delta_{0}\right\rangle$

Once the controllability distribution is established, the reachable set rank test condition can be performed.

\subsubsection{Observability of Input-Affine Nonlinear System}

The problem statement of observability in the nonlinear system focuses on finding the condition where the initial state $x^{0}$ can be distinguished given the output measurement [Isidori 1995, 1-99]. Therefore, the definition on distinguishability, or in this case indistinguishability as well as observability, is presented here:

\section{Definition 2.3.4 ( $U$ - indistinguishable ):}

Given a subset $U \subseteq M$, and $x^{0}, x^{1} \in U, x^{0}$ is $U$-indistinguishable from $x^{1}$ (denoted $\left.x^{1} I_{U} x^{0}\right)$ if for every control $\left(u(t),\left[t^{0}, t^{1}\right]\right)$ whose trajectories $\left(x^{0}(t),\left[t^{0}, t^{1}\right]\right)$ and $\left(x^{1}(t),\left[t^{0}, t^{1}\right]\right)$ from $x^{0}$ and $x^{1}$ both lie in $U$, fails to distinguish between $x^{0}$ and $x^{1}$, such that, if $x^{0}(t) \in U$ and $x^{1}(t) \in U$ for $t \in\left[t^{0}, t^{1}\right]$, then $\Sigma_{x^{0}}\left(u(t),\left[t^{0}, t^{1}\right]\right)=\Sigma_{x^{1}}\left(u(t),\left[t^{0}, t^{1}\right]\right)$. Denote $I\left(x^{0}\right)=\left\{x^{1} \in M: x^{1} I x^{0}\right\}$ the set of points indistinguishable from $x^{0}$ 
Based on this definition, the state observability for a nonlinear system in general is defined as follows:

\section{Definition 2.3.5 (State Observability):}

The system $\Sigma$ is said to be observable at $x^{0}$ if $I\left(x^{0}\right)=\left\{x^{0}\right\}$ and $\Sigma$ is observable if $I(x)=\{x\}$ for every $x \in M$

However, this definition may result in a longer time to distinguish a point near $x^{0}$. Therefore, a stronger notion of observability that is addressed locally is introduced.

\section{Definition 2.3.6 (Local State Observability):}

The system $\Sigma$ is said to be locally observable at $x^{0}$ if for every neighborhood $U$ of $x^{0}$, $I_{U}\left(x^{0}\right)=\left\{x^{0}\right\} ; \Sigma$ is locally observable if this is true for every $x \in M$

In the spirit of the approach to nonlinear controllability described previously, an analogous method to the observability for a nonlinear system is developed for nonlinear systems. The relevant object in this study is the observation space $\Omega_{O}$, the smallest linear space of functions on $M$ which contains the observations $g_{1}(x), \ldots, g_{n}(x)$ which are closed with respect to Lie differentiation by vector fields $\tilde{f}(x(t), u(t))$, and the differentials of $\Omega$ denoted by $d \Omega$. It is suggested that if the dimension of $d \Omega_{O}$ is constant over $M$ then indistinguishability exists over $M$. In other words, there exists a system with the same input-output behavior as $\Sigma$, but which is "observable" in the sense that neighboring points are distinguishable. In particular, if the dimension of $d \Omega$ is always $m$ then $\Sigma$ has this property.

The following theorems define the necessary and sufficient condition for a nonlinear system to be locally observable. 


\section{Theorem 2.3.2 (Observability Rank Test):}

$\Sigma$ satisfies the observability rank condition at $x^{0}$ if in a neighborhood of $x^{0}$, $\operatorname{dim}\left[d \Omega_{O}\right]=n$. If this holds for all $x^{0} \in M$, then, $\Sigma$ satisfies the observability rank condition. Thus, if $\Sigma$ satisfies the observability rank condition at $x^{0} \in M$, then $\Sigma$ has the local distinguishability property at $x^{0}$

Based on the above explanation, the first step toward the analysis of local observability of nonlinear systems is to construct the observability co-distribution that is based on Observation space $\Omega$ using the Lie derivative on its output function and vector field. Isidori [1995, 1-99] proposes an algorithm for constructing the observability codistributions $d \Omega$ as follows:

\section{Algorithm for Constructing the Observability Co-Distribution}

1. Starting Point: $\Omega_{0}=\operatorname{span}\left\{d h_{1}, \ldots, d h_{p}\right\}$

2. Development of the observability Distribution: $\Omega_{k}=\Omega_{k-1}+\sum_{i=0}^{m} L_{g_{i}} \Omega_{k-1}$

3. Stopping criterion: if there exists an integer $k^{*}$ such that $\Omega_{k^{*}}=\Omega_{k_{-1}-1}$, then

$$
\Omega_{0}=\Omega_{k^{*}}=\left\langle g_{0}, \ldots, g_{m} \mid \Omega_{0}\right\rangle
$$

Once the observability co-distribution is established, the observability rank test condition can be performed. 


\subsection{Controllability and Observability of Structured Systems}

The controllability and observability in the framework of traditional control theory assumes the exact knowledge of the state space matrices characterizing the system's model. In many modeling problems however, these matrices have a number of fixed zeros entries determined by the physical laws while the remaining are not known precisely. To study the properties of these systems in spite of poor knowledge, the idea is to preserve the zero/non-zero entries in the state space matrices. Thus, models where the fixed zeros are conserved while the non-zeros are replaced by free parameters are considered here. This kind of model is called a structured model and is very useful to describe the class of systems having the same structure. They capture most of the structural information available from physical laws.

Interestingly, the study of structured systems may be considered to have been started with Lin [1974, 201-208], and also in later papers Glover \& Silverman [1976, 534-537] and Shields \& Pearson [1976, 203-212], where they all studied controllability and observability of structured systems. Lin proposed the notion of structural controllability and observability for structured linear systems, where instead of using numerically given matrices $A, B$ and $C$, the corresponding structure matrices $[A],[B]$, and $[C]$ of the same dimensions are considered. Furthermore, the structural rank condition is utilized to test it. This proposed theory is based on Lin's previous theory, which proves that a property of a system holds structurally for a structurally equivalent system if the property under investigation holds numerically for almost admissible numerical realizations. Thus, structural controllability and observability become the process of 
analyzing whether or not at least one admissible realization of a system, with the same structure that is controllable and observable in the usual numerical sense, exists. Furthermore, Lin also introduced a graph theoretic concept, where the structured system is represented by a graph, and structural controllability and observability analysis can now be investigated based on the connectability between the system states with the input and output, respectively. Later on, Glover \& Silverman [1976, 534-537] and Shields \& Pearson [1976, 203-212] extend Lin's study [1974, 201-208] to multi input and multi output systems where both concentrated on an analytic approach.

\subsubsection{Structure Matrices and its Properties}

The following definitions are needed to better understand the concept of a structured system [Lin 1974; Shields and Pearson 1976; Reinschke 1984]. First, given a general matrix $Q$, its structure matrix $[Q]$ is defined as follows:

\section{Definition 2.4.1 (Structured Matrix):}

The elements of a structure matrix $[Q]$ are either fixed at zero or indeterminate values which are assumed to be independent of one another.

$[Q]_{i, j}= \begin{cases}0 & \text { if } \quad w_{i j}=0 \\ V & \text { otherwise }\end{cases}$

Hence, $[Q]$ is the characteristic matrix of the non-zero entries of $Q$.

Furthermore, when the sign of the matrix element is very important to be

maintained e.g. for system property analysis, the signed structure matrix $\{Q\}$ represents the signed structure of a matrix $Q$. 


\section{Definition 2.4.2 (Signed Structured Matrix):}

The elements of a structure matrix $\{Q\}$ are either fixed at zero or indeterminate values which are assumed to be independent of one another.

$$
\{Q\}_{i, j}=\left\{\begin{array}{lll}
0 & \text { if } & w_{i j}=0, \\
+V & \text { if } & w_{i j}>0, \\
-V & \text { if } & w_{i j}<0,
\end{array}\right.
$$

The following definitions describe the basis of a structured system. They are applied to develop structural properties of structured systems.

\section{Definition 2.4.3:}

A numerically given matrix $Q$ is called an admissible numerical realization (with respect to $[Q]$ ) if it can be obtained by fixing all indeterminate entries of $[Q]$ at particular values. Two matrices $Q^{\prime}$ and $Q^{\prime \prime}$ are said to be structurally equivalent if both $Q^{\prime}$ and $Q^{\prime \prime}$ are admissible numerical realizations of the same structure matrix $[Q]$.

\section{Definition 2.4.4:}

A property holds structurally within a class of structurally equivalent systems if the property under investigations holds numerically "for almost" all admissible numerical realizations.

Using the basis of structured system theory defined above, structural properties can now be derived. Structural Rank is one of the structural properties that is essential in deriving the concept of structural controllability and observability.

\section{Definition 2.4.5 (Structural Rank):}

A set of independent entries of $[Q]$ are defined as a set of indeterminate entries, no two of which lie on the same line (row or column). 
The structural rank (for short, s-rank) of $[Q]$ is defined as the maximum number of elements contained in at least one set of independent entries.

It should be noted that the s-rank of $[Q]$ is equal to the maximal rank (in the usual numerical sense) of all admissible numerical matrices $Q$.

\subsubsection{Structural Controllability}

When the concern in the controllability analysis is in the form of structured matrices, structural controllability is applied to ensure the given structured system is controllable [Lin 1974; Shields and Pearson 1976; Glover and Silverman 1976; Reinschke 1984]. Hence, the following definition is applied.

\section{Definition 2.4.6 (Structural Controllability):}

A class of systems given by its structure matrix pair $[A, B]$ is said to be structurally controllable (for short, s-controllable) if there exist at least one admissible realization $(A, B) \in[A, B]$ being controllable in the usual numerical sense

Furthermore, in order to determine if the structured system is structurally controllable, the necessary and sufficient conditions that must be satisfied are described by the following theorem.

\section{Theorem 2.4.1:}

A linear system with structure matrices $([A],[B],[C])$ is:

Structurally controllable, if the block matrix $[A, B]$ is of full structural rank: $s-\operatorname{rank}([A, B])=n$, with $n$ being the number of state variables. 


\subsubsection{Structural Observability}

When the concern in the observability analysis is in the form of structured matrices, structural observability is applied to ensure the given structured system is observable [Lin 1974; Shields and Pearson 1976; Glover and Silverman 1976; Reinschke 1984]. Hence, the following definition is applied.

\section{Definition 2.4.7 (Structural Observability):}

A class of systems given by its structure matrix pair $[A, C]$ is said to be structurally observable (for short, s-observable) if there exists at least one admissible realization $(C, A)^{T} \in[A, C]^{T}$ being observable in the usual numerical sense.

Furthermore, in order to determine if the structured system is structurally controllable, the necessary and sufficient condition that must be satisfied is described by the following theorem.

\section{Theorem 2.4.2:}

A linear system with structure matrices $([A],[B],[C])$ is structurally observable if the block matrix $[C, A]^{T}$ is of full structural rank, that is, $s-\operatorname{rank}\left([C, A]^{T}\right)=n$, with $n$ being the number of state variables.

\subsection{Controllability and Observability of Large-Scale Systems}

Today's engineering problem characterized by a higher degree of complexity and larger numbers of dimensions of its mathematical model has created one of the biggest challenges for control theory in order to come up with a satisfactory control solution 
[Jamshidi 1997, 1]. The well established classical control theory is now insufficient to apply as the computational effort required to model the system, analyze its structural properties and design the controller has become either impossible or uneconomical to solve even with modern computer technology [Siljak 2005, 1]. Therefore it is only natural to seek techniques which reduce the computational effort of these large-scale systems. For this reason, a considerable amount of interest in the research area for largescale dynamic systems began as early as the 1960 [Gilbert 1963, 128-151]. The earliest efforts which focused on reducing the computational effort, were realized by taking advantage of the special structure that a large scale system generally holds, that is, a structured model [Lin 1974; Shields and Pearson 1976; Glover and Silverman 1976; Reinschke 1984]. When a special structure is identified, that is, a large-scale system with its matrice coefficients consists of relatively few nonzero elements, it allows the system to either be kept intact or decoupled into smaller subsystems. When keeping the large structure intact, the system sparseness allows the structure to be transformed into a format where efficient computation can be performed. Such techniques include "compact basis triangularization" and "generalized upper bounding" [Siljak 1999, 209-224]. On the other hand, when decoupling is visible, the original system is divided into a number of subsystems involving a certain adjusted coefficient that represents the interconnection parameter. Hence, the subsystem can be resolved independently and the solution to the overall original system is realized [Brittain, Otaduy, Perez, and Rovere 1988, 108-112]. These techniques are called the multilevel or hierarchical approach and the approach have led the endeavor of exploring different techniques to solve large-scale system problems in modeling, structural property analysis, as well as control design. 


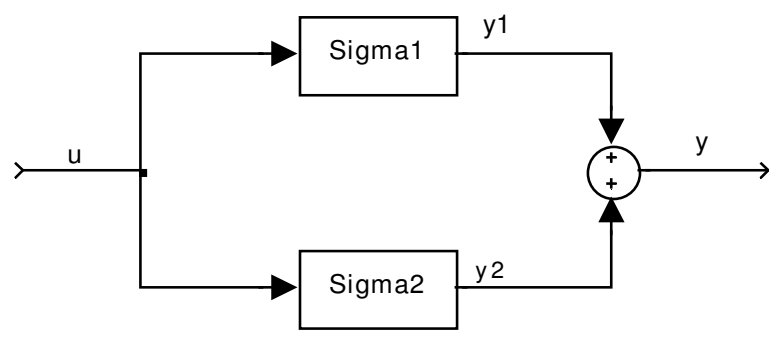

(a)

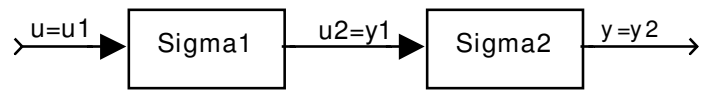

(b)

Figure 5:(a) Large Scale Systems in Parallel and (b) Large Scale Systems in Series

The controllability and observability of large scale systems itself were first considered in 1963 where the classical rank condition method was applied to two different structure combinations of two linear subsystems, namely series and parallel [Gilbert 1963, 128-151]. Assuming that each subsystem has distinct Eigen values and they are both controllable and observable under the rank condition, the result turned out to be different when they formed these two structures. Two subsystems are in series where the output of the first subsystem becomes the input of the other. Under this condition, depending on the parameter of each subsystem, there is the possibility that this combination is uncontrollable and unobservable. On the other hand, when the two subsystems are in parallel that is, when both subsystems share the same input and the overall output is the sum of the two outputs, assuming each subsystem has distinct eigen values, under this condition, the large-scale system will always be controllable and observable as long as each subsystem is also controllable and observable. 
Since this initial work, the interest toward this subject grew. Many studied to explore other techniques that do not rely on the classical rank condition since computing the rank conditions on systems with such large dimensions posed an ill numerical problem. Furthermore, when the tests fail, there is no indication how the rank deficiency can be removed and fixed even in relatively simple situations. For these reasons, system structure in conjunction with graph theory was considered by Lin [1974, 201-208]. Here, the concepts of structural rank, structural controllability and observability, as well as connectability were introduced. The central problem in determining the controllability and observability of a large scale system using the structural properties and graph theory is about finding the connectability between subsystems as well as between system input/output and system state [Chen and Desoer 1970; Brasch and Pearson 1971; Bhandarkar and Fahmy 1972; Grasselli 1972; Ito and Yonemura 1972; Klamka 1972; Hwang and Wolovich 1974; Davison and Wang 1975; Davison 1977].. While the method of finding the controllability and observability $(\mathrm{C} \& \mathrm{O})$ of this large scale linear system can be considered established, little literature has been found which discusses the C\&O for large scale nonlinear systems [Boukhobza and Hamelin 2007, 1968-1974]. However, the approaches that are utilized for the large scale linear systems have inspired and assisted the research to conceptualize the method of checking the $\mathrm{C} \& \mathrm{O}$ of large-scale nonlinear systems. The next few sections describe the concept of controllability and observability using a graph theoretic approach for linear systems that provides a simple and elegant solution and so is very well suited to analyze large-scale system. 


\subsubsection{Digraph of a Structured System}

Graph theory, which first appeared in Euler's paper in 1736 [Hopkins and Wilson 2004, 198-207], is the study of mathematical structures that are utilized to model pairwise relations between objects from a certain collection. A "graph" in this context refers to a collection of nodes or vertices and a collection of edges that connect pairs of vertices [Deo 1974, 1]. A graph may be undirected which means that there is no distinction between the two nodes associated with each edge or directed, where its edges may be directed from one vertex to another. Many physical situations, however, entail directed graphs such as: the street map of a city with one-way streets, flow networks with valves in the pipe, and electrical networks. Structured systems can also be represented elegantly by means of directed graphs or simply called digraphs. Using this type of representation, it is possible to study well-known system theoretic properties from a graph theoretic point of view. For this reason, a digraph is employed to model a large-scale linear system.

The following terminologies are defined to understand the digraphs [Reinschke 1984, 1]. The graph $G=(V, E)$ of a structured LTI system represented by a state space model is defined by a vertex set $V$ and an edge set $E$. The vertex set $V$ is given by $U \cup X \cup Y$ with $U=\left\{u_{1}, \ldots, u_{m}\right\}$ the set of input vertices, $X=\left\{x_{1}, \ldots, x_{n}\right\}$ the set of state vertices, and $Y=\left\{y_{1}, \ldots, y_{p}\right\}$ the set of output vertices. Denoting $\left(v, v^{\prime}\right)$ for a directed edge from the initial vertex $v \in V$ to the terminal vertex $v^{\prime} \in V$, the edge set $E$ is described by $E_{A} \cup E_{B} \cup E_{C}$ with $\quad E_{A}=\left\{\left(x_{j}, x_{i}\right)[A]_{i, j} \neq 0\right\}, \quad E_{B}=\left\{\left(u_{j}, x_{i}\right)\left[[B]_{i, j} \neq 0\right\} \quad\right.$ and $E_{C}=\left\{\left(x_{j}, y_{i}\right)[C]_{i, j} \neq 0\right\}$. In the latter, for instance $[A]_{i, j} \neq 0$ means that the $(i, j)$ th entry of the matrix $[A]$ is a parameter (nonzero). 
In order to perform a structured system properties analysis from a graph theoretic point of view, the following digraph terminologies are useful. Let $W, W^{\prime}$ be two nonempty subsets of the vertex set $V$ of the graph $G$.There exists a path from $W$ to $W^{\prime}$ if there is an integer $k$ and there are vertices $w_{0}, w_{1}, \ldots, w_{k} \in V$ such that $w_{0} \in W$, $w_{k} \in W^{\prime}$ and $\left(w_{i-1}, w_{i}\right) \in E$ for $i=1,2, \ldots, k$. The vertex $w_{0}$ is called the start vertex. and the vertex $w_{k}$ is called the end vertex. The path consists of the vertices $w_{0}, w_{1}, \ldots, w_{k}$ where it may happen that some of the vertices occur more than once. The path is simple if every vertex on the path occurs only once. Occasionally, a path as above is denoted as containing the vertices $w_{0}, w_{1}, \ldots, w_{k}$ as the sequence of edges it consists of, such that $\left(w_{0}, w_{1}\right),\left(w_{1}, w_{2}\right), \ldots,\left(w_{k-1}, w_{k}\right)$ or simply as $w_{0} \rightarrow w_{1} \rightarrow \ldots \rightarrow w_{k}$. The number of edges contained in the sequences $w_{0}, w_{1}, \ldots, w_{k}$ are called the length of the path.

Furthermore, by means of "path", an important type of connectedness in digraphs is described by the following. Two vertices, $w_{0}$ and $w_{k}$ are said to be strongly connected if there is both a path from $w_{0}$ to $w_{k}$ and a path from $w_{k}$ to $w_{0}$. A closed path is a path in which the initial and final vertices are the same. A closed path is said to be a cycle if one reaches going along the path no vertex, other than the initial-final vertex, more than once. The number of edges contained in a cycle defines the length of this cycle. Cycles of length 1 are called self cycles. A set of vertex disjoint cycles are said to be a cycle family.

\subsubsection{Controllability via Connectability}

Hereafter a graphical criterion to characterize the structural controllability is presented [Reinschke 1984,1]. 


\section{Definition 2.5.1 (Input connectable):}

Given system structural graph of a linear system with structure matrices $([A],[B])$, there should be at least one direct path from each of the input variable/s to each of the state variables

The following theorem is applied to check the controllability of a structured linear system.

\section{Theorem 2.5.1:}

A linear system with structure matrices $([A],[B],[C])$ is structurally controllable if:

1. Matrix $[A]$ is of full structural rank, and

2. The system structural graph is input connectable

\subsubsection{Observability via Connectability}

Hereafter a graphical criterion to characterize the structural controllability is presented [Reinschke 1984].

\section{Definition 2.5.2 (Output connectable):}

Given a system structural graph of a linear system with structure matrices $([A],[C])$, there should be at least one direct path from each of the state to all of the output variable/s.

The following theorem is applied to check the controllability of a structured linear system.

\section{Theorem 2.5.2:}

A linear system with structure matrices $([A],[B],[C])$ is structurally observable if:

1. Matrix $[A]$ is of full structural rank, and

2. The system structural graph is output connectable 


\subsubsection{Example}

Consider an LTI system described by Equation 2.7.

$$
\begin{aligned}
& {\left[\begin{array}{l}
\dot{x}_{1} \\
\dot{x}_{2}
\end{array}\right]=\left[\begin{array}{ll}
A_{11} & A_{21} \\
A_{12} & A_{22}
\end{array}\right]\left[\begin{array}{l}
x_{1} \\
x_{2}
\end{array}\right]+\left[\begin{array}{l}
B 1_{1} \\
B 1_{2}
\end{array}\right] u} \\
& {\left[\begin{array}{l}
y_{1} \\
y_{2}
\end{array}\right]=\left[\begin{array}{ll}
1 & 0 \\
0 & 1
\end{array}\right]\left[\begin{array}{l}
x_{1} \\
x_{2}
\end{array}\right]}
\end{aligned}
$$

The graph $G=(V, E)$ that represents the state space model of Equation 2.7 can be defined as follows. The vertex set $V$ is given by $U \cup X \cup Y$ with $U=\left\{B 1_{1}, B 1_{2}\right\}$ the set of input vertices, $X=\left\{x_{1}, x_{2}\right\}$ the set of state vertices, and $Y=\left\{y_{1}, y_{2}\right\}$ the set of output vertices. Denoting $\left(v, v^{\prime}\right)$ for a directed edge from the initial vertex $v \in V$ to the terminal vertex $v^{\prime} \in V$, the edge set $E$ is described by $E_{A} \cup E_{B} \cup E_{C}$ with $E_{A}=\left\{\left(x_{j}, x_{i}\right)\left[[A]_{i, j} \neq 0\right\}\right.$, $E_{B}=\left\{\left(u_{j}, x_{i}\right)[B]_{i, j} \neq 0\right\}$ and $E_{C}=\left\{\left(x_{j}, y_{i}\right)[C]_{i, j} \neq 0\right\}$. Figure 6 depicts the obtained digraph.

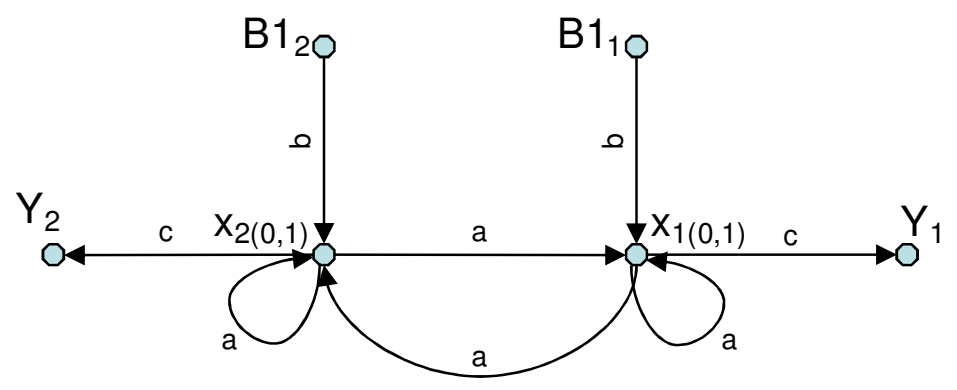

Figure 6: Digraph of Equation 2.7

Based on the obtained digraph, a structural property analysis can be performed. From observation, it can be determined that the given LTI system is indeed both controllable and observable according to Theorem 2.5.1 and 2.5.2. 


\section{Chapter Three}

\section{THERMAL DYNAMIC SYSTEM MODEL}

A dynamic model of a system as well as its properties forms the background of any control design activity [Bokor, Hangos, and Szederkényi 2004, 1]. This chapter focuses on the theoretical basis for thermal dynamic system model's development, which is utilized for control design purposes. The theories that are covered include the Thermal Network approach which is utilized to derive mathematical equations governed by the first law of thermodynamics principle; The Nonlinear State Space model which is utilized for control design analysis; The Structured Model which is utilized for control design analysis from a system structure point of view, and the Digraph which is utilized to model the system graphically. Each of of these modeling techniques forms a sequence that is required in order to obtain a model that is utilized for system structural properties analysis using graph theory. 


\subsection{Thermal Dynamic Model for Control Purposes}

A modeling task is specified by giving the description of the system to be modeled together with the modeling goal, that is, the intended use of the model [Cameron and Hangos 2001, 19-40]. The modeling goal for the most part determines the model, its variable, spatial and time characteristics, as well as its resolution or level of detail and precision. In the case of this research, where the goal of the modeling is to obtain a mathematical model that can be useful for control system design of a thermal dynamic system, it is suggested that the main requirement of the model is the ability to capture the time characteristics, such a time constants.

Given the goal of the modeling effort, thermal network modeling approach is selected. This approach is very intuitive and allows a systematic formulation and solution of general and complicated problems. Furthermore, in order to obtain a finite dimensional system model that is adequate in handling control design, lumping the thermal dynamic parameters is also suggested. In thermal dynamic system, the lumped parameter is termed as balance volume or lump which has properties containing only one-phase that is assumed to be perfectly mixed and isothermal [Bokor, Hangos, and Szederkényi 2004, 1]. This lumped parameter model results in a mathematical model that is composed of systems of ordinary differential equations (ODEs) and is often coupled with many nonlinear and linear algebraic constraints. The total system is referred to as a differential-algebraic equation set (DAE) and it is governed by the first law of thermodynamics, that is, conservation balances for energy. In this case, the thermal 
dynamic DAE is developed by following the conservation of energy principles. The general conservation balance for total energy over the balance volume is given by:

$$
\left\{\begin{array}{l}
\text { Rate of change } \\
\text { of total energy }
\end{array}\right\}=\left\{\begin{array}{l}
\text { flow of energy } \\
\text { into the system }
\end{array}\right\}-\left\{\begin{array}{l}
\text { flow of energy } \\
\text { out the system }
\end{array}\right\}+\left\{\begin{array}{l}
\text { Sink } \\
\text { system }
\end{array}\right\}
$$

Furthermore, the linear algebraic part of the model is called constitutive equation, which is obtained by identifying different means of energy transport mechanisms that should be included in the model. This mechanism is referred to the three fundamentals of heat transfer:

1. Conduction is an energy transport mechanism as a result of molecular-level kinetic energy transfer in solids, liquids and gases. The difference between the thermal dynamical state variables, that is, temperature in the two phases is the driving force for the transport.

2. Convection is an energy transport mechanism that is carried by the transport of larger-scale motions of a fluid, either liquid or gas. The convection of a lumped thermal dynamic system is represented by the inflows and outflows of the lumped parameter or balance volumes.

3. Radiation is an energy transport mechanism that is established by electromagnetic waves. For radiation to occur, there needs to be two surfaces with two different temperatures.

Each of these mechanisms can be represented by specific forms of constitutive equations with basic property that are assumed to be strictly-additive, which is very essential in constructing the energy conservation balance equation of a thermal dynamic.

The next few sections describe the model development process of a nonlinear thermal dynamic system. This particular modeling approach is the starting point in 
developing a new structural property analysis method of controllability and observability for large-scale structured nonlinear thermal dynamic systems:

1. Thermal Networks. A brief overview of a concept for constructing a thermal dynamic model for control purposes is discussed here.

2. State space models of Thermal Dynamic Systems. Basic notions of state space representation for a nonlinear system are reviewed. This includes: a decomposition of the state equations driven by the mechanism taking place in the thermal dynamic system.

3. Structured System of Thermal Dynamic Systems. The thermal dynamic system is a good example of a model that captures most of the structurally available information from physical laws. Therefore, the concept of a structured system for a thermal dynamic system is presented here.

4. Digraph of Nonlinear Thermal Dynamic System. Given that the goal of this research is to develop a graphical theoretic approach for controllability and observability analysis, it is fundamental to understand how a mathematical model is converted to a graphical model. This approach will be presented in this section.

\subsection{Thermal Networks}

Thermal network approach approximates a thermal dynamic system as being composed of a finite number of parts $N$, called nodes, each of which represents a balance volume or lump [Bokor, Hangos, and Szederkényi 2004, 1]. 


\section{Definition 3.2.1 (Balance Volume):}

Parts of a lumped thermal dynamic system which contain only one phase and can be assumed to be perfectly mixed will be termed balance volumes or lumps. It is also assumed that each node is to be isothermal.

To model heat exchange, the nodes are connected by resistance, thus forming a thermal network. Neighboring nodes are nodes that are directly coupled by conduction, convection, or radiation. The heat flow between neighbors is given by Equation 3.2:

$$
\dot{Q}_{n^{\prime}-n}=\frac{T_{n^{\prime}}-T_{n}}{R_{n^{\prime} n}}
$$

where $R_{n^{\prime} n}$ is the resistance between $n^{\prime}$ and $n$ and it is used to represent three different heat transfer mechanisms: conduction, convection and radiation. In addition, there may be direct heat input $\dot{Q}_{n}$ at node $n$, from heat sources such as solar radiation, an electric heater, or boiler. Furthemore, the heat capacity of node $n$ is denoted by $C_{n}$ while its temperature is indicated by $T_{n}$.

Assuming constant $C_{n}$, the rate of change of the heat stored in node $n$ is $C_{n} \dot{T}_{n}$, and by the first law of thermodynamics it must be equal to the total rate of heat input. Thus the heat balance of node $n$ is a first-order differential equation in $T_{n}$ :

$C_{n} \dot{T}_{n}=\sum_{n^{\prime}=1}^{N} \frac{T_{n^{\prime}}-T_{n}}{R_{n^{\prime} n}}+\dot{Q}_{n}$

As for signs, it is noted that $T_{n^{\prime}}-T_{n}$ is positive; heat flows from $n^{\prime}$ to $n$ making a positive contribution to $\dot{T}_{n}$. In most cases, a given node can interact directly with only a relatively small number of nodes, and so the number of nonzero terms in this sum is much smaller than $N$. 
Equation 3.3, one for each node, forms a system of $N$ first-order differential equations with $N$ unknowns, that is, the node temperature $T_{n}$. By analogy with electric circuits it is convenient to represent a thermal network by diagrams:

1. Electronic capacitor represents a capacitance $C$

2. Electronic resistor represents a resistance $R$

3. Temperature $T$ are analogous to voltages

4. Heat flow $\dot{Q}$ are analogous to currents

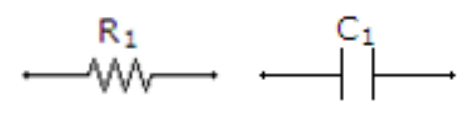

\section{Figure 7: Resistance $\mathbf{R}$ and Capacitance $\mathbf{C}$}

There is one to one correspondence between the diagram and the set of equations of thermal network. The diagram has the advantage of being much easier to grasp, but the equation is needed for finding the solutions. Once the diagram has been drawn, one can easily write down the equations where there is one first-order differential equation for each node.

To illustrate the application of thermal network approach, consider a heat exchanger as depicted by Figure 8 . 


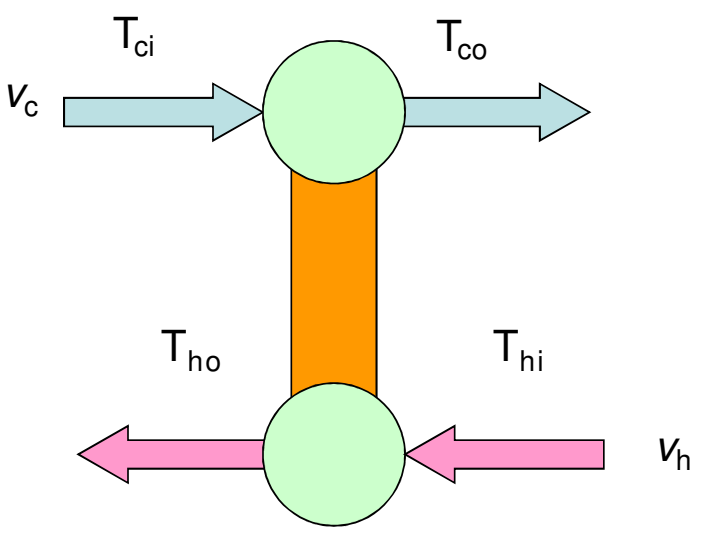

Figure 8: Heat Exchanger

The heat exchanger is one of the widely used thermal dynamic systems, which is utilized for energy exchange between at least two fluid phase streams, hot and cold streams. While the heat exchanger is usually considered a distributed thermal dynamic system, it is acceptable to build and approximate a lumped parameter model using finite difference approximations of their spatial variables. A heat exchanger can then be seen as a composite lumped parameter thermal dynamic system consisting of elementary dynamic units as depicted in Figure 8. Here, a heat exchanger consists of two perfectly stirred balance volumes (lumps) connected by a heat conducting wall. One lump is called the hot $(h)$ and the other one the cold $(c)$. Using the thermal network approach, the following electric circuit that represents the heat transfer mechanism of a heat exchanger is obtained and is illustrated by Figure 9 . 


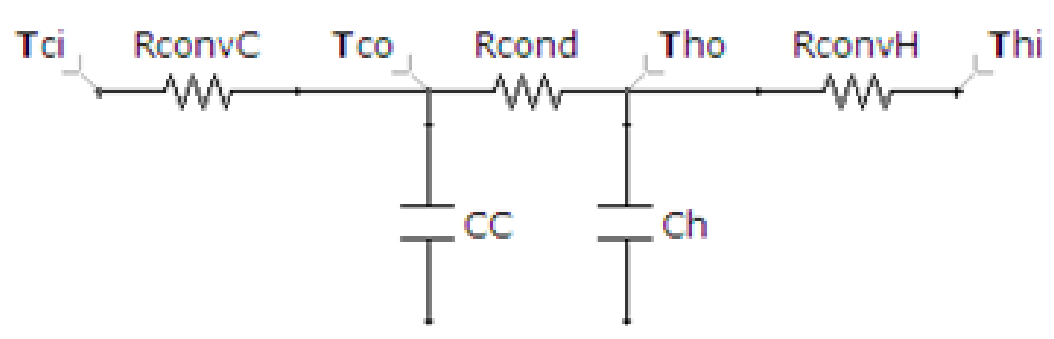

Figure 9: Circuit Model of a Heat Exchanger

Subsequently, the following DAE set is derived:

$$
\begin{gathered}
C_{c} \dot{T}_{c o}=\frac{T_{c o}-T_{h o}}{R_{c o n d}}+\frac{T_{c i}-T_{c o}}{R_{c o n v C}} \\
C_{h} \dot{T}_{h o}=\frac{T_{h o}-T_{c o}}{R_{c o n d}}+\frac{T_{h i}-T_{h o}}{R_{\text {conv }}}
\end{gathered}
$$

Furthermore, to obtain a complete and detailed DAE, the algebraic constitutive equation is incorporated. The following is the constitutive equation for the heat exchanger example:

$C_{j}=c p_{j} \rho_{j} V_{j}$ where balance volume/lump $j=h, c$

$R_{\text {cond }}=\frac{1}{U A}$

$R_{c o n v j}=\frac{1}{\dot{m}_{j} c p_{j}}$

where:

$c p_{j}:$ Specific heat of lump $j$

$\rho_{j}$ : Density of lump $j$

$V_{j}:$ Volume of lump $j$

$U:$ Constant heat transfer coefficient (conduction) 


\section{$A$ : Contact surface area}

$\dot{m}_{j}$ : mass flow rate at lump $j$

$T_{j i}$ : inlet temperature of lump $j$

$T_{j o}:$ outlet temperature of lump $j$

Substituting these constitutive equations into Equation 3.4 and 3.5, the following complete mathematical model of a heat exchanger is obtained:

$$
\begin{aligned}
& \left(c_{p_{c}} \rho_{c} V_{c}\right) \dot{T}_{c o}=(U A)\left(T_{c o}-T_{h o}\right)+\left(\dot{m}_{c} c_{p_{c}}\right)\left(T_{c i}-T_{c o}\right) \\
& \left(c_{p_{h}} \rho_{h} V_{h}\right) \dot{\Gamma}_{h o}=(U A)\left(T_{h o}-T_{c o}\right)+\left(\dot{m}_{h} c_{p_{h}}\right)\left(T_{h i}-T_{h o}\right)
\end{aligned}
$$

\subsection{State Space of Thermal Dynamic}

The law of conservation of energy has made the state space models a natural representation of thermal dynamic model equation as it allows an easy transformation between the two. Not only that, with state space form, the clear engineering meaning of the original thermal dynamic model equation is well maintained.

State, which is the base of the state-space model, is defined as the smallest possible subset of a system that can represent the entire state of the system at any given time. Thus, instead of describing a system as an operator mapping from the input space to the output space using the entire input-output history and the planned input to calculate future outputs, new information called state of the system at time $t_{0}$ is used. The state of the system at time $t_{0}$ includes all past information up to time $t_{0}$, initial condition for the 
outputs, as well as its derivatives and its past input history. Therefore, in order to calculate all future values output, that is $y(t)$ for $t \geq t_{0}$, only input $u(t)$ for $t \geq t_{0}$ and the state $x(t)$ at $t=t_{0}$ is needed. In summary, the state-space model is defined as a model that uses the concept of state.

A general state-space model is composed by two sets of equations:

1. State equations are a set of time-dependent ordinary differential equations that describe the evolution of the states as a function of the input and state variables. In order to describe a system, it requires a finite number of state equations and the same number of state variables. Thus, it is called finite dimensional system.

2. Output equations are a set of algebraic equations that describe the relation between the value of the output signals to the state and the input signals.

The general form of state-space models of continuous linear time invariant (LTI) system is described by Equation 3.8:

$\Sigma= \begin{cases}\dot{x}(t)=f(x, u)=A x(t)+B u(t) & ;(\text { State }- \text { Equation }) \\ y(t)=g(x)=C x(t) & ;(\text { Output }- \text { Equation })\end{cases}$

Given the initial condition $x\left(t_{o}\right)=x(0), \quad x(t) \in \mathfrak{R}^{n}, \quad y(t) \in \mathfrak{R}^{p}, u(t) \in \mathfrak{R}^{l}$ represent the state, output, and input vectors of finite dimensional space; and $A \in \mathfrak{R}^{n \times n}$, $B \in \mathfrak{R}^{n \times l}, C \in \mathfrak{R}^{p \times n}$ represent time-constant, input and output matrices with constant (time-independent) elements.

\section{Definition 3.3.1 (Linear State Space Representation):}

The state space representation (SSR) that is described by Equation 3.8 is the triplet of constant matrices $(A, B, C)$. The dimension of an SSR is the dimension of the state vector: $\operatorname{dim}[x(t)]=n$. The state space $\chi$ is the set of all states: 
$x(t) \in \chi, \operatorname{dim}[\chi]=n$.

Continuing the previous example of a heat exchanger, Equation 3.9 and 3.10 are the continuous time state equations of the heat exchanger which follow the energy conservation balances:

$$
\begin{aligned}
& \dot{T}_{h o}=\frac{U A}{c_{p_{h}} \rho_{h} V_{h}}\left(T_{h o}-T_{c o}\right)+\frac{v_{h}}{V_{h}}\left(T_{h i}-T_{h o}\right) \\
& \dot{T}_{c o}=\frac{U A}{c_{p_{c}} \rho_{c} V_{c}}\left(T_{c o}-T_{h o}\right)+\frac{v_{c}}{V_{c}}\left(T_{c i}-T_{c o}\right)
\end{aligned}
$$

Subsequently, assuming that the heat exchanger is fully observable, that is, system output is measurable, Equation 3.9 and 3.10 become the following state space equation:

$$
\begin{aligned}
& \dot{x}=\left[\begin{array}{cc}
-\frac{v_{c}}{V_{c}}-\frac{U A}{c_{p_{c}} \rho_{c} V_{c}} & \frac{U A}{c_{p_{c}} \rho_{c} V_{c}} \\
\frac{U A}{c_{p_{h}} \rho_{h} V_{h}} & -\frac{v_{h}}{V_{h}}-\frac{U A}{c_{p_{h}} \rho_{h} V_{h}}
\end{array}\right] x+\left[\begin{array}{cc}
\frac{v_{c}}{V_{c}} & 0 \\
0 & \frac{v_{h}}{V_{h}}
\end{array}\right] u \\
& y=\left[\begin{array}{ll}
1 & 0 \\
0 & 1
\end{array}\right] x
\end{aligned}
$$

where $x=\left[\begin{array}{l}x_{1} \\ x_{2}\end{array}\right]=\left[\begin{array}{l}T_{c o} \\ T_{h o}\end{array}\right], u=\left[\begin{array}{l}u_{1} \\ u_{2}\end{array}\right]=\left[\begin{array}{l}T_{c i} \\ T_{h i}\end{array}\right]$, and $y=\left[\begin{array}{l}y_{1} \\ y_{2}\end{array}\right]=\left[\begin{array}{l}x_{1} \\ x_{2}\end{array}\right]=\left[\begin{array}{l}T_{c o} \\ T_{h o}\end{array}\right]$.

In a realistic case, however, a linear model of the heat exchanger is not valid as the flow rates are the controllable input variables. Hence, a nonlinear system is considered and its general form of continuous time state space models is described rather differently. Nonlinear finite dimensional system represents a wide class of nonlinear systems. However, in the case of this research, lumped thermal dynamic models derived from first engineering principles are considered here. The general form of state space models of finite dimension nonlinear systems is described by Equation 3.12: 
$\Sigma= \begin{cases}\dot{x}(t)=\tilde{f}(x(t), u(t)) & ;(\text { State }- \text { Equation }) \\ y(t)=\tilde{h}(x(t), u(t)) & ;(\text { Output }- \text { Equation })\end{cases}$

with the state, input and output vectors $x, u, y$ and with the smooth nonlinear mappings $\tilde{f}: \Re^{n} \times \mathfrak{R}^{m} \mapsto \mathfrak{R}^{n}, \tilde{h}: \mathfrak{R}^{n} \times \mathfrak{R}^{m} \mapsto \mathfrak{R}^{p}$. If the nonlinear functions $\tilde{f}$ and $\tilde{h}$ above are in a special form, an input-affine form is obtained:

$\Sigma= \begin{cases}\dot{x}(t)=\tilde{f}(x(t), u(t))=g_{0}(x(t))+\sum_{i=1}^{m} g_{i}(x(t)) u_{i}(t) & ;(\text { State }- \text { Equation }) \\ y(t)=\tilde{h}(x(t))=h(x(t)) & ;(\text { Output }- \text { Equation })\end{cases}$

with the same state, input, and output vector $x, u$, and $y$ as above, and with the smooth nonlinear mappings $g_{i}: \Re^{n} \mapsto \mathfrak{R}^{n}$ for $i=0,1, \ldots, m, h: \Re^{n} \mapsto \mathfrak{R}^{p}$. Hence, the nonlinear state space model of the heat exchanger becomes the following: $u=\left[\begin{array}{l}u_{1} \\ u_{2}\end{array}\right]=\left[\begin{array}{l}v_{c} \\ v_{h}\end{array}\right]$,

with simplification of $k_{h}=\frac{U A}{c_{p_{h}} \rho_{h} V_{h}}$ and $k_{c}=\frac{U A}{c_{p_{c}} \rho_{c} V_{c}}$

The nonlinear state equation 3.9 and 3.10 becomes:

$\dot{T}_{c o}=-k_{c} x_{1}(t)+k_{c} x_{2}(t)+\left(\frac{T_{c i}}{V_{c}}-\frac{x_{1}}{V_{c}}\right)\left(v_{c}(t)\right)$

$\dot{T}_{h o}=-k_{c} x_{1}(t)+k_{c} x_{2}(t)+\left(\frac{T_{h i}}{V_{h}}-\frac{x_{2}}{V_{h}}\right)\left(v_{h}(t)\right)$

Subsequently, the nonlinear state space matrices of Equation 3.13 would have the following:

$g_{o}(x)=\left[\begin{array}{cc}-k_{c} & k_{c} \\ k_{h} & -k_{h}\end{array}\right]\left[\begin{array}{c}x_{1} \\ x_{2}\end{array}\right], \quad g_{1}(x)=\left[\begin{array}{c}\frac{T_{c i}}{V_{c}}-\frac{1}{V_{c}} x_{1} \\ 0\end{array}\right]$ and $g_{2}(x)=\left[\begin{array}{c}0 \\ \frac{T_{h i}}{V_{h}}-\frac{1}{V_{h}} x_{2}\end{array}\right]$, 
With the notation above the state equation 3.13 can be written in the following general form:

$$
\dot{x}=A^{0} x+\left(\sum_{i=1}^{2} A^{i} x+B^{i} b\right) u_{i}
$$

In the above equation one can clearly see the origin of the terms on the right-hand side:

1. Linear state transfer term or drift term/conduction:

$$
A^{0}=\left[\begin{array}{cc}
-\frac{U A}{c_{p_{c}} \rho_{c} V_{c}} & \frac{U A}{c_{p_{c}} \rho_{c} V_{c}} \\
\frac{U A}{c_{p_{h}} \rho_{h} V_{h}} & -\frac{U A}{c_{p_{h}} \rho_{h} V_{h}}
\end{array}\right]=\left[\begin{array}{cc}
-k_{c} & k_{c} \\
k_{h} & -k_{h}
\end{array}\right]
$$

2. Bilinear state convection term originating from the output convection with

$$
A^{1}=\left[\begin{array}{cc}
-\frac{1}{V_{c}} & 0 \\
0 & 0
\end{array}\right], A^{2}=\left[\begin{array}{cc}
0 & 0 \\
0 & -\frac{1}{V_{h}}
\end{array}\right]
$$

3. Linear input term originating from the input convection with

$$
B^{1}=\left[\begin{array}{cc}
\frac{1}{V_{c}} & 0 \\
0 & 0
\end{array}\right] B^{2}=\left[\begin{array}{cc}
0 & 0 \\
0 & \frac{1}{V_{h}}
\end{array}\right] \text { where } b=\left[\begin{array}{l}
b_{1} \\
b_{2}
\end{array}\right]=\left[\begin{array}{l}
T_{c i} \\
T_{h i}
\end{array}\right]
$$

\subsection{Structured System}

In the framework of the traditional control theory of nonlinear systems, the entries of the state-space matrices $\left(A^{i}, B, C\right)$ of an input-affine nonlinear system are regarded as numerical data given with 100 percent precision. For physical reasons, however, the parameters involved in the entries of $A^{i}, B$, and $C$ are only approximately known. 
Consequently, it is adequate to regard the entries of $A^{i}, B$ and $C$ as indeterminate. Only some entries which are often precisely zero have exact numerical values.

In the context of "controllability and observability of a structured system," utilizing only the "structure" of the matrices $A^{i}, B$ and $C$ is proposed in this research. This means, instead of numerically given matrices $A^{i}, B$ and $C$, the corresponding structure matrices $\left[A^{i}\right],[B]$, and $[C]$ of the same dimensions are considered. The following definition is suggested for this research.

\section{Definition 3.4.1 (Structured Matrix):}

The elements of a structure matrix $\left[A^{k}, B^{k}, C\right]$ are either fixed at zero or indeterminate values which are assumed to be independent of one another.

$$
\begin{gathered}
\left\{A^{k}\right\}_{i, j}=\left\{\begin{array}{cl}
0 & \text { if } A_{i j}^{k}=0, \\
e_{i, j}^{k} & \text { otherwise }
\end{array}\right. \\
\left\{B^{k^{\prime}}\right\}_{i, j}=\left\{\begin{array}{cl}
0 & \text { if } B_{i j}^{k}=0, \\
e_{i, j}^{b^{m}} & \text { otherwise }
\end{array}\right. \\
\{C\}_{i, j}=\left\{\begin{array}{cl}
0 & \text { if } C_{i j}=0, \\
e_{i, j}^{c} & \text { otherwise }
\end{array}\right.
\end{gathered}
$$

\section{Definition 3.4.2 (Signed Structured Nonlinear Thermal Dynamic System with Drift):}

The elements of a structure matrix $\left\{A^{k}, B^{k}, C\right\}$ are either fixed at zero or indeterminate values which are assumed to be independent of one another.

$$
\left\{A^{k}\right\}_{i, j}=\left\{\begin{array}{lll}
0 & \text { if } & A_{i j}^{k}=0, \\
+e_{i, j}^{k} & \text { if } & A_{i j}^{k}>0, \\
-e_{i, j}^{k} & \text { if } & A_{i j}^{k}<0,
\end{array}\right.
$$




$$
\begin{gathered}
\left\{B^{k^{\prime}}\right\}_{i, j}=\left\{\begin{array}{lll}
0 & \text { if } & B_{i j}^{k}=0, \\
+e_{i, j}^{b^{m}} & \text { if } & B_{i j}^{k}>0, \\
-e_{i, j}^{b^{m}} & \text { if } & B_{i j}^{k}<0,
\end{array}\right. \\
\{C\}_{i, j}=\left\{\begin{array}{lll}
0 & \text { if } & C_{i j}=0, \\
+e_{i, j}^{c} & \text { if } & C_{i j}>0, \\
-e_{i, j}^{c} & \text { if } & C_{i j}<0,
\end{array}\right.
\end{gathered}
$$

Following the previous example of a heat exchanger, the following matrices of a structured system model of Equation 3.16 is obtained:

1. Linear state transfer term or drift term/conduction:

$$
A^{0}=\left[\begin{array}{ll}
e_{1,1}^{0} & e_{1,2}^{0} \\
e_{2,1}^{0} & e_{2,2}^{0}
\end{array}\right]
$$

2. Bilinear state convection term originating from the output convection with

$$
A^{1}=\left[\begin{array}{cc}
e_{1,1}^{1} & 0 \\
0 & 0
\end{array}\right], A^{2}=\left[\begin{array}{cc}
0 & 0 \\
0 & e_{2,2}^{2}
\end{array}\right]
$$

3. Linear input term originating from the input convection with

$$
B^{1}=\left[\begin{array}{cc}
e_{1,1}^{b^{1}} & 0 \\
0 & e_{2,2}^{b^{2}}
\end{array}\right], B=\left[\begin{array}{cc}
e_{1,1}^{b^{1}} & 0 \\
0 & e_{2,2}^{b^{2}}
\end{array}\right] \text { and } C=\left[\begin{array}{cc}
e_{1,1}^{c} & 0 \\
0 & e_{2,2}^{c}
\end{array}\right]
$$

If a structured Nonlinear Thermal Dynamic System with Drift model is considered, the matrices of Equation 3.16 become the following:

1. Linear state transfer term or drift term/conduction:

$$
A^{0}=\left[\begin{array}{cc}
-e_{1,1}^{0} & e_{1,2}^{0} \\
e_{2,1}^{0} & -e_{2.2}^{0}
\end{array}\right]
$$


2. Bilinear state convection term originating from the output convection with

$$
A^{1}=\left[\begin{array}{cc}
-e_{1,1}^{1} & 0 \\
0 & 0
\end{array}\right], A^{2}=\left[\begin{array}{cc}
0 & 0 \\
0 & -e_{2,2}^{2}
\end{array}\right]
$$

3. Linear input term originating from the input convection with

$$
B^{1}=\left[\begin{array}{cc}
e_{1,1}^{b^{1}} & 0 \\
0 & e_{2,2}^{b^{2}}
\end{array}\right], B=\left[\begin{array}{cc}
e_{1,1}^{b^{1}} & 0 \\
0 & e_{2,2}^{b^{2}}
\end{array}\right] \text { and } C=\left[\begin{array}{cc}
e_{1,1}^{c} & 0 \\
0 & e_{2,2}^{c}
\end{array}\right]
$$

Note that in the case of a structured thermal dynamic system models which follow the form of Equation 3.16, the following equalities are valid:

1. At node $i, e_{i, i}^{0}=-e_{i, j}^{0}$, for $j=1, \ldots, n$, except $i=j$

2. At node $i, e_{i, j}^{k}=-e_{i, j}^{b^{k}}$ for $j=1, \ldots, n$, except $i=j$ and $k=1, \ldots, m$

where $n$ is the dimension of state space model and $m$ is the number of input variables.

\subsection{Digraph of Nonlinear System}

Many results on structured systems are related to the graph theoretic approach. However, this approach is mainly dedicated to linear systems. Structural properties of linear system such as controllability and observability, as well as solvability of classical control problems such as disturbance rejection, input-output decoupling, fault detection, and isolation, are studied using the graph theoretic approach. Survey paper [Commault, Dion, and Van der Woude 2003, 1125-1144] reviews the most significant results in this area. From these studies, it follows that graph-theoretic approach provides simple and elegant solutions and so is very well suited to analyze large-scale or/and uncertain 
systems. Unfortunately, not so many works based on graph-theoretic methods deal with nonlinear systems. This section is devoted to the definition of a digraph which represents a structured bilinear system.

The graph $G=(V, E)$ of a structured bilinear system represented by its state space model of equation 3.16 is defined by a vertex set $V$ and an edge set $E$. The vertex set $V$ is given by $B \cup X \cup Y$ with $B=\left\{b_{1}^{1}, \ldots, b_{n}^{m}\right\}$ the set of fixed- input vertices associated with state vertex $i$ and input variable $m, X=\left\{x_{1}, \ldots, x_{n}\right\}$ the set of state vertices, $Y=\left\{y_{1}, \ldots, y_{p}\right\}$ the set of output vertices. Denoting $\left(v, v^{\prime}\right)$ for a directed edge from the initial vertex $v \in V$ to the terminal vertex $v^{\prime} \in V$, the edge set $E$ is described by $E_{A^{l}} \cup E_{B^{l}} \cup E_{C}$ with $E_{A^{l}}=\left\{\left(x_{j}, x_{i}\right)\left[\left[A^{l}\right]_{i, j} \neq 0\right\} \quad l=0,1, \ldots, m, E_{B^{l}}=\left\{\left(u_{j}, x_{i}\right)\left[B^{l^{l}}\right]_{i, j} \neq 0\right\}\right.$ for $l^{\prime}=1, \ldots, m$ and $E_{C}=\left\{\left(x_{j}, y_{i}\right)[C]_{i, j} \neq 0\right\}$. In the latter, for instance $\left[A^{l}\right]_{i, j} \neq 0$ means that the $(i, j) t h$ entry of the matrix $\left\lfloor A^{l}\right\rfloor$ is a non-zero parameter. Moreover, for $l=0,1, \ldots, m$ and $l^{\prime}=1, \ldots, m$, an index $l$ is assigned to each edge $e \in E_{A^{\prime}} \cup E_{B^{\prime}}$. Note that several indexes may be given to an edge $e$ if it belongs to several subsets $\left(E_{A^{l}} \cup E_{B^{\prime}}\right)$-edges. For $l=l^{\prime}=1, \ldots, m$, this index correspond to system input $u_{l}$. This completes how a structured bilinear system is being represented by a digraph.

Furthermore, denote $W, W^{\prime}$ being two nonempty subsets of the vertex set $V$ of the graph $G$. A path exists from $W$ to $W^{\prime}$ if there is an integer $k$ and there are vertices $w_{0}, w_{1}, \ldots, w_{k} \in V$ such that $w_{0} \in W, w_{k} \in W^{\prime}$ and $\left(w_{i-1}, w_{i}\right) \in E$ for $i=1,2, \ldots, k$. The vertex $w_{0}$ is called the beginning vertex. The vertex $w_{k}$ is called the end vertex. It is said that the path consists of the vertices $w_{0}, w_{1}, \ldots, w_{k}$, where it may happen that some of 
the vertices occur more than once. It also said that each of the vertices in $w_{0}, w_{1}, \ldots, w_{k}$ is contained in the path. The path is called simple if every vertex on the path occurs only once. Occasionally, a path described above is containing the vertices $w_{0}, w_{1}, \ldots, w_{k}$ as the sequence of edges it consists of, that is $\left(w_{0}, w_{1}\right),\left(w_{1}, w_{2}\right), \ldots,\left(w_{k-1}, w_{k}\right)$ or simply as $w_{0} \rightarrow w_{1} \rightarrow \ldots \rightarrow w_{k}$. The number of edges contained in the sequences $w_{0}, w_{1}, \ldots, w_{k}$ is called the length of the path.

By means of path, several important types of connectedness in digraphs are defined. Two vertices, $w_{0}$ and $w_{k}$ are said to be strongly connected if there is both a path from $w_{0}$ to $w_{k}$ and a path from $w_{k}$ to $w_{0}$. A closed path is a path whose initial and final vertices are the same. A closed path is said to be a cycle if one reaches going along the path no vertex, other than the initial-final vertex, more than once. The number of edges contained in a cycle defines the length of this cycle. Cycles of length 1 are called self cycles. A set of vertex disjoint cycles is said to be a cycle family.

Using the previous example of the heat exchanger, a digraph now can be derived. Figure 10 depicts the digraph of the previous example of the heat exchanger.

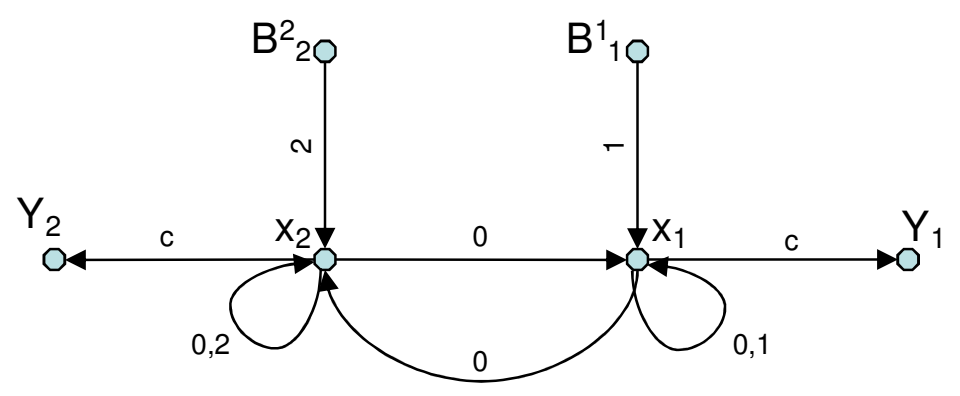

Figure 10: Digraph of Heat Exchanger 


\section{Chapter Four}

\section{BUILDING COMBINED HEAT AND POWER SYSTEM: \\ A LARGE-SCALE NON-LINEAR THERMAL DYNAMIC SYSTEM}

This chapter focuses on a mathematical model development of a real case system which fits the definition of a large-scale system. Rather than directly modeling the system as a whole, the system is modeled by first decoupling it along the physical boundaries of its subsystems which subsequently combining to form a large-scale system. Chapter four is used as the basis theory to construct a mathematical model of each subsystem as well as a state space and a digraph model of the large-scale system after all subsystems are integrated. The process begins in section 4.1 where the description of the actual largescale system is reviewed. The mathematical model of each subsystem is derived in section 4.2. The obtained models are then combined forming a large-scale state space model in section 4.3. For structural analysis purposes, the structured system approach is imposed to the model and its digraph is constructed in section 4.4. 


\subsection{Building Combined Heat and Power: a System Description}

According to the U.S. Lodging Census Database, currently there are 48,000 lodging establishments representing about 4.4 million rooms across the fifty states and the District of Columbia as indicated on the Environmental Protection Agency's Website. With high compatibility between the energy profile of lodging facility and the type of energy that a CHP produces, it is believed that CHP package would have the potential for a profound impact on a national energy savings initiative which significantly furthers the penetration of CHP technology into the national energy spectrum. Therefore, a research project was started several years ago to investigate the optimization of CHP in conjunction with several different types of thermally activated building equipment in a specific hotel building. The goal of the research is to develop an intelligent control system that can economically control the operation of CHP and its integrated building equipment while maintaining the comfort level of building's occupants.

The building combined heat and power (BCHP) system of a hotel building, where the research project takes place, consisted of three units of CHP microturbine, hydronic radiant floor heating, and domestic water heaters which are interconnected through a glycol loop distribution system. The basic energy system, that is, CHP microturbines, heat exchangers, and control system, is housed in a $15 \times 25$ foot building located at the edge of the back parking lot of the hotel (hereafter referred to as the Test Facility). The test facility that is connected to the hotel by several electric cable runs and conduits is used as a control room/research office. Furthermore, it is also the central hub for the research activities, including data collection, and coordination of the use of the CHP energy in the hotel. 


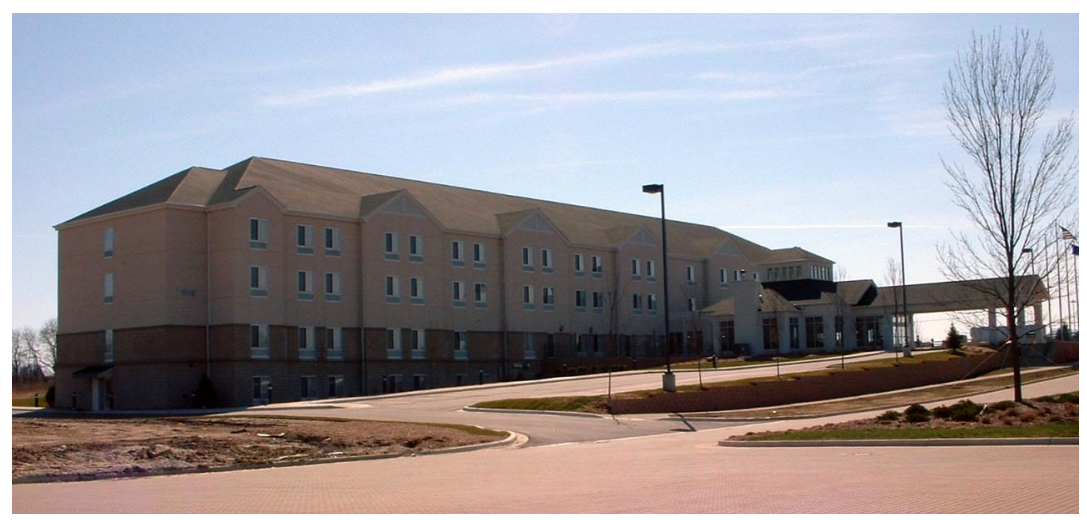

Figure 11: Hotel Building

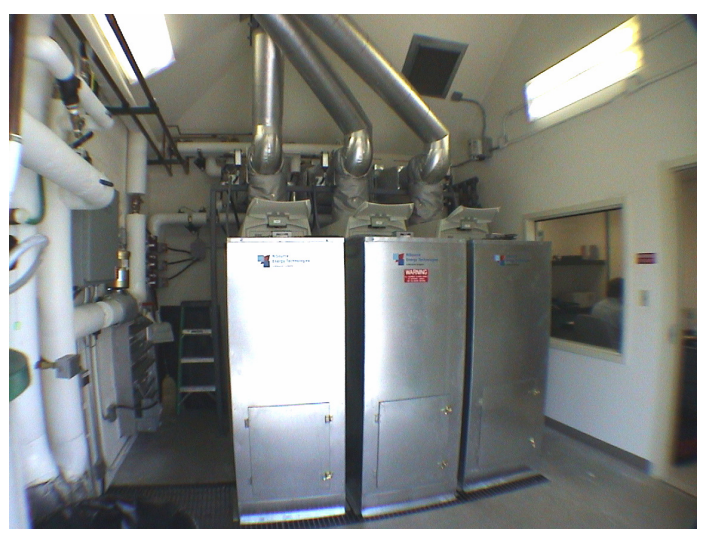

Figure 12: Inside of the Test Facility

Each unit of CHP Microturbine generates $28 \mathrm{~kW}$ at $480 \mathrm{~V}$ three-phase forming a distributed generation (DG) component of this building research. The microturbine outputs have individual 50A breaker protection and are bused together through a 200A breaker feeding a 480-208V 225kVA transformer and a 480-480V 45kVA transformer. Each transformer has a disconnect device accessible from the outside of the hotel. The $208 \mathrm{~V}$ output of the $225 \mathrm{kVA}$ transformer is connected through a transfer switch to a $200 \mathrm{~A}$ switchgear breaker and to a "protected load" panel. The output of the $480 \mathrm{~V}$ isolation transformer is connected to the $480 / 277 \mathrm{~V}$ service panel. With this configuration, the 
$480 \mathrm{~V}$ panel is supplied by the microturbines through the $480 \mathrm{~V}$ isolation transformer or through the $225 \mathrm{kVA}$ transformer if the microturbines are off. A protected load panel is supplied through the transfer switch. Figure 13 illustrates a schematic for the electric component of this CHP system.

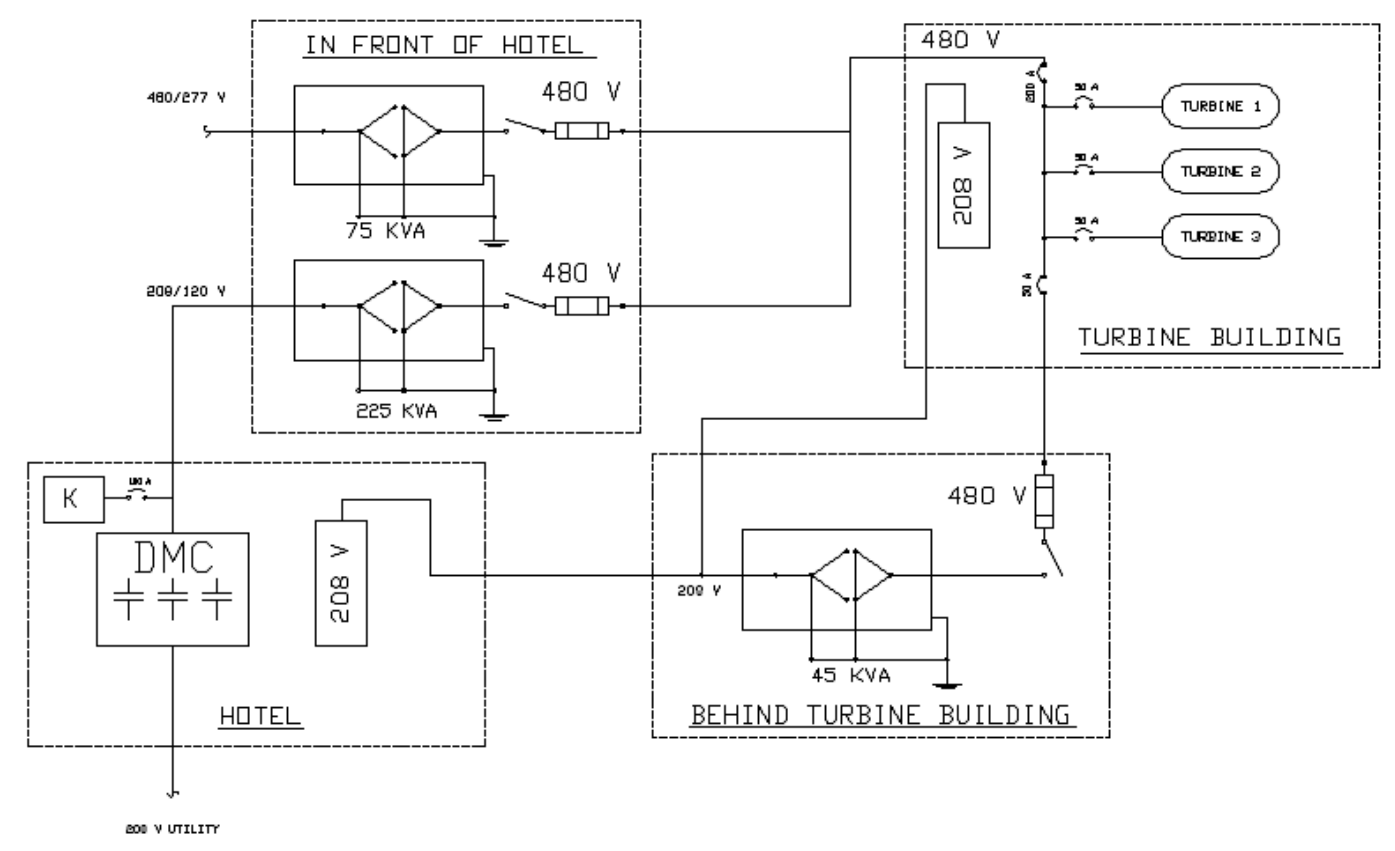

Figure 13: Electric System Design

Under full load capacity, each unit of CHP Microturbine potentially produces 200,000 Btu of thermal energy which is used to feed the building thermal activated components, such as a hydronic radiant floor heating system as well as for the whole hotel domestic water heating. The heat-exchanger of the microturbine captures the hot air that is being exhausted when microturbines generate electricity, and transfers its heat to a heat distribution system called main loop, which is served by glycol. Depending on the amount of electricity that is being generated, the temperature of the exhaust air ranges from $350^{0} \mathrm{~F}$ to $500^{0} \mathrm{~F}$. When this excess heat is transferred to the main loop, the glycol is 
heated to a temperature of $175^{\circ} \mathrm{F}$ to $185^{\circ} \mathrm{F}$, which is sufficient to activate any thermal activated equipment in a building. Figure 14 depicts the schematic diagrams of the overall BCHP system. Measurement devices are placed in a subsystem to monitor the flow rate and temperature of the hot glycol during its circulation. Note that the pool mechanical room area was not included in this research.

The hydronic radiant floor heating system is utilized to provide space heating for some portion of the area in the building which includes lobby, dining area, kitchen, and offices. The space heating system utilizes three inch diameter plastic tubing embedded in concrete floors of the building interior to distribute the heat from high temperature glycol. Conventionally, an external water heater or boiler arrangement is used. This research investigates how such subsystems can be extended to use CHP waste heat streams and thereby increase the overall building energy efficiency. The hydronic systems had to be designed and integrated together in a manner that would not interfere with the normal hotel construction procedures and schedule. Thus, prior to concrete being poured, a hydronic heating pipe in was placed in the floors. Figure 15 depicts the piping system installation of a hydronic radiant floor heating system.

There are seventeen active loops of hydronic radiant floor heating that serve the lobby, dining area, kitchen and office area of the hotel, and three inactive loops in the floor of the indoor swing pool area. Several measurement units were embedded evenly across the floor of these areas to monitor the temperature of the floor surface. During the winter, the current operation of the hydronic system is regulated by a PID controller. The controller maintains the glycol input temperature that enters the hydronic system at a temperature of $105^{\circ} \mathrm{F}$. This temperature is obtained by regulating the mixing valve 
between the fresh hot glycol from the CHP with the return cold glycol that leaves the hydronic loops. With this input temperature, normally the floor surface would reach a maximum temperature of $85^{0} \mathrm{~F}$, which is the comfortable operating temperature of a radiant floor heating system. Various temperature monitoring and flow control devices were installed at appropriate locations in the hotel during its construction and is illustrated by Figure 16 through 18. Note that the temperature sensors are marked as plus (+) signs. These are connected to the Test Facility through a control and instrumentation cable run.

As for domestic water heating, there are two water tanks with a capacity of 1,000 gallons each. Each tank is equipped with a heat exchanger that is utilized to heat the water by exchanging the thermal energy from the hot glycol. In addition, a gas-fired boiler was installed at each tank and is operated on a stand-by mode. The operation of the domestic water heater is also governed by a PID controller which maintains the water temperature of each tank between $135^{\circ} \mathrm{F}$ to $145^{\circ} \mathrm{F}$. This temperature is achieved by regulating the flow rate of the hot glycol that flows into the heat exchanger of the domestic water heater while the water flow rate is kept constant. During the peak load period, such as the morning, if the temperature of water tank falls below $135^{\circ} \mathrm{F}$ and hot glycol flow is already at the maximum capacity, a stand-by boiler will start to operate, adding needed heat to bring up the water temperature to $135^{0} \mathrm{~F}$. When the temperature of the water tank reaches the prescribed temperature, the glycol and water flow rate is stopped by turning off the pump and closing the valve.

Design concepts for CHP systems based strictly on electric usage is called electric priority mode, and attempts to use heat simply to improve efficiency. A design option 
based solely on the heat usage is called thermal priority model, and considers the electricity as a corollary bonus. Neither of these approaches provides the full benefit of the CHP system. However, for the purpose of this study, the initial control design was based on these two approaches. The initial control system that was utilized to regulate the BCHP operation revolves around PID control. During the thermal priority mode, the PID controller takes a measurement of the main loop at the return side, that is, the cold glycol that is coming from the building, and maintains this temperature to be at $175^{\circ} \mathrm{F}$ by ramping up and down the micoturbine set point in generating the electricity. Under this scheme, there is no exhaust air being released to the atmosphere. During the electric priority mode, as the microturbine generates electricity according to the assigned capacity, a PID controller is programmed to maintain the exhaust air temperature at $185^{\circ} \mathrm{F}$ by varying the opening of the aerator valve, and thus releasing excess hot air to the atmosphere. Thus, the current set up is not operating efficiently.

The operation of BCHP tends to waste energy due to a mismatch between energy supply and demand. For example, when the building thermal demand is actually at lower capacity, oversupply during this period is most likely to occur. Furthermore, the present control system is also lacking of access to local utility rate information where the unit is located. This is important especially when a CHP is operated in the area where time of use utility rate is applied. With time of use type of rate, where different price of energy occurs at different times of the day, analysis needs to be performed in real time to justify the time and the type of operation of the BCHP. For example, when the analysis shows that it costs more to operate the BCHP than buying the same amount of energy through the utility company, then the controller will command the CHP to shutdown the operation 
until the economic savings can start to occur again. Moreover, depending on the agreement with the utility company, the control system with access to utility rate information would be able to determine the time to sell the electricity which is produced through its DG to the utility in order to maintain the overall efficiency.

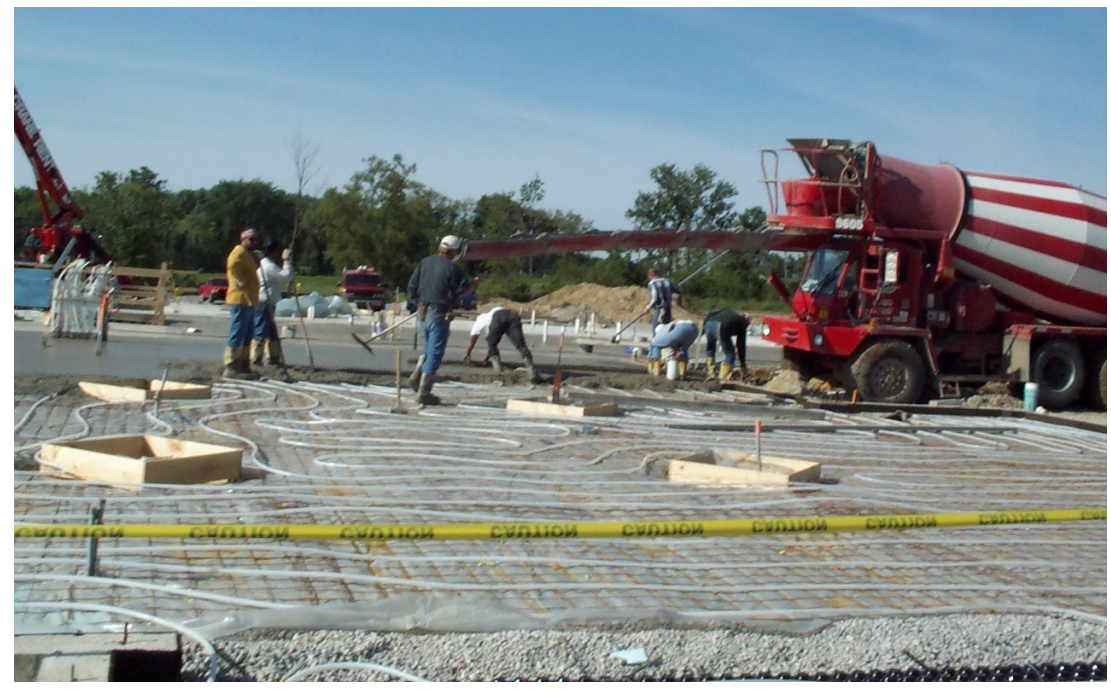

Figure 14: Hydronic Radiant Floor Heating System Installation

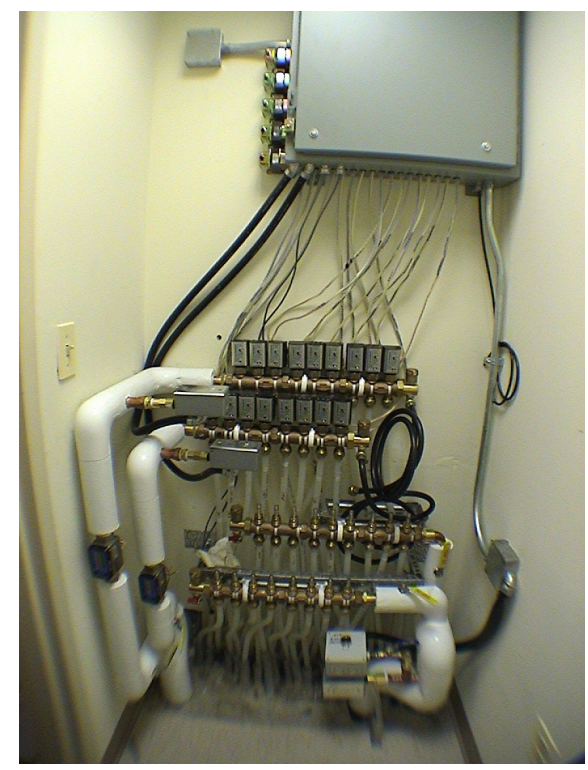

Figure 15: Hydronic Heating Manifold and Control Valves 
POOL MECHANICAL ROOM

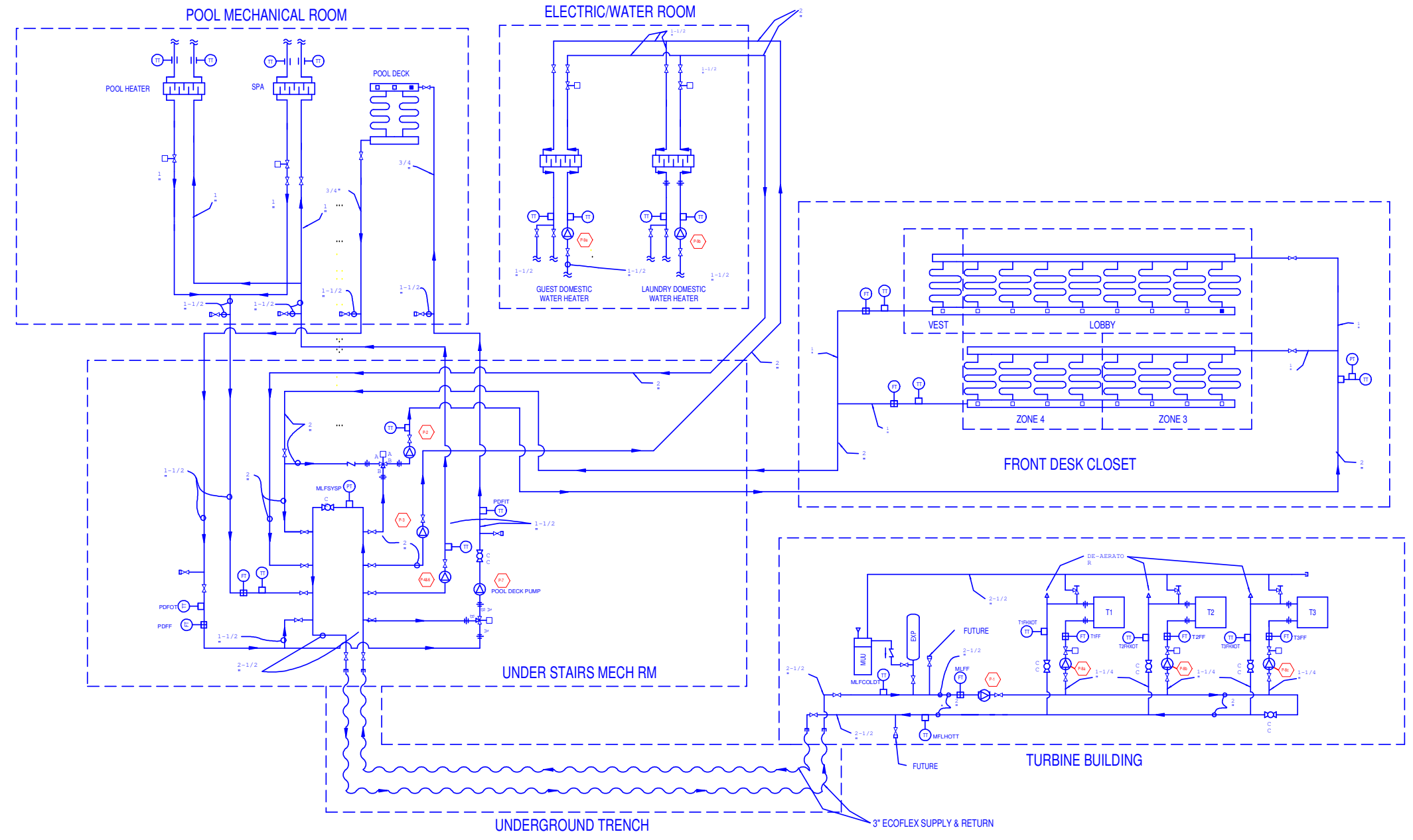

Figure 16: Schematic of BCHP System 


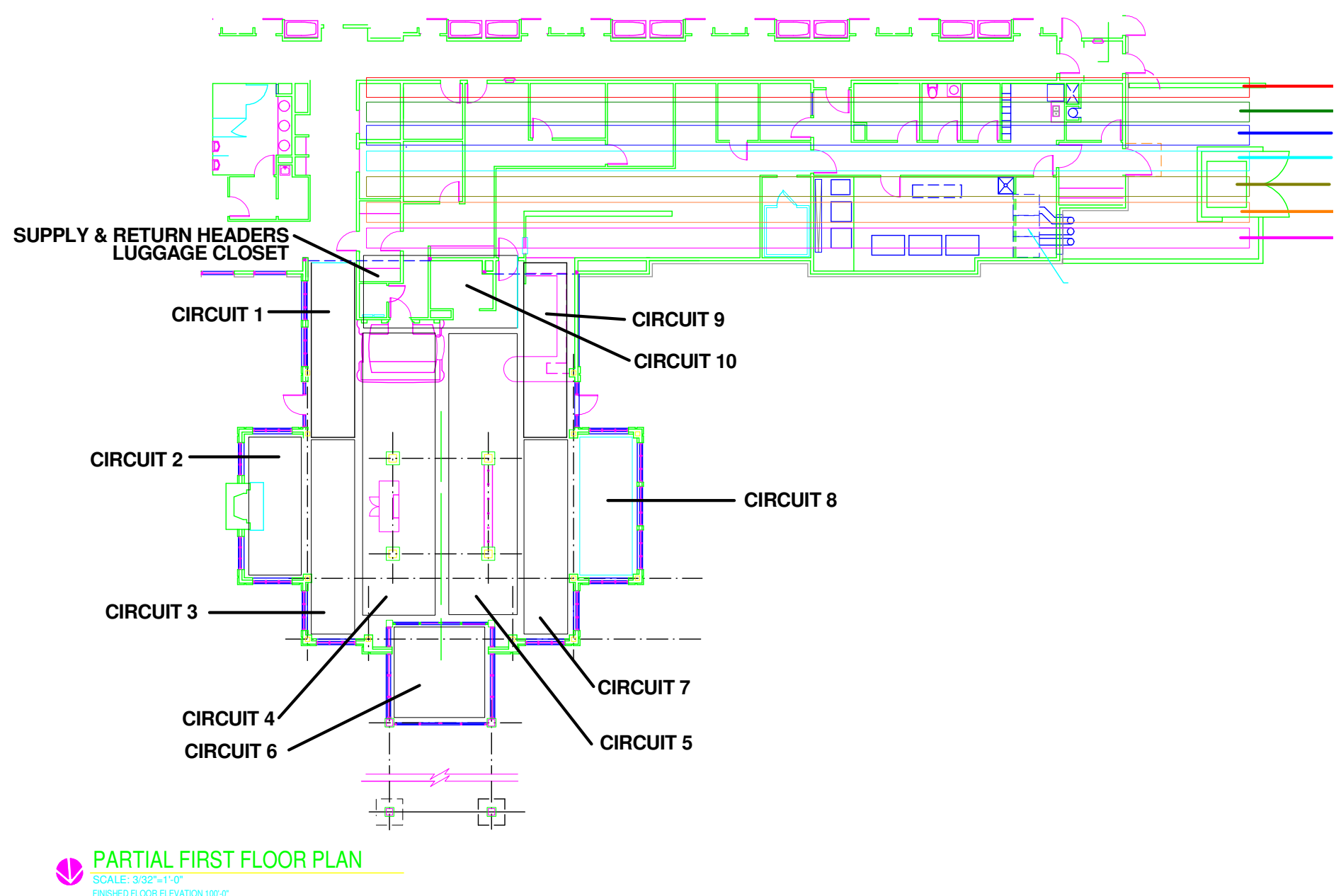

Figure 17: Hydronic Radiant Floor Heating System Circuits 


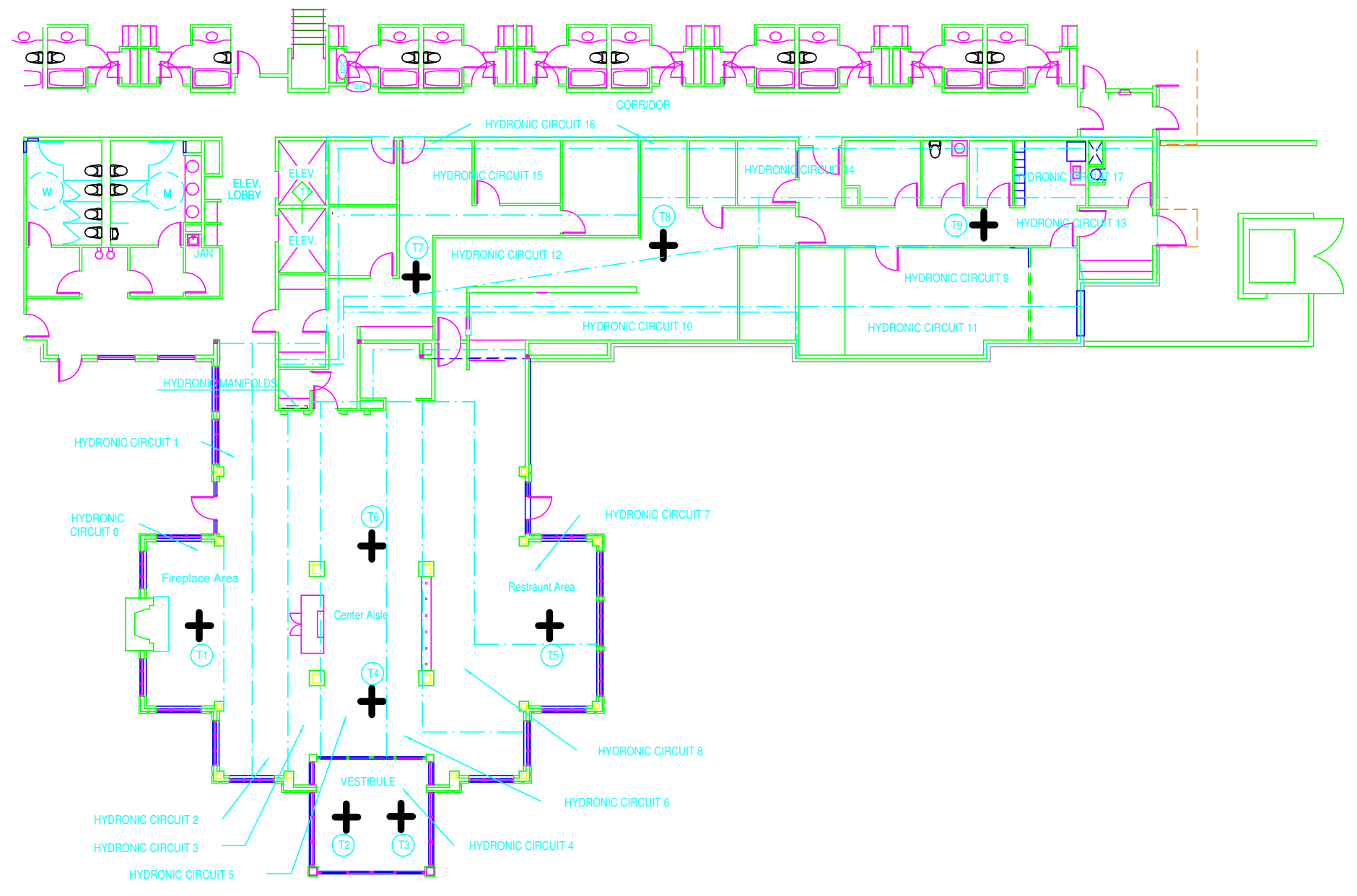

Figure 18: Hydronic Temperature Sensor Location 


\subsection{Nonlinear Thermal Dynamic Model of BCHP System}

To obtain the overall BCHP system model, each of the subsystem models previously defined are interconnected in such a way that they represent the true BCHP system. In order to better track the transformation into a large-scale system, Figure 4.9 depicts the block diagram of the BCHP system, which describes the integration of the previously defined subsystems. Furthermore, in this thesis, only nonlinear systems are considered. As it can be observed from the figure, as a result of being interconnected, subsystem's output may become another subsystem's input. Therefore, Table 1-3 is used to describe the transformation of the notation that is used at the subsystem level to the state notation that is used to model the large-scale system. The number of states for the BCHP system is now 14; the number of controllable input variables is 12 and the constant input variable is 4 . The current set up for the BCHP system is that all states are fully observable. This means there is a sensor measuring the temperature at each state. Hence, the number of output is also 14. The detail transformation of the mathematical equation that integrates the subsystem into a large-scale system can be seen in Appendix B.

The next step is to transform the large-scale system mathematical equation into a nonlinear state space form. In this case, the state space of BCHP follows Equation 3.13, which is a special form of a nonlinear system, input-affine:

$$
\Sigma= \begin{cases}\dot{x}(t)=\tilde{f}(x(t), u(t))=g_{0}(x(t))+\sum_{i=1}^{m} g_{i}(x(t)) u_{i}(t) & ;(\text { State }- \text { Equation }) \\ y(t)=\tilde{h}(x(t))=h(x(t)) & ;(\text { Output }- \text { Equation })\end{cases}
$$


where $x, u$, and $y$ is state, input, and output vector, respectively; and $g_{i}: \Re^{n} \mapsto \Re^{n}$ for $i=0,1, \ldots, m, h: \mathfrak{R}^{n} \mapsto \mathfrak{R}^{p}$ the smooth nonlinear mappings with system dimension $n$ of 14 , number of inputs $m$ of 12 and number of outputs $p$ of 14 . One simplified notation is made to shorten the state space form, that is:

$k_{i}=\frac{U_{i, j} A_{i, j}}{c_{p_{i}} \rho_{i} V_{i}}$

where $i, j=0,1, \ldots, n$, the conductive coefficient between state $i$ and state $j$.

Table 1: State Notation

\begin{tabular}{|l|c|c|}
\hline \multicolumn{1}{|c|}{ Definition } & Subsystem Level & Large Scale \\
& & System Level \\
\hline Microturbine-HX Air Supply temperature & $T_{h o 1}$ & $x_{1}$ \\
\hline Microturbine-HX Glycol Supply temperature & $T_{c o l}$ & $x_{2}$ \\
\hline Main Loop-HX Primary Glycol Supply temperature & $T_{h m x 4}$ & $x_{3}$ \\
\hline Main Loop-HX Primary Glycol Return temperature & $T_{c 4}$ & $x_{4}$ \\
\hline Main Loop Glycol Secondary Supply temperature & $T_{h m l 4}$ & $x_{5}$ \\
\hline Main Loop Glycol Secondary Return temperature & $T_{c m l 4}$ & $x_{6}$ \\
\hline DWH-HX “A” - Glycol Supply temperature & $T_{h o 2 A}$ & $x_{7}$ \\
\hline DWH-HX “A” - Water Return temperature & $T_{c o 2 A}$ & $x_{8}$ \\
\hline DWH-HX “B” - Glycol Supply temperature & $T_{h o 2 B}$ & $x_{9}$ \\
\hline DWH-HX “B” - Water Return temperature & $T_{c o 2 B}$ & $x_{10}$ \\
\hline Hydronic - Glycol Supply temperature & $T_{h m x 3}$ & $x_{11}$ \\
\hline Hydronic - Glycol Return temperature & $T_{h o 3}$ & $x_{12}$ \\
\hline Floor temperature & $T_{z 3}$ & $x_{13}$ \\
\hline Building Space Air temperature & $x_{14}$ \\
\hline
\end{tabular}


Table 2: Constant Input

\begin{tabular}{|l|c|}
\hline \multicolumn{1}{|c|}{ Definition } & Large-Scale System Notation \\
\hline Microturbine-HX air inlet temperature & $b_{1}$ \\
\hline DWH-HX "A" water inlet temperature & $b_{2}$ \\
\hline DWH-HX "B" water inlet temperature & $b_{3}$ \\
\hline HVAC air supply temperature & $b_{4}$ \\
\hline
\end{tabular}

Table 3: Controllable Input

\begin{tabular}{|l|c|}
\hline \multicolumn{1}{|c|}{ Definition } & Large-Scale System Notation \\
\hline Microturbine-HX exhaust air volumetric flow rate & $u_{1}$ \\
\hline Microturbine-HX glycol volumetric flow rate & $u_{2}$ \\
\hline Main loop glycol bypass volumetric flow rate & $u_{3}$ \\
\hline Main loop glycol volumetric flow rate & $u_{4}$ \\
\hline Hydronic glycol volumetric flow rate & $u_{5}$ \\
\hline HVAC supply air volumetric flow rate & $u_{6}$ \\
\hline DWH-HX "A"glycol volumetric flow rate & $u_{7}$ \\
\hline DWH-HX "A" water volumetric flow rate & $u_{8}$ \\
\hline DWH-HX "B" glycol volumetric flow rate & $u_{9}$ \\
\hline DWH-HX "B" water volumetric flow rate & $u_{10}$ \\
\hline Hydronic glycol mixed volumetric flow rate & $u_{11}$ \\
\hline Hydronic glycol return volumetric flow rate & $u_{12}$ \\
\hline
\end{tabular}


LARGE SCALE SYSTEM OF A COMMERCIAL BUILDING THROUGH A SOLID MEDIUM

CONVECTION THROUGH AIR

CONVECTION THROUGH GLYCOL

CONVECTION THROUGH

WATER

MEASURED DATA
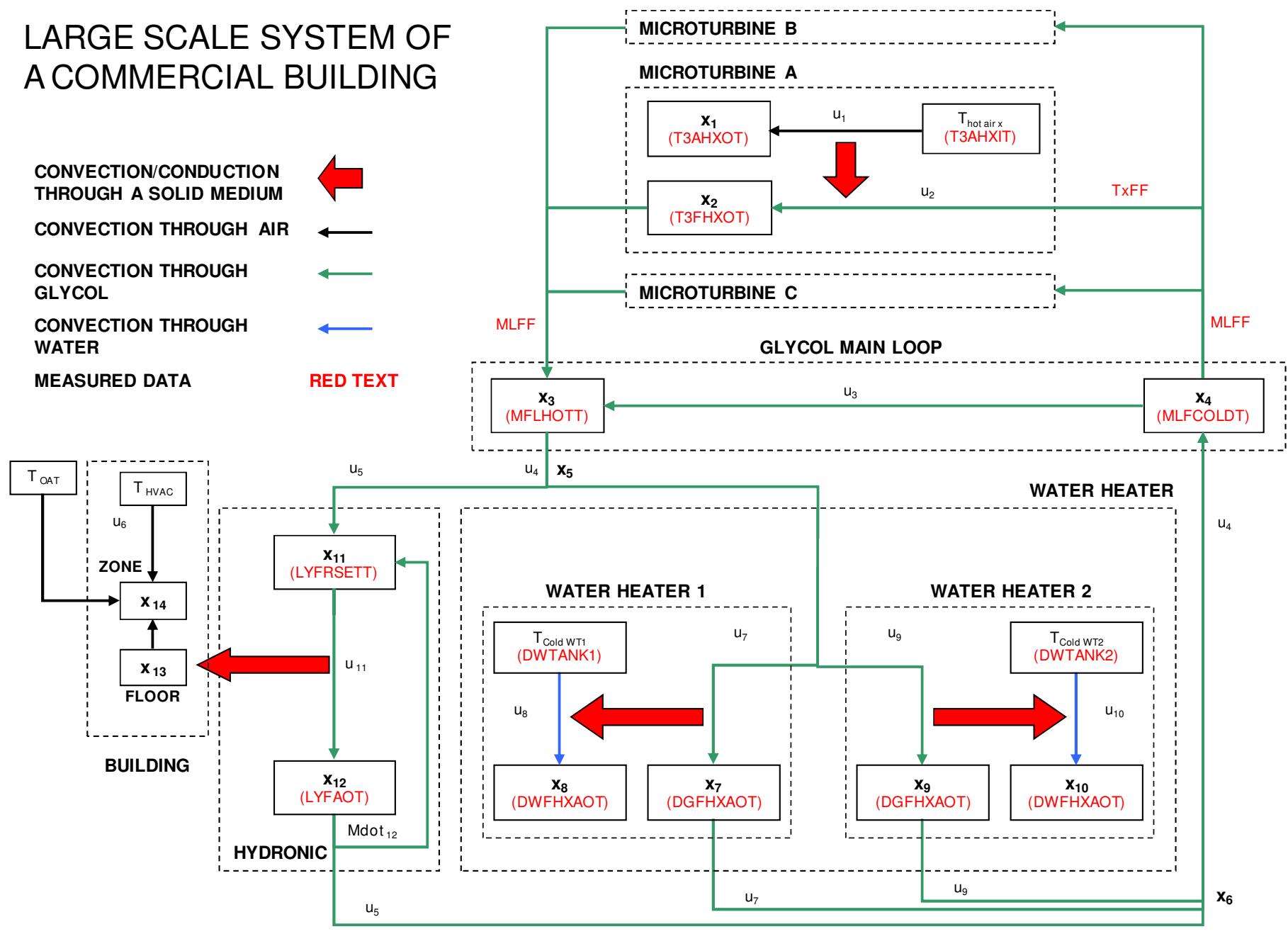

Figure 19: BCHP System and Subsystem Boundary 


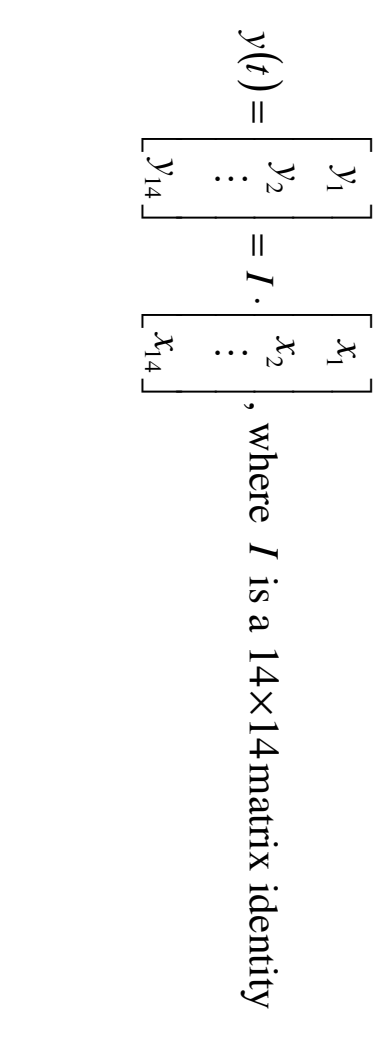

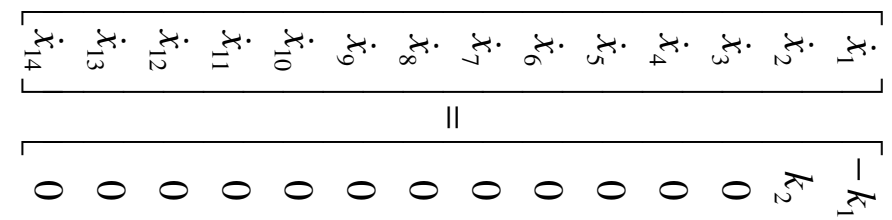

$000000000000 \frac{1}{1}=$

00000000000000

00000000000000

- 0000000000000

00000000000000

000000 m $^{\pi} \pi^{\pi} 000000$

$000000 \frac{1}{0^{\pi}}$ i 000000

$0000 \frac{0}{\pi} \frac{1}{\pi} 00000000$

$0000 \frac{0^{\pi}}{\pi} 00000000$

00000000000000

00000000000000

$x^{\pi} \frac{1}{\pi^{2}} 000000000000$

$\frac{1}{\pi} \pi^{\pi} 000000000000$

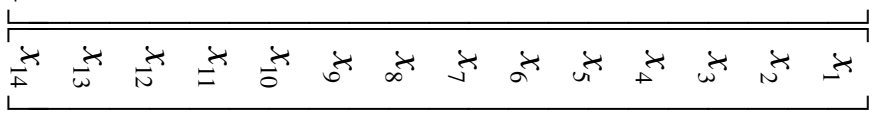


Subsequently, the BCHP state space model of Equation 4.25 is transformed into a more meaningful form where its state equation represents the heat transfer mechanism. The new structure of state equation follows Equation 3.16:

$$
\begin{aligned}
& \dot{x}=A^{0} x+\left(\sum_{i=1}^{m} A^{i} x+B^{i} b\right) u_{i} \\
& y=C x
\end{aligned}
$$

where the following definitions are applied:

4. $A^{0}:=n \times n$ matrix of Linear state transfer term or drift term/conduction:

5. $A^{m}:=n \times n$ matrix of Bilinear state convection term

6. $B^{m}:=n \times n$ Linear input term originating from the input convection

Using Definition 3.4.2 of a Signed Structured Nonlinear Thermal Dynamic System with Drift that is outlined in chapter three, a structured model of BCHP can be obtained.

\section{Definition 3.4.2 (Signed Structured Nonlinear Thermal Dynamic System with Drift):}

The elements of a structure matrix $\left\{A^{k}, B^{k}, C\right\}$ are either fixed at zero or indeterminate values which are assumed to be independent of one another.

$$
\begin{gathered}
\left\{A^{k}\right\}_{i, j}=\left\{\begin{array}{lll}
0 & \text { if } & A_{i j}^{k}=0, \\
+e_{i, j}^{k} & \text { if } & A_{i j}^{k}>0, \\
-e_{i, j}^{k} & \text { if } & A_{i j}^{k}<0,
\end{array}\right. \\
\left\{B^{k^{\prime}}\right\}_{i, j}=\left\{\begin{array}{lll}
0 & \text { if } & B_{i j}^{k}=0, \\
+e_{i, j}^{b^{m}} & \text { if } & B_{i j}^{k}>0, \\
-e_{i, j}^{b^{m}} & \text { if } & B_{i j}^{k}<0,
\end{array}\right.
\end{gathered}
$$




$$
\{C\}_{i, j}=\left\{\begin{array}{lll}
0 & \text { if } & C_{i j}=0, \\
+e_{i, j}^{c} & \text { if } & C_{i j}>0, \\
-e_{i, j}^{c} & \text { if } & C_{i j}<0,
\end{array}\right.
$$

The result of derivation for the structured model of BCHP can be seen in Appendix A. The contribution of the signed structured model in controllability and observability analysis can be observed in chapter five.

Following the structured model, a digraph of BCHP can be constructed utilizing the nonlinear method for developing the digraph. The graph $G=(V, E)_{\text {of }}$ a structured bilinear system represented by its state space model of Equation 3.16 is defined by a vertex set $V$ and an edge set $E$. The vertex set $V$ is given by $B \cup X \cup Y$ with $B=\left\{b_{1}^{1}, \ldots, b_{n}^{m}\right\}$ the set of fixed- input vertices associated with state vertex $i$ and input variable $m, X=\left\{x_{1}, \ldots, x_{n}\right\}$ the set of state vertices, $Y=\left\{y_{1}, \ldots, y_{p}\right\}$ the set of output vertices. Denoting $\left(v, v^{\prime}\right)$ for a directed edge from the initial vertex $v \in V$ to the terminal vertex $v^{\prime} \in V$, the edge set $E$ is described is described by $E_{A^{l}} \cup E_{B^{l}} \cup E_{C}$ with $\left.E_{A^{l}}=\left\{\left(x_{j}, x_{i}\right)\right)\left[A^{l}\right]_{i, j} \neq 0\right\} \quad l=0,1, \ldots, m, \quad E_{B^{\prime}}=\left\{\left(u_{j}, x_{i}\right)\left[B^{l^{\prime}}\right]_{i, j} \neq 0\right\} \quad$ for $\quad l^{\prime}=1, \ldots, m$ and $E_{C}=\left\{\left(x_{j}, y_{i}\right)[C]_{i, j} \neq 0\right\}$. In the latter, for instance $\left[A^{l}\right]_{i, j} \neq 0$ means that the $(i, j)$ thentry of the matrix $\left[A^{l}\right\rfloor$ is a nonzero parameter. Moreover, for $l=0,1, \ldots, m$ and $l^{\prime}=1, \ldots, m$, an index $l$ is assigned to each edge $e \in E_{A^{\prime}} \cup E_{B^{\prime}}$. Note that several indexes may be given to an edge $e$ if it belongs to several subsets $\left(E_{A^{l}} \cup E_{B^{l}}\right)$-edges. For $l=l^{\prime}=1, \ldots, m$, this index correspond to system input $u_{l}$. Figure 20 depicts the BCHP system digraph. 


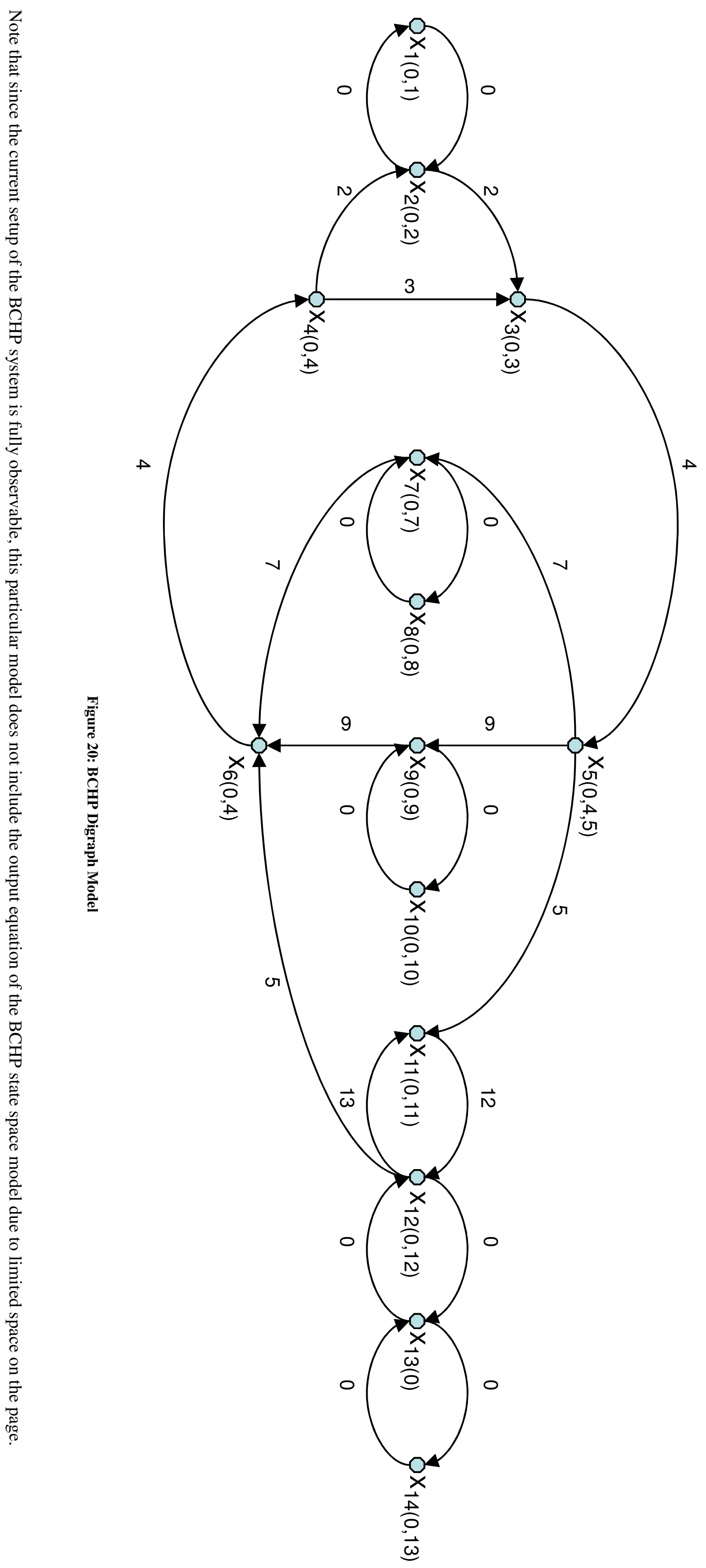




\section{Chapter Five}

\section{CONTROLLABILITY \\ OF A STRUCTURED NONLINEAR THERMAL DYNAMIC SYSTEM VIA CONNECTABILITY APPROACH}

This chapter presents a new approach to controllability of structured nonlinear systems using a graph-theoretic approach. On the basis of a digraph representation, the necessary and sufficient conditions for the controllability of a structured non-linear system are expressed in graphic terms. These conditions have an intuitive interpretation and are easy to check by hand for small systems and by means of well-known combinatorial techniques for large-scale systems. The results presented here then serve as the analytic foundation for controllability analysis for the research system presented in the previous chapter.

\subsection{Controllability of a Non-Linear Thermal Dynamic System}

In this thesis, the following special form of a nonlinear system of input-affine is considered: 
$\Sigma= \begin{cases}\dot{x}(t)=g_{0}(x(t))+\sum_{i=1}^{m} g_{i}(x(t)) u_{i}(t) & ;(\text { State }- \text { Equation }) \\ y(t)=h(x(t)) & ;(\text { Output }- \text { Equation })\end{cases}$

where $x(t)=\left(x_{1}(t), \ldots, x_{n}(t)\right)^{T} \in M \subset \Re^{n}, u(t)=\left(u_{1}(t), \ldots, u_{m}(t)\right)^{T} \in \Omega \subset \Re^{m}$, $y(t)=\left(y_{1}(t), \ldots, y_{p}(t)\right)^{T} \in \mathfrak{R}^{p}$ are, the state, the input and the output vectors, respectively;

and $g_{i}: \mathfrak{R}^{n} \mapsto \mathfrak{R}^{n}$ for $i=0,1, \ldots, m$, and $h: \mathfrak{R}^{n} \mapsto \mathfrak{R}^{p}$ are the smooth nonlinear mappings. As the focus of this research is on a thermal dynamic system, Equation 5.1 that follows the first principle of thermodynamics in energy conservation is considered and is characterized by the bilinear system form described by Equation 5.2.

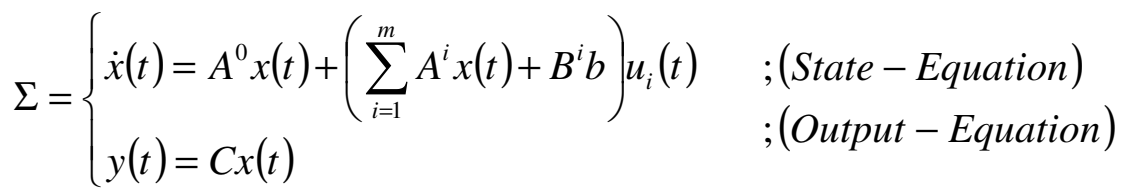

For $i=0, \ldots, m, A^{i} \in \mathfrak{R}^{n \times n}, B^{i} b \in \mathfrak{R}^{n \times 1}$, and $C \in \mathfrak{R}^{p \times n}$ are state space matrices form of $C^{\infty}$ function vector fields on $M$ that represents the following heat transfer mechanisms:

1. $A^{0}:=n \times n$ matrix of Linear state transfer term or drift term/conduction:

2. $A^{i}:=n \times n$ matrix of Bilinear state convection term

3. $B^{i} b:=n \times m$ Linear input term originating from the input convection

4. $C:=p \times n$ matrix of system output

Hereafter a nonlinear thermal dynamic system $\Sigma$ is denoted by bilinear thermal dynamic system (BTS).

The problem statement of controllability in the bilinear thermal dynamic system (BTS) case remains characterized by the set of states that are reachable from a given initial state as defined previously in chapter two: 


\section{Definition 2.3.1 $(U-$ reachable $)$ :}

Given a subset $U \subseteq M, x^{1}$ is $U$-reachablefrom $x^{0}$ (denoted by $\left.x^{1} R_{U} x^{0}\right)$ if there exists a bounded measurable control $\left(u(t),\left[t^{0}, t^{1}\right]\right)$ satisfying $u(t) \in \Omega$ for $t \in\left[t^{0}, t^{1}\right]$ such that the corresponding solution $\left(x(t),\left[t^{0}, t^{1}\right]\right)$ of differential equation (2) satisfies $x\left(t^{0}\right)=x^{0}$, $x\left(t^{1}\right)=x^{1}$ and $x(t) \in U$ for all $t \in\left[t^{0}, t^{1}\right]$. Denote $R\left(x^{0}\right)=\left\{x^{1} \in M: x^{1} R x^{0}\right\}$ the set of points reachable from $x^{0}$.

Furthermore, previously defined local state controllability is also considered:

\section{Definition 2.3.3 (Local State Controllability):}

The system $\Sigma$ is said to be locally controllable at $x^{0}$ if for every neighborhood $U$ of $x^{0}$, $R_{U}\left(x^{0}\right)$ is also a neighborhood of $x^{0} ; \Sigma$ is locally controllable if it is locally controllable at every $x \in M$.

Moreover, in order to test the controllability of a nonlinear system, the previously defined theorem of the necessary and sufficient condition for a nonlinear system to be locally controllable is utilized.

\section{Theorem 2.3.1 (Controllability Rank Test):}

A nonlinear system $\Sigma$ satisfies the controllability rank condition at $x^{0}$ if in a neighborhood of $x^{0}, \operatorname{dim}\left[\Delta_{C}\right]=n$. If this holds for all $x^{0} \in M, \Sigma$ satisfies the controllability rank condition. Thus, if $\Sigma$ satisfies the controllability rank condition at $x^{0} \in M$, then $\Sigma$ has the local reachability property at $x^{0}$

Therefore, the first step toward the analysis of the local controllability of a nonlinear system is to find $R$, which is established through the derivation of 
controllability distribution $\Delta_{\mathrm{C}}$ using Lie bracket. Isidori $[1995,1]$ proposes an algorithm for constructing the controllability distributions as follows:

\section{Algorithm for Constructing the Controllability Distribution}

1. Starting Point: $\Delta_{0}=\operatorname{span}\left\{g_{1}, \ldots, g_{m}\right\}$

2. Development of the controllability Distribution: $\Delta_{k}=\Delta_{k-1}+\sum_{i=0}^{m}\left[g_{i}, \Delta_{k-1}\right]$

Note that one term in the last sum $\left[g_{i}, \Delta_{k-1}\right]$ is computed by using the functions

$\left(\phi_{1}, \ldots, \phi_{l}\right)$ spanning the distribution $\Delta_{\mathrm{k}-1}:\left[g_{i}, \Delta_{k-1}\right]=\operatorname{span}\left\{\left[g_{1}, \phi_{1}\right], \ldots,\left[g_{1}, \phi_{l}\right]\right\}$

3. Stopping Condition: If $\exists k^{*}$ such that $\Delta_{k}=\Delta_{k-1}$, then $\Delta_{\mathrm{C}}=\Delta_{k^{*}}=\left\langle g_{0}, \ldots, g_{m} \mid \Delta_{0}\right\rangle$ where $g_{0}=A^{0} x$ and $g_{i}=\left(A^{i} x+B^{i} b\right)$

\subsection{Controllability of a Structured Non-Linear Thermal Dynamic System}

When the exact knowledge of the state space matrices characterizing the system's model is not available, a structured model approach is suggested. In structured modeling, the system is characterized by system matrices that preserve the zero/non-zero entries in the state space matrices determined by the physical laws, which in this case is thermodynamic. Thus, the structured model is defined as a model where the fixed zeros are conserved while the non-zeros are replaced by free parameters. In the case of this research, a signed structured nonlinear thermal dynamic system as previously defined in chapter 3 is considered. 


\section{Definition 3.4.2 (Signed Structured Nonlinear Thermal Dynamic System with Drift):}

The elements of a structure matrix $\left\{A^{k}, B^{k^{\prime}}, C\right\}$ are either fixed at zero or indeterminate values which are assumed to be independent of one another.

$$
\begin{gathered}
\left\{A^{k}\right\}_{i, j}=\left\{\begin{array}{lll}
0 & \text { if } & A_{i j}^{k}=0, \\
+e_{i, j}^{k} & \text { if } & A_{i j}^{k}>0, \\
-e_{i, j}^{k} & \text { if } & A_{i j}^{k}<0,
\end{array}\right. \\
\left\{B^{k^{\prime}}\right\}_{i, j}=\left\{\begin{array}{lll}
0 & \text { if } & B_{i j}^{k}=0, \\
+e_{i, j}^{b^{m}} & \text { if } & B_{i j}^{k}>0, \\
-e_{i, j}^{b^{m}} & \text { if } & B_{i j}^{k}<0,
\end{array}\right. \\
\{C\}_{i, j}=\left\{\begin{array}{lll}
0 & \text { if } \quad C_{i j}=0, \\
+e_{i, j}^{c} & \text { if } \quad C_{i j}>0, \\
-e_{i, j}^{c} & \text { if } & C_{i j}<0,
\end{array}\right.
\end{gathered}
$$

Hereafter, a structured nonlinear thermal dynamic system is denoted by a structured bilinear thermal dynamic system (SBTS).

Note that in the case of structured thermal dynamic system models which follow the form of Equation 5.2, the following equalities characterized the SBTS:

1. At node $i, e_{i, i}^{0}=-e_{i, j}^{0}$, for $j=1, \ldots, n$, except $i=j$

2. At node $i, e_{i, j}^{k}=-e_{i, j}^{b^{k}}$ for $j=1, \ldots, n$, except $i=j$ and $k=1, \ldots, m$

where $n$ is the dimension of state space model and $m$ is the number of input variables.

Therefore, when the concern in the controllability analysis is in the form of structured matrices, structural controllability is applied to ensure any given structured system is controllable. Hence, equipped by Definition 2.4.3 to 2.4.5 from chapter two, the following definition for controllability of a structured nonlinear system can be devised. 


\section{Definition 5.2.1 (Structural Controllability for a Nonlinear System):}

A class of nonlinear systems given by their structure matrix pairs $\left[A^{0}, A^{i}, B^{i}\right]$ for $i=1, \ldots, m$ is said to be structurally controllable (for short, s-controllable) if there exists at least one admissible realization $\left(A^{0}, A^{i}, B^{i}\right) \in\left[A^{0}, A^{i}, B^{i}\right]$ being controllable in the usual numerical sense.

As a result, based on Theorem 2.3.1 of controllability rank test for a nonlinear system, the necessary and sufficient condition for the controllability of a structured nonlinear system becomes the following:

\section{Theorem 5.2.1 (Controllability Rank Test for a Structured Nonlinear System):}

A structured nonlinear system $[\Sigma]$ characterized by structure matrix pair $\left[A^{0}, A^{i}, B^{i}\right]$ for $i=1, \ldots, m$ is structurally locally controllable if, for almost all the realization of $(\Sigma) \in[\Sigma]$, there exists controllability distribution $\left(\Delta_{C}\right) \in\left[\Delta_{C}\right]$ of structural dimension $n$.

Note that structural dimension here is equivalent to structural rank of Definition 2.4.5.

\subsection{Controllability of a Structured Non-Linear Thermal Dynamic System via Connectability Approach}

This section focuses on providing the graphic condition equivalent to the one of Theorem 5.3.1 on structural controllability rank condition for a structured nonlinear system. Since the focus of the research is placed upon a bilinear thermal dynamic system (BTS), the first part of this section is devoted to some definition of a digraph utilized as the tool for analyzing structural controllability. The second part discusses the proposed 
graphical criterion that characterized the structural controllability of a structured bilinear thermal dynamic system (SBTS).

\subsubsection{Digraph Definition for a Structured Thermal Dynamic System}

Given a structured bilinear thermal dynamic system ${ }^{[\Sigma]}$, some definitions based on digraph theory described in chapter three are devised:

\section{Definition 5.3.1 (State Digraph):}

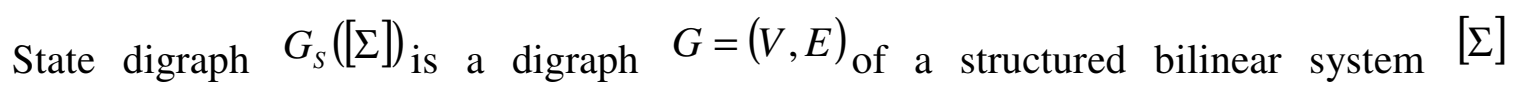
represented by its state space model of equation 5.2, and is characterized by the followings:

a. The vertex set $V$ is given by $B \cup X$ with $B=\left\{b_{1}^{1}, \ldots, b_{n}^{m}\right\}_{\text {the set of fixed- input }}$ vertices associated with state vertex $i_{\text {and input variable } m \text {, and }}$ $X=\left\{x_{1}, \ldots, x_{n}\right\}_{\text {the set of state vertices. }}$

b. The edge set $E$ is described by $E_{A^{l}} \cup E_{B^{l^{l}} \text { with }} E_{A^{l}}=\left\{\left(x_{j}, x_{i}\right)\left[A^{l}\right]_{i, j} \neq 0\right\}$ $l=0,1, \ldots, m$, and $E_{B^{l^{\prime}}}=\left\{\left(u_{j}, x_{i}\right)\left[B^{l^{\prime}}\right]_{i, j} \neq 0\right\}$ for $l^{\prime}=1, \ldots, m$.

For $l=0,1, \ldots, m$ and $l^{\prime}=1, \ldots, m$, an index $l$ is assigned to each edge $e \in E_{A^{l}} \cup E_{B^{l^{\prime}}}$. Note that several indexes may be given to an edge $e$ if it belongs to several subsets $\left(E_{A^{l}} \cup E_{B^{l^{\prime}}}\right)-$ edges . For $l=l^{\prime}=1, \ldots, m$, this index corresponds to system input $u_{l}$. 


\section{Definition 5.3.2 (Input Connectable):}

State digraph $G_{S}([\Sigma])$ is input connectable if there exists path from at least $m$ distinct input edges to every state vertex.

\subsubsection{Main Results}

An obvious precondition of controllability is that the system inputs are able to influence all state variables. Said in graph-theoretic terms for bilinear systems, there must exist paths from input edges to all state vertices. Therefore, the following proposition is suggested in this research:

\section{Proposition 5.3.1:}

A structured bilinear thermal dynamic system (SBTS) $[\Sigma]$ is structurally controllable if and only if in its associated state diagraph $G_{S}([\Sigma])$ is input connectable.

In order to proof Proposition 5.4.1, it is important to show an SBTS that is inputconnectable, possesses the ability to satisfy the necessary and sufficient conditions for controllability in the standard numerical sense of Lie algebra rank condition as described in Theorem 5.3.1. Thus, the following lemma is formulated:

\section{Lemma 5.3.1:}

If an $n$-order SBTS $[\Sigma]$ characterized by structure matrix $\operatorname{set}\left[A^{0}, A^{i}, B^{i}\right]$ for $i=1, \ldots, m$ is input connectable, then there holds a structured matrix formed by controllability distribution $\left[\Delta_{C}\right]$ of $[\Sigma]$ with structural rank $n$ for almost all the realization of $(\Sigma) \in[\Sigma]$. 


\section{Proof of Lemma 5.3.1}

In order to verify Lemma 5.3.1, $n$-order SBTS is studied gradually to find the characteristics that would guarantee a result which satisfies the Lie algebra rank condition. It will be shown that in order to obtain a structured controllability distribution $\left[\Delta_{C}\right]$ of $[\Sigma]$ with structural rank $n$ for almost all the realization of $(\Sigma) \in[\Sigma]$, a connectability property called input connectability must exist.

First, consider an admissible second order bilinear thermal dynamic system following the thermodynamic first principle $(n=2)$ with single input $(m=1)$ described by the following state Equation:

$\Sigma: \dot{x}=\left[\begin{array}{cc}-k s_{1} & k s_{1} \\ k s_{2} & -k s_{2}\end{array}\right]\left[\begin{array}{l}x_{1} \\ x_{2}\end{array}\right]+\left[\begin{array}{cc}-k 1_{1} & k 1_{1} \\ k 1_{2} & -k 1_{2}\end{array}\right]\left[\begin{array}{l}x_{1} \\ x_{2}\end{array}\right] u_{1}+\left[\begin{array}{l}b 1_{1} \\ b 1_{2}\end{array}\right] u_{1}$

To create a relationship that is proposed by Lemma 5.4.1, a signed structured model $[\Sigma]$ is utilized. Thus, Equation 3.1 now becomes the following state equation:

$[\Sigma]: \dot{x}=\left[\begin{array}{cc}-E 0_{1,1} & E 0_{2,1} \\ E 0_{1,2} & -E 0_{2,2}\end{array}\right]\left[\begin{array}{l}x_{1} \\ x_{2}\end{array}\right]+\left[\begin{array}{cc}-E 1_{1,1} & E 1_{2,1} \\ E 1_{1,2} & -E 1_{2,2}\end{array}\right]\left[\begin{array}{l}x_{1} \\ x_{2}\end{array}\right] u_{1}+\left[\begin{array}{l}b 1_{1} \\ b 1_{2}\end{array}\right] u_{1}$

Given an SBTS described by Equation 5.2, parameter $E k_{i, j}$ represents not only the structured entry of $A_{k}$ matrix of $i$-th column and $j$-th row but also the edge that connects state vertex $i$ to state vertex $j$ associated with matrix $A_{k}$. Furthermore, $b k_{j}$ refers to the entry of $k$-th input of structured vector $B_{k}$ of row $j$-th, which also represents the edge from vertex $b k_{j}$ to state vertex $j$. For the completeness, since SBTS is governed by the first principle of thermodynamics, based on the transformation the following equality is applied: 
1. $E 0_{1,1}=E 0_{2,1}=k s_{1}$

2. $E O_{1,2}=E 0_{2,2}=k s_{2}$

3. $E 1_{1,1}=E 1_{2,1}=E 1_{1}=k 1_{1}$

4. $E 1_{1,1}=E 1_{2,1}=E 1_{2}=k 1_{2}$

Using this SBTS matrix equation, Isidori's algorithm [1995, 1] for structured controllability distribution $\left[\Delta_{C}\right]$ of $[\Sigma]$ is applied and the following structured matrix, consisting of two vectors, is obtained:

$$
\Delta_{c}=\ldots
$$

$\Delta_{1}=\ldots$

$\int\left[\begin{array}{c}-E 1_{11} x_{1}+E 1_{21} x_{2}+B 1_{1} \\ E 1_{12} x_{1}-E 1_{22} x_{2}+B 1_{2}\end{array}\right], \ldots$

$\left.\left\{\left[\begin{array}{l}\left(\left(E 1_{11} E 0_{11}+E 1_{21} E 0_{12}\right)-\left(E 0_{11} E 1_{11}+E 0_{21} E 1_{12}\right)\right) x_{1}+\left(\left(-E 1_{11} E 0_{21}-E 1_{21} E 0_{22}\right)-\left(-E 0_{11} E 1_{21}-E 0_{21} E 1_{22}\right)\right) x_{2} \\ \left(\left(-E 1_{12} E 0_{11}-E 1_{22} E 0_{12}\right)-\left(-E 0_{12} E 1_{11}-E 0_{22} E 1_{12}\right)\right) x_{1}+\left(\left(E 1_{12} E 0_{21}+E 1_{22} E 0_{22}\right)-\left(E 0_{12} E 1_{21}+E 0_{22} E 1_{22}\right)\right) x_{2}\end{array}\right]-\left[\begin{array}{c}-E 0_{11} B 1_{1}+E 0_{21} B 1_{2} \\ E 0_{12} B 1_{1}-E 0_{22} B 1_{2}\end{array}\right]\right]\right\}$

To check the Lie algebra rank condition of the structured matrix $\left\{\Delta_{1}\right\}$, its determinant

$\left(\operatorname{Det}\left[\Delta_{1}\right]\right)$ is subsequently evaluated. Equation 5.6 described the structured parametric equation of $\operatorname{Det}\left(\left[\Delta_{1}\right]\right)$ :

$\left[E 0_{11}\left(E 1_{11} E 1_{12}\right)+E 0_{21}\left(E 1_{12} E 1_{12}\right)-E 0_{12}\left(E 1_{11} E 1_{11}\right)-E 0_{22}\left(E 1_{11} E 1_{12}\right)\right] x_{1}^{2}-\ldots$

$\left[E 0_{11}\left(E 1_{21} E 1_{12}\right)+E 0_{21}\left(E 1_{12} E 1_{22}\right)-E 0_{12}\left(E 1_{11} E 1_{21}\right)-E 0_{22}\left(E 1_{21} E 1_{12}\right)\right] 2 x_{1} x_{2}+\ldots$

$\left[E 0_{11}\left(E 1_{21} E 1_{22}\right)+E 0_{21}\left(E 1_{22} E 1_{22}\right)-E 0_{12}\left(E 1_{21} E 1_{21}\right)-E 0_{22}\left(E 1_{21} E 1_{22}\right)\right] x_{2}{ }^{2}+\ldots$

$\left[-2 E 0_{11}\left(E 1_{12} E 1_{1} b 1_{1}\right)+2 E 0_{21}\left(E 1_{12} E 1_{2} b 1_{2}\right)+\left(2 E 0_{12}\left(E 1_{11} E 1_{1} b 1_{1}\right)-E 0_{12}\left(E 1_{21} E 1_{2} b 1_{2}\right)\right)-E 0_{22}\left(E 1_{11} E 1_{2} b 1_{2}\right)\right] x_{1}-$

$\left[-E 0_{11}\left(E 1_{22} E 1_{1} b 1_{1}\right)-\left(E 0_{21}\left(E 1_{12} E 1_{1} b 1_{1}\right)-2 E 0_{21}\left(E 1_{22} E 1_{2} b 1_{2}\right)\right)+2 E 0_{12}\left(E 1_{21} E 1_{1} b 1_{1}\right)-2 E 0_{22}\left(E 1_{21} E 1_{2} b 1_{2}\right)\right] x_{2}+\ldots$

$\left[-E 0_{11}\left(E 1_{2} b 1_{2} E 1_{1} b 1_{1}\right)+E 0_{21}\left(E 1_{2} E 1_{2} b 1_{2} b 1_{2}\right)-E 0_{12}\left(E 1_{1} E 1_{1} b 1_{1} b 1_{1}\right)+E 0_{22}\left(E 1_{1} E 1_{2} b 1_{1} b 1_{2}\right)\right]$

In order to satisfy Lie algebra rank condition, a nonzero $\operatorname{Det}\left(\left[\Delta_{1}\right]\right)$ is required. Using the structured parametric equation described by Equation 5.6, gradually identifying the set of parameters that would result in nonzero determinant, given any combination of input parameters is performed. Tables 4 and 5 show the result of this determinant approach analysis. Table 4 shows the set parameters of Equation 5.6 that must exist in order to obtain a non-zero $\operatorname{Det}\left(\left[\Delta_{1}\right]\right)$ if system input enters through state $x_{l}$ as described 
by Equation 5.7. Tables 5 summarizes the parameters set of Equation 5.6 that must exist in order to obtain a non-zero $\operatorname{Det}\left(\left[\Delta_{1}\right]\right)$ if system input enters either through state $x_{1}$ or state $x_{2}$ as described by Equation 5.8, or both.

$$
\begin{aligned}
& {[\Sigma]: \dot{x}=\left[\begin{array}{cc}
-E 0_{1,1} & E 0_{2,1} \\
E 0_{1,2} & -E 0_{2,2}
\end{array}\right]\left[\begin{array}{l}
x_{1} \\
x_{2}
\end{array}\right]+\left[\begin{array}{cc}
-E 1_{1,1} & E 1_{2,1} \\
0 & 0
\end{array}\right]\left[\begin{array}{l}
x_{1} \\
x_{2}
\end{array}\right] u_{1}+\left[\begin{array}{c}
b 1_{1} \\
0
\end{array}\right] u_{1}} \\
& {[\Sigma]: \dot{x}=\left[\begin{array}{cc}
-E 0_{1,1} & E 0_{2,1} \\
E 0_{1,2} & -E 0_{2,2}
\end{array}\right]\left[\begin{array}{l}
x_{1} \\
x_{2}
\end{array}\right]+\left[\begin{array}{cc}
0 & 0 \\
E 1_{1,2} & -E 1_{2,2}
\end{array}\right]\left[\begin{array}{l}
x_{1} \\
x_{2}
\end{array}\right] u_{1}+\left[\begin{array}{c}
0 \\
b 1_{2}
\end{array}\right] u_{1}}
\end{aligned}
$$

Table 4: Parametric Equation for Input Vector of state $x_{1}$

\begin{tabular}{|c|c|c|}
\hline Input Vector & & Parameter/Edges \\
$\left(\boldsymbol{g}_{1}\right)$ Parameters & Det $\left(\left[\Delta_{1}\right]\right)$ Parameteric Equation & that must Exist for \\
(Edge/s) & $-E 0_{12}\left(E 1_{11} E 1_{11}\right) x_{1}{ }^{2}$ & Det $\left(\left[\Delta_{1}\right]\right) \neq \mathbf{0}$ \\
\hline \hline$E 1_{11}$ & $-E 0_{12}\left(E 1_{21} E 1_{21}\right) x_{2}{ }^{2}$ & $\left\{E 1_{11}, E 0_{12}\right\}$ \\
\hline$E 1_{21}$ & $-E 0_{12}\left(E 1_{1} b 1_{1} E 1_{1} b 1_{1}\right)$ & $\left\{E 1_{21}, E 0_{12}\right\}$ \\
\hline$E 1_{1} b 1_{1}$ & $-E 0_{12}\left(E 1_{11} E 1_{11}\right) x_{1}{ }^{2}+E 0_{12}\left(E 1_{11} E 1_{21}\right) 2 x_{1} x_{2}-E 0_{12}\left(E 1_{21} E 1_{21}\right) x_{2}{ }^{2}$ & $\left\{E 1 b 1_{1}, E 0_{12}\right\}$ \\
\hline$E 1_{11} \& E 1_{21}$ & $\left.-E 0_{12}\left(E 1_{11} E 1_{11}\right) x_{1}{ }^{2}+2 E 0_{12}\left(E 1_{11} E 1_{1} b 1_{1}\right) x_{1}-E 0_{12}\left(E 1_{1} b 1_{1}\right)^{2}, E 0_{12}\right\}$ \\
\hline$E 1_{11} \& E 1_{1} b 1_{1}$ & $-E 0_{12}\left(E 1_{21} E 1_{21}\right) x_{2}{ }^{2}-2 E 0_{12}\left(E 1_{21} E 1_{1} b 1_{1}\right) x_{2}-E 0_{12}\left(E 1_{1} b 1_{1}\right)^{2}$ & $\left\{E 1_{11} \cup E 1_{1} b 1_{1}, E 0_{12}\right\}$ \\
\hline$E 1_{21} \& E 1_{1} b 1_{1}$ & $-E 0_{12}\left(E 1_{11} E 1_{11}\right) x_{1}{ }^{2}+E 0_{12}\left(E 1_{11} E 1_{21}\right) 2 x_{1} x_{2}-E 0_{12}\left(E 1_{21} E 1_{21}\right) x_{2}{ }^{2}$ & $\left\{E 1_{21} \cup E 1_{1} b 1_{1}, E 0_{12}\right\}$ \\
\hline$E 1_{11}, E 1_{21} \& E 1_{1} b 1_{1}$ & $+2 E 0_{12}\left(E 1_{11} E 1_{1} b 1_{1}\right) x_{1}-2 E 0_{12}\left(E 1_{21} E 1_{1} b 1_{1}\right) x_{2}-E 0_{12}\left(E 1_{1} b 1_{1}\right)^{2}$ & $\left\{E 1_{11} \cup E 1_{21} \cup E 1_{1} b 1_{1}, E 0_{12}\right\}$ \\
\hline
\end{tabular}

Column 1 of Table 4 shows several possible combinations of input vectors $\left(g_{1}\right)$ composed of input parameter/s connect/s only to state $x_{1}$, which is described by Equation 5.7. The application of these different input vector combinations results in several 
different parametric determinant equations as described in column 2 of Table 4. Subsequently, these parametric equations are used to identify parameter(s) that satisfies(y) the Lie algebra rank condition, that is, for the determinant equation to not equal zero. It is observed from each equation that at least one nonzero parameter must exist in addition to the input parameter(s). Given that each parameter also represents an edge that connect initial vertex to terminal vertex, thus, based on this observation, it implies that any combination of input an edge $E 1_{i 1}$ that enters to state $x_{1}$, an additional edge connects that state $x_{1}$, to state vertex 2 , that is $E 0_{12}$, is required. The same approach is performed by assuming that the input vector $\left(g_{1}\right)$ enters the system to state $x_{2}$ as described by Equation 5.8 .

Furthermore, when dealing with a nonlinear type of system, multi equilibrium is one of the properties that comes with it. This property may cause a Lie algebra rank condition of the structured matrix $\Delta_{1}$ to have a rank of less than $n$. Here, using Equation 5.6, the singularity that is obtained when $\operatorname{Det}\left(\left[\Delta_{1}\right]\right)=0$ can now be identified as well. Since it is known that the parameters of Equation 5.6 cannot be zero, $\operatorname{Det}\left(\left[\Delta_{1}\right]\right)=0$ is most likely resulted from the value of the state condition itself. Table 5 summarizes the set parameters of Equation 5.6 that must exist in order to obtain a non-zero $\operatorname{Det}\left(\left[\Delta_{1}\right]\right)=0$ if system input enters either through state $x_{1}$ or state $x_{2}$ as described by Equation 5.7 and 5.8 , or both. This table also includes all possible singularity points that can result in $\operatorname{Det}\left(\left[\Delta_{1}\right]\right)=0$. In addition, $\operatorname{Det}\left(\left[\Delta_{1}\right]\right)=0$ can also be caused from the identicalness parameter value associated with each state. In this case, $\operatorname{Det}\left(\left[\Delta_{1}\right]\right)=0$ is possible to happen when the following identical equations occur: 
1. $\mathrm{ks}_{1}=\mathrm{EO}_{1,1}=E 0_{2,1}=k s_{2}=E 0_{1,2}=E O_{2,2}$

2. $\mathrm{k} 1_{1}=E 1_{1,1}=E 1_{2,1}=E 1_{1}=k 1_{2}=E 1_{1,2}=E 1_{2,2}=E 1_{2}$

Table 5: Edge/s that maintain SBTS structural controllability

\begin{tabular}{|c|c|c|}
\hline Input edge/s & $\begin{array}{l}\text { Additional } \\
\text { Edge/s that } \\
\text { must Exist for } \\
\text { Det }\left[\Delta_{1}\right] \neq \mathbf{0}\end{array}$ & Singular Point \\
\hline$\left(E 1_{11}\right) \vee\left(E 1_{21}\right) \vee\left(E 1_{1} b 1_{1}\right)$ & $E 0_{12}$ & $x_{1}=x_{2}=b 1_{1}=0$ \\
\hline$\left\{\left(E 1_{11}\right) \cup\left(E 1_{21}\right)\right\} \vee\left\{\left(E 1_{11}\right) \cup\left(E 1_{1} b 1_{1}\right)\right\} \vee\left\{\left(E 1_{21}\right) \cup\left(E 1_{1} b 1_{1}\right)\right\}$ & $E 0_{12}$ & $\begin{array}{l}x_{1}=x_{2} \\
x_{1}=b 1_{1} \\
x_{2}=-b 1_{1}\end{array}$ \\
\hline$\left\{\left(E 1_{11}\right) \cup\left(E 1_{21}\right) \cup\left(E 1_{1} b 1_{1}\right)\right\}$ & $E 0_{12}$ & $x_{1}=x_{2}=b 1_{1}$ \\
\hline$\left(E 1_{12}\right) \vee\left(E 1_{22}\right) \vee\left(E 1_{2} b 1_{2}\right)$ & $E 0_{21}$ & $x_{1}=x_{2}=b 1_{1}=0$ \\
\hline$\left\{\left(E 1_{12}\right) \cup\left(E 1_{22}\right)\right\} \vee\left\{\left(E 1_{12}\right) \cup\left(E 1_{2} b 1_{2}\right)\right\} \vee\left\{\left(E 1_{22}\right) \cup\left(E 1_{2} b 1_{2}\right)\right\}$ & $E 0_{21}$ & $\begin{array}{l}x_{1}=x_{2} \\
x_{1}=b 1_{1} \\
x_{2}=-b 1_{1}\end{array}$ \\
\hline$\left\{\left(E 1_{12}\right) \cup\left(E 1_{22}\right) \cup\left(E 1_{2} b 1_{2}\right)\right\}$ & $E 0_{21}$ & $x_{1}=x_{2}=b 1_{2}$ \\
\hline$\left\{\left(E 1_{11}\right) \vee\left(E 1_{21}\right) \vee\left(E 1_{1} b 1_{1}\right)\right\} \cup\left\{\left(E 1_{12}\right) \vee\left(E 1_{22}\right) \vee\left(E 1_{2} b 1_{2}\right)\right\}$ & $E 0_{12} \cup E 0_{21}$ & $x_{1}=x_{2}, x_{1}=b 1_{1}, x_{2}=b 1_{2}, x_{2}=-b 1, x_{1}=-b 2$ \\
\hline
\end{tabular}

Note that notation " $\vee$ "refers to symbol of OR, while $\cup$ refers to a symbol for combination. 
As the result in Table 5 is observed, column 1 shows an input vector that is composed of every possible combination of input edges. Each input vector results in different parametric determinant equations where its additional must-exist edge is described in column 2. Column 3 subsequently derives a singularity point that may cause the $\operatorname{Det}\left(\left[\Delta_{1}\right]\right)=0$.

Based on the result described in Table 4 and 5, a few remarks can be made:

1. If the input to an SBTS system is represented by input edge(s) that connect(s) to state vertex 1 , then, in order to satisfy the Lie algebra rank condition, an additional edge that connects to state vertex 2 is required.

2. Vice versa, if the input to an SBTS system is represented by input edges that connect to state vertex 2 , then, in order to satisfy the Lie algebra rank condition, an additional edge that connects to state vertex 1 is required.

3. If these "input" and "must-exist" edges are composed in a sequence with input edge(s) as the starting point, it forms a path that connects input edges to every state of the given SBTS. A state digraph that contains this type of path is defined by Definition 5.4.2 as an input connectable system.

4. Singularity, that is, when the $\operatorname{Det}\left(\left[\Delta_{1}\right]\right)=0$, results in an unsatisfactory Lie algebra rank condition as its rank is less than $n$. Given the non-zero parameter's value, singularity happens only when the states ( $x$ 's) of the system reach singular points or equilibrium as described in column 3 of Table 5.

5. If there exist path with width exactly $\mathrm{n}$, a Lie algebra rank condition of full rank is most likely guaranteed. 
To verify this finding, consider an admissible third order thermal dynamic bilinear system which has been modified to have its digraph notations given by Equation 5.9:

$[\Sigma]: \dot{x}=\left[\begin{array}{ccc}E 0_{11} & E 0_{21} & E 0_{31} \\ E 0_{12} & E 0_{22} & E 0_{32} \\ E 0_{13} & E 0_{23} & E 0_{33}\end{array}\right]\left[\begin{array}{l}x_{1} \\ x_{2} \\ x_{3}\end{array}\right]+\left[\begin{array}{ccc}0 & 0 & 0 \\ 0 & E 1_{22} & 0 \\ 0 & 0 & 0\end{array}\right]\left[\begin{array}{l}x_{1} \\ x_{2} \\ x_{3}\end{array}\right] u_{1}$

Following the same approach, concurrence is obtained and described by Table 6:

Table 6: Structural Controllability Result Summary

\begin{tabular}{|c|c|c|}
\hline $\begin{array}{c}\text { Input Vector } \\
\left(g_{1}\right) \text { Parameters } \\
\text { (Edge/s) }\end{array}$ & $\operatorname{Det}\left(\left[\Delta_{1}\right]\right)$ Parameteric Equation & $\begin{array}{l}\text { Parameter/Edges } \\
\text { that must Exist for } \\
\qquad \operatorname{Det}\left(\left[\Delta_{1}\right]\right) \neq \mathbf{0}\end{array}$ \\
\hline$E 1_{22} \& E 1_{2} b 1_{2}$ & $\left(-E 0_{21} E 0_{21} E 0_{13}\right) E 1_{22} x_{2}\left(E 1_{22} x_{2}+B 1_{2}\right)^{2}$ & $\left\{E 1_{22}, E 0_{21}, E 0_{13}\right\}$ \\
\hline$E 1_{22} \& E 1_{2} b 1_{2}$ & $\left(-E 0_{21} E 0_{23} E 0_{33}+E 0_{21} E 0_{23} E 0_{11}\right) E 1_{22} x_{2}\left(E 1_{22} x_{2}+B 1_{2}\right)^{2}$ & $\left\{E 1_{22}, E 0_{21}, E 0_{23}\right\}$ \\
\hline$E 1_{22} \& E 1_{2} b 1_{2}$ & $\left(E 0_{23} E 0_{23} E 0_{31}\right) E 1_{22} x_{2}\left(E 1_{22} x_{2}+B 1_{2}\right)^{2}$ & $\left\{E 1_{22}, E 0_{23}, E 0_{31}\right\}$ \\
\hline$E 1_{22} \& E 1_{2} b 1_{2}$ & $\left(\begin{array}{l}-E 0_{21} E 0_{21} E 0_{13}-E 0_{21} E 0_{23} E 0_{33}+\ldots \\
E 0_{21} E 0_{23} E 0_{11}+E 0_{23} E 0_{23} E 0_{31}\end{array}\right) E 1_{22} x_{2}\left(E 1_{22} x_{2}+B 1_{2}\right)^{2}$ & $\left\{E 1_{22},\left(\begin{array}{c}E 0_{21}, E 0_{13} \ldots \\
\cup E 0_{21}, E 0_{23} \\
\cup E 0_{23}, E 0_{31}\end{array}\right)\right\}$ \\
\hline
\end{tabular}

This completes the proof of Lemma 5.4.1.

Based on the above discussion, Proposition 5.4.1 can now be proven for characterizing the structural controllability of an SBTS system using a graphical criterion.

\section{Proposition 5.4.1:}

An SBTS $[\Sigma]$ is said to be locally structurally controllable if and only of its state diagraph $G_{S}=[\Sigma]$ is input connectable. 


\section{Proof of Proposition 5.4.1:}

\section{Necessary}

Assume that the condition of Proposition 5.4.1 is not satisfied, such that its state

digraph $G_{S}=[\Sigma]$ is not input connectable according to Definition 5.4.2. Hence, based in Lemma 5.4.1, the given SBTS $[\Sigma]$ is lacking one or two things:

1. The given SBTS $[\Sigma]$ may not have input vectors even a single input edge cannot be derived. This implies inability to produce a controllability distribution $\Delta_{C}$, OR

2. There is at least one missing must-exist-edge which results in an unsatisfactory Lie algebra rank condition for controllability.

\section{Sufficiency}

Assume that the condition of Proposition 5.4.1 is satisfied, such that its state digraph $G_{S}=[\Sigma]$ is input connectable according to definition 5.4.2. Then the given SBTS is able to produce a structured controllability distribution $\left[\Delta_{C}\right]$ that satisfies the Lie algebra structural rank condition for structural controllability.

\section{Non-Controllability}

If a non-controllability aspect of an SBTS using this criterion is considered, it can be explained as follows: The proof of Lemma 5.4.1 shows that the singularity point of SBTS can cause an unsatisfactory result of the Lie algebra rank condition as the rank of the obtained controllability distribution is not a full rank. It was shown in Table 5 that singularity points include all states associated with input edges that are identical. As the 
state in SBTS represents temperature, the identical state value implies an equal temperature between the states, or, simply put, that the STBS is at its equilibrium state. Thus, no energy exchange will occur even though system input is varied. Therefore, this state makes the system un-controllable. The main advantage of Proposition 5.4.1 is its computational aspect which is very well suited to large-scale systems. The graphical criterion has an intuitive interpretation and is easy to check by hand. Furthermore, noncontrollability can also be identified easily by observation.

\subsection{Application}

In this section an illustration on the application of the proposed method is presented. The first part, an example of a 2 cell heat exchanger is utilized. This example is used to somewhat represent a large-scale structured nonlinear system. On the second part of this section, controllability of the BCHP system given in chapter four is investigated.

\subsubsection{Local Controllability of a nonlinear 2-cell Heat Exchanger}

An example of a cascade heat exchanger consists of a 2-cell heat exchanger in reverse flow as depicted by Figure 21. By definition, given that the system consists of 2 subsystems of heat exchanger that are interconnected, this particular system can be considered as a large-scale system. 


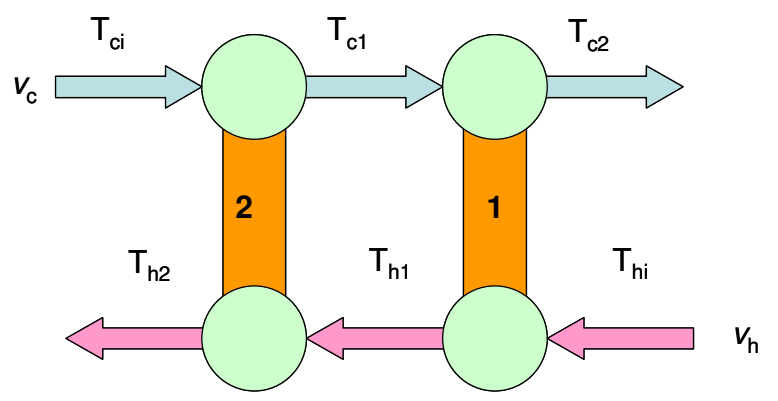

Figure 21: A Cascade Model of Heat Exchanger

Based on energy conservation, the following thermal dynamic equation is derived to model the heat exchanger where the following notation is applied:

$$
\begin{aligned}
& x_{1}=T_{c 2}, x_{2}=T_{h 1}, x_{3}=T_{c 1}, x_{4}=T_{h 2}, b_{1}=T_{h i} \text { and } b_{2}=T_{c i} \text {, where } u_{1}=v_{c} \text { and } u_{2}=v_{h} . \\
& \dot{x}_{1}=\frac{v_{c}}{V_{c 1}}\left(x_{3}-x_{1}\right)+k_{1}\left(x_{2}-x_{1}\right)=-k_{1} x_{1}+k_{1} x_{2}+\left(\frac{1}{V_{c 1}}\left(-x_{1}+x_{3}\right)\right) v_{c} \\
& \dot{x}_{2}=\frac{v_{h}}{V_{h 1}}\left(T_{h i}-x_{2}\right)+k_{2}\left(x_{1}-x_{2}\right)=k_{2} x_{1}-k_{2} x_{2}+\left(\frac{1}{V_{h 1}}\left(-x_{2}+T_{h i}\right)\right) v_{h} \\
& \dot{x}_{3}=\frac{v_{c}}{V_{c 2}}\left(T_{c i}-x_{3}\right)+k_{3}\left(x_{4}-x_{3}\right)=-k_{3} x_{3}+k_{3} x_{4}+\left(\frac{1}{V_{c 2}}\left(-x_{3}+T_{c i}\right)\right) v_{c} \\
& \dot{x}_{4}=\frac{v_{h}}{V_{h 2}}\left(x_{2}-x_{4}\right)+k_{4}\left(x_{3}-x_{4}\right)=k_{4} x_{3}-k_{4} x_{4}+\left(\frac{1}{V_{h 2}}\left(x_{2}-x_{4}\right)\right) v_{h} \\
& y_{j}(t)=\left[\begin{array}{l}
x_{1}(t) \\
x_{4}(t)
\end{array}\right]
\end{aligned}
$$

State equation 5.10 is transformed into a state space form as described by Equation 5.11: 


$$
\Sigma:\left\{\begin{aligned}
{\left[\begin{array}{l}
\dot{x}_{1} \\
\dot{x}_{2} \\
\dot{x}_{3} \\
\dot{x}_{4}
\end{array}\right]=} & {\left[\begin{array}{cccc}
-k_{1} & k_{1} & 0 & 0 \\
k_{2} & -k_{2} & 0 & 0 \\
0 & 0 & -k_{3} & k_{3} \\
0 & 0 & k_{4} & -k_{4}
\end{array}\right]+\left(\left[\begin{array}{cccc}
-\frac{1}{V_{c 1}} & 0 & \frac{1}{V_{c 1}} & 0 \\
0 & 0 & 0 & 0 \\
0 & 0 & -\frac{1}{V_{c 2}} & 0 \\
0 & 0 & 0 & 0
\end{array}\right]\left[\begin{array}{c}
x_{1} \\
x_{2} \\
x_{3} \\
x_{4}
\end{array}\right]+\left[\begin{array}{c}
0 \\
0 \\
\frac{1}{V_{c 2}} T_{c 1} \\
0
\end{array}\right]\right) v_{c}+\ldots } \\
& \ldots\left(\left[\begin{array}{cccc}
0 & 0 & 0 & 0 \\
0 & -\frac{1}{V_{h 1}} & 0 & 0 \\
0 & 0 & 0 & 0 \\
0 & \frac{1}{V_{h 2}} & 0 & -\frac{1}{V_{h 2}}
\end{array}\right]\left[\begin{array}{l}
x_{1} \\
x_{2} \\
x_{3} \\
x_{4}
\end{array}\right]+\left[\begin{array}{c}
0 \\
\frac{1}{V_{h 1}} T_{h 1} \\
0 \\
0
\end{array}\right]\right)
\end{aligned}\right.
$$

Subsequently, the structured model of Equation 5.11 based on a signed structured method given by Definition 2.4.2, is obtained and described by Equation 5.12. Hence, another transformation into a digraph is depicted in Figure 5.2.

$$
[\Sigma]:\left\{\begin{aligned}
{\left[\begin{array}{c}
\dot{x}_{1} \\
\dot{x}_{2} \\
\dot{x}_{3} \\
\dot{x}_{4}
\end{array}\right]=} & {\left[\begin{array}{cccc}
-E 0_{1,1} & E 0_{1,1} & 0 & 0 \\
E 0_{1,2} & -E 0_{22} & 0 & 0 \\
0 & 0 & -E 0_{3,3} & E 0_{4,3} \\
0 & 0 & E 0_{3,4} & -E 0_{4,4}
\end{array}\right]+\ldots } \\
& \cdots\left(\left[\begin{array}{cccc}
-E 1_{1,1} & 0 & E 1_{3,1} & 0 \\
0 & 0 & 0 & 0 \\
0 & 0 & -E 1_{33} & 0 \\
0 & 0 & 0 & 0
\end{array}\right]\left[\begin{array}{c}
x_{1} \\
x_{2} \\
x_{3} \\
x_{4}
\end{array}\right]+\left[\begin{array}{c}
0 \\
0 \\
E 1_{33} B 1_{3} \\
0
\end{array}\right]\right) v_{c}+\ldots \\
& \ldots\left(\left[\begin{array}{cccc}
0 & 0 & 0 & 0 \\
0 & -E 2_{2,2} & 0 & 0 \\
0 & 0 & 0 & 0 \\
0 & E 2_{2,4} & 0 & -E 2_{4,4}
\end{array}\right]\left[\begin{array}{c}
x_{1} \\
x_{2} \\
x_{3} \\
x_{4}
\end{array}\right]+\left[\begin{array}{c}
0 \\
E 2_{2,2} B 2_{2} \\
0 \\
0
\end{array}\right]\right) v_{h}
\end{aligned}\right.
$$

Structural controllability using the graph-theoretic approach is first performed in order to investigate the controllability of the system. The result then is confirmed by the analytical structural controllability method and the proposed method of graph-theoretic approach can be verified. Figure 22 depicts the overall digraph of the nonlinear 2-cell heat exchanger. Subsequently, the state diagraph is constructed and depicted on Figure 23. 


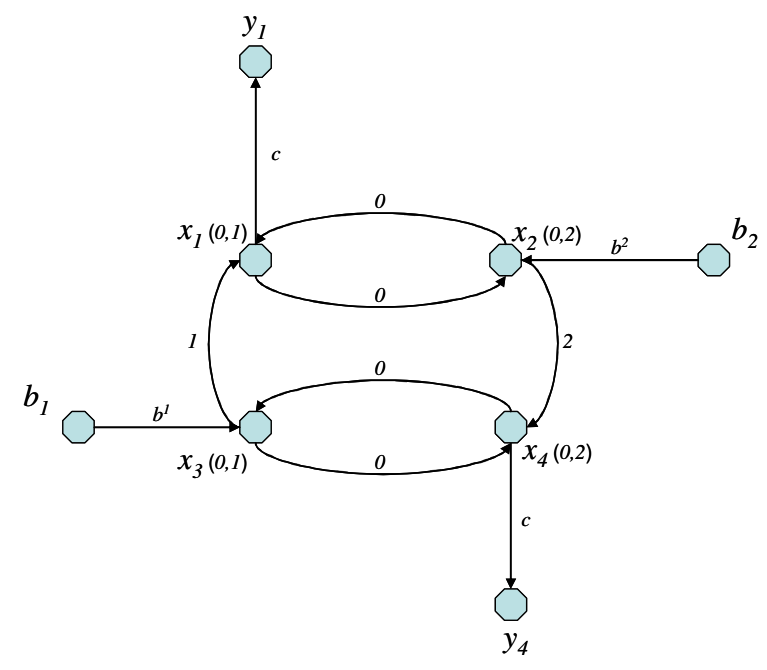

Figure 22: Digraph of 2-Cell Heat Exchanger

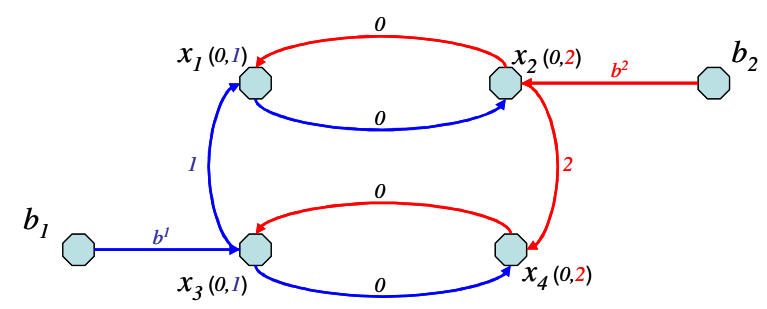

Figure 23: State Digraph of Nonlinear 2-Cell Heat Exchanger

Observing the state digraph of the nonlinear 2-cell heat exchanger depicted on Figure 23, it is clearly showed that the system is input connectable according to Definition 5.4.2. Hence, according to Proposition 5.4.1, the system is locally structurally controllable. This result is then verified against the analytical method of Theorem 5.3.1 for the structural controllability rank test condition using the structured controllability distribution $\left[\Delta_{C}\right]$. Applying Isidori's algorithm $[1995,1]$ for constructing controllability distribution, the following structured controllability distribution $\left[\Delta_{C}\right]$ is obtained. 


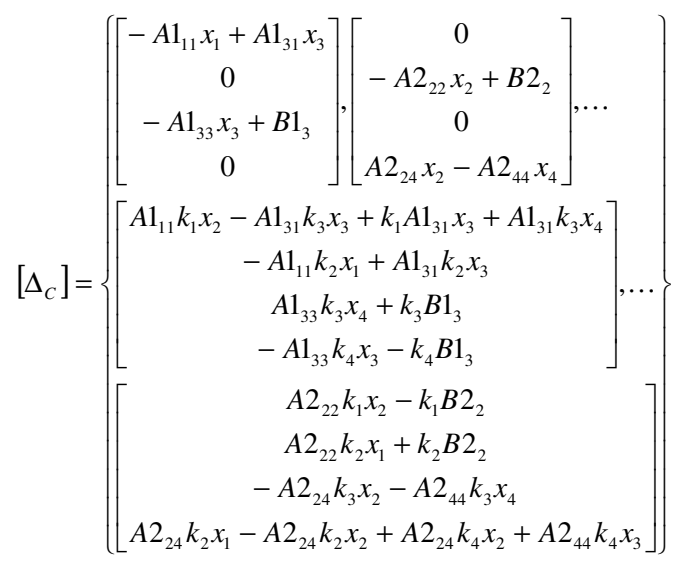

Equation 5.13 describes the controllability distribution $\left[\Delta_{C}\right]$ of nonlinear a 2-cell heat exchanger consisting of four vectors. The structural rank of $\left[\Delta_{C}\right]$ is four. Except at a singular point where the rank of the distribution decreases, that is, $x_{1}=x_{3}, x_{2}=T_{h 1}, x_{1}=$ $T_{c l}, x_{4}=x_{2}$. This singular point represents the equilibrium condition of the heat exchanger where the input and output temperature are equal. Therefore, any controllable input that is utilized will not control the output.

\subsubsection{Structural Controllability of BCHP system.}

Consider a BCHP system described in chapter four. The state digraph of the system is depicted again on Figure 24. In order to investigate the controllability of the BCHP system, a graph-theoretic approach is favorable due to the size of the system. According to Proposition 5.4.1, the BCHP state digraph is input connectable. Hence, the system is locally structurally controllable, except at singular points where the structural rank of controllability distribution of BCHP system is not a full rank. This condition is obtained due to the temperature equilibrium that is reached in the system, thus no heat transfer occurs regardless of whether or not the variable control input is varied. 


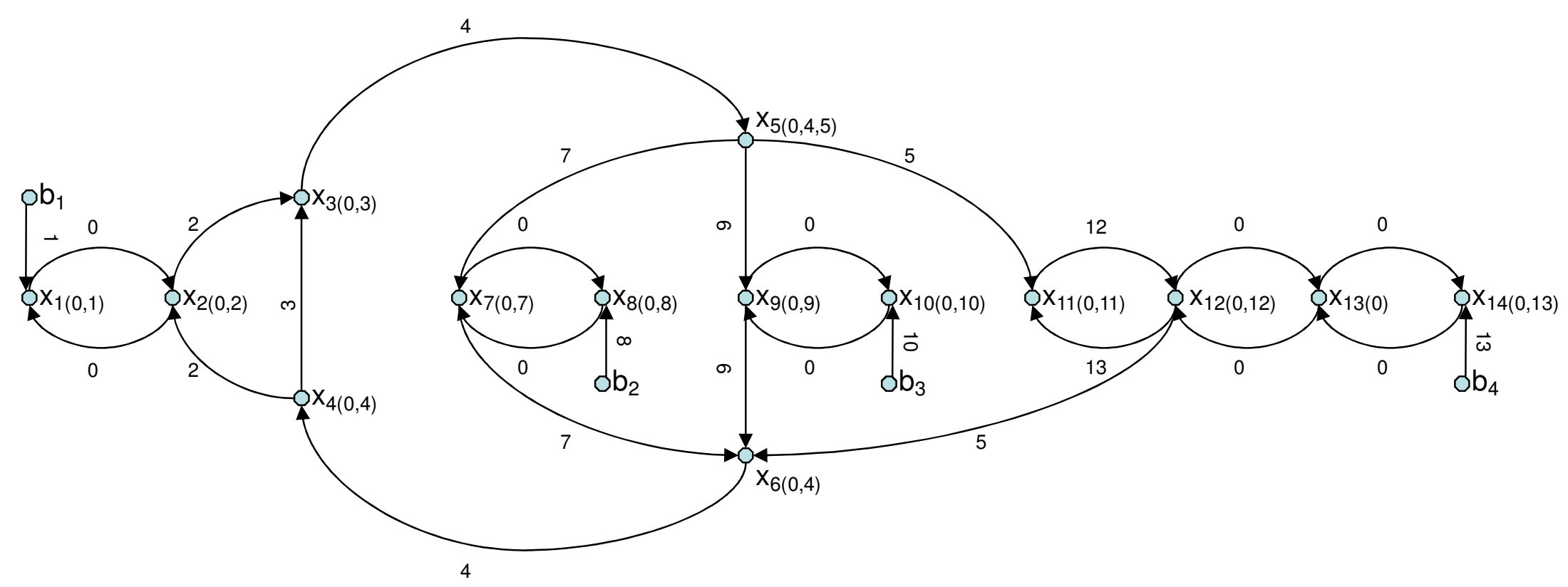

Figure 24: Input Connectable BCHP State Digraph 


\section{Chapter Six}

\section{OBSERVABILITY \\ OF STRUCTURED NONLINEAR THERMAL DYNAMIC SYSTEM VIA CONNECTABILITY APPROACH}

This chapter presents a new approach to observability of structured nonlinear systems using a graph-theoretic approach. On the basis of a digraph representation, the necessary and sufficient conditions for the observability of structured non-linear systems are expressed in graphic terms. These conditions have an intuitive interpretation and are easy to check by hand for small systems and by means of well-known combinatorial techniques for large-scale systems. The results presented here then serve as the analytic foundation for observability analysis for the research systems presented in the previous chapter.

\subsection{Observability of Non-Linear Thermal Dynamic System}

In this thesis, bilinear thermal dynamic system (BTS) as described by Equation 5.2 is considered: 


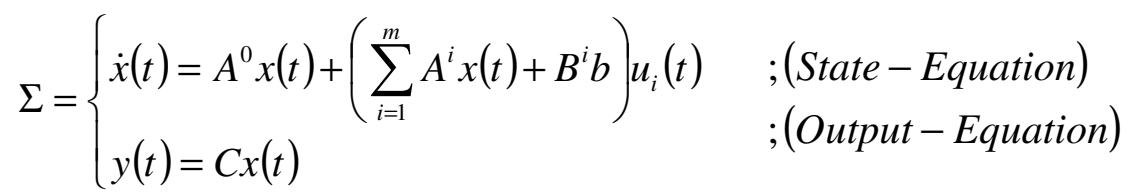

where $x(t)=\left(x_{1}(t), \ldots, x_{n}(t)\right)^{T} \in M \subset \Re^{n}, \quad u(t)=\left(u_{1}(t), \ldots, u_{m}(t)\right)^{T} \in \Omega \subset \Re^{m}$, $y(t)=\left(y_{1}(t), \ldots, y_{p}(t)\right)^{T} \in \Re^{p}$ are, the state, the input and the output vectors, respectively;

and $A^{0} \in \mathfrak{R}^{n \times n}$, for $i=1, \ldots, m, A^{i} \in \mathfrak{R}^{n \times n}, \quad B^{i} b \in \mathfrak{R}^{n \times 1}$, and $C \in \mathfrak{R}^{p \times n}$ are state space matrices form of $C^{\infty}$ function vector fields on $M$ representing the heat transfer mechanisms.

The problem statement of observability in the nonlinear system focuses on finding the condition where the initial state $x^{0}$ can be distinguished given the output measurement. This was previously described by Definition 2.3.4.

Definition 2.3.4 ( $U$-indistinguishable):

Given a subset $U \subseteq M$, and $x^{0}, x^{1} \in U$, it is said that $x^{0}$ is $U$-indistinguishablefrom $x^{1}\left(\right.$ denoted $\left.x^{1} I_{U} x^{0}\right)$ if for every control $\left(u(t),\left[t^{0}, t^{1}\right)\right.$ whose trajectories $\left(x^{0}(t),\left[t^{0}, t^{1}\right]\right)$ and $\left(x^{1}(t),\left[t^{0}, t^{1}\right)\right.$ from $x^{0}$ and $x^{1}$ both lie in $U$, fails to distinguish between $x^{0}$ and $x^{1}$, i.e., if $x^{0}(t) \in U$ and $x^{1}(t) \in U$ for $t \in\left[t^{0}, t^{1}\right]$, then $\left.\left.\Sigma_{x^{0}}\left(u(t), \mid t^{0}, t^{1}\right]\right)=\Sigma_{x^{1}}\left(u(t), \mid t^{0}, t^{1}\right]\right)$. Denote $I\left(x^{0}\right)=\left\{x^{1} \in M: x^{1} I x^{0}\right\}$ the set of points indistinguishable from $x^{0}$.

In the case of this research, local state observability is considered.

\section{Definition 2.3.6 (Local State Observability):}

The system $\Sigma$ is said to be locally observable at $x^{0}$ if for every neighborhood $U$ of $x^{0}$, $I_{U}\left(x^{0}\right)=\left\{x^{0}\right\} ; \Sigma$ is locally observable if this is true for every $x \in M$. 
Furthermore, in order to investigate the local observability of a nonlinear system, observability rank test condition is performed on the derivative of observation space $\Omega$. This was discussed earlier in chapter two.

\section{Theorem 2.3.2 (Observability Rank Test):}

$\operatorname{SBTS} \Sigma$ satisfies the observability rank condition at $x^{0}$ if in a neighborhood of $x^{0}$, $\operatorname{dim}\left[d \Omega_{O}\right]=n$. If this holds for all $x^{0} \in M$, then $\Sigma_{\text {satisfies }}$ the observability rank condition. Thus, if $\Sigma$ satisfies the observability rank condition at $x^{0} \in M$, then $\Sigma$ has the local distinguishability property at $x^{0}$.

Therefore, the first step toward the analysis of local observability of nonlinear systems is to construct the observability co-distribution that is based on Observation space $O$ using Lie derivative on output function and vector field. Isidori [1995, 1] proposes an algorithm for constructing the observability co-distributions $d O$ as follows:

\section{Algorithm for Constructing the Observability Co-Distribution}

4. Starting Point: $\Omega_{0}=\operatorname{span}\left\{d h_{1}, \ldots, d h_{p}\right\}$

5. Development of the controllability Distribution: $\Omega_{k}=\Omega_{k-1}+\sum_{i=0}^{m} L_{g_{i}} \Omega_{k-1}$

6. Stopping criterion: if there exist an integer $k^{*}$ such that $\Omega_{k^{*}}=\Omega_{k^{*-1}}$, then

$$
\Omega_{0}=\Omega_{k^{*}}=\left\langle g_{0}, \ldots, g_{m} \mid \Omega_{0}\right\rangle
$$

where $g_{0}=A^{0} x$ and $g_{i}=\left(A^{i} x+B^{i} b\right)$ 


\subsection{Observability of Structured Non-Linear Thermal Dynamic System}

When the concern in the observability analysis is in the form of structured matrices, structural observability is applied to ensure the given structured system is observable. Hence, the following definition is applied.

\section{Definition 6.2.1 (Structural Observability):}

A class of nonlinear systems given by its structure matrix pair $\left[A^{0}, A^{i}, C\right]$ for $i=1, \ldots, m$ is said to be structurally observable (for short, s-observable) if there exist at least one admissible realization $\left(A^{0}, A^{i}, C\right) \in\left\lfloor A^{0}, A^{i}, C\right\rfloor$ being observable in the usual numerical sense.

Furthermore in order to determine if the structured bilinear thermal dynamic system is structurally observable at $x^{0}$, based on theorem 2.3.2, the necessary and sufficient conditions that must be satisfied transformed into the following theorem.

\section{Theorem 6.2.1:}

Structured nonlinear thermal dynamic systems $[\Sigma]$ characterized by structure matrix pair $\left\lfloor A^{0}, A^{i}, C\right\rfloor$ for $i=1, \ldots, m$ is structurally locally observable if, for almost all the realization of $(\Sigma) \in[\Sigma]$, there holds observability co-distribution $\left(\Omega_{O}\right) \in\left[\Omega_{O}\right]$ of structural $\operatorname{dimension} n$.

Note that structural dimension here is equivalent to structural rank of Definition 2.4.5. 


\subsection{Observability of Structured Non-Linear Thermal Dynamic System via Connectability Approach}

This section focuses on providing the graphic conditions equivalent to the one of Theorem 6.2.1 on structural observability rank condition for structured nonlinear systems. Since the focus of the research is placed upon bilinear thermal dynamic systems (BTS), the first part of this section is devoted to some definition of a digraph utilized as the tools for analyzing structural observability. The second part discusses the proposed graphical criterion that characterized the structural observability of structured bilinear thermal dynamic systems (SBTS).

\subsubsection{Digraph Definition for Structured Thermal Dynamic System}

Given a structured bilinear thermal dynamic system $[\Sigma]$, some definitions based on digraph theory described in chapter three is devised:

\section{Definition 6.3.1 (Output Digraph):}

Output digraph $G_{O}([\Sigma])$ is a digraph $G=(V, E)_{\text {of a }}$ a structured bilinear system $[\Sigma]$ represented by its state space model of equation 5.2, and is characterized by the following:

a. The vertex set $V$ is given by $Y \cup X$ with $Y=\left\{y_{1}, \ldots, y_{p}\right\}$ the set of output vertices, and $X=\left\{x_{1}, \ldots, x_{n}\right\}$ the set of state vertices. 
b. The edge set $E$ is described by $E_{A^{l}} \cup E_{C}$ with $E_{A^{l}}=\left\{\left(x_{j}, x_{i}\right)\left[\left[A^{l}\right]_{i, j} \neq 0\right\}\right.$ $l=0,1, \ldots, m$, and $E_{C}=\left\{\left(u_{j}, x_{i}\right)\left[[C]_{i, j} \neq 0\right\}\right.$.

For $l=0,1, \ldots, m$ an index $l$ is assigned to each edge $e \in E_{A^{l}} \cup E_{C}$. Note that several indexes may be given to an edge $e$ if it belongs to several subsets $E_{A^{l}}-e d g e s$. For $l=1, \ldots, m$, this index correspond to system input $u_{l}$.

\section{Definition 6.3.2 (Output Connectable):}

Output digraph $G_{O}([\Sigma])$ is output connectable if paths exist from every state vertex to each of the output vertex consisting at least $m$ distinct input edges.

\subsubsection{Main Results}

Observability is the dual concept of controllability, thus any statement about controllability has its direct counterpart concerning observability. Therefore, an obvious precondition of observability is that the system outputs are able to influence all state variables. Said in graph-theoretic terms for bilinear system, paths must exist from each state vertex to each output vertice. Therefore, the following proposition is suggested in this research:

\section{Proposition 6.3.1:}

A structured bilinear thermal dynamic system (SBLTD) $[\Sigma]$ is structurally observable if and only if in its associated output diagraph $G_{O}([\Sigma])$ is output connectable.

In order to prove Proposition 6.3.1, it is important to show that ouput-connectable systems possess the ability to satisfy the necessary and sufficient conditions for a bilinear 
system to be observable in the standard numerical sense of Lie algebra rank condition as described in Theorem 6.2.1. Thus, the following lemma is formulated:

\section{Lemma 6.3.1:}

If an $n$-order SBTS $[\Sigma]$ characterized by structure matrix pair $\left[A_{l=0}^{m}, C\right] l=0,1, \ldots, m$ is output connectable, then there holds a structured matrix formed by observability codistribution $\left[\Omega_{O}\right]$ of $[\Sigma]$ with rank $n$ for almost all the realization of $(\Sigma) \in[\Sigma]$.

\section{Proof of Lemma 6.3.1}

In order to verify Lemma 6.3.1, $n$-order SBTS is studied gradually to find the one that would guarantee resulting in satisfying the Lie algebra rank condition. It will be shown that in order to obtain a structured observability distribution $\Omega_{O}$ of $[\Sigma]$ with rank $n$ for almost all the realization of $\Sigma$, a certain connectability property, that is, output connectability must exist.

First, consider an admissible second order SBTS $(n=2)$ with single input $(m=1)$ and observable output, that is, state $x_{1}$, as described by the following state Equation:

$\Sigma:\left\{\begin{array}{l}\dot{x}=\left[\begin{array}{cc}-k s_{1} & k s_{1} \\ k s_{2} & -k s_{2}\end{array}\right]\left[\begin{array}{l}x_{1} \\ x_{2}\end{array}\right]+\left[\begin{array}{cc}-k 1_{1} & k 1_{1} \\ k 1_{2} & -k 1_{2}\end{array}\right]\left[\begin{array}{l}x_{1} \\ x_{2}\end{array}\right] u_{1}+\left[\begin{array}{l}b 1_{1} \\ b 1_{2}\end{array}\right] u_{1} \\ y=\left[\begin{array}{ll}1 & 0\end{array}\right]\left[\begin{array}{l}x_{1} \\ x_{2}\end{array}\right]\end{array}\right.$

To create a relationship that is proposed by Lemma 6.3 .1 , sign structure model $[\Sigma]$ is utilized. Thus Equation 6.1 now becomes the following state equation: 


$$
[\Sigma]:\left\{\begin{array}{l}
\dot{x}=\left[\begin{array}{cc}
-E 0_{1,1} & E 0_{2,1} \\
E 0_{1,2} & -E 0_{2,2}
\end{array}\right]\left[\begin{array}{l}
x_{1} \\
x_{2}
\end{array}\right]+\left[\begin{array}{cc}
-E 1_{1,1} & E 1_{2,1} \\
E 1_{1,2} & -E 1_{2,2}
\end{array}\right]\left[\begin{array}{l}
x_{1} \\
x_{2}
\end{array}\right] u_{1}+\left[\begin{array}{l}
b 1_{1} \\
b 1_{2}
\end{array}\right] u_{1} \\
y=\left[\begin{array}{ll}
E Y_{1,1} & 0
\end{array}\right]\left[\begin{array}{l}
x_{1} \\
x_{2}
\end{array}\right]
\end{array}\right.
$$

Given an SBTS described by Equation 6.2, parameter $E k_{i, j}$ represents both the structured entry of $A_{k}$ matrix of $i$-th column and $j$-th row but also the edge that connects state vertex $i$ to state vertex $j$ associated with matrix $A_{k}$. Furthermore $b k_{j}$ refers to the entry of $k$-th input of structured vector $B_{k}$ of row $j$-th, which also represents the edge from vertex $b k_{j}$ to state vertex $j$. On the output equation side $E Y_{i, j}$ represents both the structured entry of $C$ matrix of $i$-th column and $j$-th row and also the edge that connects state vertex $i$ to state vertex $j$ associated with matrix $C$. For the completeness, based on this substitution of parameter notation the following equality is:

5. $E 0_{1,1}=E 0_{2,1}=k s_{1}$

6. $E O_{1,2}=E O_{2,2}=k s_{2}$

7. $E 1_{1,1}=E 1_{2,1}=E 1_{1}=k 1_{1}$

8. $E 1_{1,1}=E 1_{2,1}=E 1_{2}=k 1_{2}$

9. $E Y_{1}=1$

Using this SBTS matrix equation, Isidori's algorithm [1995, 1] for observability co-distribution $\left[\Omega_{o}\right]$ of $[\Sigma]$ is applied and the following structured matrix consisting of three vectors is obtained:

$\Omega_{1}=\operatorname{span}\left\{[1 \quad 0],\left[E 0_{11} \quad E 0_{21}\right],\left[\begin{array}{ll}E 1_{11} & E 1_{21}\end{array}\right]\right\}$ 
To check the Lie algebra rank condition of the structured matrix $\Omega_{1}$, its determinant (Det $\left[\Omega_{1}\right]$ ) is subsequently evaluated. Equation 6.4 described two different possible structured parametric equations of $\operatorname{Det}\left[\Omega_{1}\right]$ :

$\Omega_{1}=\left[(1)\left(E 0_{21}\right)\right]$

$\Omega_{1}=\left[(1)\left(E 1_{21}\right)\right]$

In order to satisfy Lie algebra rank condition, a non-zero Det $\left[\Omega_{1}\right]$ is required. Using the structured parametric equation described by Equation x.4, it is observed that non-zero parameter of $E O_{21}$ or $E 1_{21}$ would result in non-zero determinant if state $x_{1}$ is observable. Vice versa, if state $x_{2}$ is the observable state as described by Equation 6.5 , using the same approach, the following structured parametric equation of $\operatorname{Det}\left[\Omega_{1}\right]$ is obtained:

$[\Sigma]:\left\{\begin{array}{l}\dot{x}=\left[\begin{array}{cc}-E 0_{1,1} & E 0_{2,1} \\ E 0_{1,2} & -E 0_{2,2}\end{array}\right]\left[\begin{array}{l}x_{1} \\ x_{2}\end{array}\right]+\left[\begin{array}{cc}-E 1_{1,1} & E 1_{2,1} \\ E 1_{1,2} & -E 1_{2,2}\end{array}\right]\left[\begin{array}{l}x_{1} \\ x_{2}\end{array}\right] u_{1}+\left[\begin{array}{l}b 1_{1} \\ b 1_{2}\end{array}\right] u_{1} \\ y=\left[\begin{array}{ll}0 & 0 E Y_{2,1}\end{array}\left[\begin{array}{l}x_{1} \\ x_{2}\end{array}\right]\right.\end{array}\right.$

$\Omega_{1}=\left[(1)\left(E 0_{12}\right)\right]$

$\Omega_{1}=\left[(1)\left(E 1_{12}\right)\right]$

Thus, non-zero parameter of $E 0_{12}$ or $E 1_{12}$ would result in non-zero determinant if state $x_{2}$ is observable. Table 7 summarizes the result of this finding: 
Table 7: Edge/s that maintain SBTS observability

\begin{tabular}{|c|c|c|}
\hline Observability State & $\begin{array}{c}\text { Output Equation } \\
\text { Parameter }\end{array}$ & $\begin{array}{c}\text { Edge that must Exist for } \\
\operatorname{Det}\left[\Omega_{1}\right] \neq \mathbf{0}\end{array}$ \\
\hline$x_{1}$ & $E Y_{1}$ & $E 0_{21}$ or $E 1_{21}$ \\
\hline$x_{2}$ & $E Y_{2}$ & $E 0_{12}$ or $E 1_{12}$ \\
\hline
\end{tabular}

It is observed from Table 7 that at least one non-zero parameter must exist in addition to the output parameter. Given that each parameter also represents an edge that connects initial vertex to terminal vertex, thus, based on this observation, it implies that given an of output edge $E Y_{i}$ which represents the observable state $x_{i}$, an additional edge with terminal vertex state $x_{i}$ is required.

Based on the result described in Table 8 a few remarks can be made:

6. If the output to an SBTS system is represented by an output edge that connects through state vertex 1 , then, in order to satisfy Lie algebra rank condition, an additional edge that connects from state vertex 2 to state vertex 1 is required.

7. Vice versa, if the output to an STBLD system is represented by an output edge that connects through state vertex 2 , then, in order to satisfy Lie algebra rank condition, an additional edge that connects from state vertex 1 to state vertex 2 is required.

8. As these "output" and "must-exist" edges are composed in a sequence with the mustexist edge as the starting point, it forms a path that connects output edges from every state of the given SBTS system. An output digraph that contains this type of path in previous sections is defined as an output connectable system.

9. If path/s exist/s with width exactly $n$, Lie algebra rank condition of full rank is most likely guaranteed 
To verify this finding, let's consider an admissible third order TBLD which has been modified to have its digraph notations given by Equation 6.7:

$[\Sigma]:\left\{\begin{array}{l}\dot{x}=\left[\begin{array}{lll}E 0_{11} & E 0_{21} & E 0_{31} \\ E 0_{12} & E 0_{22} & E 0_{32} \\ E 0_{13} & E 0_{23} & E 0_{33}\end{array}\right]\left[\begin{array}{l}x_{1} \\ x_{2} \\ x_{3}\end{array}\right]+\left[\begin{array}{ccc}0 & 0 & 0 \\ 0 & E 1_{22} & 0 \\ 0 & 0 & 0\end{array}\right]\left[\begin{array}{l}x_{1} \\ x_{2} \\ x_{3}\end{array}\right] u_{1} \\ y=\left[\begin{array}{lll}E Y_{11} & 0 & 0\end{array}\right]\left[\begin{array}{l}x_{1} \\ x_{2} \\ x_{3}\end{array}\right]\end{array}\right.$

Following the same approach, the must-exist-edges which verify the earlier remarks are obtained:

Table 8: Structural Observability Results

\begin{tabular}{|c|c|c|}
\hline \multirow{2}{*}{ Observability State } & $\begin{array}{c}\text { Output Equation } \\
\text { Parameter }\end{array}$ & $\begin{array}{c}\text { Edge that must Exist for } \\
\text { Det }\left[\Omega_{1}\right] \neq \mathbf{0}\end{array}$ \\
\hline$x_{1}$ & $E Y_{1}$ & $E 0_{21} \cup E 0_{31}$ \\
\hline$x_{1}$ & $E Y_{1}$ & $E 0_{21} \cup E 0_{32}$ \\
\hline$x_{1}$ & $E Y_{1}$ & $E 0_{31} \cup E 0_{23}$ \\
\hline
\end{tabular}

This completes the proof of Lemma 6.3.1.

Based on the above discussion, Proposition 6.3.1 for characterizing the structural observability of an STBLD system using a graphical criterion can now be proven.

\section{Proposition 6.3.1:}

An SBTS $[\Sigma]$ is said to be locally structurally observable if and only of its output diagraph is output connectable. 


\section{Proof of Proposition 6.3.1:}

Necessary

Assume that the condition of Proposition 6.3.1 is not satisfied, such that, its output

digraph is not output connectable. Then based on the proof of Lemma 3.2, then the given STBLD system is lacking one or two things:

1. The digraph of the given STBLD may not have output vectors that even a single output edge cannot be derived. This implies inability to even produce an observability distribution $\Omega_{O}$, OR

2. There is at least one missing must-exist-edge which results in unsatisfactory Lie algebra rank condition for observability.

\section{Sufficiency}

Assume that the condition of Proposition 6.3.1 is satisfied, such that, its output digraph is output connectable. Then the given SBTS system is able to produce an observability co-distribution $\Omega_{O}$ that satisfies the Lie algebra rank condition for structural observability.

\subsection{Application}

In this section an illustration on the application of the proposed method is presented. In the first part, an example of a 2 cell heat exchanger is utilized. This is used 
as an example of a large-scale structured nonlinear system. On the second part of this section, controllability of the BCHP system given in chapter four is investigated.

\subsubsection{Local Observability of a nonlinear 2-cell Heat Exchanger}

An example of a cascade heat exchanger consisting of a 2-cell heat exchanger reverse flow as depicted by Figure 25 is considered.

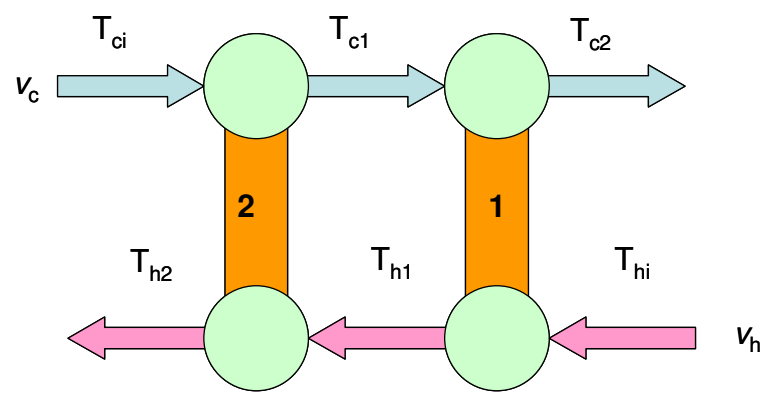

Figure 25: A Cascade Model of Heat Exchanger

In the case of the observability analysis, note that the measurable outpout of the system is $x_{1}=T_{c 2}$ and, $x_{4}=T_{h 2}$. Hence the same digraph as previously obtained is considered again here.

Structural observability using graph-theoretic approach is first performed in order to investigate the observability of the system. The result is then confirmed by the analytical structural observability method thus the proposed method of graph-theoretic approach can be verified. Figure 26 depicts the overall digraph of the nonlinear 2-cell heat exchanger. Subsequently, an output diagraph is constructed using Definition 6.3.2 and the result is depicted on Figure 27.

Observing the output digraph of the nonlinear 2-cell heat exchanger depicted on Figure 6.3, it clearly shows that the system is output connectable according to Definition 
6.4.2. Hence, according to Proposition 6.4.1 the system is locally structurally observable. This result is then verified against the analytical method of Theorem 6.2 .1 for structural observability rank test condition using the structured observability co-distribution $\left[\Omega_{o}\right]$. Applying Isidori's algorithm [1995, 1] for constructing observability co- distribution, the following structured obeservability distribution $\left[\Omega_{o}\right]$ is obtained.

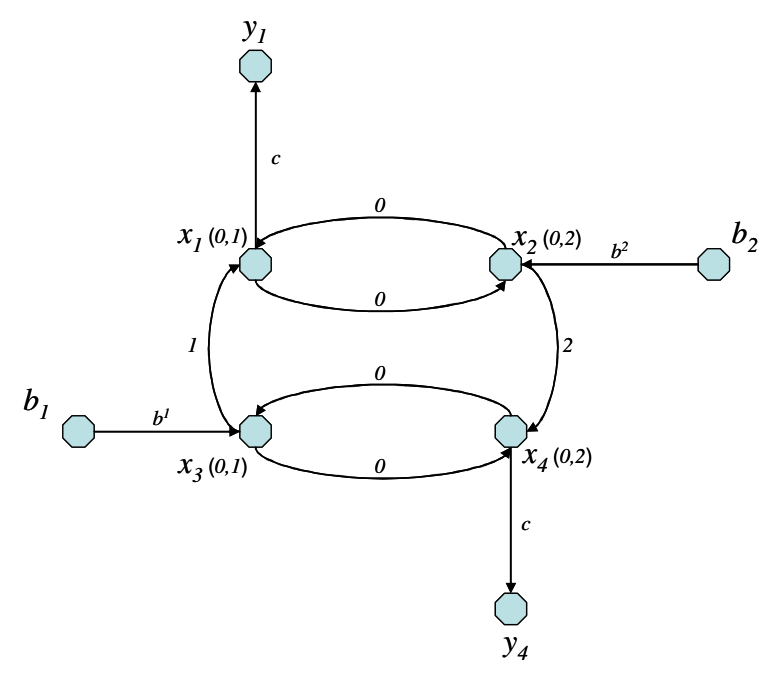

Figure 26: Digraph of 2-Cell Heat Exchanger

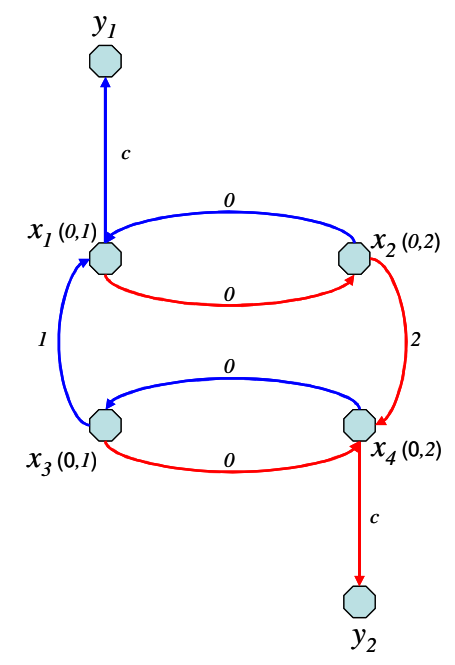

Figure 27: Output Digraph 


$$
\left[\Omega_{0}\right]=\left\{\begin{array}{l}
{\left[\begin{array}{cccc}
1 & 0 & 0 & 0 \\
0 & 0 & 0 & 1
\end{array}\right],\left[\begin{array}{cccc}
-E 0_{1,1} & E 0_{2,1} & 0 & 0 \\
0 & 0 & E 0_{3,4} & -k_{4}
\end{array}\right], \ldots} \\
\left.\left[\begin{array}{cccc}
-E 1_{1,1} & 0 & E 1_{3,1} & 0 \\
0 & 0 & 0 & 0
\end{array}\right],\left[\begin{array}{cccc}
0 & 0 & 0 & 0 \\
0 & E 2_{2,4} & 0 & -E 2_{4,4}
\end{array}\right]\right\}
\end{array}\right\}
$$

Equation 6.8 describes the structured observability co-distribution $\left[\Omega_{0}\right]$ of a nonlinear 2-cell heat exchanger consisting of six vectors. The structural rank of $\left[\Omega_{o}\right]$ is four. This shows that the proposed method of graph-theoretical approach is able to deduce the same conclusion as the analytical one. The main advantage of Proposition 6.3.1 is its computational aspect which is very well suited to large-scale systems. The graphical criterion has an intuitive interpretation and is easy to check by hand.

\subsubsection{Structural Observability of BCHP System}

The BCHP system described in chapter four is considered again. The actual set up of the BCHP system in the field is actually fully observable since there is a temperature sensor on every state of the system. For the purpose of this analysis, it is assumed that only a few measurements are available, that is:

Table 9: BCHP Measurable Output

\begin{tabular}{|l|c|}
\hline \multicolumn{1}{|c|}{ Definition } & $\begin{array}{c}\text { Large Scale } \\
\text { System State }\end{array}$ \\
\hline Microturbine-HX Air Outlet temperature & $x_{1}$ \\
\hline DWH-HX "A" - Water Outlet temperature & $x_{8}$ \\
\hline DWH-HX "B" - Water Outlet temperature & $x_{10}$ \\
\hline Building Space Air temperature & $x_{14}$ \\
\hline
\end{tabular}


This measured output can be seen from the BCHP diagraph depicted in Figure 6.4. Applying Proposition 6.4.1 on the BCHP output digraph, the structural observability is investigated. From observation of the output digraph, it is clearly shown that the diagraph is output connectable according to Definition 6.3.2. There are paths from each state vertex to every output vertex, which includes 12 distinct input edges. Therefore, the BCHP system is structurally locally observable according to proposition 6.4.1. This exercise is very useful in determining the number of sensors to be installed, as well as the selection of location. Therefore the number of sensors that need to be installed can be reduced while still maintaining the information needed for observation and control purposes. 


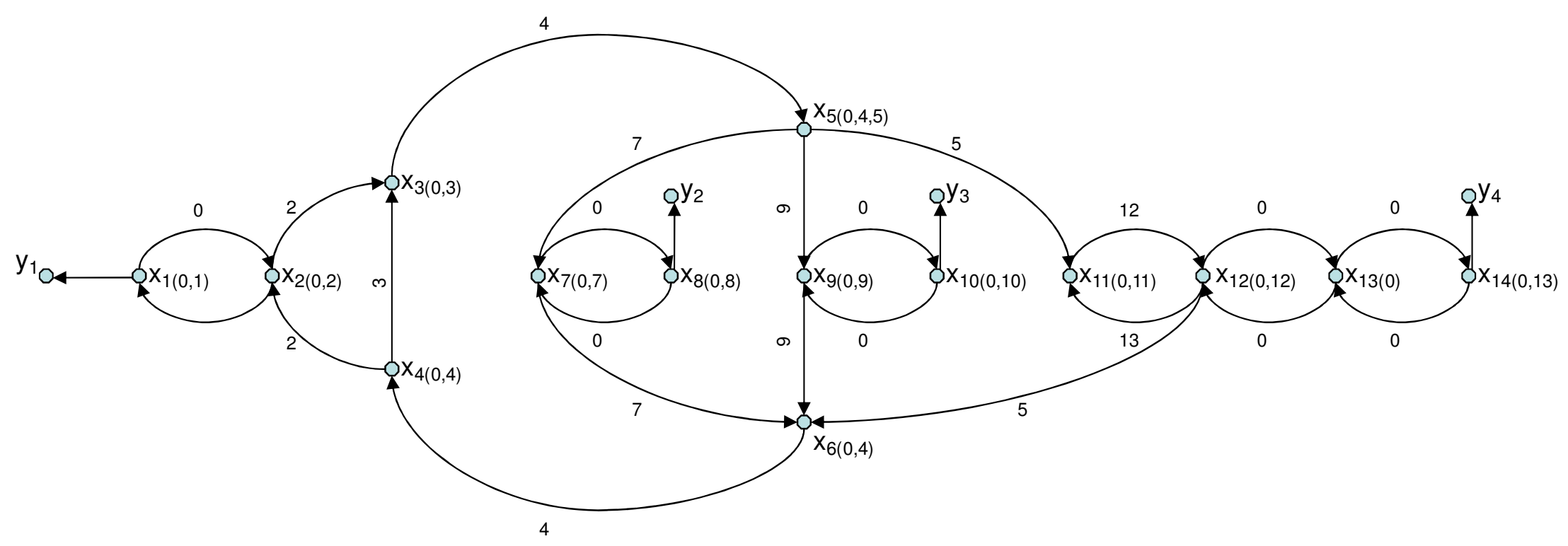

Figure 28: Output Digraph of BCHP system 


\section{Chapter Seven}

\section{CONCLUSION AND FUTURE WORK}

\subsection{Summary and Conclusions}

In summary, the primary objective for this research was to develop a methodology for determining the controllability and observability of a large-scale nonlinear thermal dynamic system. As an alternative to the often difficult and computationally intensive analytical method of analyzing the structural property of a large-scale nonlinear system, graph-theoretical approach is proposed in this research. Using a new graph representation of a special class of nonlinear system - bilinear system - a necessary and sufficient condition for structural controllability and observability are given and expressed in graphic terms. This method needs information that can be observed from its system digraph, and is easy to check which makes it well suited to analyze the large-scale system of thermal dynamic bilinear system. The contributions of the thesis are as follows:

\section{Digraph Model for a Bilinear System:}

- In many modeling problems, the exact knowledge of the state space matrices is sometimes unknown. To mitigate this deficiency, for a model with its state space system determined by the physical laws, some 
structured form can be defined. The study of such a model is called the structured modeling approach. This particular technique requires a low computational burden which allows one to deal with a large-scale system.

- Many results on structured systems are related to the graph theoretic approach. However, this approach is mainly dedicated to a linear system and many do not deal with nonlinear systems. In this research, a graph representation for a bilinear system was developed using directed-graph. Some definitions that explore the property of connectability for a bilinear system was derived here.

\section{Controllability and Observability of a Large-Scale Structured Bilinear Thermal Dynamic System via Connectability Approach}

- A new analysis tool to investigate the structural controllability and observability of a structured thermal dynamic bilinear system is proposed. The necessary and sufficient condition for structural controllability and observability which normally is determined using the analytical method of Lie algebra rank condition is now represented by graph representation. From a computational point of view, the proposed approach is particularly suited for a large-scale system since it is free from numerical difficulties. The proposed condition can be easily implemented because the method requires simple computations based on finding paths in digraphs. Furthemore, the use of graph-theoretic approach makes it easy to visualize the system structure. This may be very helpful for the optimization of 
actuator placement and sensor placement to achieve the controllability and observability of the system.

\subsection{Future Work}

This research work focused on a new practical approach to investigate the controllability and observability of a large-scale nonlinear thermal dynamic system, and there are several areas of possible future work. Based on the result presented in this thesis there are several areas which are especially relevant:

- Currently the technique to investigate the controllability and observability of a large scale nonlinear system is geared toward the structured system, such as a thermal dynamic system following thermodynamic principle. However, there are many other structured nonlinear systems following the engineering first principle that can be explored. Given that to a structured system a directed graph can naturally be associated, graph-theoretic approach still has a lot of potential to be utilized for structural properties analysis. 
Bibliography

Anguelova, Milena. 2004. Nonlinear Observability and Identifiability: General Theory and A Case Study of a Kinetic Model for S. cerevisiae. Licentiate of Engineering Thesis, Chalmers-Goteborg University.

Asamoah, F. and Jamshidi, Mohammad. 1986. Lyapunov Stability of Bilinear Large Scale Systems. Large Scale Systems 11: 69-79.

Balakrishnan, A. V. 1966. On the Controllability of Nonlinear Systems. Proceeding of National Academic of Science 55: 465-568

Bhandarkar, M. and Fahmy, M. 1972. Controllability of tandem-connected systems. IEEE Transactions on Automatic Control_17, no.1: 150- 151.

Bhat, Sanjay P. 2007. Controllability of Nonlinear Systems. Lecture, Short Course on Geometric Mechanics and Nonlinear Control. Department of Aerospace Engineering, Indian Institute of Technology, Mumbai, India. 
Bokor, József, Hangos, Katalin M., and Szederkényi, Gábor. 2004. Analysis and Control of Nonlinear Process Systems. London: Springer-Verlag.

Bokor, József, Hangos, Katalin M., and Szederkényi, Gábor. 2004. Analysis and Control of Nonlinear Process Systems. London: Springer-Verlag.

Bokor, József, Hangos, Katalin M., and Szederkényi, Gábor. 2004. Analysis and Control of Nonlinear Process Systems. London: Springer-Verlag.

Bornard, G. and Hammouri, H 2002. A Graph Approach to Uniform Observability of Linear Multi Output Systems. Proceedings of the $41^{\text {st }}$ IEEE Conference on Decision and Control: 701-706. Las Vegas, NV.

Boukhobza, T. and Hamelin F. 2007. Observability Analysis for Structured Bilinear System: A Graph-Theoretic Approach. Automatica 43: 1968-1974

Brasch, F.M. and Pearson, J.B. 1971. On the Controllability of Cascade Linear Systems. IEEE Trans Automatic Control 16, no.2 : 205-206.

Braun, James E., and Chaturvedi, Nitin. 2002. An Inverse Gray-Box Model for Transient Building Load Prediction. HVAC\&R Research 8, no. 1: 73-99. 
Brian Hopkins and Robin J. Wilson, "The Truth about Königsberg," The College Mathematics Journal, Vol. 35, No. 3, May 2004, pp. 198-207. http://curvebank.calstatela.edu/graphtheory/grthelink/grthelink.htm

Brittain, C.R., Otaduy, P. J., Perez, R. B., and Rovere, L. A. 1988. A New Approach to Hierarchical Decomposition of Large-Scale Systems. Proceedings of IEEE International Symposium on Intelligent Control: 108-112.

Brockett, R. W. 1972. Systems Theory on Group Manifolds and Co-Set Spaces. Journal of Society for Industrial and Applied Mathematics: Control 10: 265-284.

Brockett, R. W. 1975. On the Reachable Sets of Bilinear Systems. In Variable Structure Systems with Application to Economic and Biology, ed. Mohler, R. R. and Ruberti, A., 54-63. New York: Springer.

Cameron, Ian T. and Hangos, Katalin. 2001. Process Modeling and Model Analysis. Ed. Perkins, John and Stephanopulos, George. San Diego: Academic Press.

Caretheodory, C. 1967. Calculus of Variations and Partial Differential Equations of the First Order. San Francisco: Holden-Day.

Chellaboina, VijayShekhar, Haddad, Wassim M., and Nersesov, Sergey G. 2005. Thermodynamics: A Dynamical Systems Approach. Princeton: Princeton University Press 
Chen, Chi-Tsong and Desoer, C.A. 1970. Controllability and Observability of Composite Systems. IEEE Transactions on Automatic Control 15, no.2: 280-281.

Commault, Christian, Dion, Jean-Michael, and Hovelaque, V. 1997. A Geometric Approach for Structured Systems: Application to the Disturbance Decoupling Problem . Automatica 33: 403-409.

Commault, Christian, Dion, Jean-Michael, and Van der Woude, Jacob. 2003. Generic Properties and Control of Linear Structured Systems: A Survey. Automatica 39: 1125-1144.

Davison, E. J. 1977. Connectability and Structural Controllability of Composite System. Automatica 13: 109-123.

Davison, E. J. and Wang, S. H. 1973. Properties of Linear Time-Invariant Multivariable Systems subject to Arbitrary Output and State-Feedback. IEEE Transactions on Automatic Control 18: 24-32.

Davison, E. J. and Wang, S. H. 1975. New Results on the Controllability and Observability of General Composite Systems. IEEE Transactions on Automatic Control 20, no.1: 123-128. 
De Araujo, Humberto X., Mendes, Nathan, and Oliveira, Gustavo. 2001. Building Thermal Performance Analysis by Using MATLAB/SIMULINK. Paper presented at the Seventh International - International Building Performance Simulation Association (IBPSA) - Conference, Rio de Janeiro, Brazil, August 2001.

Deo, Narsingh. 1974. Graph Theory with Applications to Engineering and Computer Science. Saddle River, Prentice Hall.

Di Benedetto, M. and Isidori, A. 1978. Triangular canonical forms for bilinear systems. IEEE Transactions on Automatic Control 23, no.5: 877 - 880

Flouquet, F.O., and Kernevez, N. 1989. Study of the Performance of the Solar Heating System of a Multifamily Building. Energy and Buildings 13, no.2: 109-118.

Gilbert, Elmer G. 1963. Controllability and Observability in Multivariable Control Systems. . Journal of Society for Industrial and Applied Mathematics: Control 2, no.1: $128-151$.

Girard, A. and Hedrick, J. K. 2005. Control of Nonlinear Dynamic Systems: Theory and Applications. Lecture, Control of Nonlinear Dynamic Systems. University of California, Berekeley, CA. http://www.me.berkeley.edu/ME237/notes.html 
Glover, K. and Silverman, L. M. 1976. Characterization of Structural Controllability. IEEE Transactions on Automatic Control 21: 534-537.

Goodwine, Bill and McMickell, M. Brett. 2002. Reduction and Nonlinear Controllability of Symmetric Distributed Robotic Systems with Drift. Proceedings of the 2002 IEEE International Conference on Robotics and Automation 4: 3454-3460.

Grasselli, O. M. 1972. Controllability and Observability of Series Connections of Systems. Ricerche di Automatica 3: 44- 53.

Grasselli, O. M. and Isidori, A. 1977. Deterministic State Reconstruction and Reachability of Bilinear Processes. Proceedings of IEEE Joint Automatic Control Conference: 1423-1427. San Francisco, CA.

Hangos, K. M., Szigeti, F., and Varga, E. I. 1995. Controllability and Observability of Heat Exchanger Networks in the Time-Varying Parameter Case. Control Engineering Practice 3, no. 10: 1409-1419.

Hashimoto, Yukihiro, Kamimura, Kazuyuki, Kasahara, Masato, Kuzuu, Yoshiaki, and Matsuba, Tadahiko. 2000. Physical Model of an Air-Conditioned Space for Control Analysis. ASHRAE Transactions 106, no.2: 304-317. 
Hassan, Mohamed F. and Mahmoud, Magdi S. 1985. Large-Scale Control Systems: Theories and Techniques. Boca Raton: CRC Press

Haynes, G. W. and Hermes, H. 1970. Non-Linear Controllability via Lie-Theory. Society for Industrial and Applied Mathematics Journal: Control and Optimization 8: 450460.

Hermann, R. 1963. On the Accessibility problem in Control Theory. International Symposium of Nonlinear Differential Equation and Nonlinear Mechanics: 325332.

Hermann, Robert and Krener, Arthur J. 1977. IEEE Transactions on Automatic Control 22, no. 5: $728-740$

Hermes, H. 1964. Controllability and the Singular Problem. Journal of Society for Industrial and Applied Mathematics: Control 2, no.2: 241-260.

Ho, Y. C. and Mitter, S. K. 1976. Directions in Large Scale Systems. New York: Plenum Press.

Hwang, H.L. and Wolovich, W. A. 1974. Composite System Controllability and Observability. Automatica 10, no.2: 209-212. 
Isidori, Alberto. 1995. Nonlinear Control System. London: Springer-Verlag.

Ito, M. and Yonemura, Y. 1972. Controllability of composite systems of tandem connection. IEEE Transactions on Automatic Control 17, no. $\underline{5}$ : 722- 724.

James, M. R. 1987. Controllability and Observability of Nonlinear Systems. Tutorial. Mathematics Department and System Research Center, University of Maryland, College Park, MD.

Jamshidi, Mohammad. 1996. Large-Scale Systems: Modeling, Control and Fuzzy Logic. Englewood Cliff: Prentice-Hall PTR

Jurdjevic, Velimir. J. and Sussmann, Hector. J. 1972. Controllability of Nonlinear Systems. Journal of Differential Equations 12: 95-116.

Kailath, T. 1980. Linear Systems. Englewwod Cliffs, NJ: Prentice-Hall

Kalman, R. E., 1960. On the general theory of control system. Proceedings of First International Congress of Automatic Control, Moscow, USSR.

Kalman, R. E., Ho, Y. C., and Narendra, K.S. 1963. Controllability of linear dynamical system. Contr. Diff. Eqs. 1: $189-213$. 
Kalman, R. E.. 1960. Contributions to the theory of optimal control. Bol. Soc. Mat Mex. 5: $102-119$.

Kasinski, A. and Levine, J. 1984. A Fast Graph Theoretic Algorithm for the Feedback Decoupling Problem of Nonlinear Systems 58: 550-562. New York: Springer.

Khalil, Hasan. 1986. Modeling of Large-Scale Systems. In Singular perturbations in systems and control, ed. Petar V. Kokotovic and Hassan K. Khalil, 175. New York : IEEE Press.

Klamka, J. 1972. Uncontrollability and unobservability of multivariable systems. IEEE Transactions on Automatic Control 17, no. 5: 725- 726

Klamka, Jerzy. 1991. Controllability of Dynamical System. Norwell: Kluwer Academic Publishers.

Kodama, S. and Ohta, Y. 1985. Structural Invertibility of Transfer Functions. IEEE Transactions on Automatic Control 30: 818-819.

Kokotovic, Petar V. and Peponides, George. 1982. Singular Perturbations and Time Scales in Nonlinear Models of Power Systems. IEEE Transaction on Circuits and Systems 29, no. 11: 758-767. 
Kolodziej, W. J. and Mohler, R. R. 1980. An Overview of Bilinear System Theory and Applications. IEEE Transactions on Systems, Man, and Cybernetics 10, no. 10: 683-688.

Kreener, A. J. 1974. A Generalization of Chow's Theorem and the Bang Bang Theorem to Nonlinear Control Problems. Society for Industrial and Applied Mathematics Journal: Control and Optimization 12: 43-52.

Kreider, Jan, Curtiss, Peter, and Rabl, Ari. 1994. Heating and Cooling of Buildings: Design for Efficiency. Michigan: McGraw Hill.

Kucera, Jan. 1970. On Accessibility of Bilinear System. Czechoslovak Mathematical Journal 20, no. 95: 160-168.

Kuo, Benjamin C. 1995. Automatic Control Systems. Englewood Cliffs: Prentice Hall.

Lee, E. B. and Marcus, L. 1961. Optimal Control for Nonlinear Processes. Archive for Rational Mechanics and Analysis 8, no.11: 36-58.

Lee, E. B. and Marcus, L. 1967. Foundation of Optimal Control Theory. New York: Wiley 
Levine, J. 1997. A Graph-Theoretic Approach to Input Output Decoupling and Linearization. In Nonlinear Systems, ed. Fossard, A. J. and Normand-Cyrot, D. 77-91. London, UK: Chapman and Hall.

Li, Weiping and Slotine, Jean-Jacques E. 1990. Applied Nonlinear Control. Englewood Cliffs: Prentice Hall.

Lin, Ching-Tai. 1974. Structural Controllability. IEEE Transactions on Automatic Control 19, no.3: 201-208.

Ljung, Lennart. 1987. System Identification: Theory for the User. Englewood Cliffs, NJ: Prentice-Hall, Inc.

Luenberger, David G. 1964. Observing the State of a Linear System. IEEE Transactions on Military Electronics 8, no.2: 74-80.

Luenberger, David G. 1966. Observers for Multivariable Systems. IEEE Transactions on Automatic Control 11, no.2: 190-197.

Mahmoud, Magdi S. 1977. Multilevel Systems Control and Applications: A Survey. IEEE Transactions on Systems, Man and Cybernetics 7, no.3:125 - 143.

Mohler, R. R. 1973. Bilinear Control Process. New York: Academic. 
Mohler, R. R. and Rink, R. E. 1968. Completely Controllable Bilinear System. Journal of Society for Industrial and Applied Mathematics: Control 6: 477-486.

Mohler, R. R. and Shen, C. N. 1970. Optimal Control of Nuclear Reactors. New York: Academic.

Ore, Oystein. 1962. Theory of Graphs. American Mathematic Society Colloquium Publication 38.

Rabl, Ari. 1988. Parameter Estimation in Buildings: Methods for Dynamic Analysis of Measured Energy Use. Transactions of the ASME 110, no.2: 52-66.

Reinschke, Kurt J. 1984. Graph Theoretic Characterization of Structural Properties by Means of Paths and Cycle Families. IFAC World Congress, Budapest

Reinschke, Kurt J. 1988. Multivariable Control, A Graph Theoretic Approach. New York: Springer.

Rosenbrock, H. H. 1970. State-Space and Multivariable Theory. New York: Wiley.

Sen P. 1981. On the Choice of Input for Observability in Bilinear Systems. IEEE Transactions on Automatic Control 26, no. 2: 451-454. 
Shields, R. W. and Pearson, J. B. 1976. Structural Controllability of Multi-InputLinear System. IEEE Transactions on Automatic Control 21: 203-212.

Siljak, Dragoslav D. 1977. On Reachability of Dynamic Systems. International Journal of System Science 8, no. 3: 321-338.

Siljak, Dragoslav D. 1978. Large-Scale Dynamic Systems: Stability and Structure. New York: North-Holland.

Siljak, Dragoslav D. 1999. Large-Scale and Decentralized Systems. In Wiley Encyclopedia of Electrical and Electronics Engineering 11, ed. John G. Webster, 209-224. New York: John Wiley \& Sons, Inc.

Siljak, Dragoslav D. 2005. Control of Large-Scale Systems: Beyond Decentralized Feedback. Annual Reviews in Control 29, no. 2: 169-179.

Siljak, Dragoslav D., and Vukcevic, M.B. 1977. Decentrally Stabilizable Linear and Bilinear Large-Scale Systems. International Journal of Control 26, no. 2: 289305.

Strogatz, Steven H. 2001. Nonlinear Dynamics And Chaos: With Applications To Physics, Biology, Chemistry, And Engineering. Boulder: Westview Press. 
Sussmann, H. J. 1987. A General Theorem on Local Controllability. Society for Industrial and Applied Mathematics Journal: Control and Optimization 25, no.1: 158-194.

Sussmann, Hector. J. 1983. Lie Brackets, Real Analyticity and Geometric Control. In Differential Geometric Control Theory, ed. Brockett, R. W., Millman R. S., and Sussmann, H. J. 1-116. Boston, MA: Birkhauser.

Tamura, Hiroyuki and Yoshikawa, Tsuneo. 1990. Large-Scale Systems Control and Decision Making. New York: Marcel Dekker.

Van der Woude, Jacob W. 1991. A Graph Theoretic Characterization for the Rank of the Transfer Matrix of a Structured System. Mathematics of Control, Signals and Systems 4: 33-40.

Vidyasagar, M. 1980. Decomposition Techniques for Large-Scale Systems with Nonadditive Interactions: Stability and Stabilizability. IEEE Transactions on Automatic Control 25, no. 4: 773-779.

Williamson, D. 1977. Observation of Bilinear Systems with Application to Biological Control. Automatica 13, no.3: 243-254. 
Wonham, W. M. 1985. Linear Multivariable Control: A Geometric Approach. Berlin: Springer 


\section{Appendix 1}

\section{Heat Exchanger Model:}

Controllability and Observability of a Thermal Dynamic Nonlinear System 


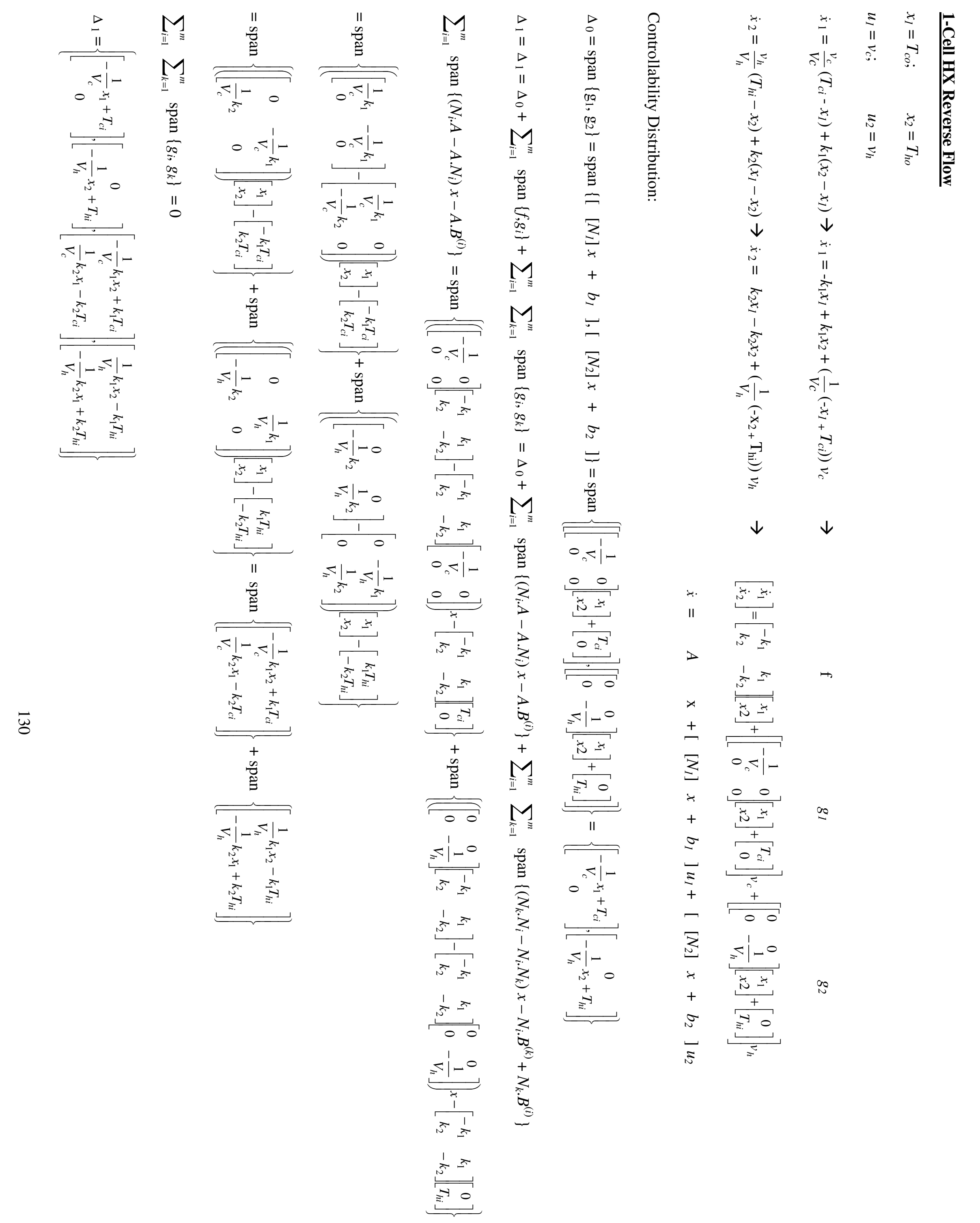




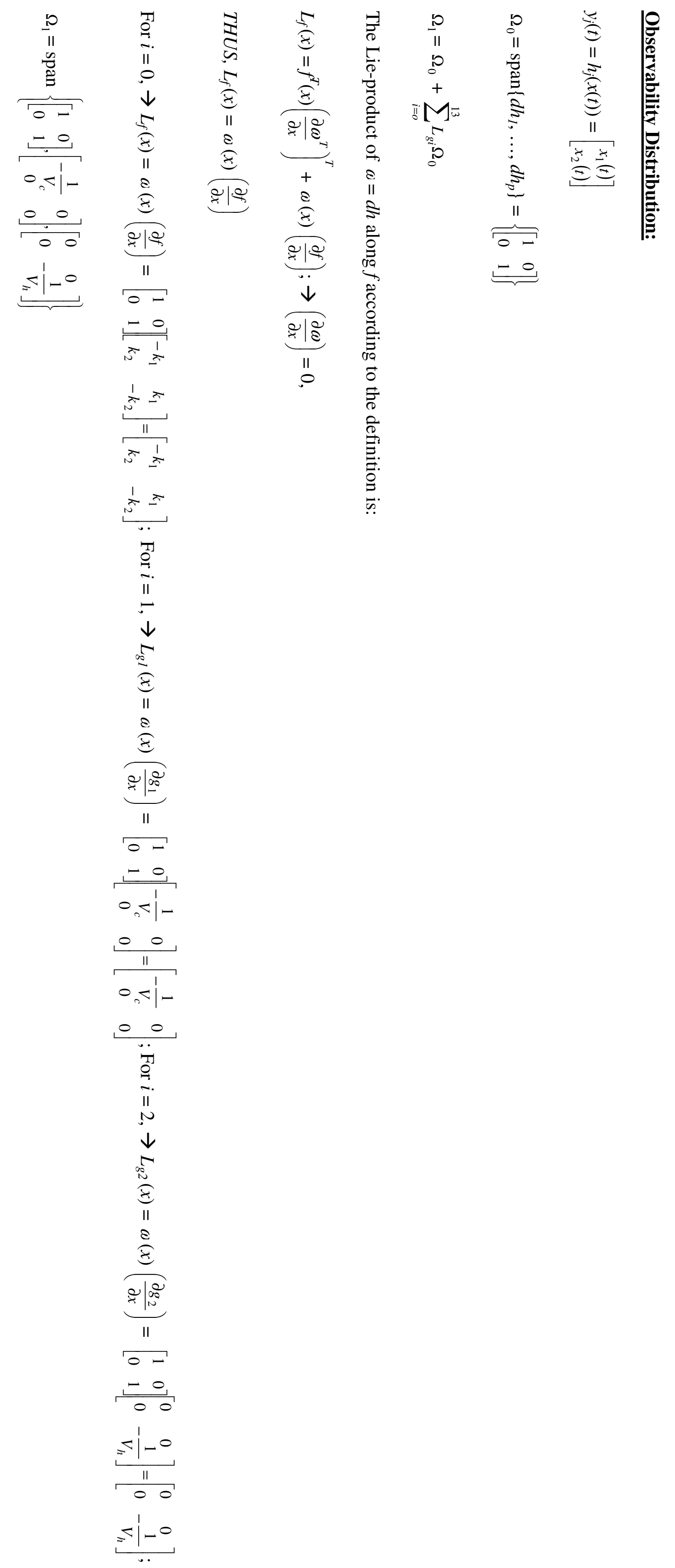



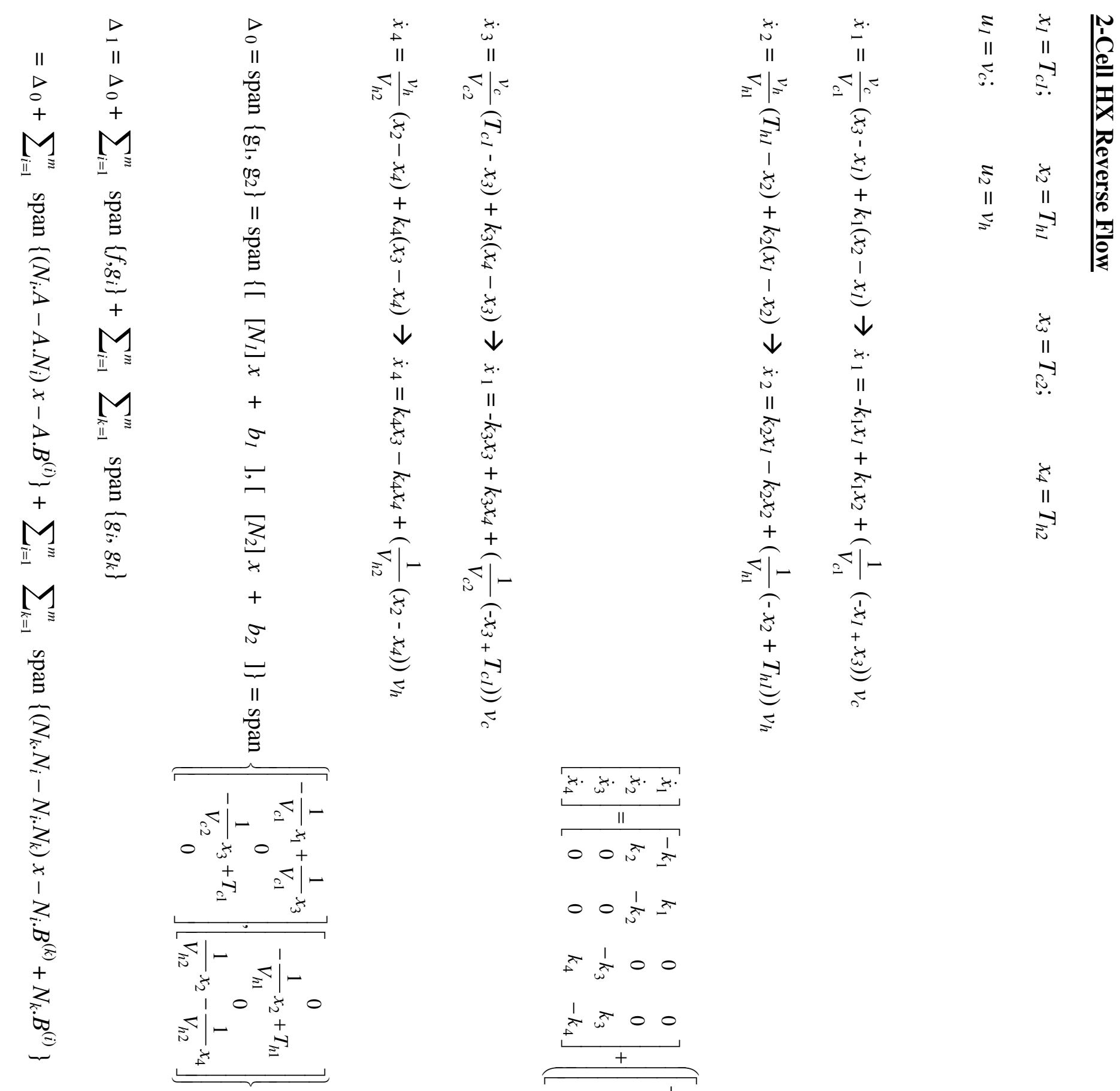

0000

$\left.0_{i}\right|_{1}-0_{2} \leq \mid-$

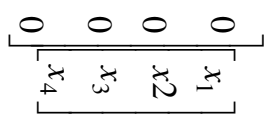
$\frac{1}{0} 00$

$\frac{+}{0000}$

离|-

0000

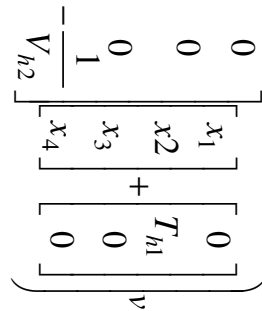




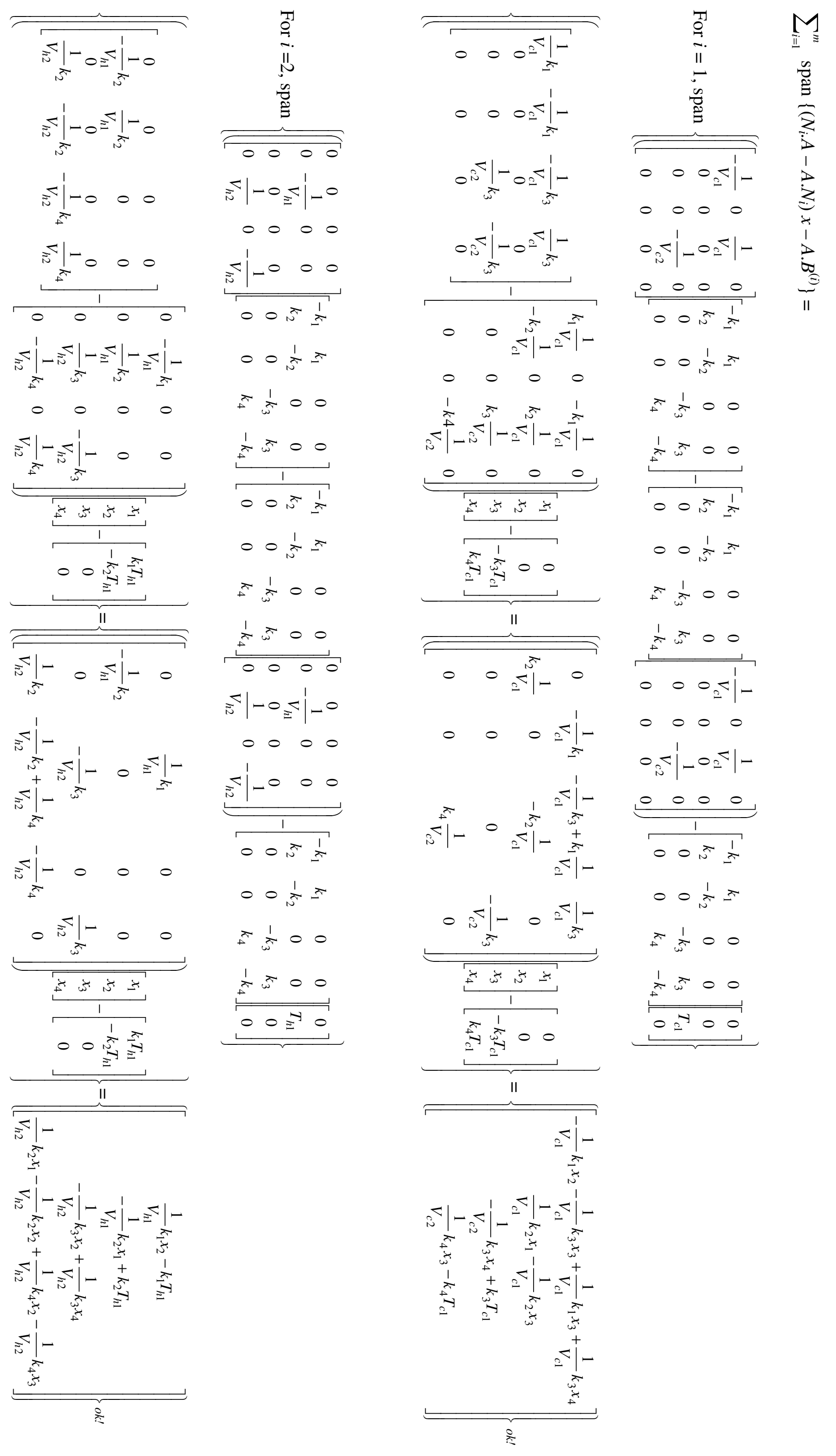




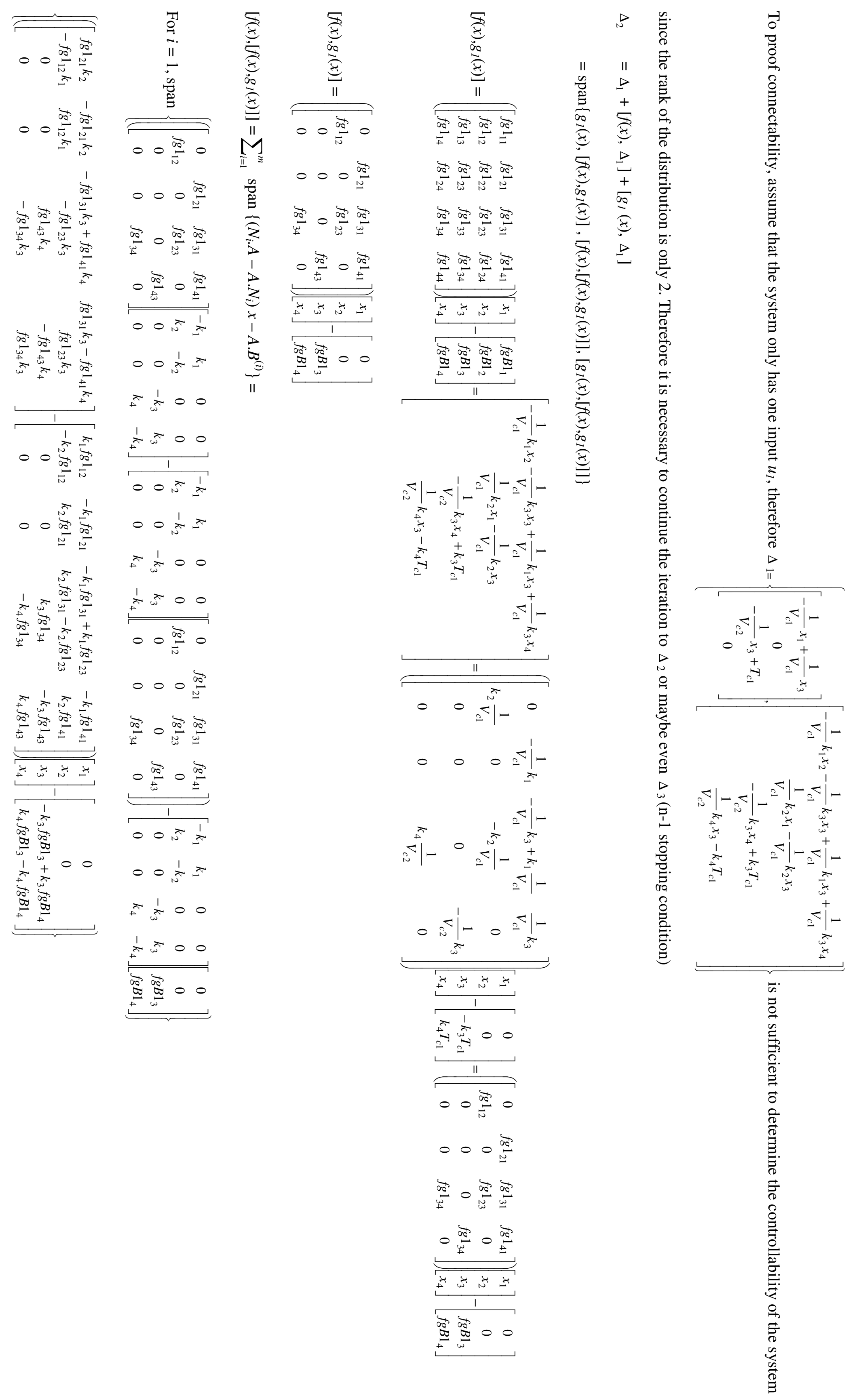




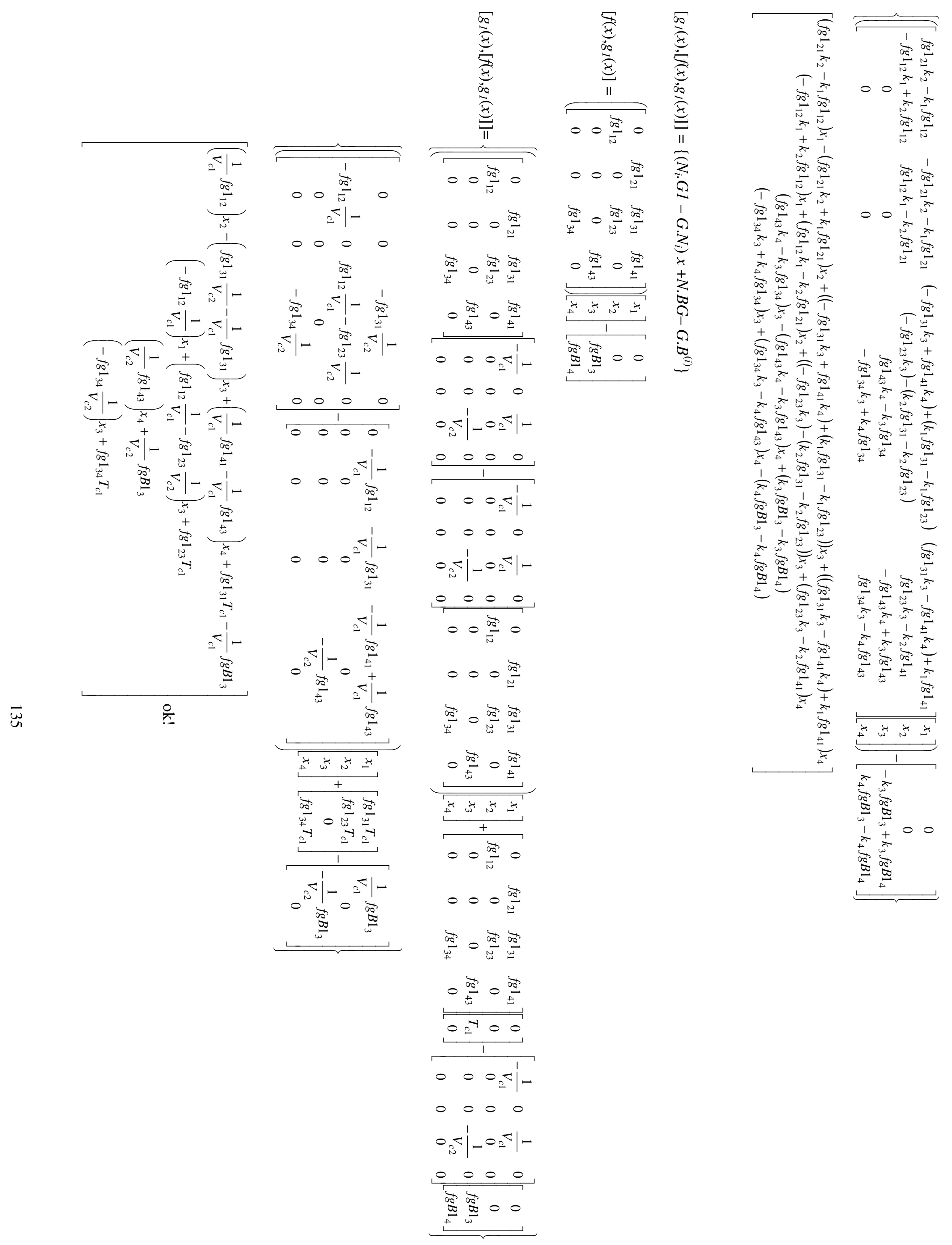




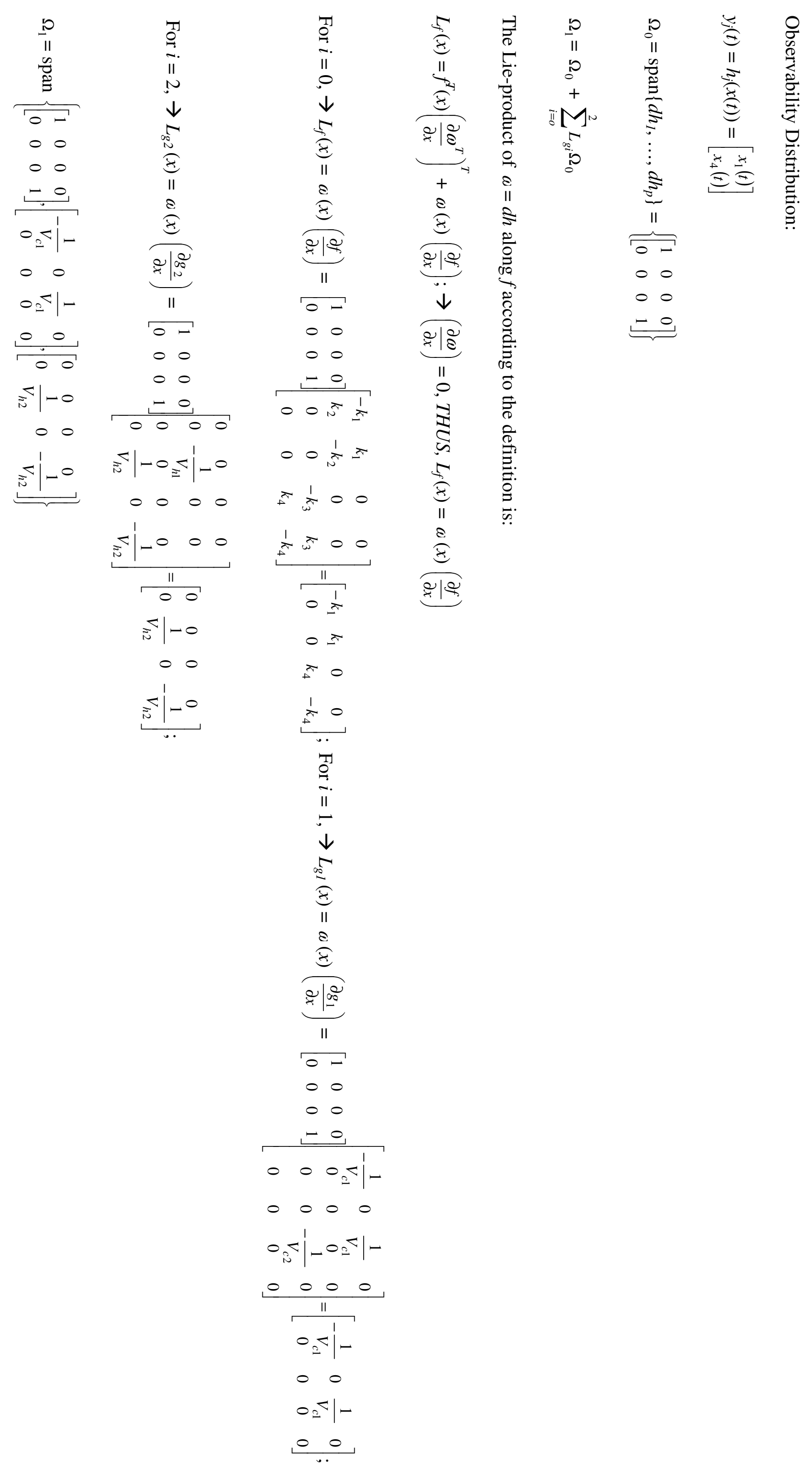


Appendix 2

BCHP Model 


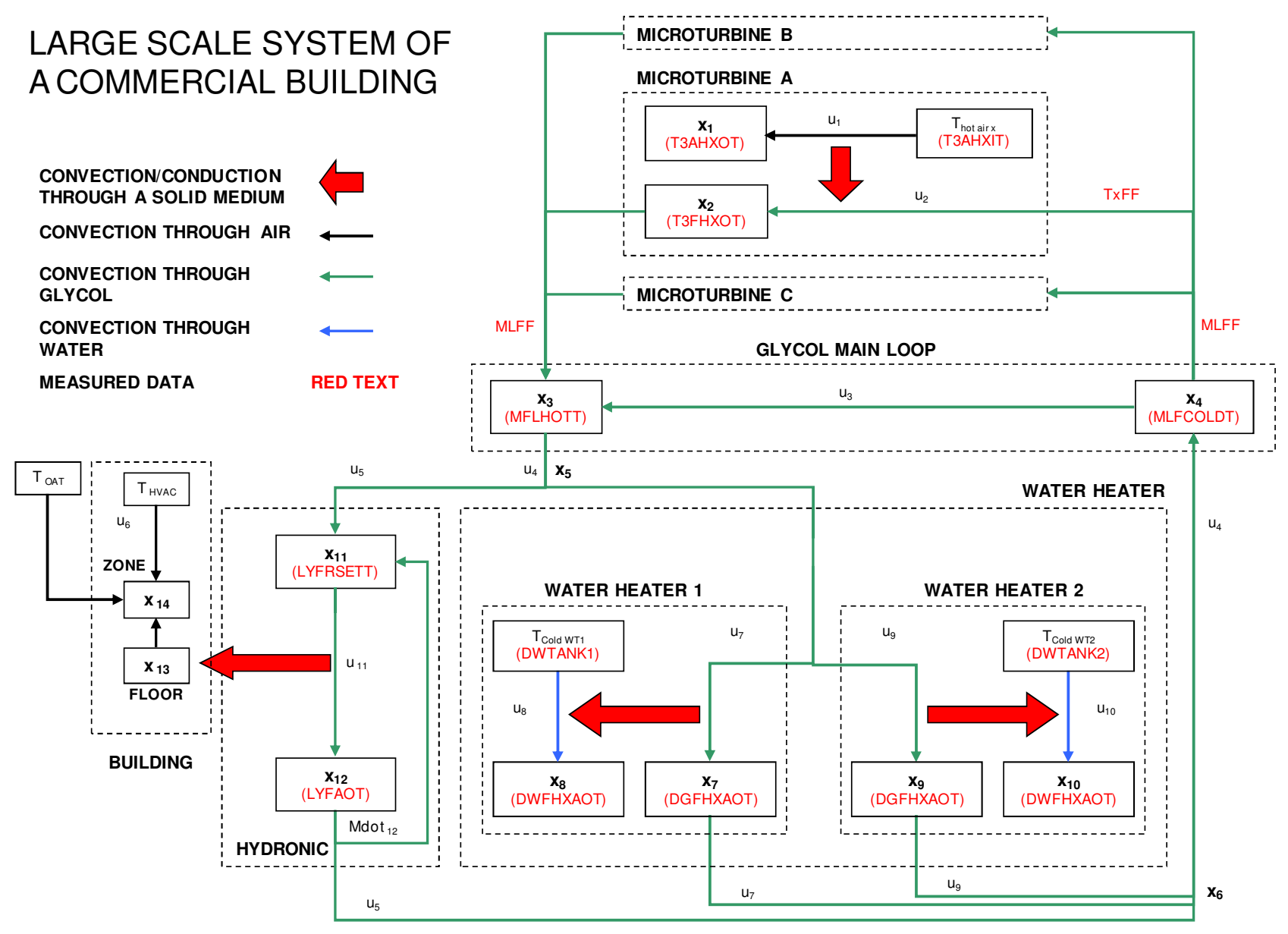

Figure 29: BCHP System and Subsystem Boundary 
Table 10: State Notation

\begin{tabular}{|l|c|}
\hline \multicolumn{1}{|c|}{ Definition } & $\begin{array}{c}\text { Large Scale } \\
\text { System Level }\end{array}$ \\
\hline Microturbine-HX Air Supply temperature & $x_{1}$ \\
\hline Microturbine-HX Glycol Supply temperature & $x_{2}$ \\
\hline Main Loop-HX Primary Glycol Supply temperature & $x_{3}$ \\
\hline Main Loop-HX Primary Glycol Return temperature & $x_{4}$ \\
\hline Main Loop Glycol Secondary Supply temperature & $x_{5}$ \\
\hline Main Loop Glycol Secondary Return temperature & $x_{6}$ \\
\hline DWH-HX "A" - Glycol Supply temperature & $x_{7}$ \\
\hline DWH-HX "A" - Water Return temperature & $x_{8}$ \\
\hline DWH-HX "B" - Glycol Supply temperature & $x_{9}$ \\
\hline DWH-HX "B" - Water Return temperature & $x_{10}$ \\
\hline Hydronic - Glycol Supply temperature & $x_{11}$ \\
\hline Hydronic - Glycol Return temperature & $x_{12}$ \\
\hline Floor temperature & $x_{13}$ \\
\hline Building Space Air temperature & $x_{14}$ \\
\hline
\end{tabular}

Table 11: Constant Input

\begin{tabular}{|l|c|}
\hline \multicolumn{1}{|c|}{ Definition } & Large-Scale System Notation \\
\hline Microturbine-HX air inlet temperature & $b_{1}$ \\
\hline DWH-HX "A" water inlet temperature & $b_{2}$ \\
\hline DWH-HX "B" water inlet temperature & $b_{3}$ \\
\hline HVAC air supply temperature & $b_{4}$ \\
\hline
\end{tabular}


Table 12: Controllable Input

\begin{tabular}{|l|c|}
\hline \multicolumn{1}{|c|}{ Definition } & $\begin{array}{c}\text { Large-Scale System } \\
\text { Notation }\end{array}$ \\
\hline Microturbine-HX exhaust air volumetric flow rate & $u_{1}$ \\
\hline Microturbine-HX glycol volumetric flow rate & $u_{2}$ \\
\hline Main loop glycol bypass volumetric flow rate & $u_{3}$ \\
\hline Main loop glycol volumetric flow rate & $u_{4}$ \\
\hline Hydronic glycol volumetric flow rate & $u_{5}$ \\
\hline HVAC supply air volumetric flow rate & $u_{6}$ \\
\hline DWH-HX "A" glycol volumetric flow rate & $u_{7}$ \\
\hline DWH-HX "A" water volumetric flow rate & $u_{8}$ \\
\hline DWH-HX "B" glycol volumetric flow rate & $u_{9}$ \\
\hline DWH-HX "B" water volumetric flow rate & $u_{10}$ \\
\hline Hydronic glycol mixed volumetric flow rate & $u_{11}$ \\
\hline Hydronic glycol return volumetric flow rate & $u_{12}$ \\
\hline
\end{tabular}




\section{HX at the Microturbine}

Equations 1 and 2 are the differential equations that govern the heat exchanger between the hot exhaust-air from a micorturbine to the glycol main loop unit, which is based on the conservation of energy law. In linear system the input is the temperature of hot air that is produced by the microturbine. However in a nonlinear system, which is always the case, the input is the volume flow rate of the hot air that is coming from the microturbine as well as the volume flow rate of glycol that is coming from glycol main loop.

1. $\quad \dot{u}_{1} c_{1}\left(b_{1}-x_{1}\right)=\rho_{1} c_{1} V_{1} \frac{\partial x_{1}}{\partial t}+\frac{\left(x_{1}-x_{2}\right)}{R_{1-2}} \rightarrow \frac{\partial x_{1}}{\partial t}=-\frac{\left(-x_{1}+x_{2}\right)}{\rho_{1} c_{1} V_{1} R_{1-2}}+\frac{\dot{u}_{1} c_{1}\left(b_{1}-x_{1}\right)}{\rho_{1} c_{1} V_{1}}=-\left(k_{1}\right) x_{1}+\left(k_{1}\right) x_{2}+\frac{\left(-x_{1}+b_{1}\right)}{V_{1}} u_{1}$

$\frac{\partial x_{1}}{\partial t}=-\left(k_{1}\right) x_{1}+\left(k_{1}\right) x_{2}+\frac{\left(-x_{1}+b_{1}\right)}{V_{1}} u_{1}$

$y_{1}=x_{1}$

2. $\frac{\left(x_{1}-x_{2}\right)}{R_{2-1}}+\dot{u}_{2} c_{4}\left(x_{4}-x_{2}\right)=\rho_{2} c_{2} V_{2} \frac{\partial x_{2}}{\partial t} \rightarrow \frac{\partial x_{2}}{\partial t}=\frac{\left(x_{1}-x_{2}\right)}{\rho_{2} c_{2} V_{2} R_{2-1}}+\frac{\dot{u}_{2} c_{4}\left(x_{4}-x_{2}\right)}{\rho_{2} c_{2} V_{2}}=\left(k_{2}\right) x_{1}-\left(k_{2}\right) x_{2}+\frac{\left(-x_{2}+x_{4}\right)}{V_{2}} u_{2}$

$\frac{\partial x_{2}}{\partial t}=\left(k_{2}\right) x_{1}-\left(k_{2}\right) x_{2}+\frac{\left(-x_{2}+x_{4}\right)}{V_{2}} u_{2}$

$y_{2}=x_{2}$ 


\section{Glycol Loop}

Glycol main loop is the system that distributes the thermal energy that is produced by the microturbines to the load in the building.

Glycol is the fluid that is used for the system. In this system, it is assumed that the thermal property of the glycol stays constant all through the process. Equations 3 through 6 are the differential equations of the glycol main loop, which is based on the law of conservation of energy. Equation 3 and 4 represent the dynamic of the glycol at the microturbine plant or in this case is called primary loop. Equation 5 and 6 represent the dynamic of the glycol at the load level or in this case is called secondary loop.

3. $\dot{u}_{3} c_{4}\left(x_{4}-x_{3}\right)+\dot{u}_{2} c_{2}\left(x_{2}-x_{3}\right)=\rho_{3} c_{3} V_{3} \frac{\partial x_{3}}{\partial t} \rightarrow \frac{\partial x_{3}}{\partial t}=\frac{\dot{u}_{2} c_{2}\left(x_{2}-x_{3}\right)}{\rho_{3} c_{3} V_{3}}+\frac{\dot{u}_{3} c_{3}\left(x_{4}-x_{3}\right)}{\rho_{3} c_{3} V_{3}}=\frac{\left(x_{2}-x_{3}\right)}{V_{3}} u_{2}+\frac{\left(-x_{3}+x_{4}\right)}{V_{3}} u_{3}$ $y_{3}=x_{3}$

4. $\quad \dot{u}_{4} c_{6}\left(x_{6}-x_{4}\right)=\rho_{4} c_{4} V_{4} \frac{\partial x_{4}}{\partial t} \rightarrow \frac{\partial x_{4}}{\partial t}=\frac{u_{4} c_{6}\left(x_{6}-x_{4}\right)}{\rho_{4} c_{4} V_{4}}=\frac{\left(x_{6}-x_{4}\right)}{V_{4}} u_{4}$ $\frac{\partial x_{4}}{\partial t}=\frac{\left(x_{6}-x_{4}\right)}{V_{4}} u_{4}$

$y_{4}=x_{4}$ 
5. $\quad \dot{u}_{4} c_{3}\left(x_{3}-x_{5}\right)=\rho_{5} c_{5} V_{5} \frac{\partial x_{5}}{\partial t} \rightarrow \frac{\partial x_{5}}{\partial t}=\frac{\dot{u}_{4} c_{5}\left(x_{3}-x_{5}\right)}{\rho_{5} c_{5} V_{5}}=\frac{\left(x_{3}-x_{5}\right)}{V_{5}} u_{4}$

$\frac{\partial x_{5}}{\partial t}=\frac{\left(x_{3}-x_{5}\right)}{V_{5}} u_{4}$

$y_{5}=x_{5}$

6. $\quad \dot{u}_{7} c_{7}\left(x_{7}-x_{6}\right)+\dot{u}_{9} c_{9}\left(x_{9}-x_{6}\right)+\dot{u}_{5} c_{12}\left(x_{12}-x_{6}\right)=\rho_{6} c_{6} V_{6} \frac{\partial x_{6}}{\partial t} \rightarrow \frac{\partial x_{6}}{\partial t}=\frac{\left(x_{7}-x_{6}\right)}{V_{6}} u_{7}+\frac{\left(x_{9}-x_{6}\right)}{V_{6}} u_{9}+\frac{\left(x_{12}-x_{6}\right)}{V_{6}} u_{5}$

$\frac{\partial x_{6}}{\partial t}=\frac{\left(x_{7}-x_{6}\right)}{V_{6}} u_{7}+\frac{\left(x_{9}-x_{6}\right)}{V_{6}} u_{9}+\frac{\left(x_{12}-x_{6}\right)}{V_{6}} u_{5}$

$y_{6}=x_{6}$ 


\section{$\underline{\text { Water Heater }}$}

There are two 1000 gallon water tanks that its temperature is required to maintain at $145^{\circ} \mathrm{F}$ at all times. Equations 7 through 10 are the differential equations to govern the dynamic of these two water tanks. Equations 7 and 8 represent the system for tank 1 and equations 9 and 10 represent the system for tank 2. There are two operation modes for each tank namely charging and not-charging. Charging occurs when the temperature of the water inside the tank is less than $145^{0} \mathrm{~F}$. When the temperature of the water in the tanks is equal or greater than $145^{\circ} \mathrm{F}$, the flow from the hot glycol to the hot water heat exchanger is shut down.

7(9).

$$
\begin{aligned}
& \dot{u}_{7} c_{5}\left(x_{5}-x_{7}\right)=\rho_{7} c_{7} V_{7} \frac{\partial x_{7}}{\partial t}+\frac{\left(x_{7}-x_{8}\right)}{R_{7-8}} \rightarrow \frac{\partial x_{7}}{\partial t}=-\frac{\left(x_{7}-x_{8}\right)}{\rho_{7} c_{7} V_{7} R_{7-8}}+\frac{\dot{u}_{7} c_{5}\left(x_{5}-x_{7}\right)}{\rho_{7} c_{7} V_{7}}= \\
& \frac{\partial x_{7}}{\partial t}-\frac{1}{\rho_{7} c_{7} V_{7} R_{7-8}} x_{7}+\frac{1}{\rho_{7} c_{7} V_{7} R_{7-8}} x_{8}+\frac{\left(x_{5}-x_{7}\right)}{V_{7}} u_{7}=-k_{7} x_{7}+k_{7} x_{8}+\frac{\left(x_{5}-x_{7}\right)}{V_{7}} u_{7}
\end{aligned}
$$

8(10). $\quad \dot{u}_{8} c_{8}\left(b_{2}-x_{8}\right)=\rho_{8} c_{8} V_{8} \frac{\partial x_{8}}{\partial t}+\frac{\left(x_{8}-x_{7}\right)}{R_{8-7}} \rightarrow \frac{\partial x_{8}}{\partial t}=-\frac{\left(x_{7}-x_{8}\right)}{\rho_{8} c_{8} V_{8} R_{8-7}}+\frac{\dot{u}_{8} c_{8}\left(b_{2}-x_{8}\right)}{\rho_{8} c_{8} V_{8}}=$

$$
\frac{\partial x_{8}}{\partial t}=-\frac{1}{\rho_{8} c_{8} V_{8} R_{8-7}} x_{7}+\frac{1}{\rho_{8} c_{8} V_{8} R_{8-7}} x_{8}+\frac{\left(b_{2}-x_{8}\right)}{V_{8}} u_{8}=-k_{8} x_{7}+k_{8} x_{8}+\frac{\left(b_{2}-x_{8}\right)}{V_{8}} u_{8}
$$

$y_{7(9)}=x_{7(9) \text { and }} y_{8(10)}=x_{8(10)}$ 


\section{Hydronic $(11,12,13,14)$}

Hydronic Radiant Floor heating system is one of the building equipment that is used to maintain the temperature of building interior at the comfortable level during winter. Hydronic system consists of circuits of tubular pipe that is embedded under the floor. The pipe carries heated glycol from the glycol main loop, which has a function to heat the floor through conduction and convection. The heated floor then would radiate the heat to the interior of the building. Equations 11 through 14 are the differential equations of the hydronic system, which is based on the law of conservation of energy. In the case of this project, the hydronic system is controlled by regulating the flow rate of the glycol underneath the floor in order to maintain its temperature at $105^{\circ} \mathrm{F}$ during winter and $85^{\circ} \mathrm{F}$ during summer. This is achieved by mixing the glycol supply and its return. This process is represented by Equation 11 and 12 . The load side of the hydronic system, which is at the building level, is represented by Equation 13 and 14 which shows the interaction that occurs in the building zone between the heated floor (hydronic system) and the building load, as well as additional heating system that is contributed by conventional HVAC system. 
11. $\dot{u}_{5} c_{5}\left(x_{5}-x_{11}\right)+\dot{u}_{12} c_{12}\left(x_{12}-x_{11}\right)=\rho_{11} c_{11} V_{11} \frac{\partial x_{11}}{\partial t} \rightarrow \frac{\partial x_{11}}{\partial t}=\frac{\dot{u}_{5} c_{5}\left(x_{5}-x_{11}\right)}{\rho_{11} c_{11} V_{11}}+\frac{\dot{u}_{12} c_{12}\left(x_{12}-x_{11}\right)}{\rho_{11} c_{11} V_{11}}$

$$
\frac{\partial x_{11}}{\partial t}=\frac{\left(x_{5}-x_{11}\right)}{V_{11}} u_{5}+\frac{\left(x_{12}-x_{11}\right)}{V_{11}} u_{12}=\frac{\left(x_{5}-x_{11}\right)}{V_{11}} u_{5}+\frac{\left(x_{12}-x_{11}\right)}{V_{11}} u_{12}
$$

$y_{11}=x_{11}$

12. $\dot{u}_{11} c_{11}\left(x_{11}-x_{12}\right)=\rho_{12} c_{12} V_{12} \frac{\partial x_{12}}{\partial t} \rightarrow \frac{\partial x_{12}}{\partial t}=\frac{\dot{u}_{11} c_{11}\left(x_{11}-x_{12}\right)}{\rho_{12} c_{12} V_{12}}=\frac{\left(x_{11}-x_{12}\right)}{V_{12}} u_{11}$

$$
\frac{\partial x_{12}}{\partial t}=\frac{\left(x_{11}-x_{12}\right)}{V_{12}} u_{11}
$$

$y_{12}=x_{12}$

13. $\dot{u}_{11} c_{12}\left(x_{12}-x_{13}\right)=\rho_{13} c_{13} V_{13} \frac{\partial x_{13}}{\partial t}+\frac{\left(x_{13}-x_{14}\right)}{R_{13-14}} \rightarrow \frac{\partial x_{13}}{\partial t}=-\frac{\left(x_{13}-x_{14}\right)}{\rho_{13} c_{13} V_{13} R_{13-14}}+\frac{\dot{u}_{11} c_{12}\left(x_{12}-x_{13}\right)}{\rho_{13} c_{13} V_{13}}=$

$$
\frac{\partial x_{13}}{\partial t}==-\frac{1}{\rho_{13} c_{13} V_{13} R_{13-14}} x_{13}+\frac{1}{\rho_{13} c_{13} V_{13} R_{13-14}} x_{14}+\frac{\left(-x_{13}+x_{12}\right)}{V_{13}} u_{11}=-\left(k_{13}\right) x_{13}+\left(k_{13}\right) x_{14}+\frac{\left(-x_{13}+x_{12}\right)}{V_{13}} u_{11}
$$

$$
y_{13}=x_{13}
$$


14. $\frac{\left(x_{13}-x_{14}\right)}{R_{14-13}}+\dot{u}_{6} c_{14}\left(b_{4}-x_{14}\right)=\rho_{14} c_{14} V_{14} \frac{\partial x_{14}}{\partial t} \rightarrow \frac{\partial x_{14}}{\partial t}=\frac{\left(x_{13}-x_{14}\right)}{\rho_{14} c_{14} V_{14} R_{14-13}}+\frac{\dot{u}_{6} c_{14}\left(b_{4}-x_{14}\right)}{\rho_{14} c_{14} V_{14}}=$

$$
\frac{\partial x_{14}}{\partial t}=\frac{1}{\rho_{14} c_{14} V_{14} R_{14-13}} x_{13}-\frac{1}{\rho_{14} c_{14} V_{14} R_{14-13}} x_{14}+\frac{\left(b_{4}-x_{14}\right)}{V_{14}} u_{6}=\left(k_{14}\right) x_{13}+-\left(k_{14}\right) x_{14}+\frac{\left(-x_{14}+b_{4}\right)}{V_{14}} u_{6}
$$

$$
y_{14}=x_{14}
$$

The state space form of the nonlinear large scale model of BCHP follows the following equation:

State Equation:

$$
\dot{x}=f(x)+g(x)=f(x)+\sum_{i=1}^{m} g_{i}(x)=A_{\text {Transfer }}(x)+\sum_{i=1}^{m} N_{i}(x)+B^{(i)} \text { Convection }
$$

Output Equation: $\quad y_{j}(t)=h_{j}(x(t)), j=1, \ldots, p$ 


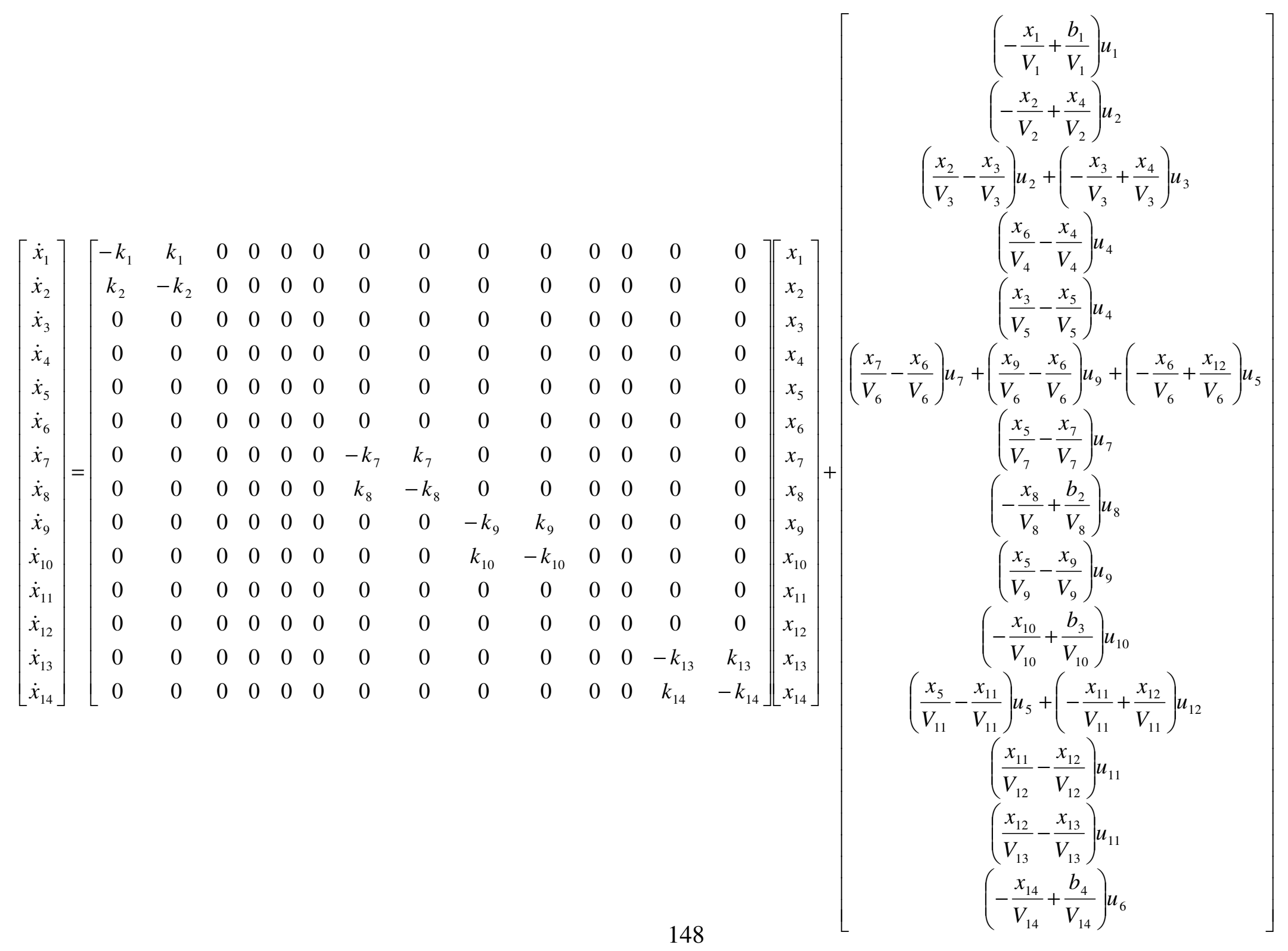




$$
y(t)=\left[\begin{array}{c}
y_{1} \\
y_{2} \\
\vdots \\
y_{14}
\end{array}\right]=I \cdot\left[\begin{array}{c}
x_{1} \\
x_{2} \\
\vdots \\
x_{14}
\end{array}\right] \text {, where } I \text { is a } 14 \times 14 \text { matrix identity }
$$


Appendix 3

Controllability of a Nonlinear System: A Proof 


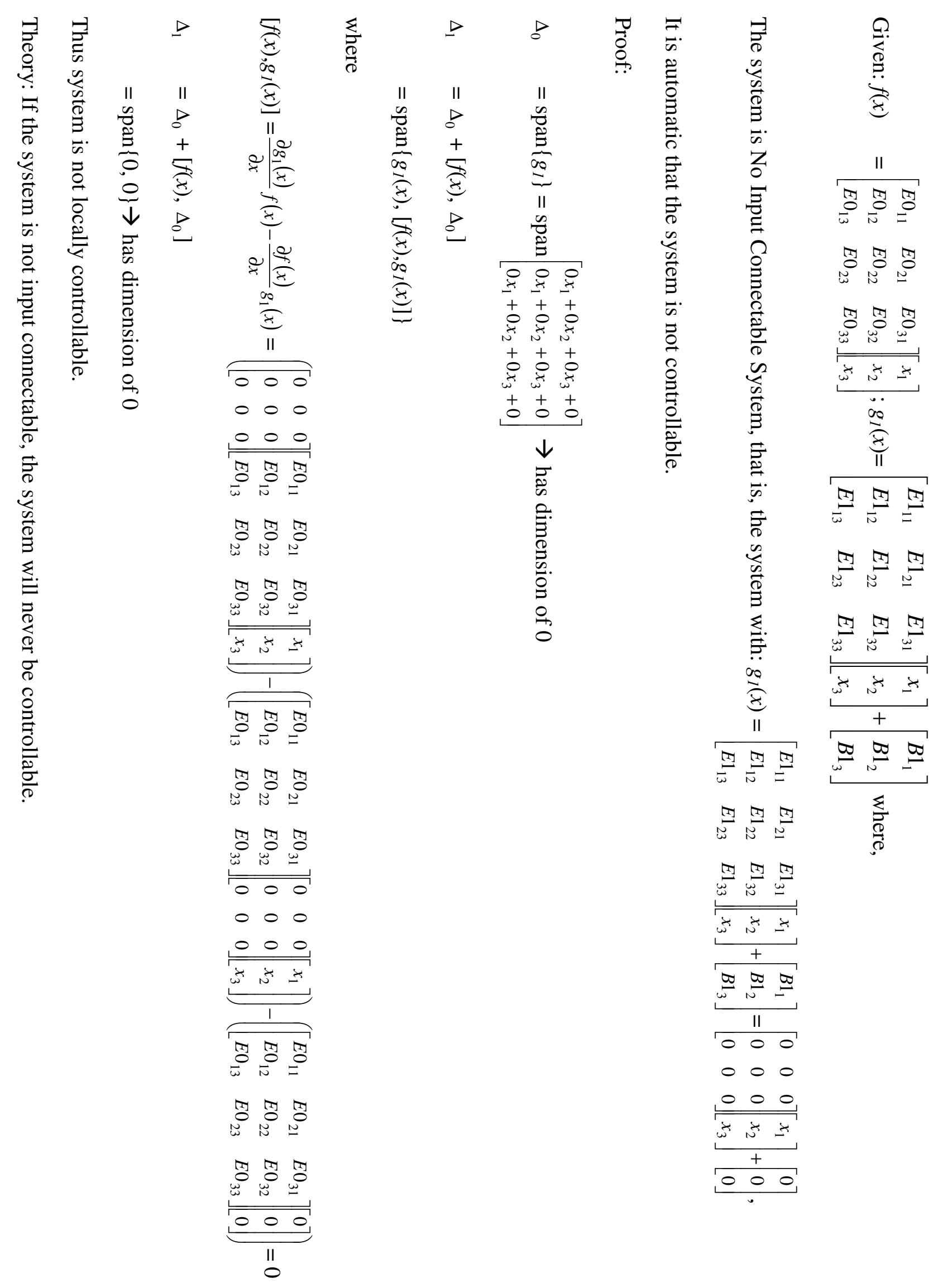



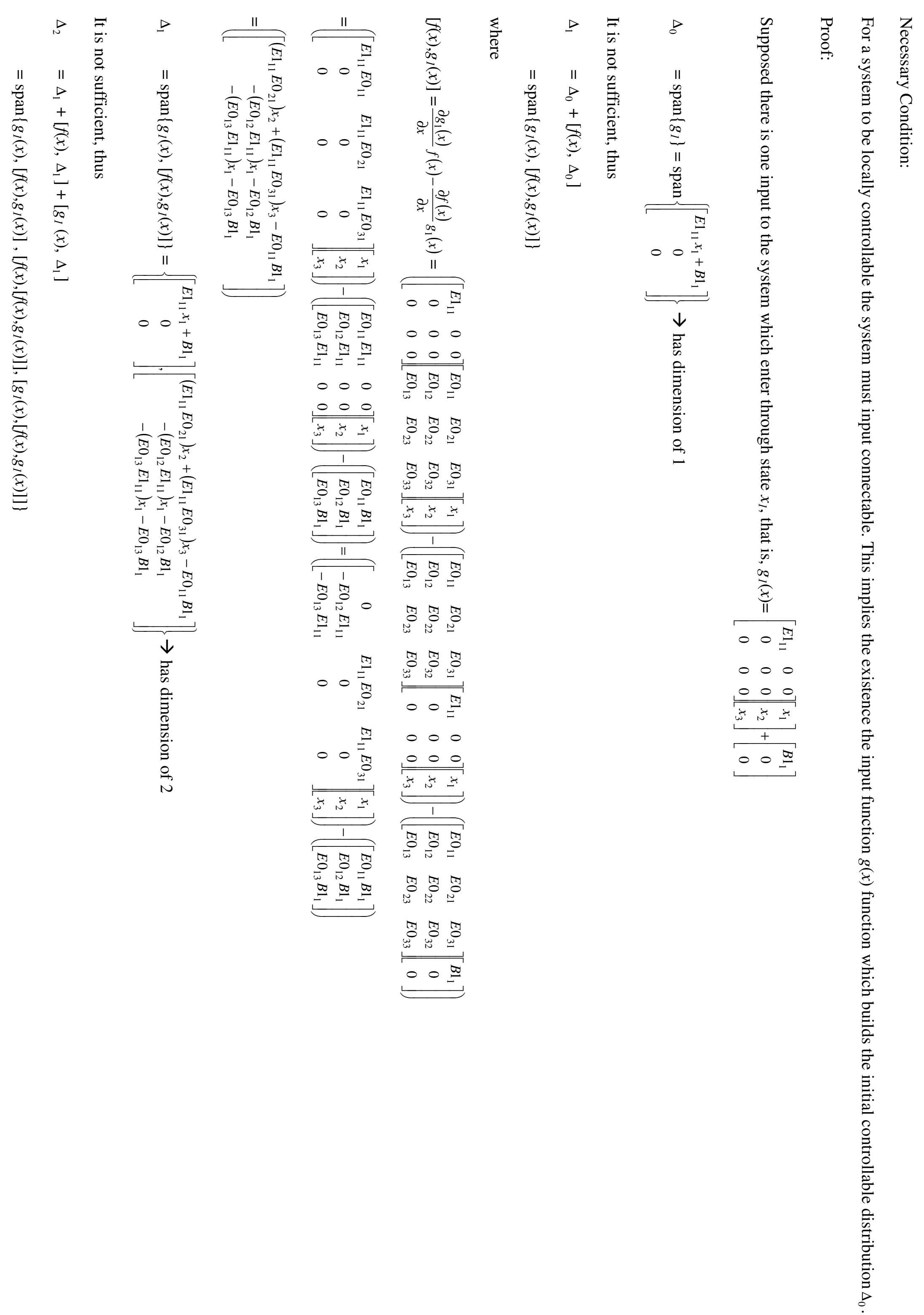


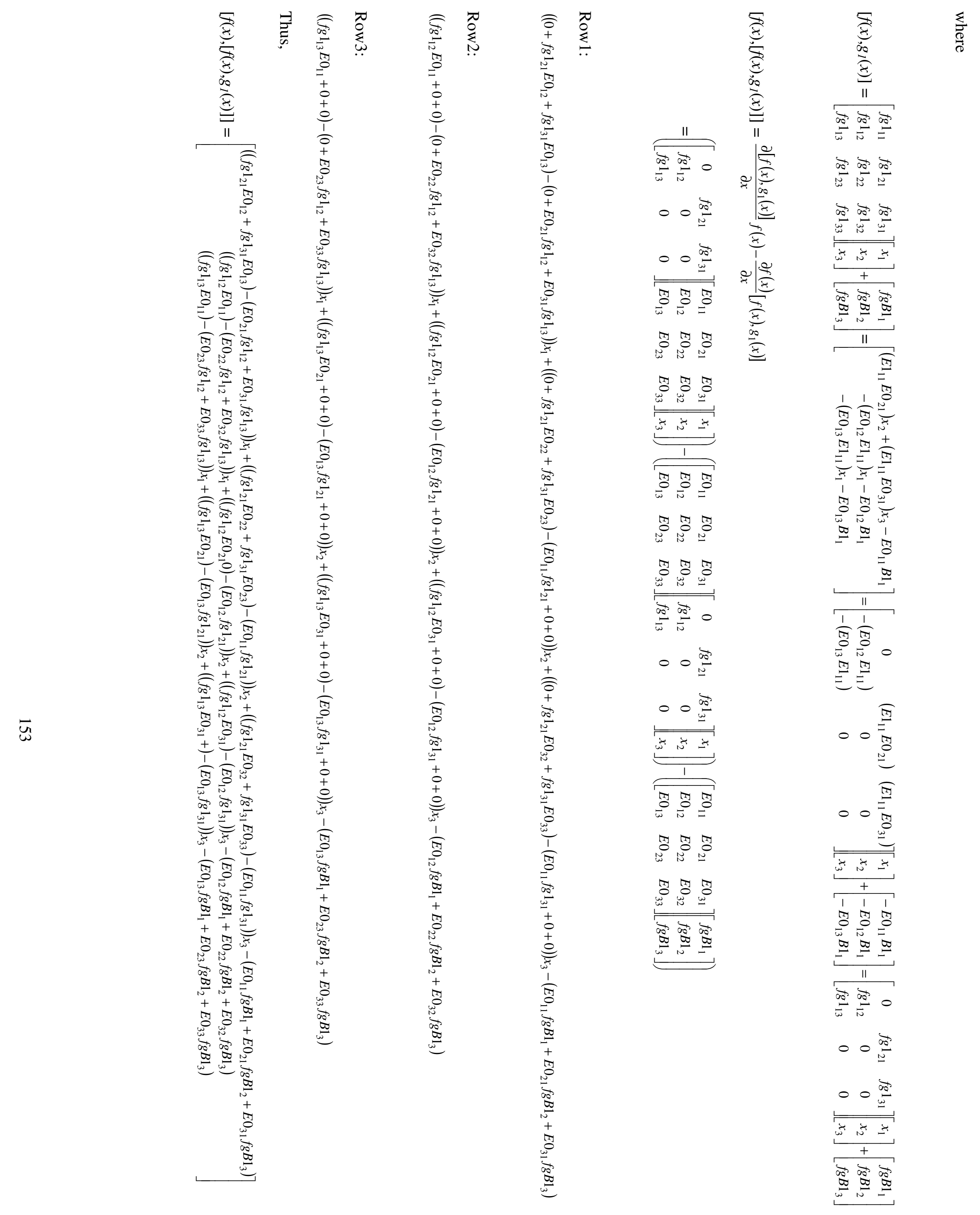




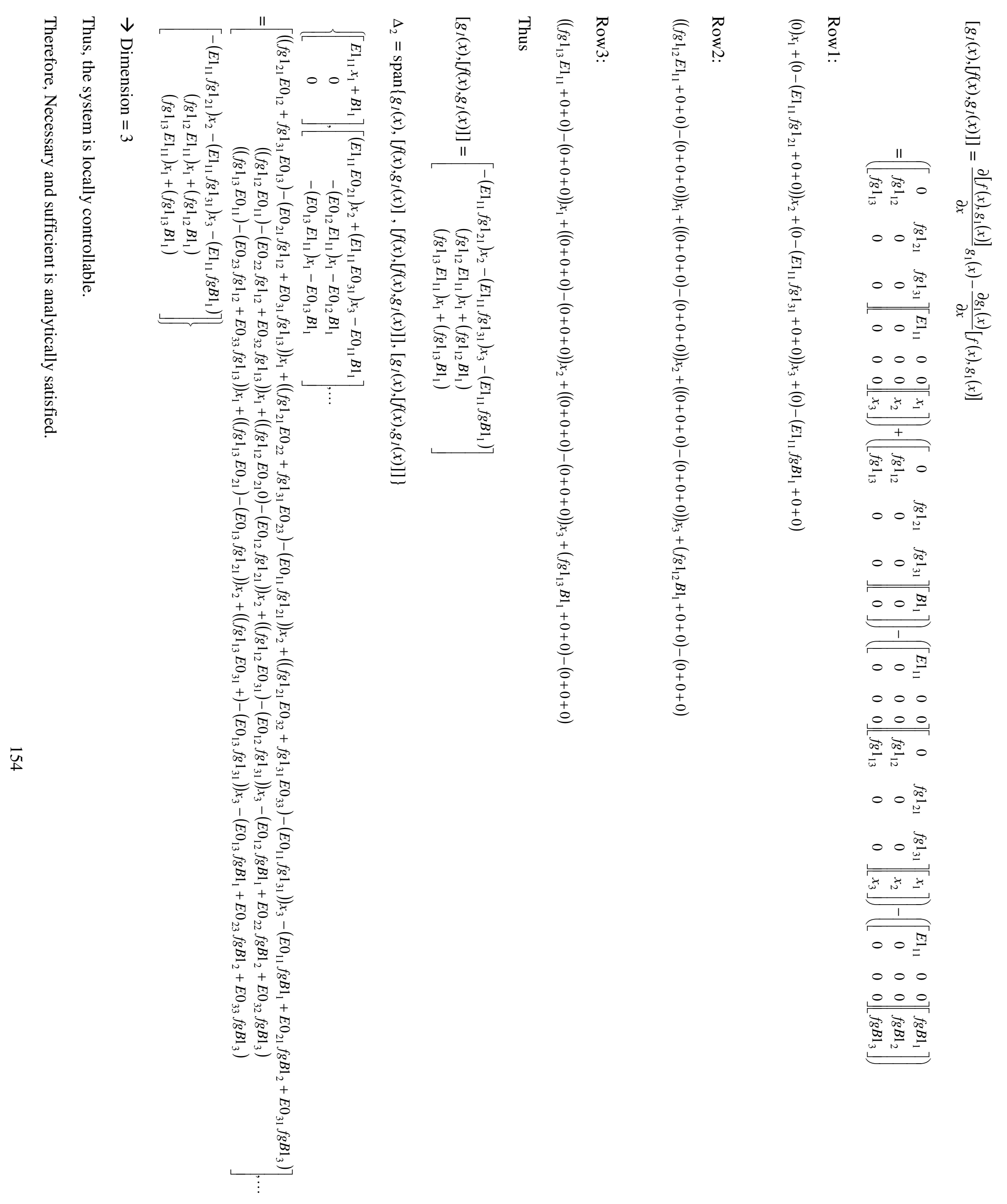




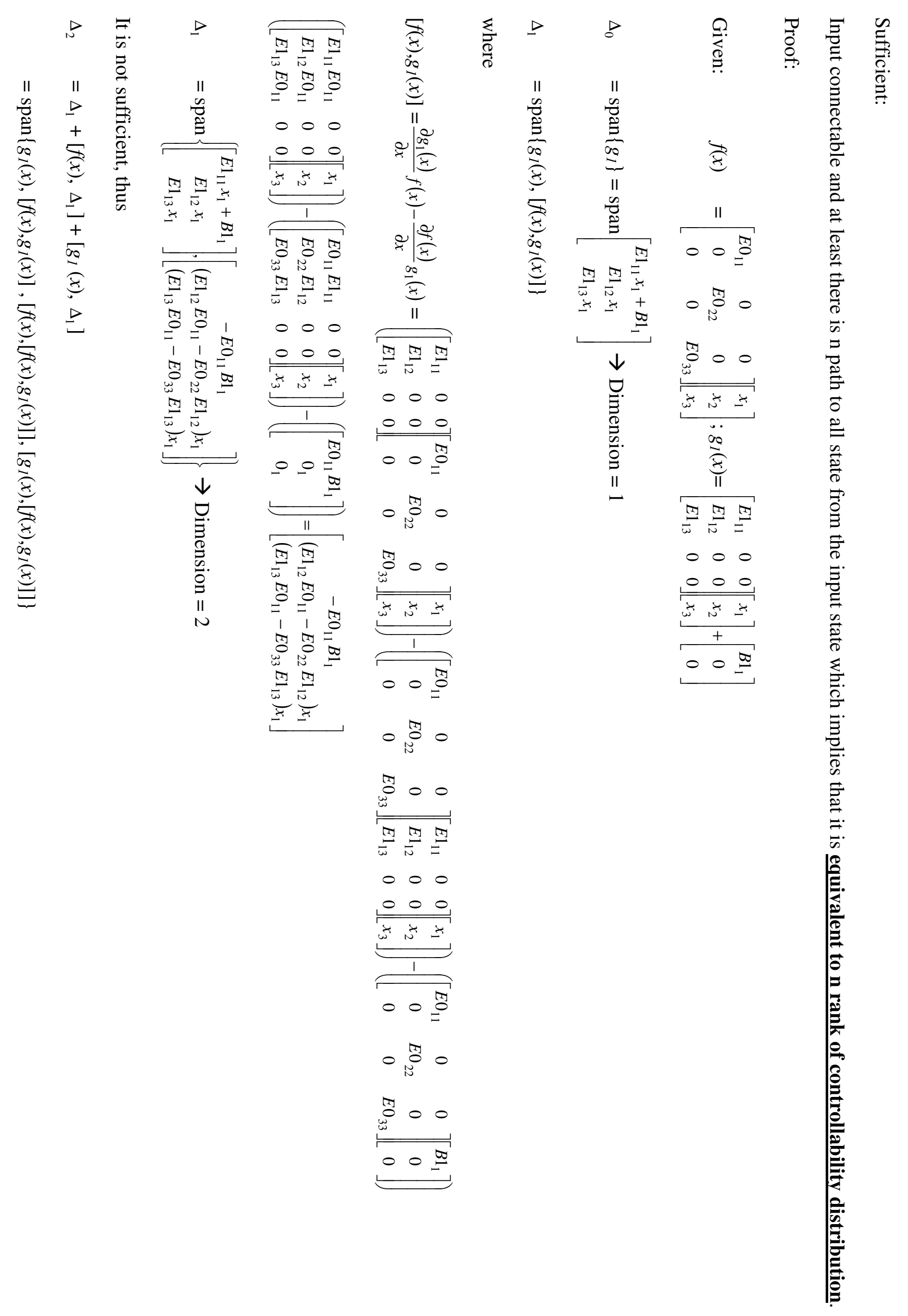




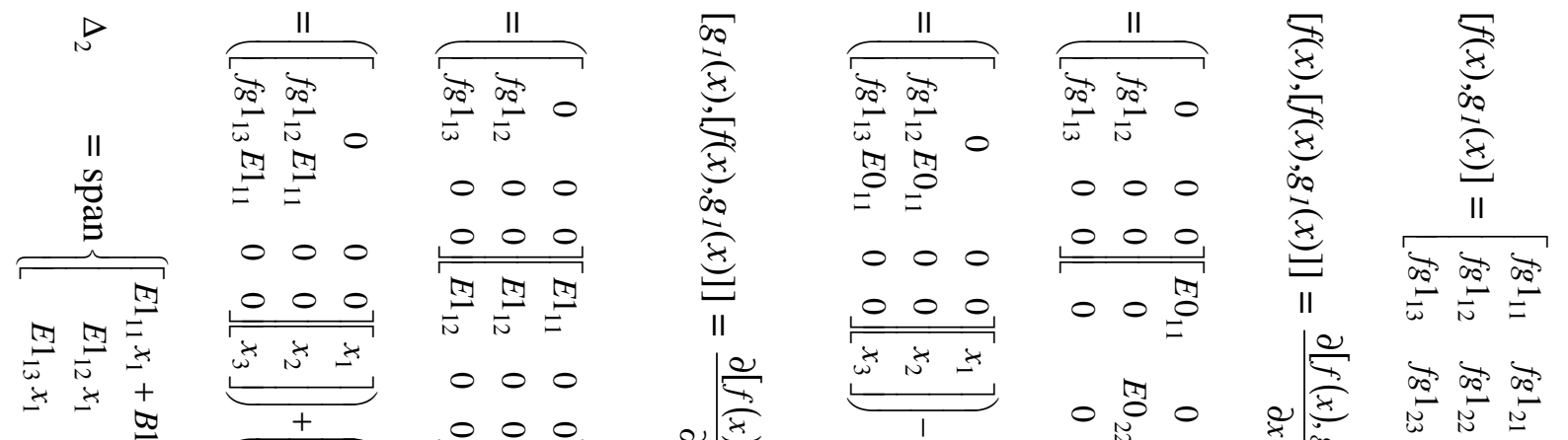
產

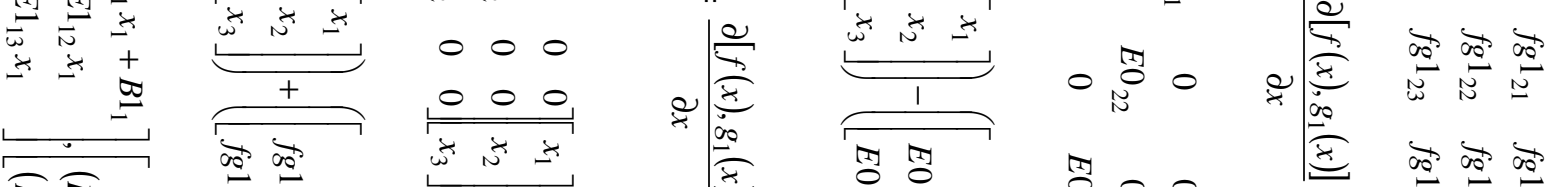

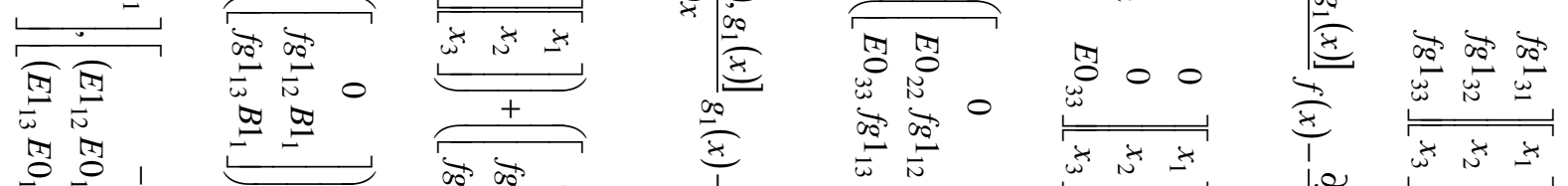

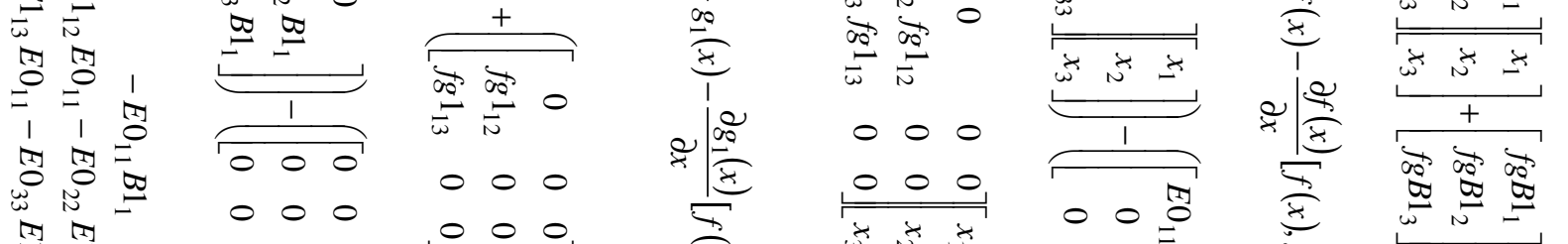

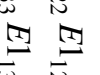

$\stackrel{5}{\square}$

需急

000

000 , 000 i

$\frac{000}{0.000}$

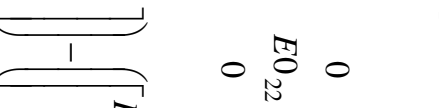

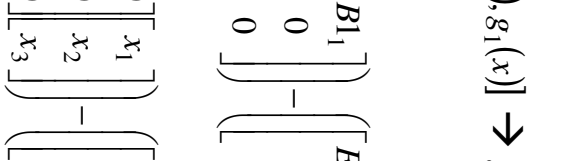

00 엉

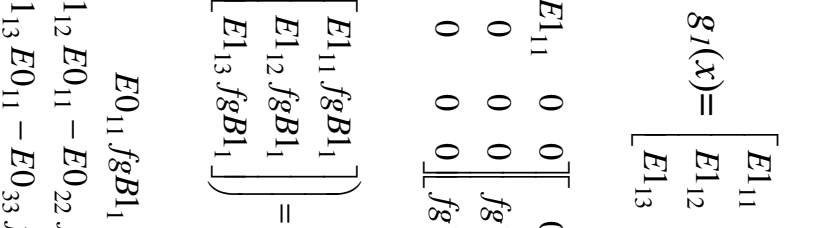

올

$\stackrel{5}{\square}$

两

可高。

$\frac{10}{2}$

家察

일

里

舟

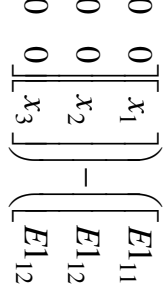

000

00 蛋

영

$\frac{11}{\sqrt{15}}$

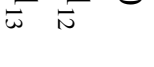

일

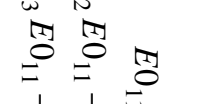

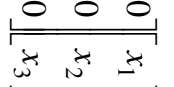

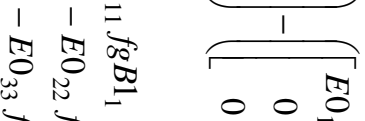

甸画

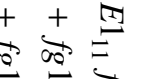

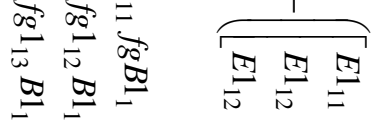

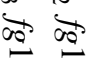

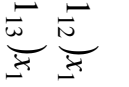

영응

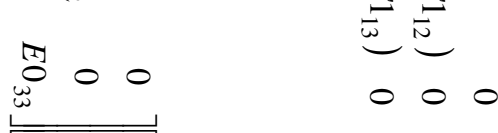

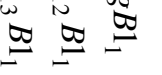

일

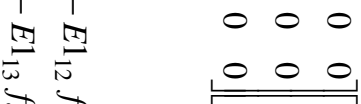

疍总

0 然

蛋兽

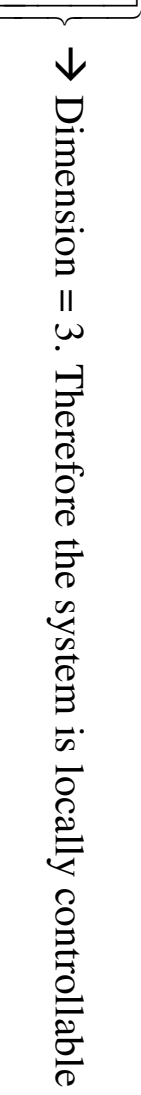

\begin{tabular}{lll}
0 & 0 & 0 \\
\hline & $N$ & $\vdots$
\end{tabular}

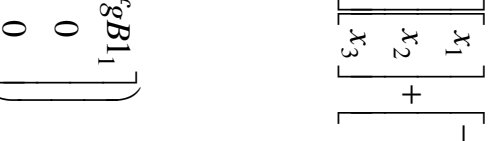

$00 \stackrel{0}{0}$

$\frac{\vec{\omega}}{\frac{m}{\infty}}$

000

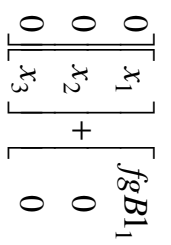



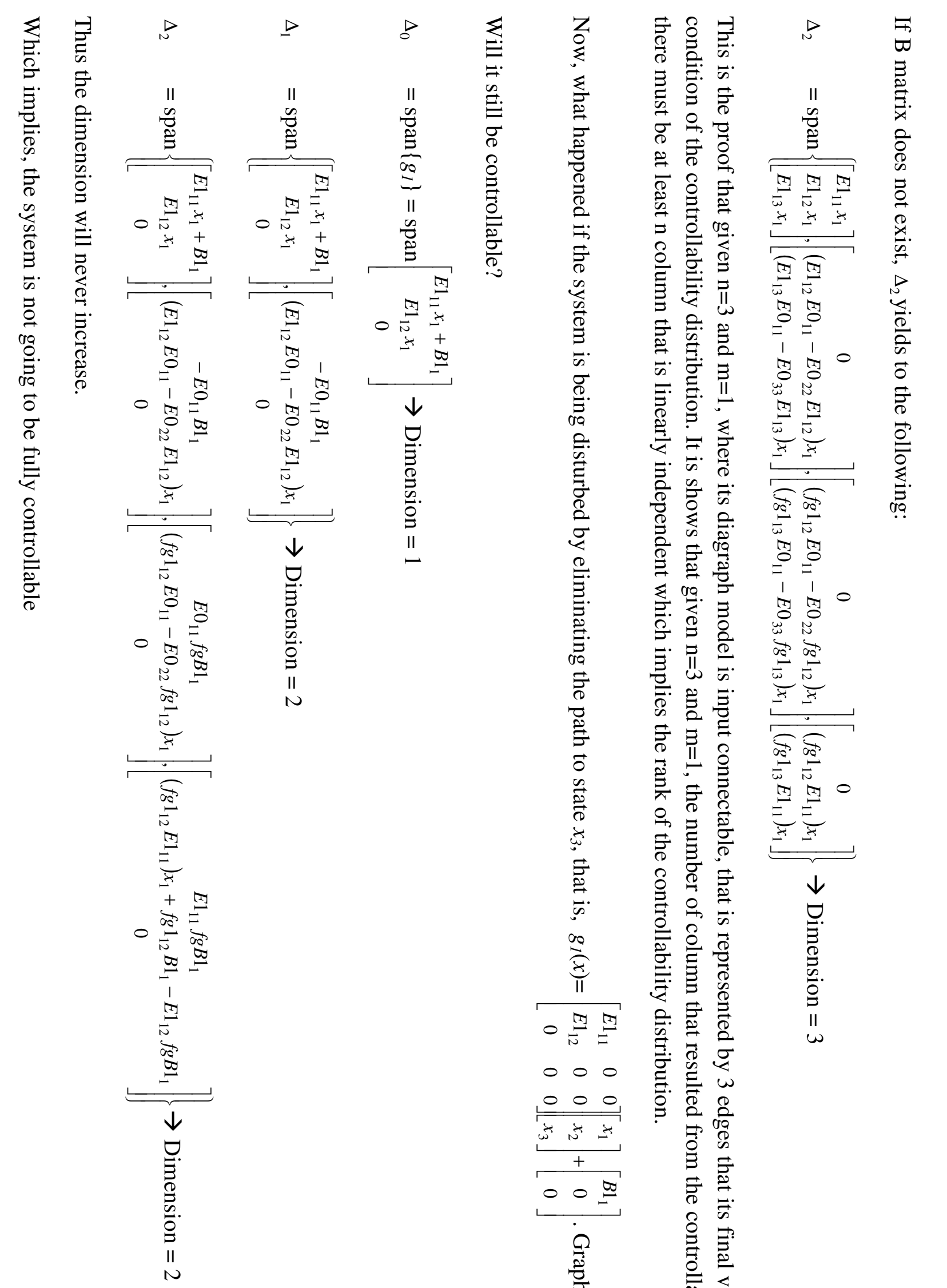

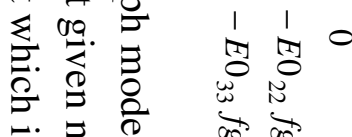

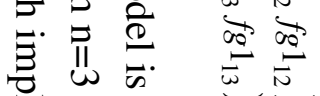

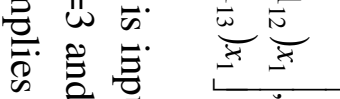

후응

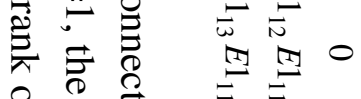

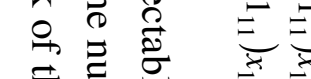

官言葍

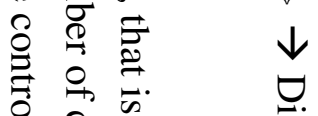

秀

$0 \frac{9}{3} \stackrel{9}{=}$

000

을 휼 है

更

$\circ 00$ 死

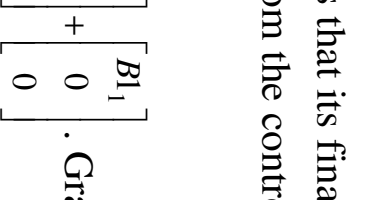

$\overrightarrow{0}$

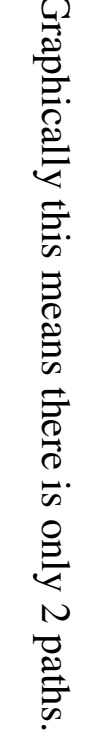

产离

:

官.

․ㅡㄹ

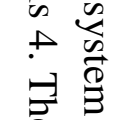

$\begin{array}{ll}1 & \\ 0 & 0 \\ 0 & 0 \\ 0 & 0 \\ 0 & 0 \\ 0\end{array}$

ㅁ. ㄹ. …

$\stackrel{8}{\stackrel{8}{5}}$

高

륭

䒿

产

学

F

完 


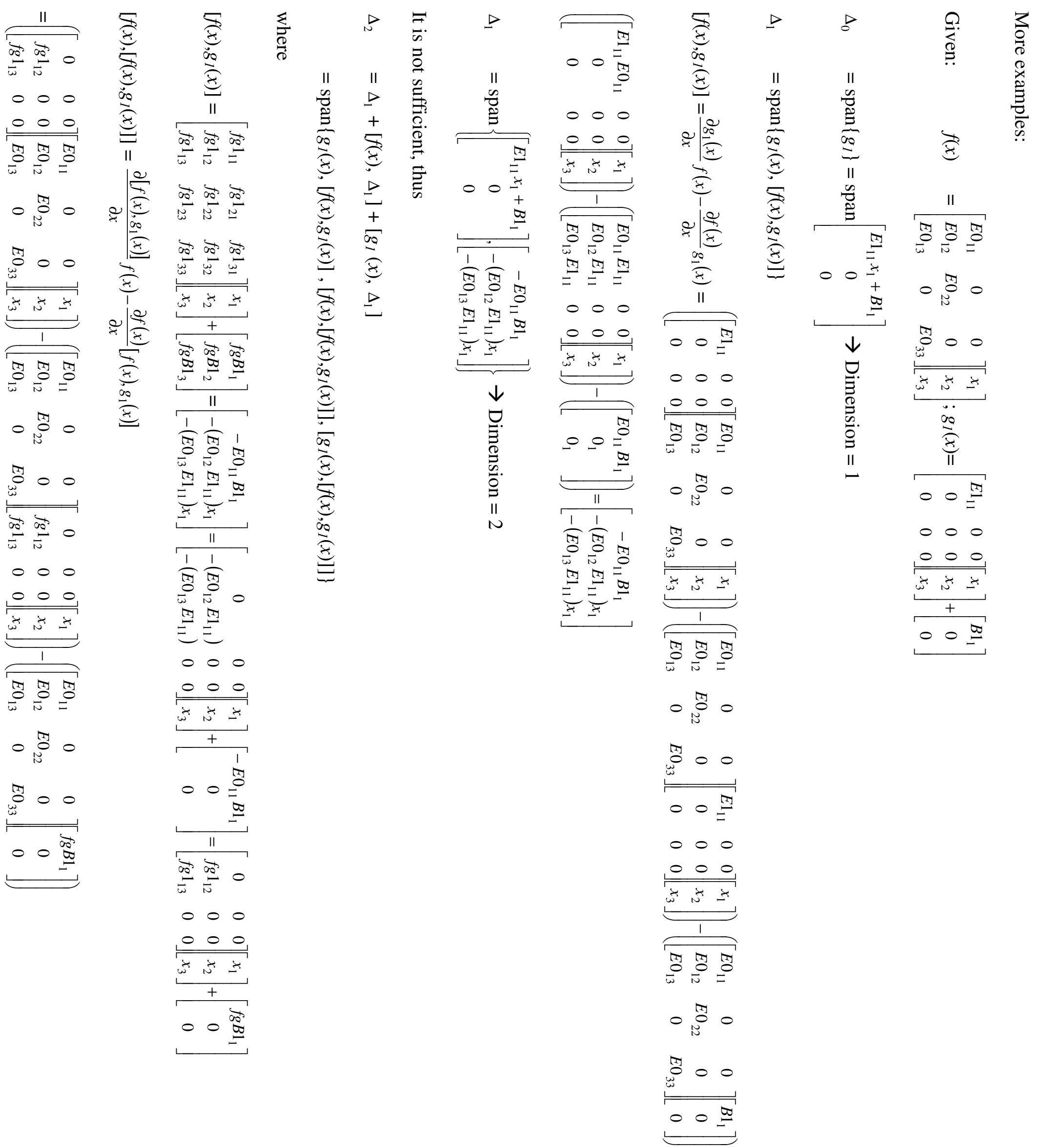




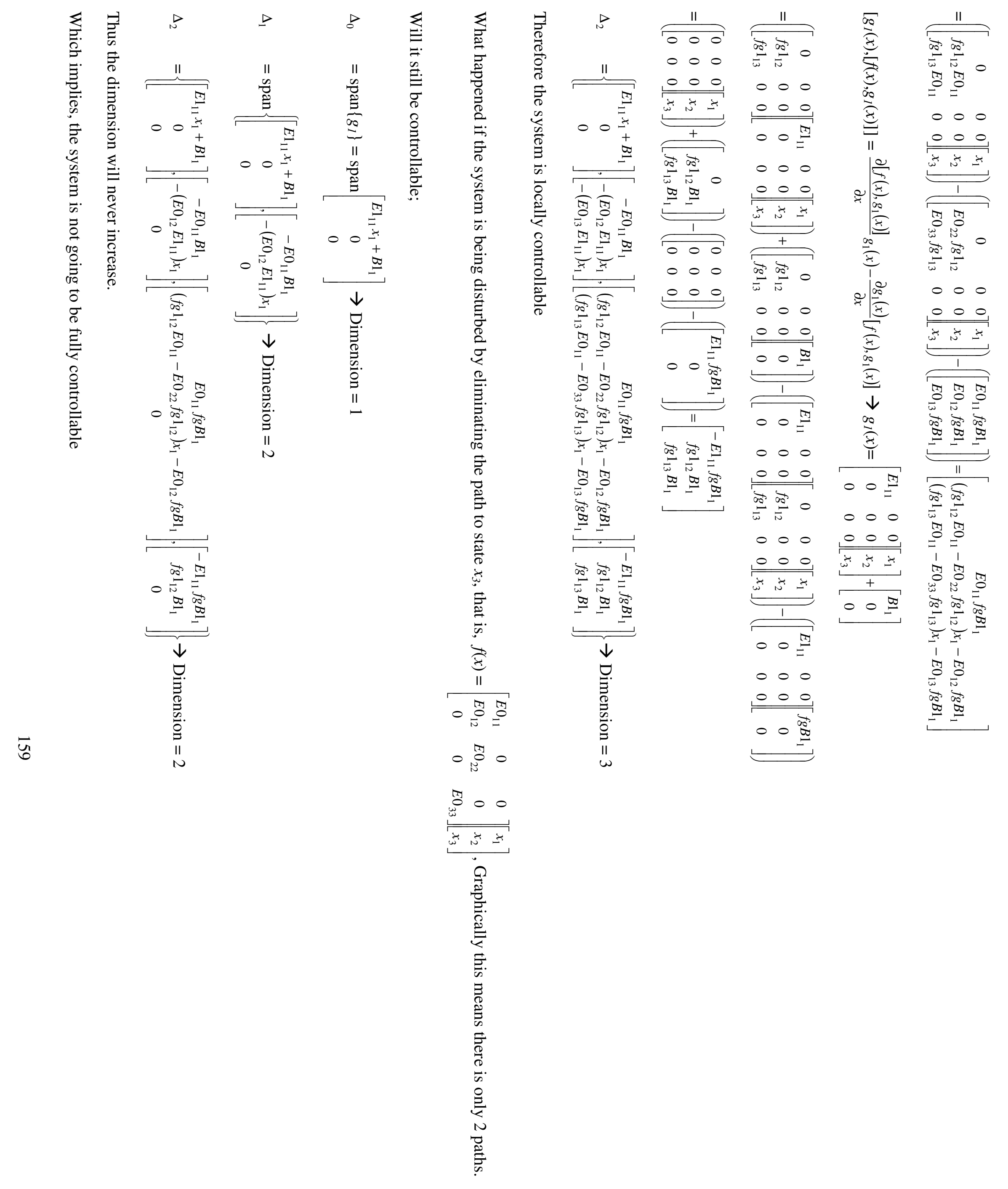



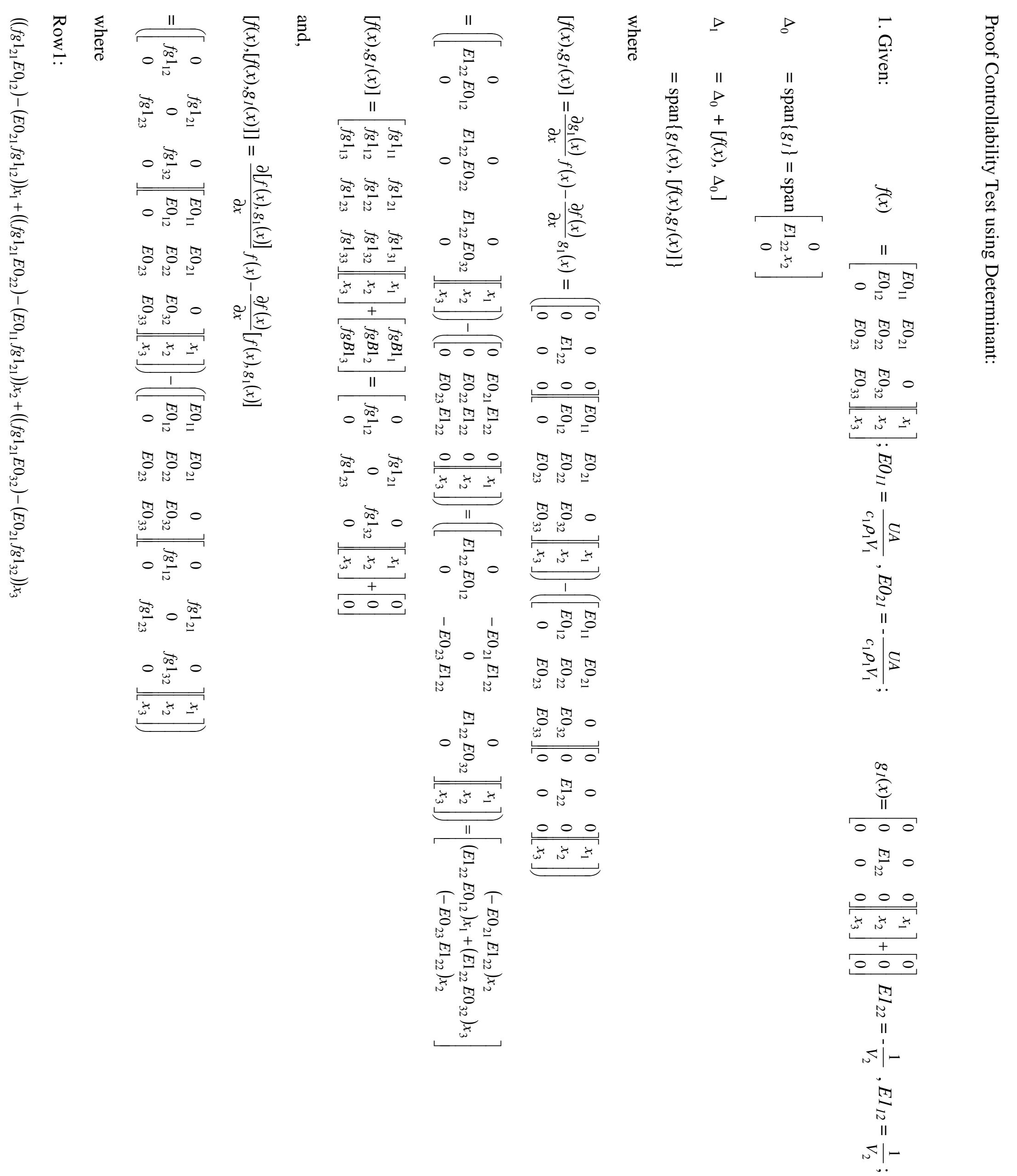


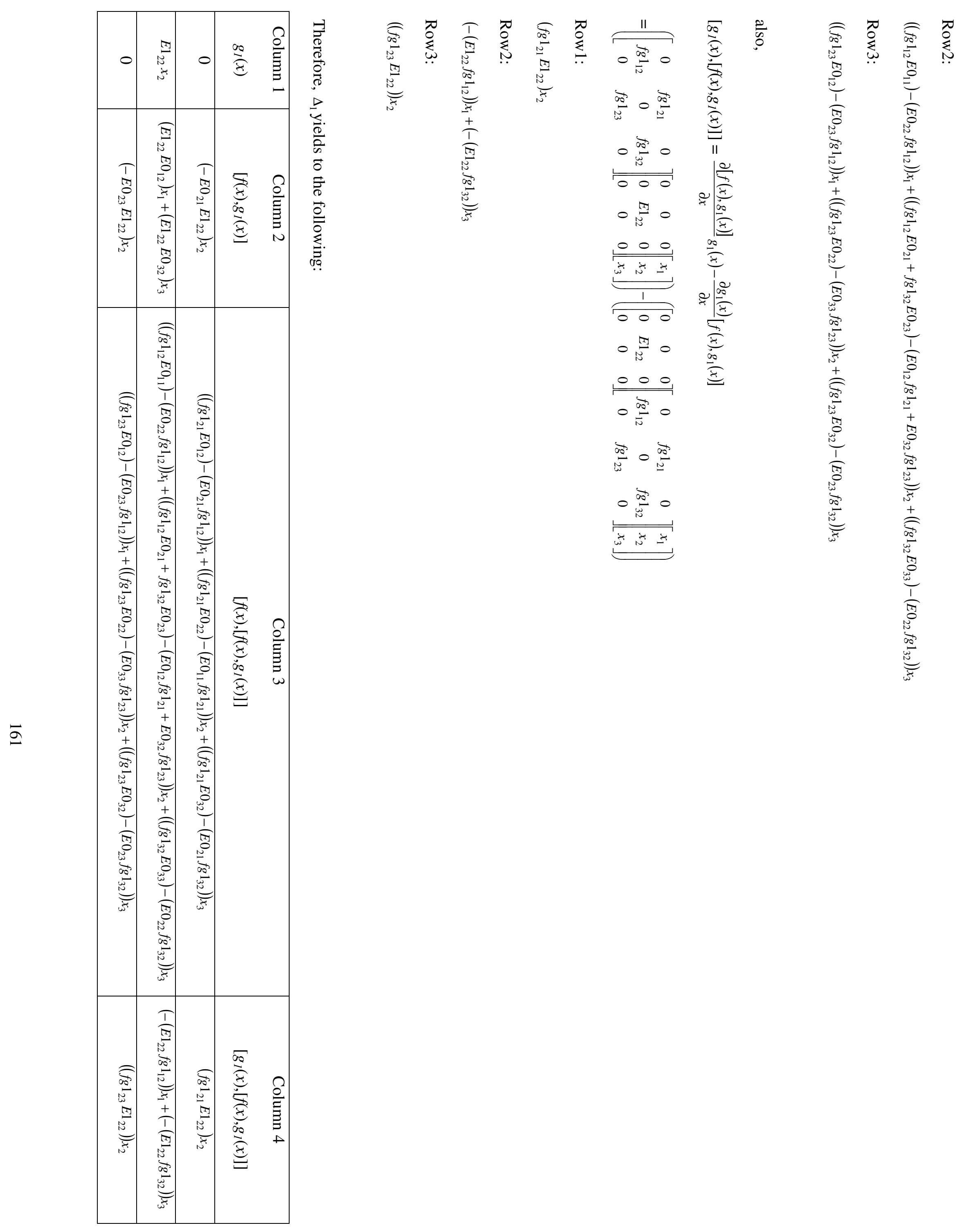




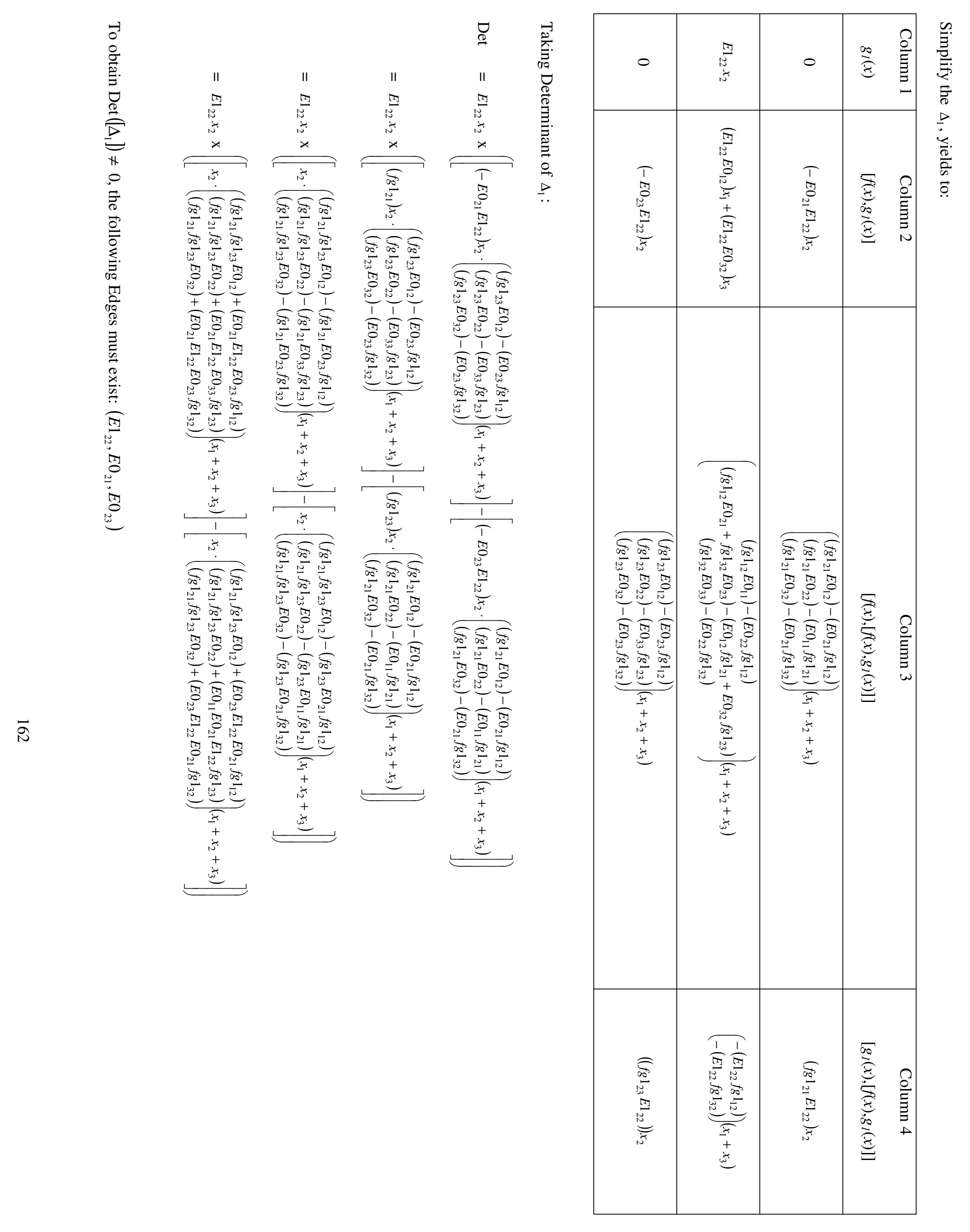


Appendix 4

Observability of Nonlinear System: A proof 


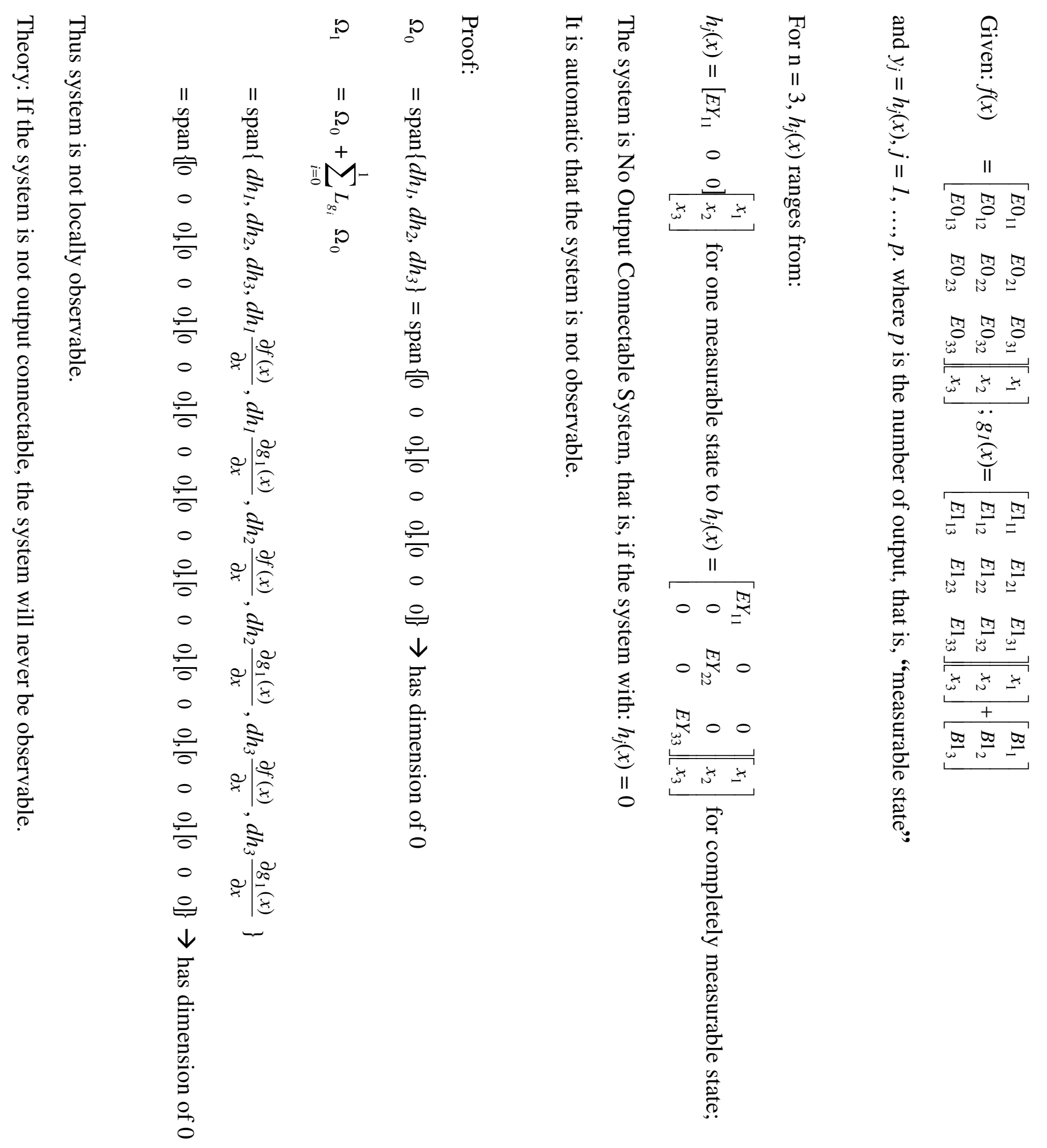




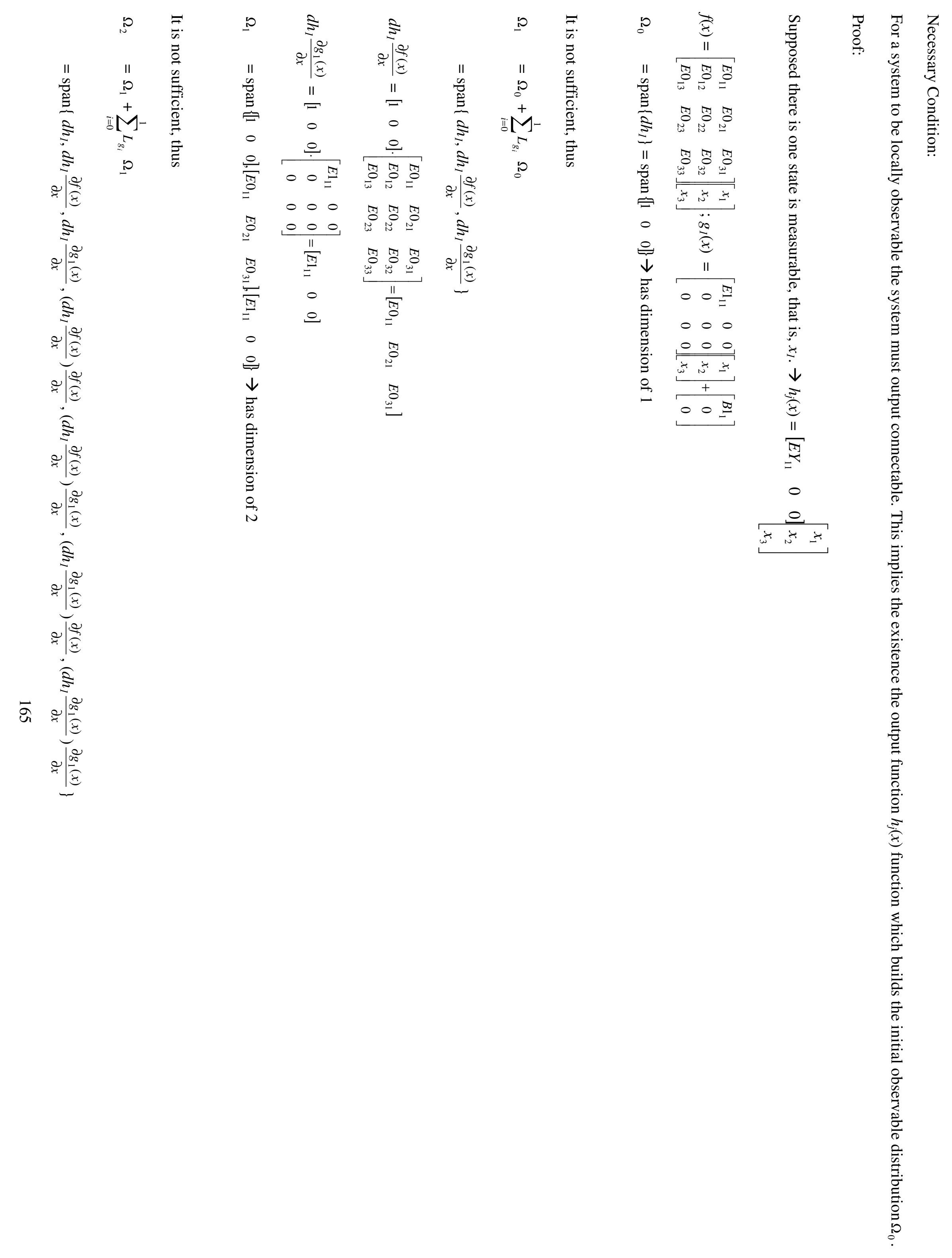



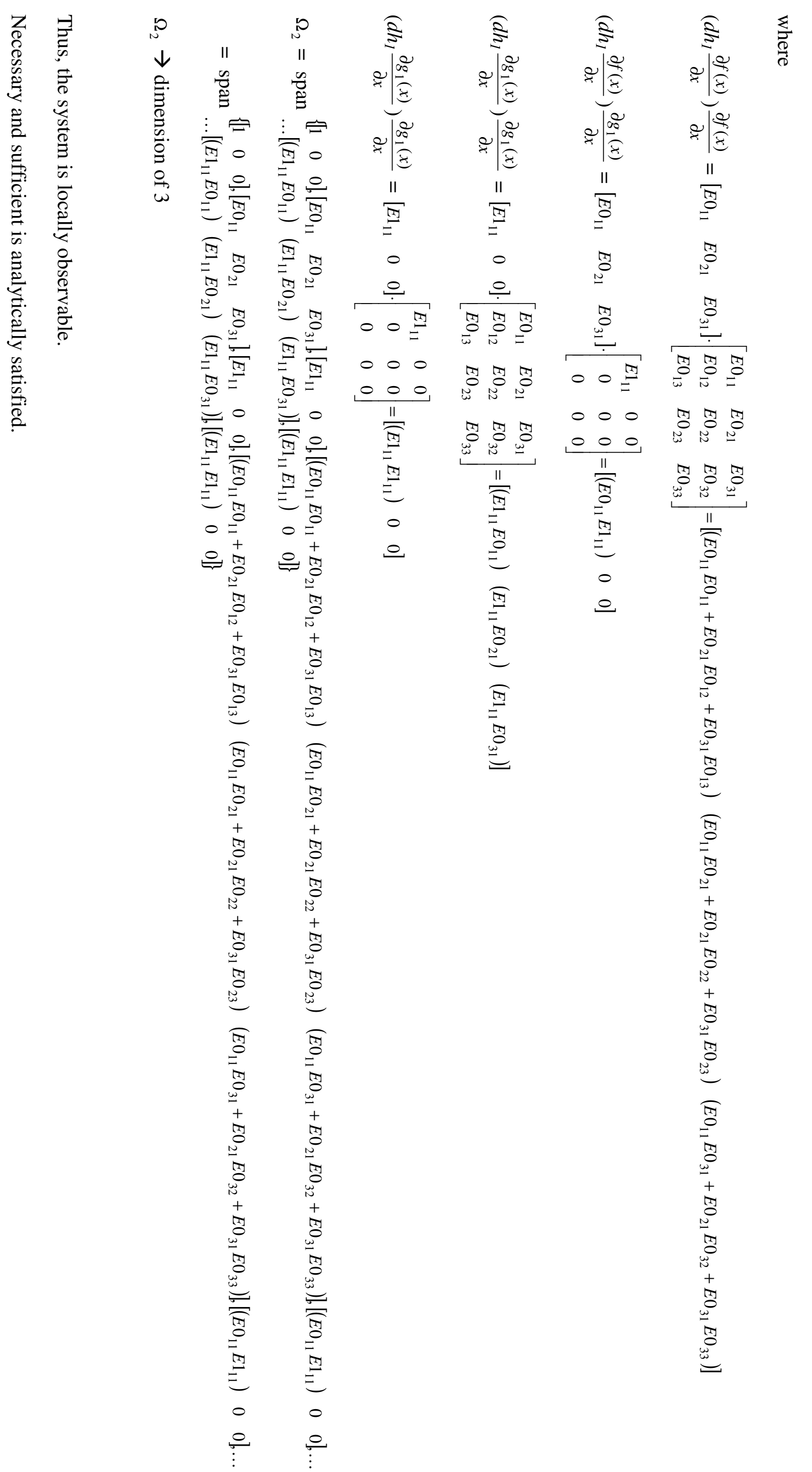

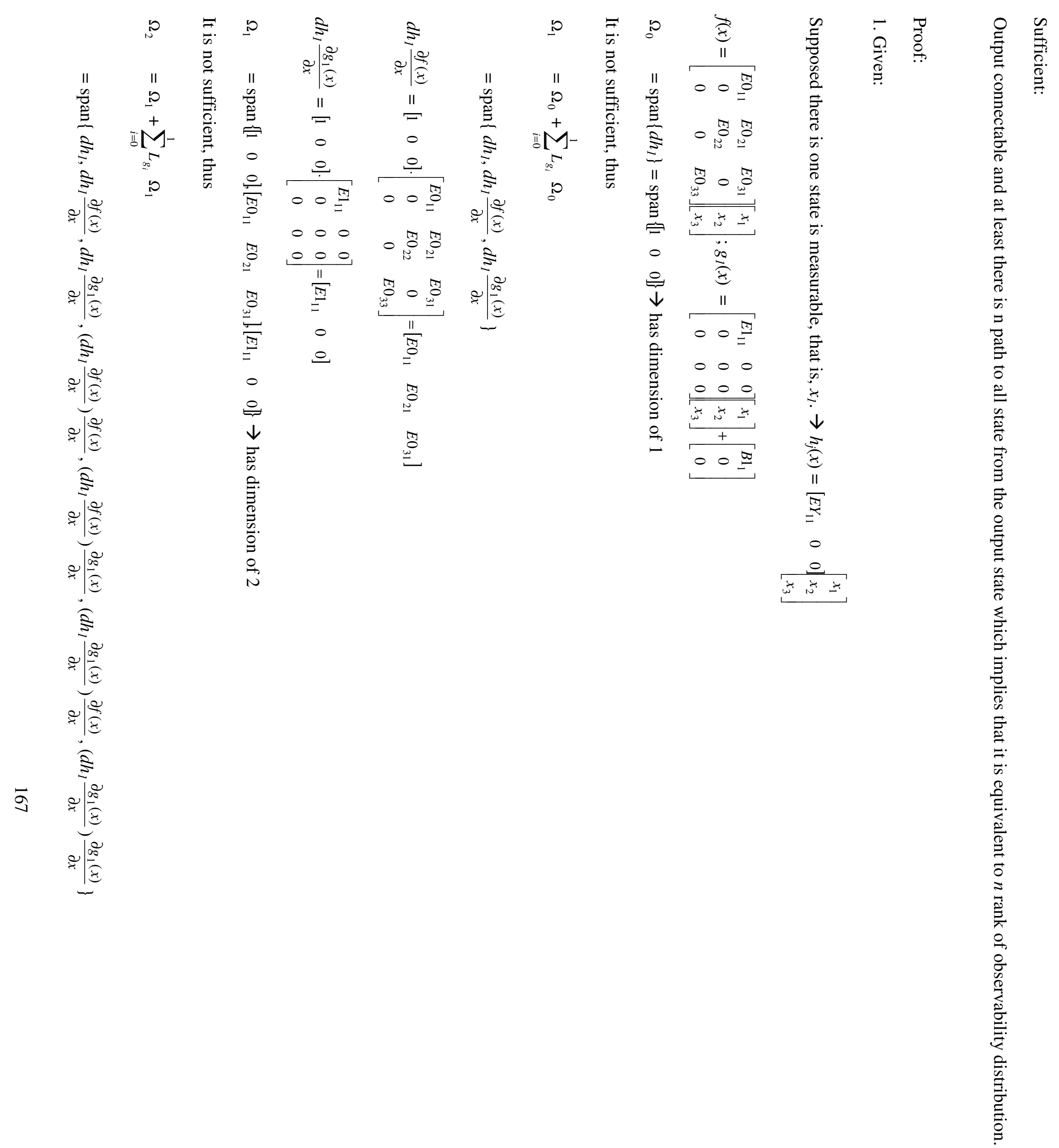


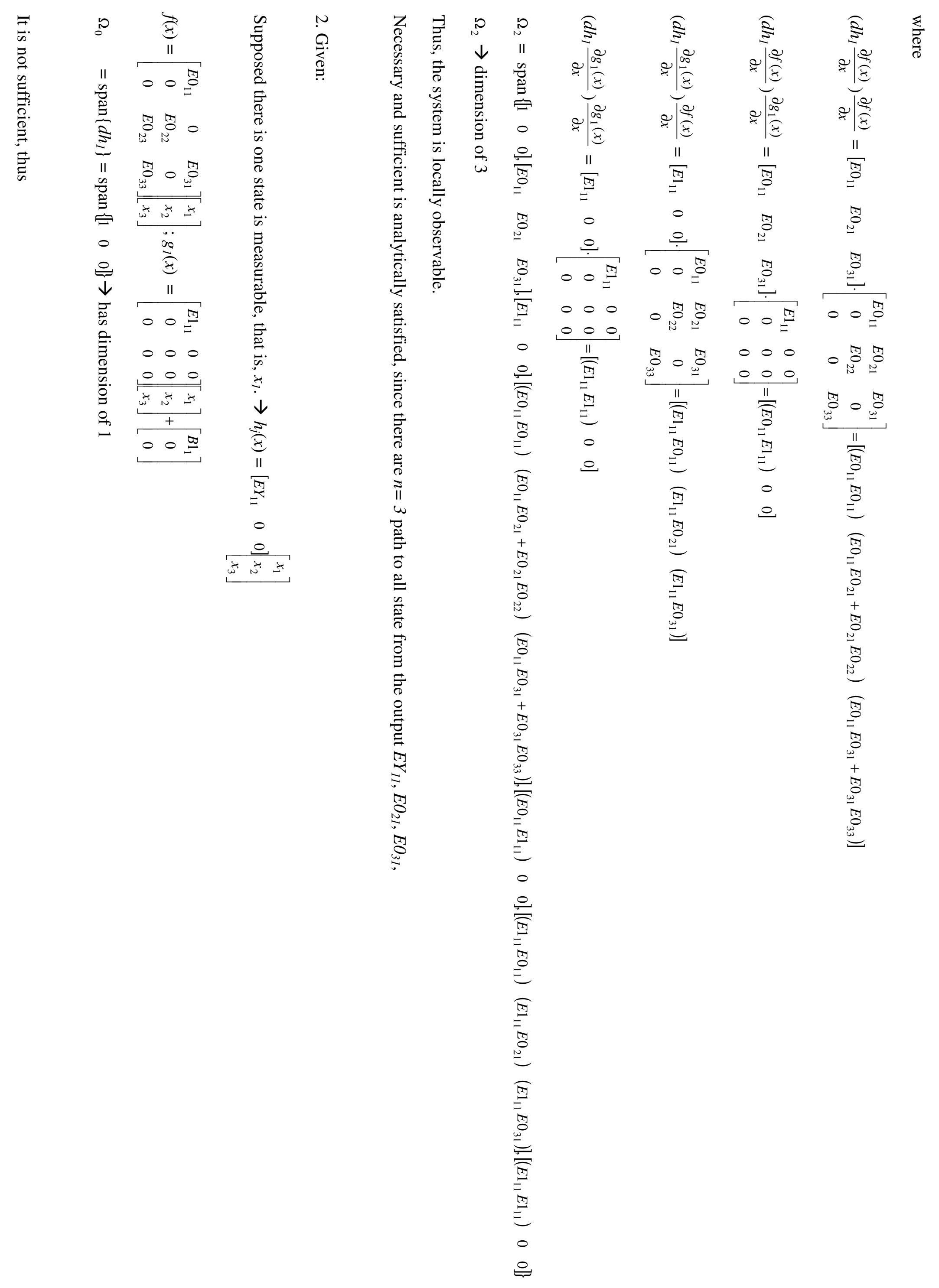




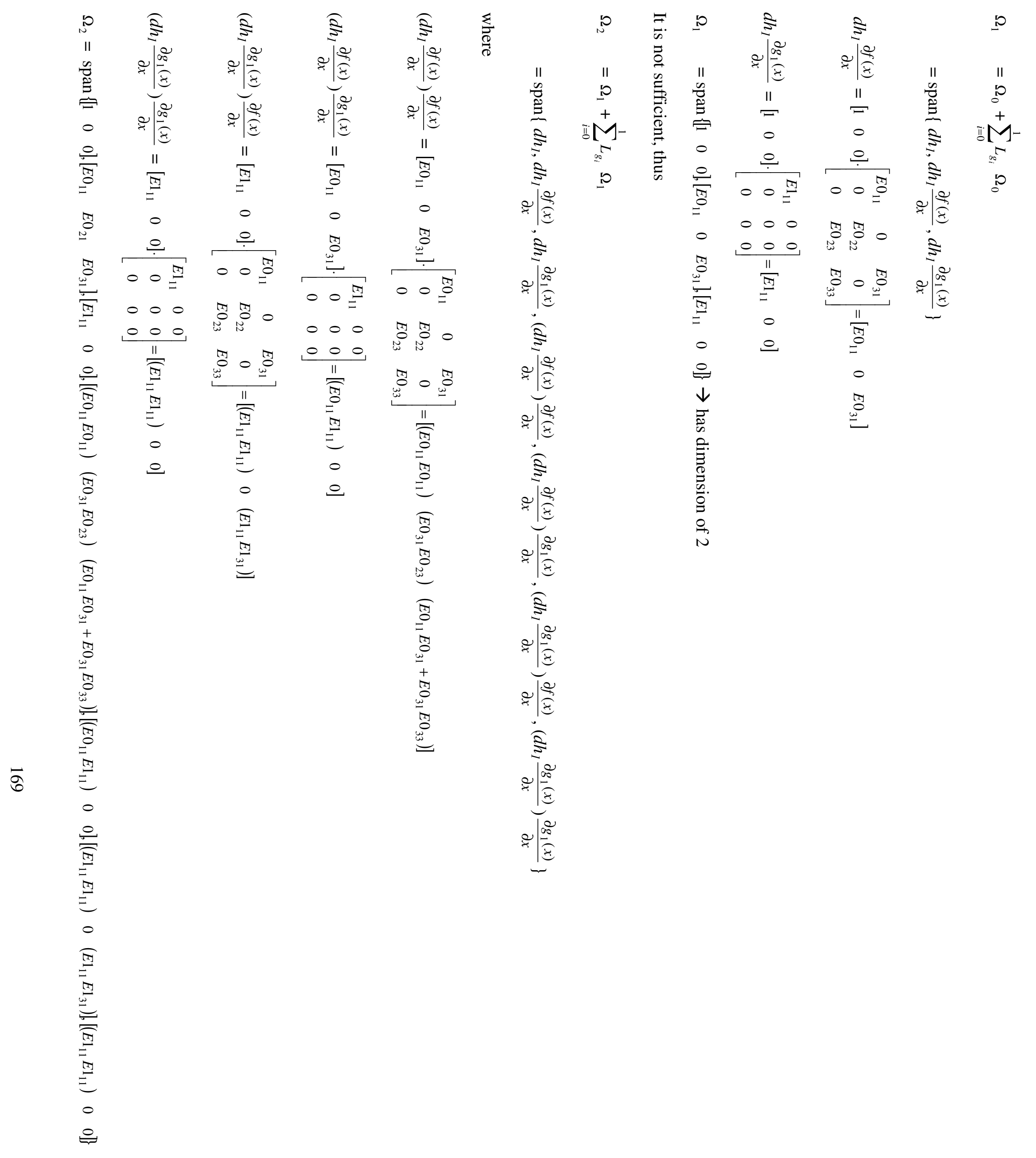




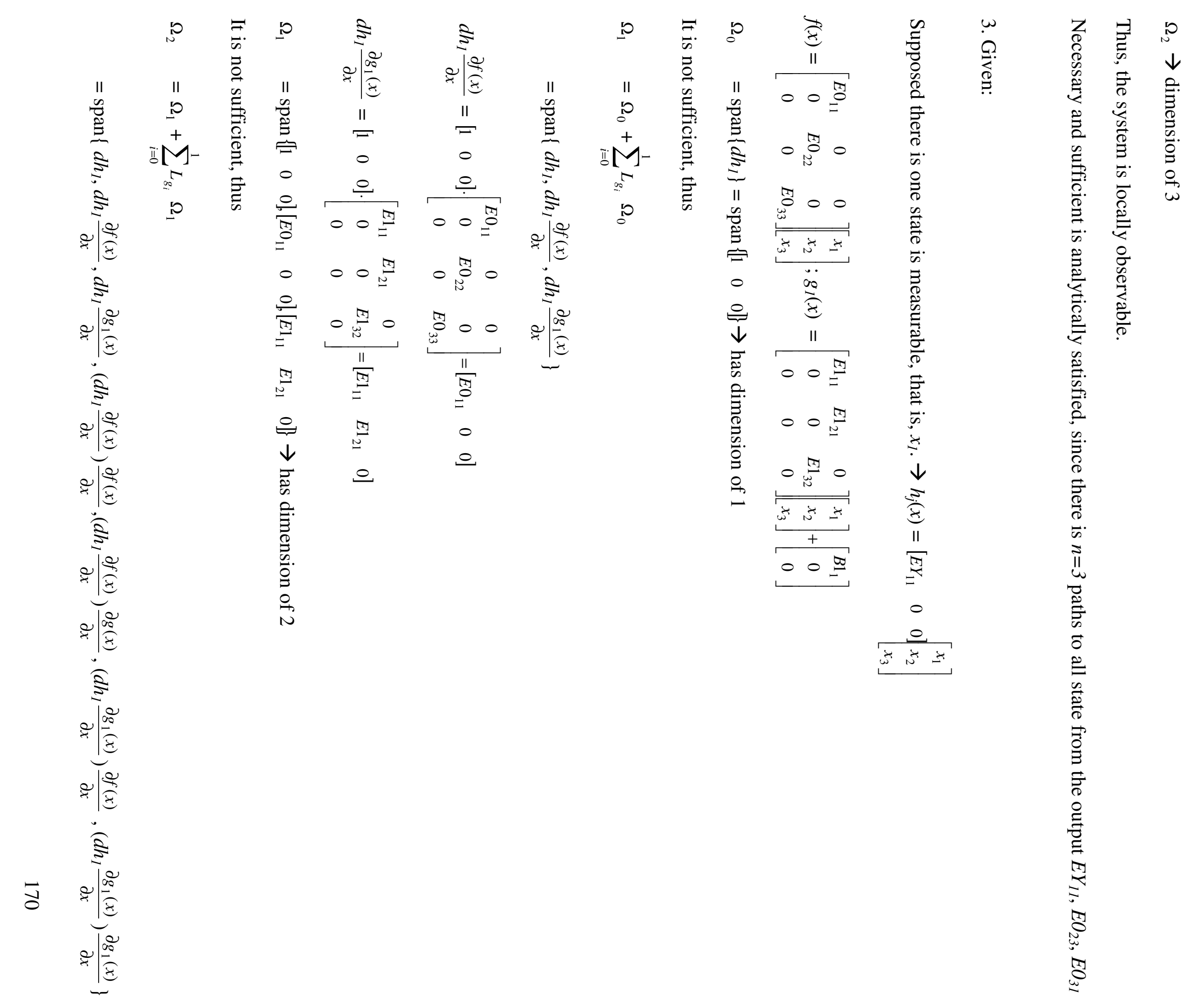



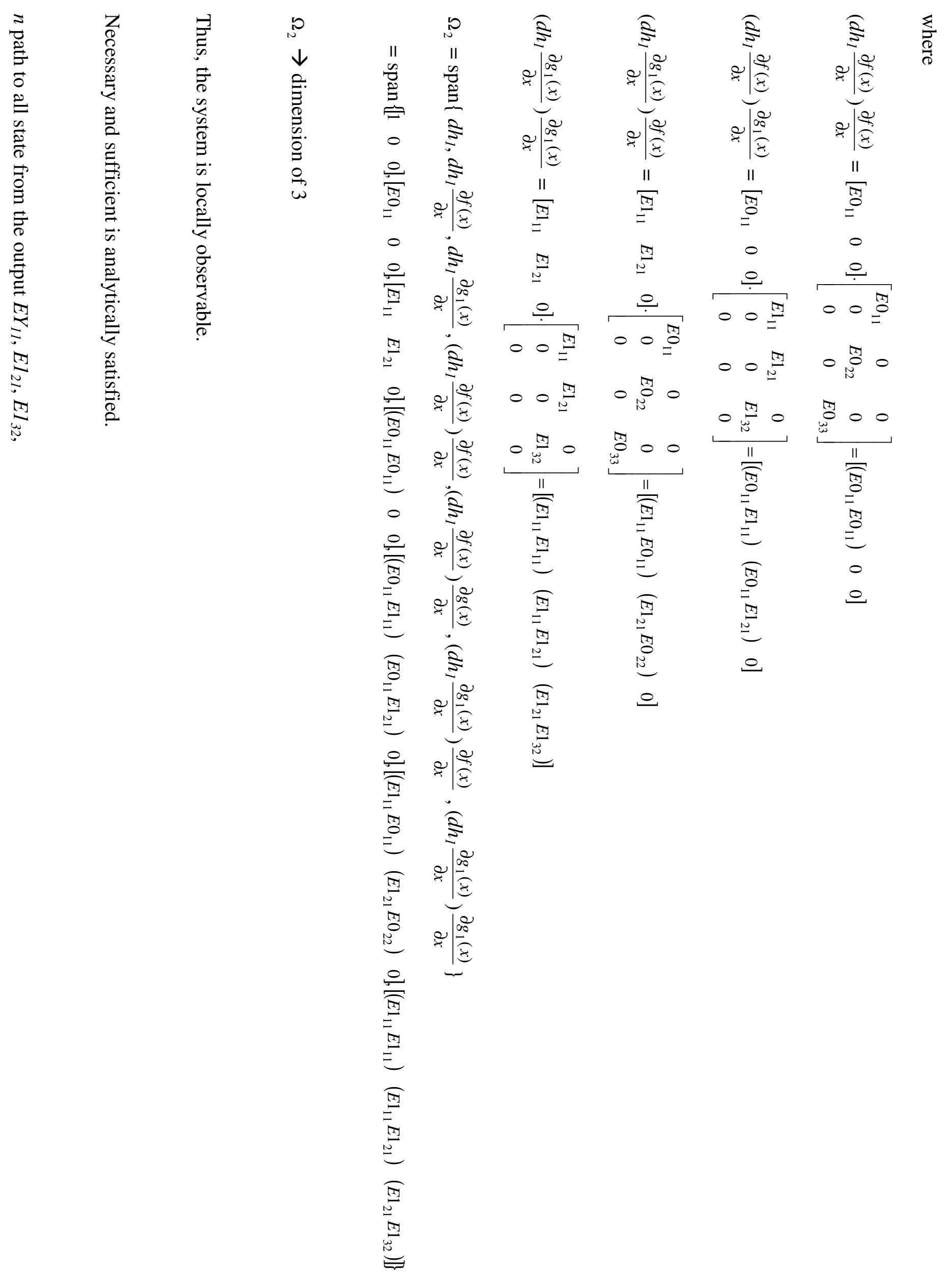


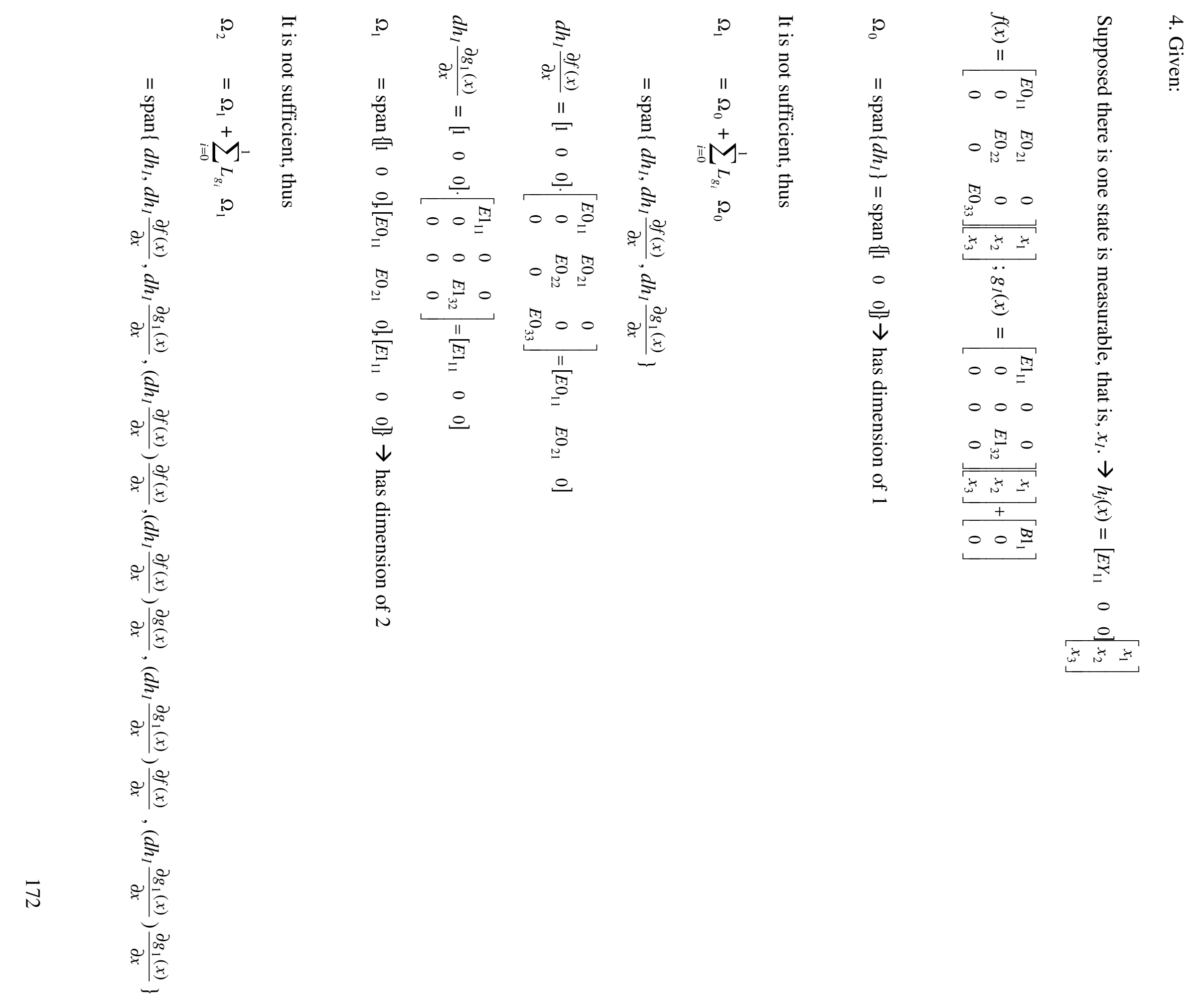



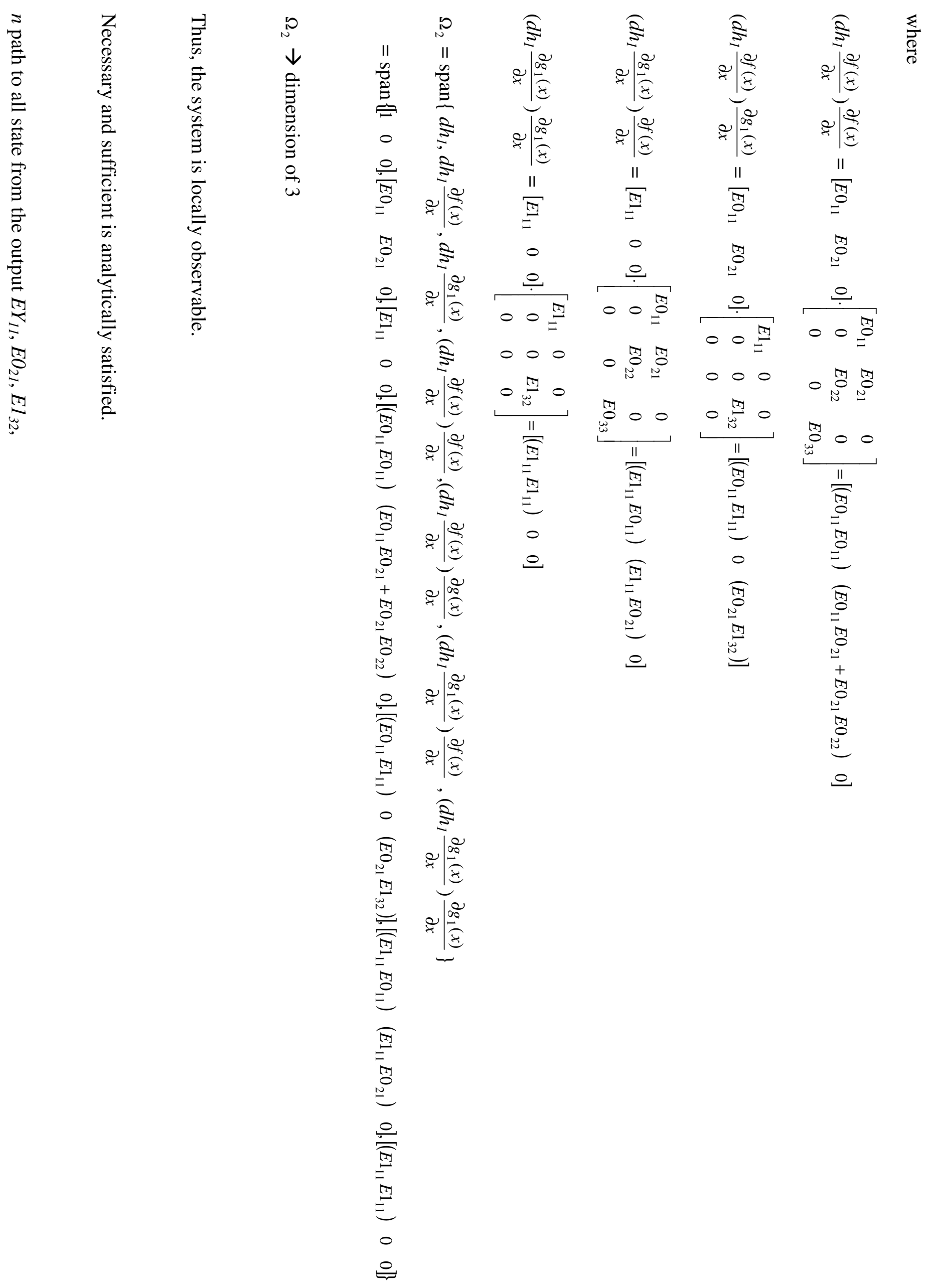


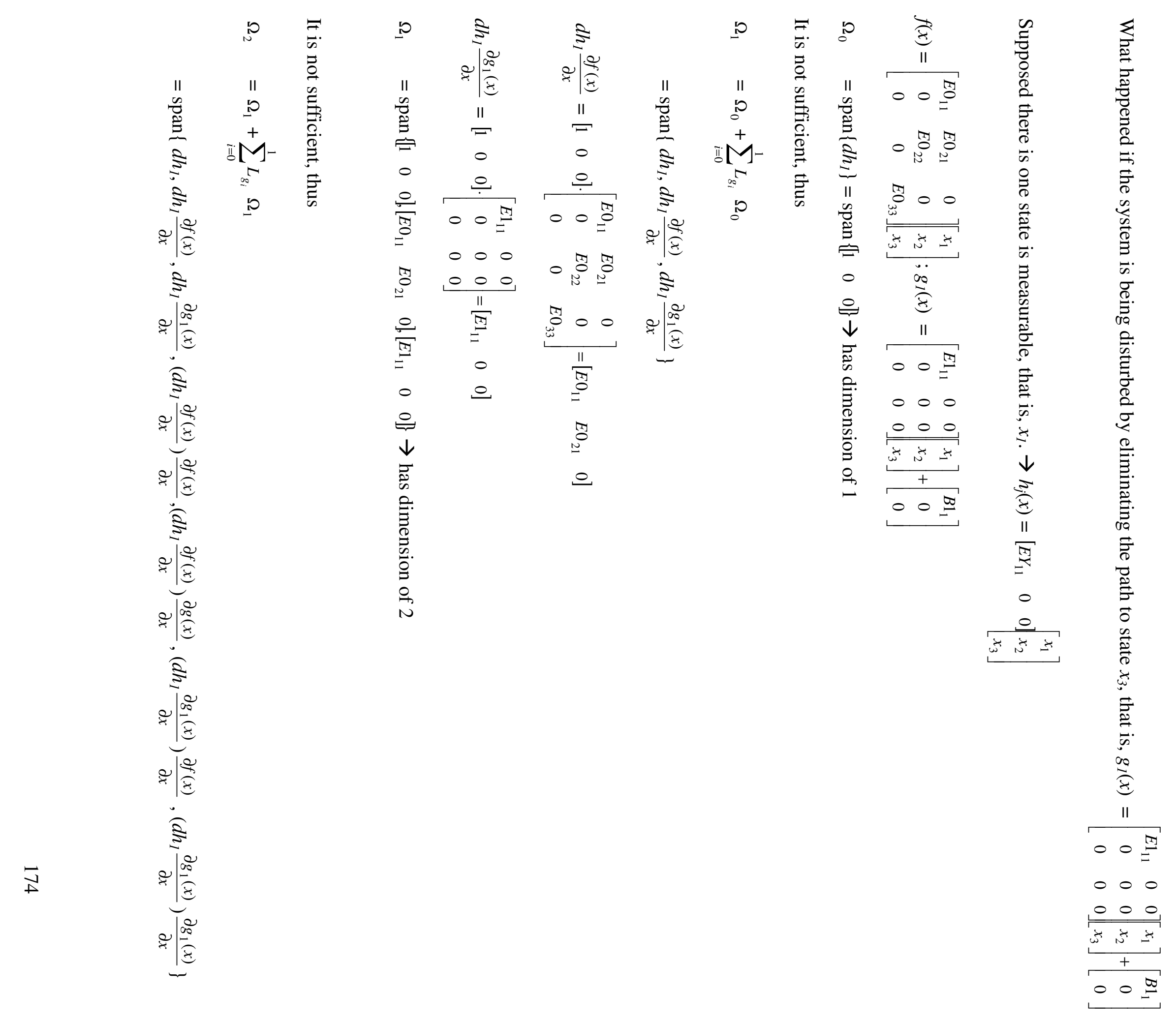




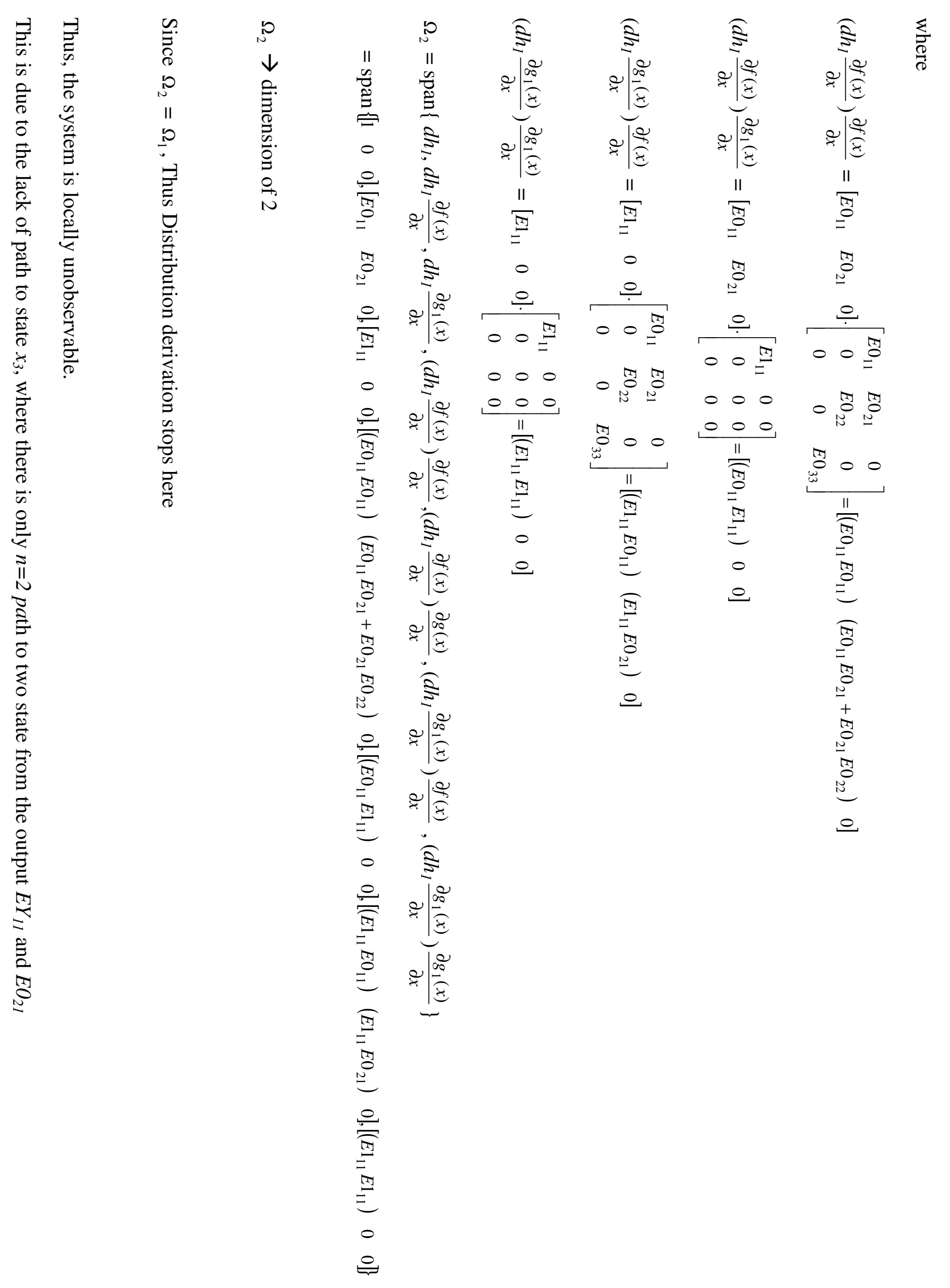




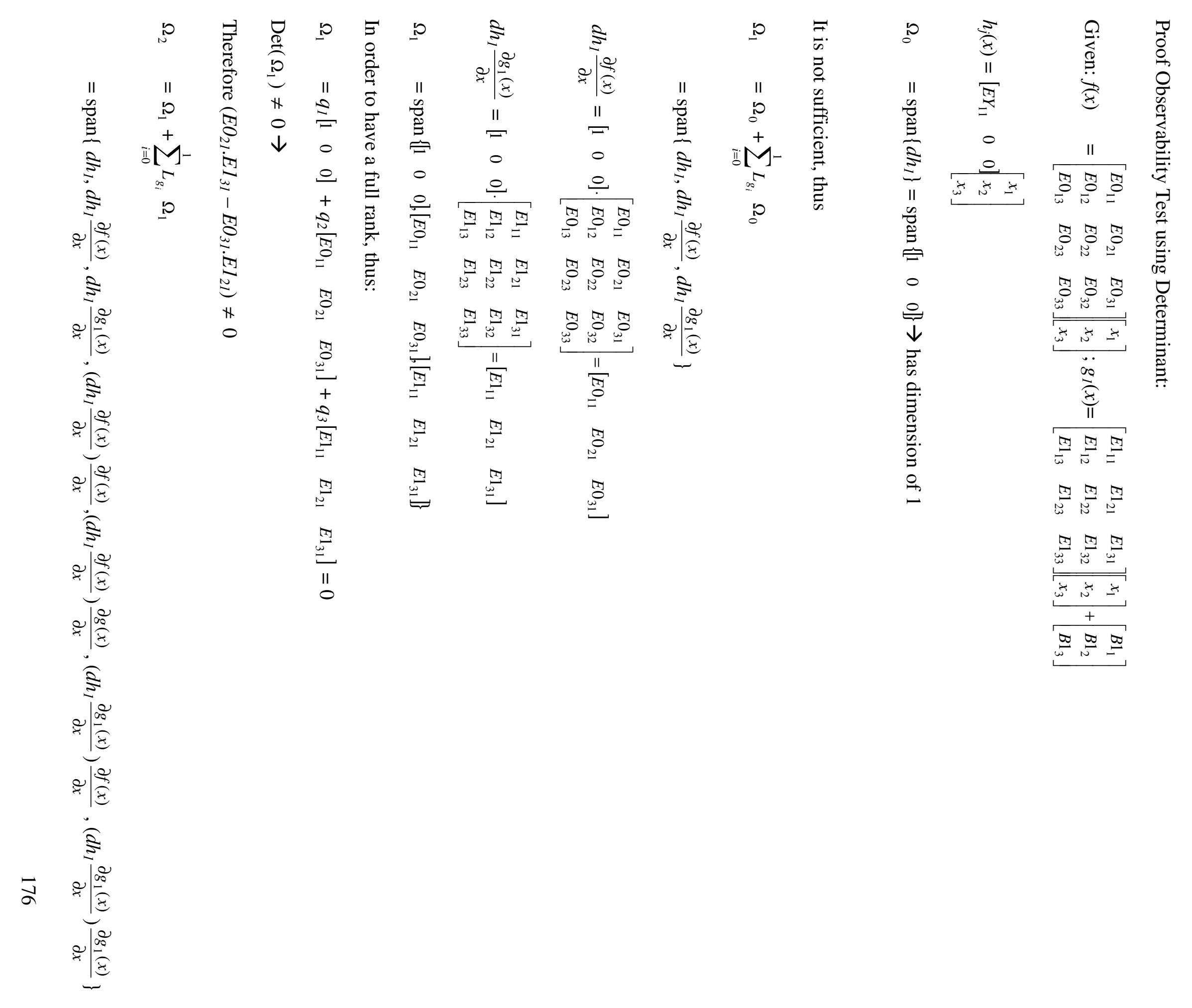




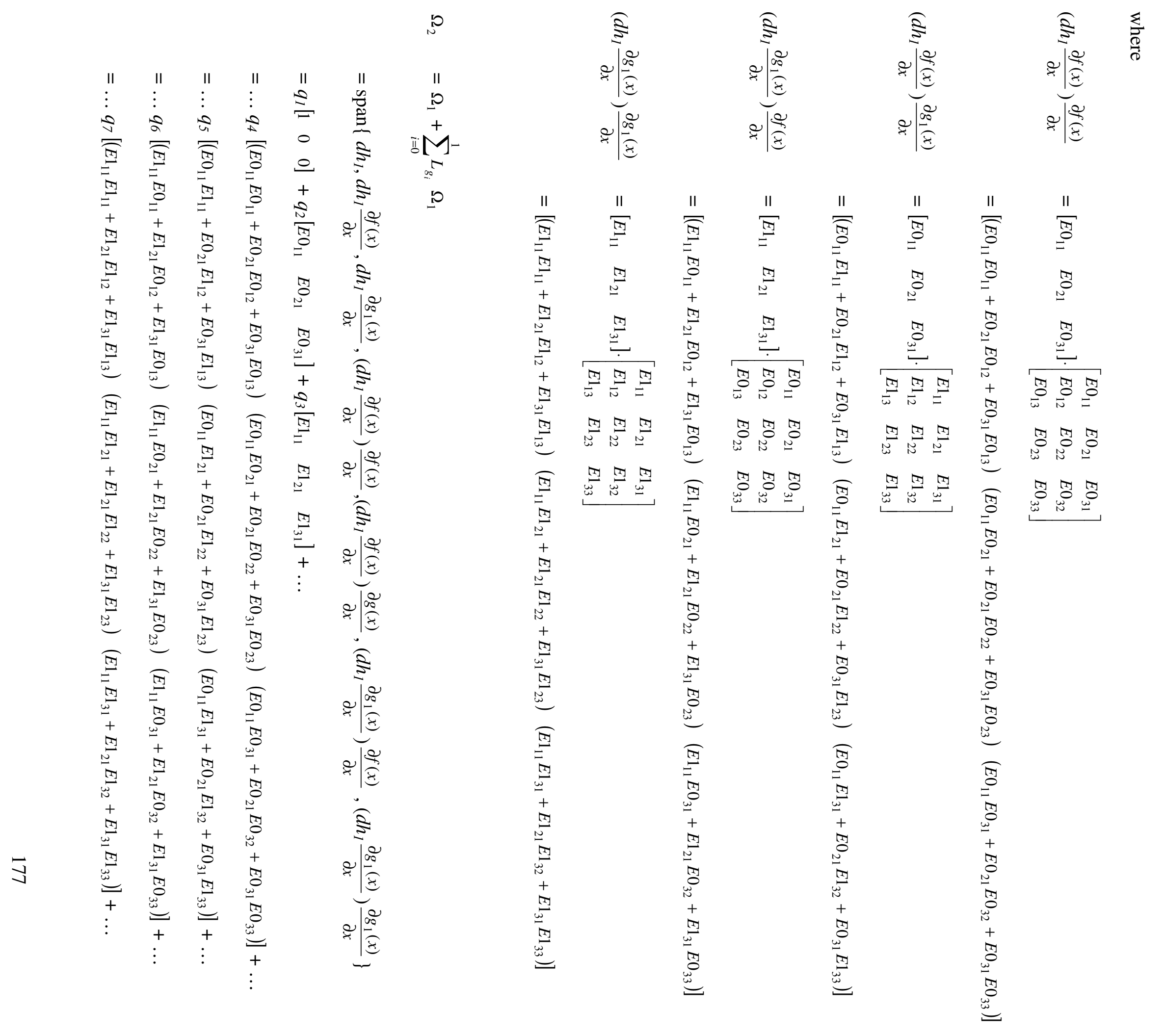




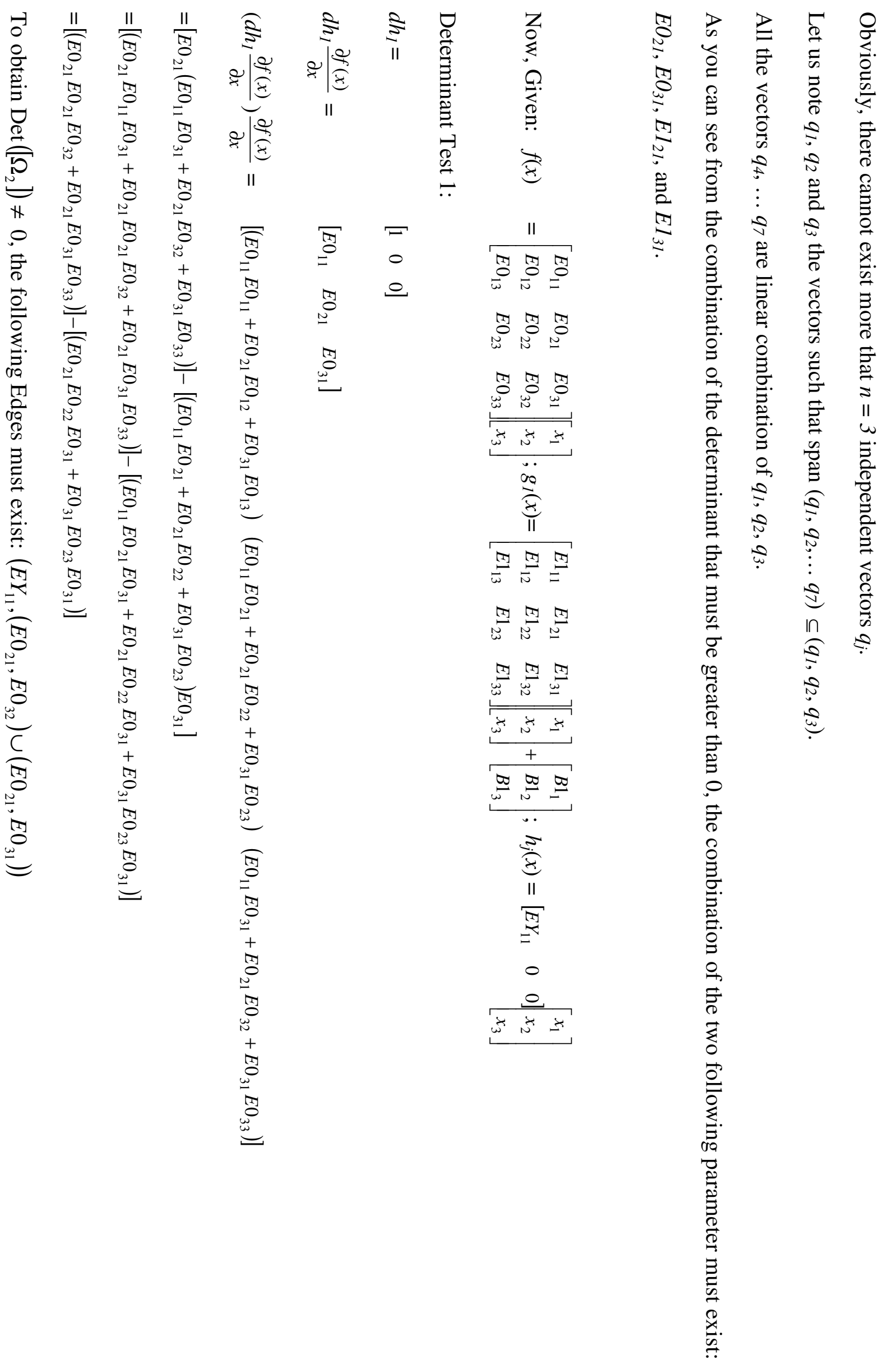




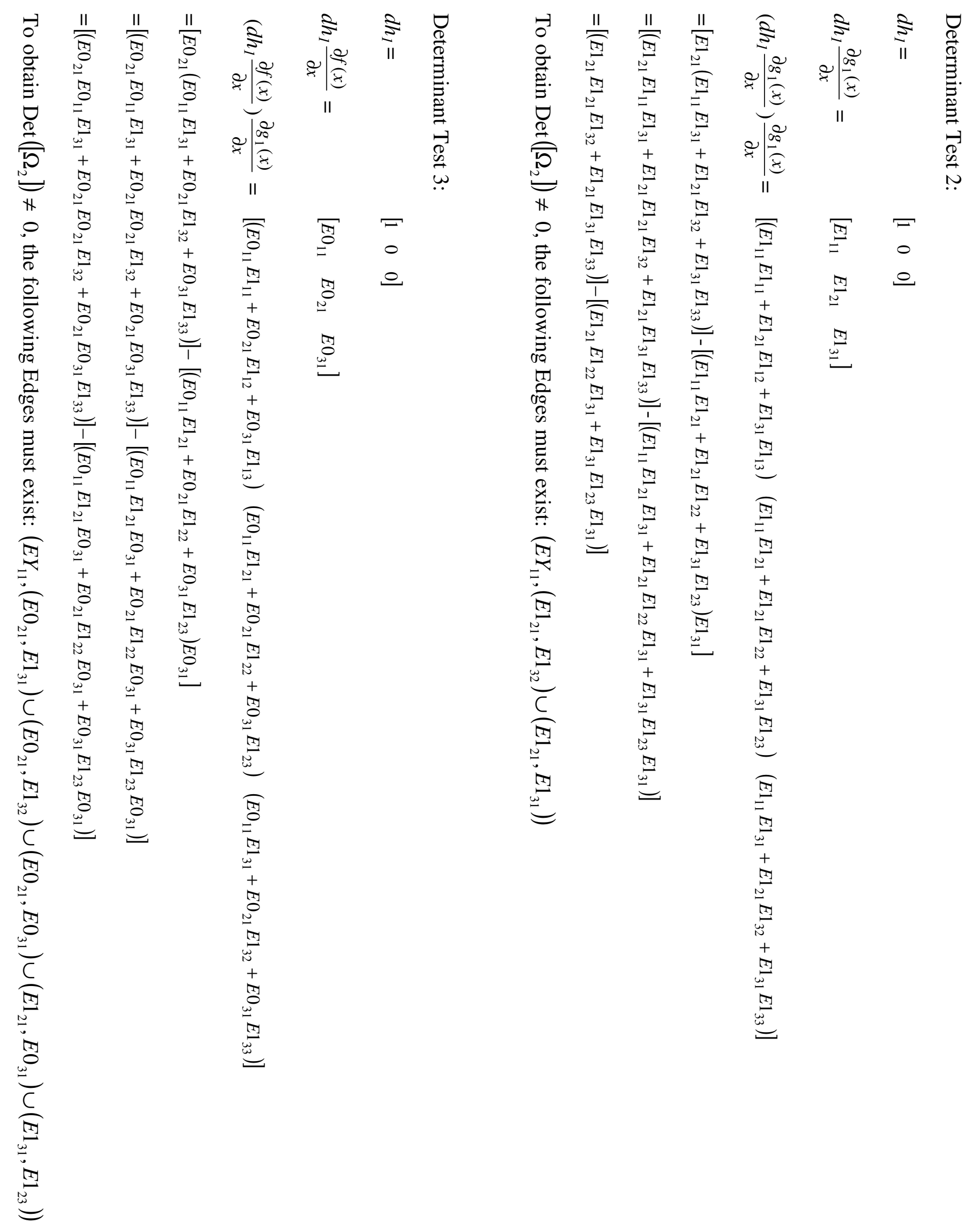




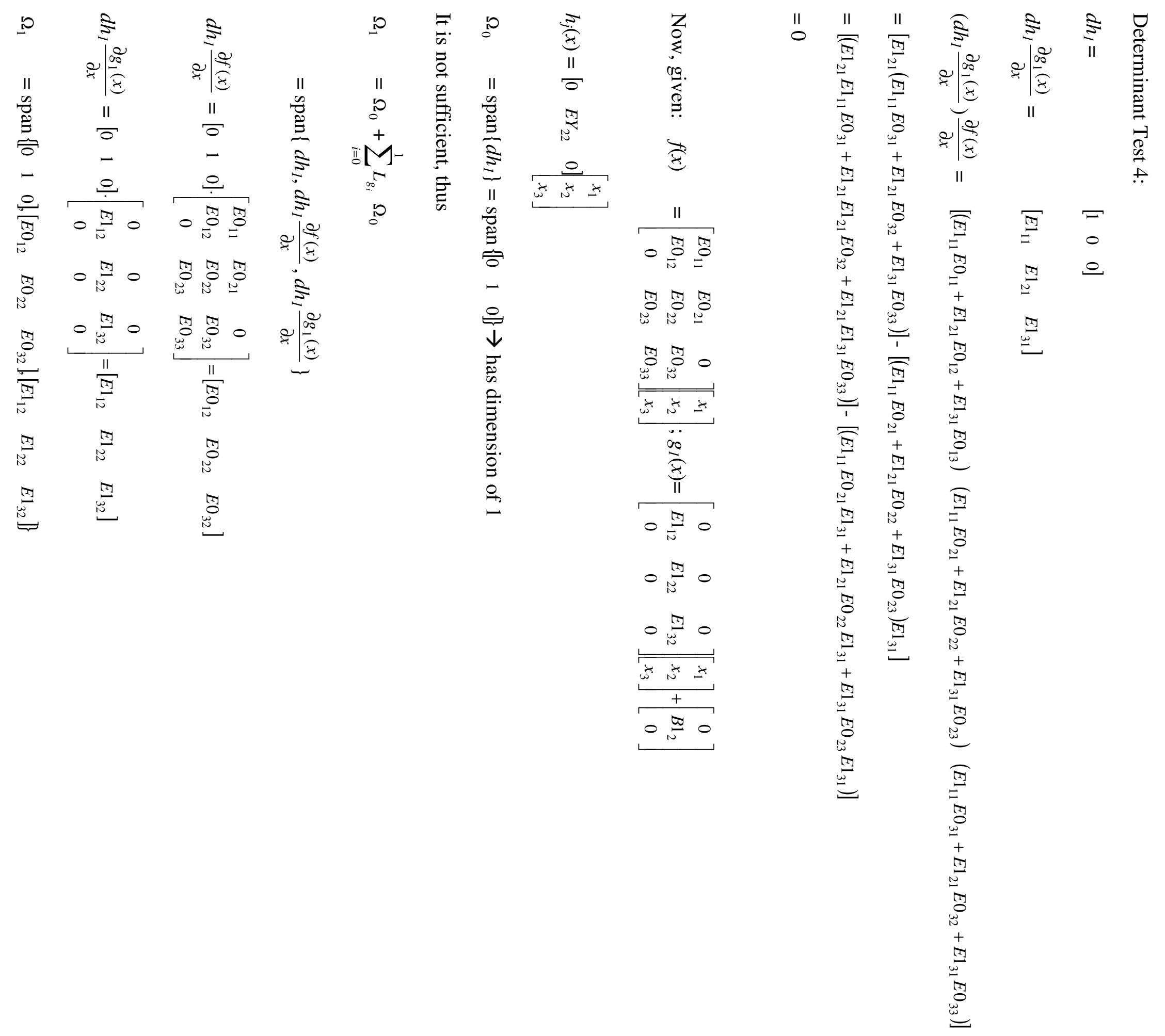




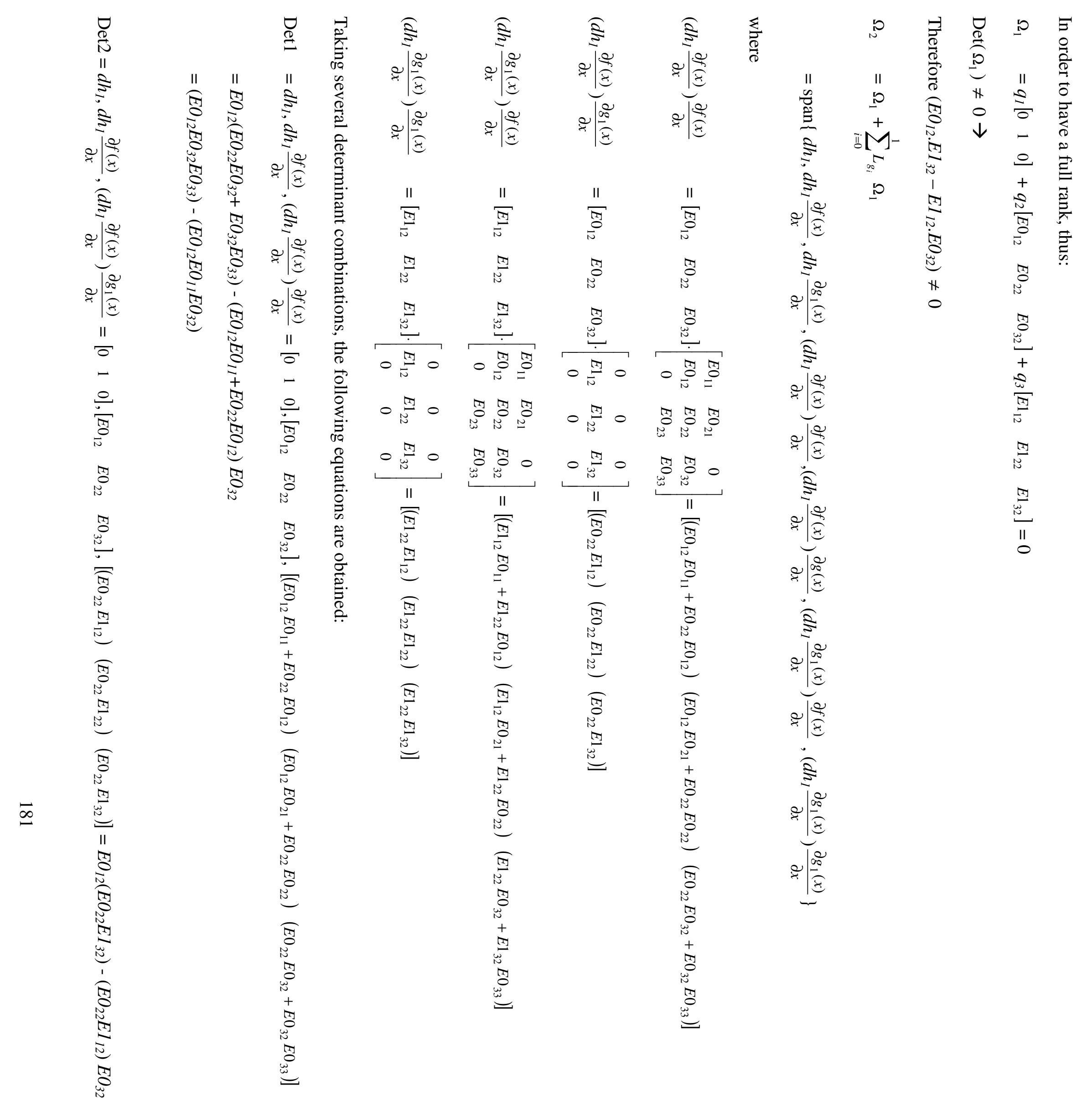




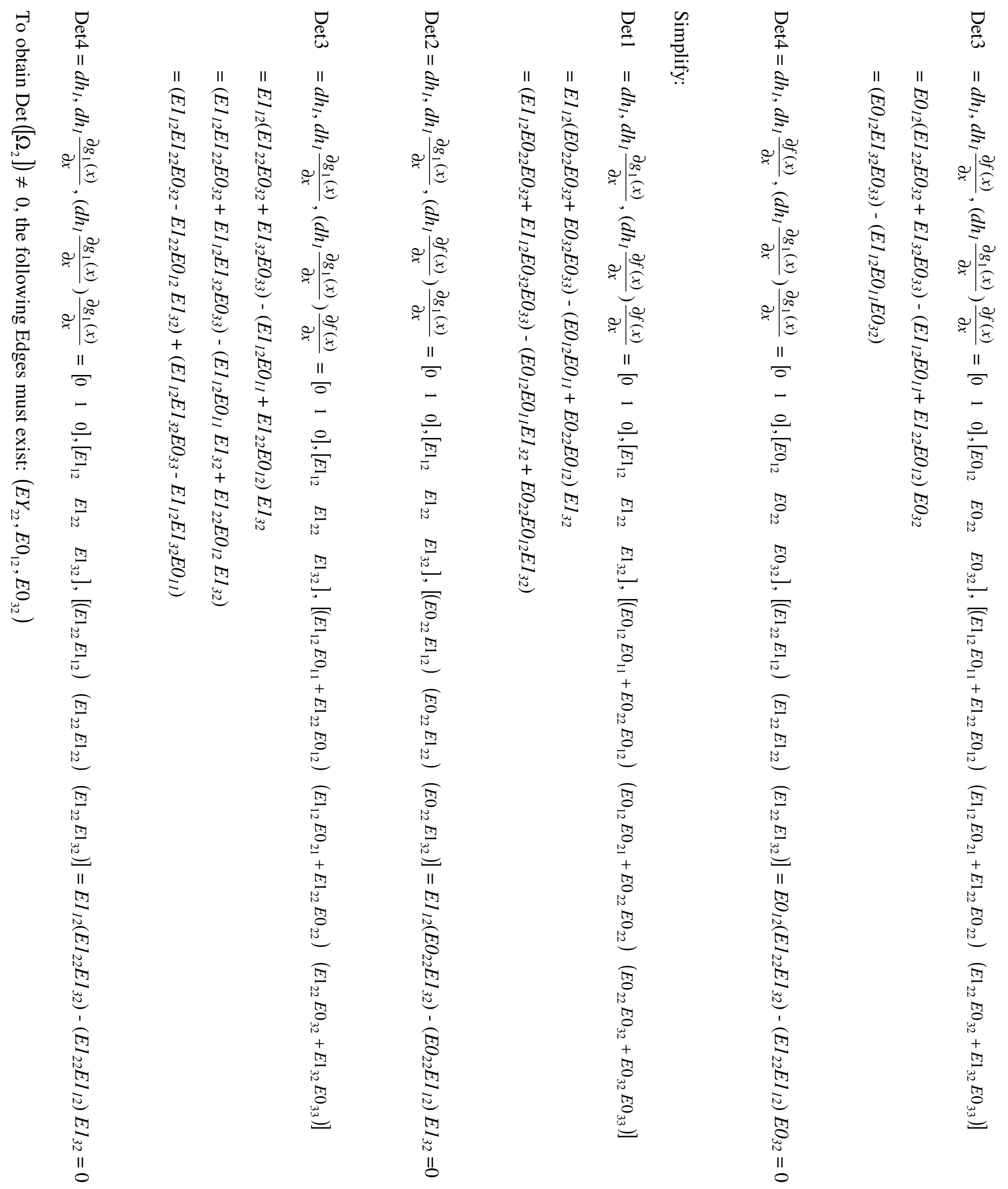




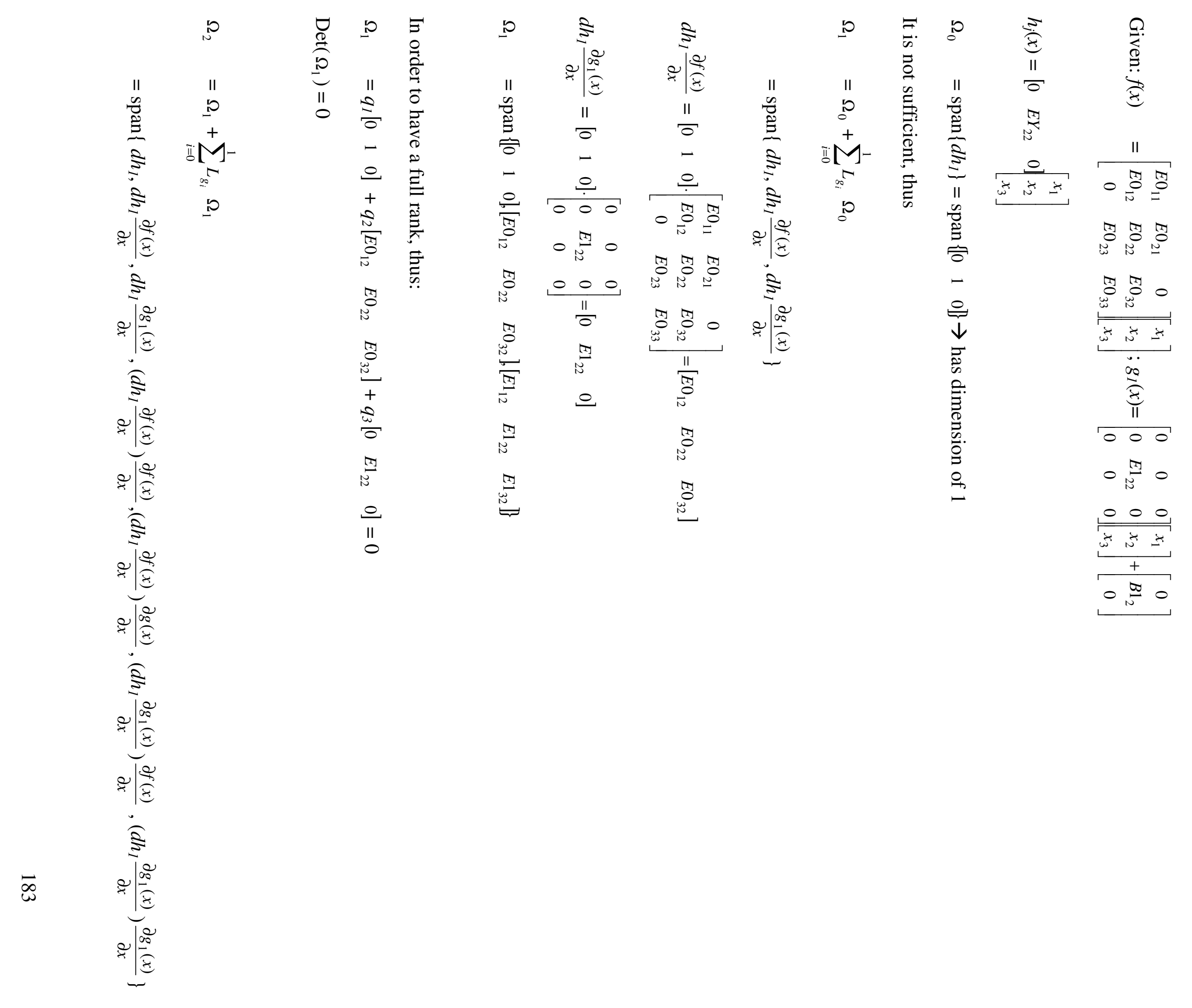




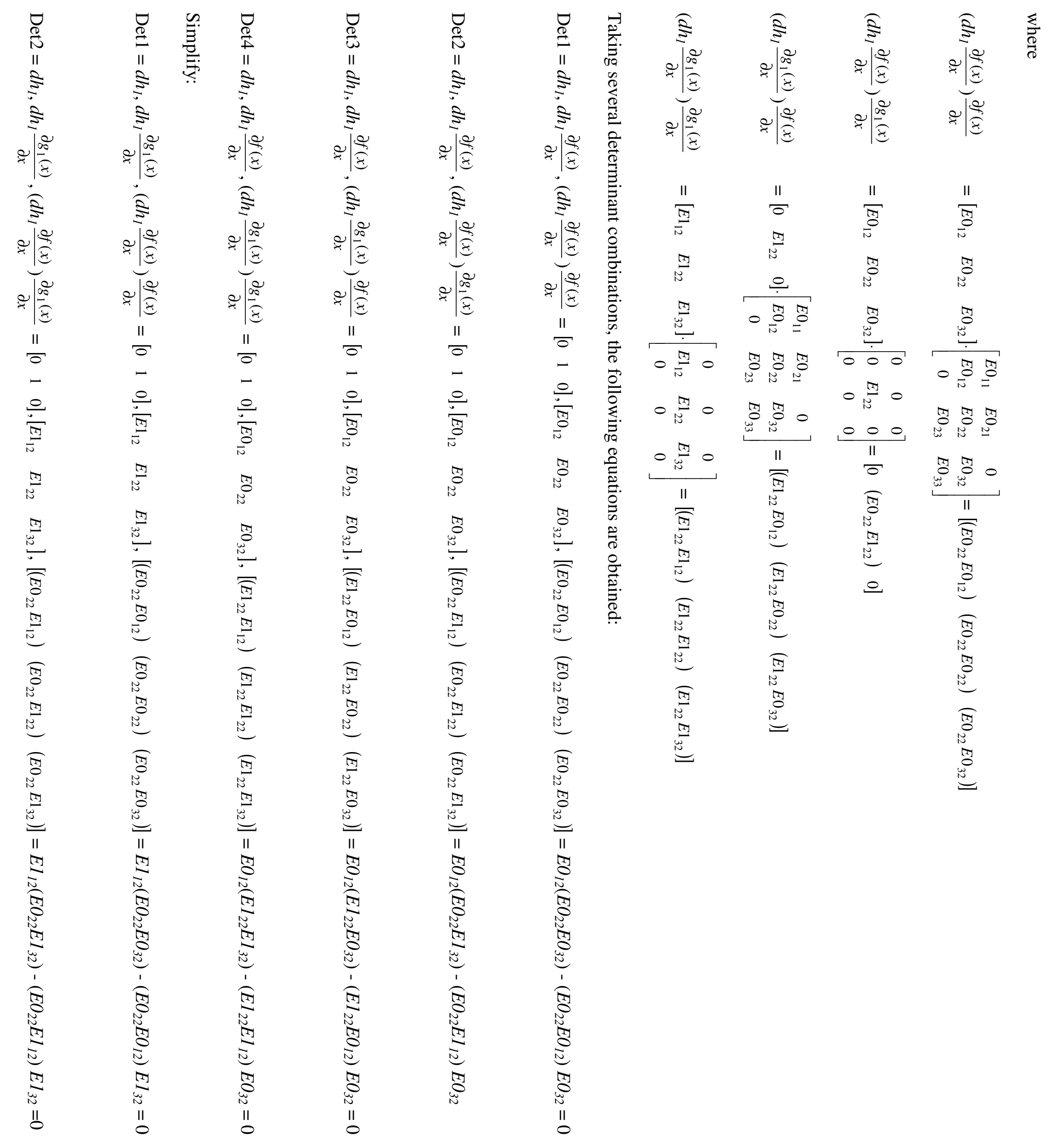




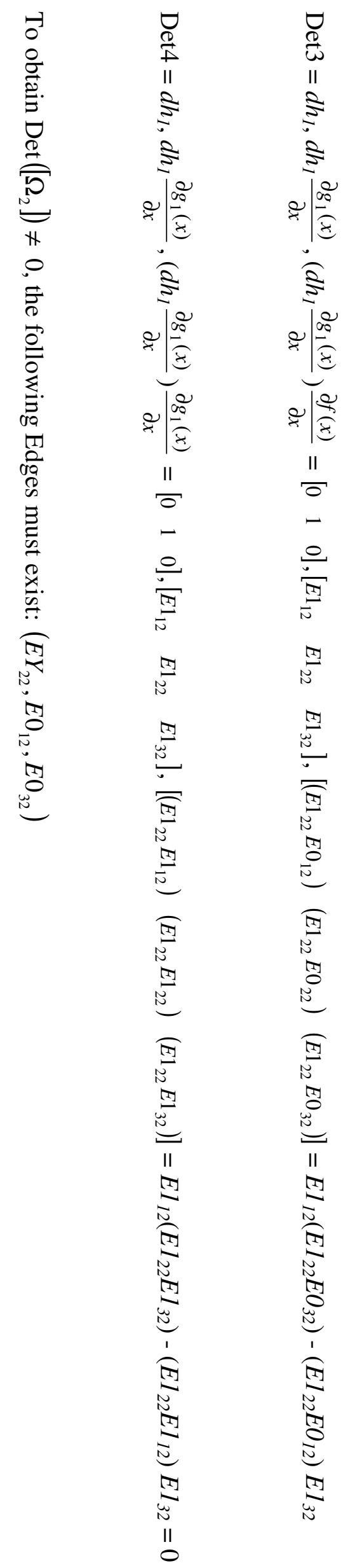

